\title{
E. Individuelle Gesundheitsleistungen von A - Z
}

\section{Allgemeine und fachübergreifende Leistungen}

\section{Spezielle Anamnesen - Körperliche und psychiatrische Untersuchungen Übersicht von A - Z}

Die nachfolgend aufgelisteten Leistungen sind die Grundlage für ggf. weitere zusätzliche apparative Untersuchungen (EKG, BelastungsEKG, Sono, Lungenfunktion, Röntgen, CT, NMW, Doppler, EEG, ENG, EMG) oder für die Behandlung.

Wichtig: Zur Ausstellung vieler Bescheinigungen sind vorher entsprechende körperliche Untersuchungen erforderlich.

\section{Spezielle Anamnesen im analogen Ansatz}

Abrechnung

\begin{tabular}{|l|l|l|}
\hline GOÄ Nr. & Kurzlegende & $2,3 f a c h €$ \\
\hline 30 & $\begin{array}{l}\text { Erhebung der homöopathischen Erstanamnese - mind. 1 Stunde } \\
\text { nach biographischen und homöopathisch-individuellen Gesichts- } \\
\text { punkten mit schriftlicher Aufzeichnung zur Einleitung einer homöopa- } \\
\text { thischen Behandlung }\end{array}$ & 120,65 \\
\hline $\begin{array}{l}\text { analog } \\
\text { analog ansetzbar für: } \\
\text { Erhebung der Erstanamnese vor Akupunktur } \\
\text { 1. Erhebung der Erstanamnese unter anthroposophischen Gesichts- } \\
\text { punkten } \\
\text { 2. Erhebung der Erstanamnese vor ayurvedischer Therapie } \\
\text { 3. Erhebung der Erstanamnese vor naturheilkundliche Therapie }\end{array}$ & 120,65 \\
\hline 31 & $\begin{array}{l}\text { Homöopathische Folgeanamnese - mind. 30 Minuten unter laufender } \\
\text { Behandlung nach den Regeln der Einzelmittelhomöopathie zur Beur- } \\
\text { teilung des Verlaufs und Feststellung des weiteren Vorgehens - ein- } \\
\text { schließlich schriftlicher Aufzeichnungen - }\end{array}$ & $\mathbf{6 0 , 3 3}$ \\
\hline $\begin{array}{l}\text { 31 } \\
\text { analog }\end{array}$ & $\begin{array}{l}\text { Analog ansetzbar für: } \\
\text { 1. Folgeanamnese bei Akupunktur } \\
\text { 2. Folgeanamnese bei anthroposophischer Therapie } \\
\text { 3. Folgeanamnese bei ayurvedischer Therapie } \\
\text { 4. Folgeanamnese bei naturheilkundlicher Therapie }\end{array}$ & $\mathbf{6 0 , 3 3}$ \\
\hline
\end{tabular}

Untersuchungen - Abrechnung

\begin{tabular}{|l|l|r|r|}
\hline GOÄ-Nr. & Kurzlegende & $\mathbf{1}$ fach $€$ & $\mathbf{2 , 3 f a c h} €$ \\
\hline $\mathbf{5}$ & Symptombezogene Untersuchung & 4,66 & $\mathbf{1 0 , 7 3}$ \\
\hline
\end{tabular}

Spezielle Untersuchungen

\begin{tabular}{|l|l|r|r|}
\hline $\mathbf{6}$ & Augen & 5,83 & $\mathbf{1 3 , 4 1}$ \\
\hline $\mathbf{7}$ & Bauchorgane & 9,33 & $\mathbf{2 1 , 4 5}$ \\
\hline $\mathbf{7}$ & Brustorgane & 9,33 & $\mathbf{2 1 , 4 5}$ \\
\hline $\mathbf{8}$ & Ganzkörperstatus & 15,15 & $\mathbf{3 4 , 8 6}$ \\
\hline $\mathbf{6}$ & Gefäßstatus & 5,83 & $\mathbf{1 3 , 4 1}$ \\
\hline $\mathbf{7}$ & Genitaltrakt - weiblicher & 9,33 & $\mathbf{2 1 , 4 5}$ \\
\hline $\mathbf{7}$ & Haut & 9,33 & $\mathbf{2 1 , 4 5}$ \\
\hline $\mathbf{6}$ & HNO-Bereich & 5,83 & $\mathbf{1 3 , 4 1}$ \\
\hline
\end{tabular}


E. Individuelle Gesundheitsleistungen von A - Z

\begin{tabular}{|l|l|r|r|}
\hline GOÄ-Nr. & Kurzlegende & $\mathbf{1 f a c h} \boldsymbol{\mathbf { 2 , 3 }}$ & $\mathbf{3 a c h} \mathbf{€}$ \\
\hline $\mathbf{3 2}$ & $\begin{array}{l}\text { Jugendarbeitsschutzuntersuchung (ggf. erforderliche weiter- } \\
\text { führende Diagnostik) }\end{array}$ & 23,32 & $\mathbf{5 3 , 6 2}$ \\
\hline $\begin{array}{l}\mathbf{3 2} \\
\text { analog }\end{array}$ & $\begin{array}{l}\text { Berufseignungsuntersuchung auf Patientenwunsch (ggf. erfor- } \\
\text { derliche weiterführende Diagnostik) }\end{array}$ & 23,31 & $\mathbf{5 3 , 6 2}$ \\
\hline $\mathbf{1 1}$ & Mastdarm und/oder Prostata - Digitaluntersuchung & 3,50 & $\mathbf{8 , 0 4}$ \\
\hline $\mathbf{8 0 0}$ & Neurologische Untersuchung & 11,37 & $\mathbf{2 6 , 1 4}$ \\
\hline $\mathbf{6}$ & $\begin{array}{l}\text { Nieren und harnableitende Wege, bei Männern zusätzl. Untersu- } \\
\text { chung Prostata, Prüfung Bruchpforten, Hoden und Nebenhoden }\end{array}$ & 5,83 & $\mathbf{1 3 , 4 1}$ \\
\hline $\mathbf{8 0 1}$ & Psychiatrische Untersuchung & 14,57 & $\mathbf{3 3 , 5 2}$ \\
\hline $\mathbf{6}$ & stomatognathes System & 5,83 & $\mathbf{1 3 , 4 1}$ \\
\hline $\mathbf{7}$ & Stütz- und Bewegungsorgane & 9,33 & $\mathbf{2 1 , 4 5}$ \\
\hline $\mathbf{1 0 0}$ & Untersuchung eines Toten - Ausstellung des Leichenscheines & 14,57 & $\mathbf{3 3 , 5 2}$ \\
\hline
\end{tabular}

\subsection{Früherkennung und Vorsorge}

Zahlreiche weitere Untersuchungen gibt es im Bereich der Früherkennung und Vorsorge. Für die Kinder-Früherkennungsuntersuchungen zwischen dem 14. und 18. Lebensjahr ist der analoge Ansatz der GOÄ Nr. 26 zu wählen.

\section{Abrechnung}

\begin{tabular}{|c|c|c|c|}
\hline GOÄ-Nr. & Kurzlegende & 1 fach $€$ & 2,3 fach $€$ \\
\hline 23 & Erste Vorsorgeuntersuchung in der Schwangerschaft & 17,49 & 40,22 \\
\hline 24 & Untersuchung im Schwangerschaftsverlauf & 11,66 & 26,81 \\
\hline 25 & Neugeborenen-Erstuntersuchung & 11,66 & 26,81 \\
\hline 26 & U2-Vorsorgeuntersuchung 3. - 10. Tag & 26,23 & 60,33 \\
\hline 26 & U3-Vorsorgeuntersuchung 4. -6. Lebenswoche & 26,23 & 60,33 \\
\hline 26 & U4-Vorsorgeuntersuchung 3. - 4. Lebensmonat & 26,23 & 60,33 \\
\hline 26 & U5-Vorsorgeuntersuchung 6. - 7. Lebensmonat & 26,23 & 60,33 \\
\hline 26 & U6-Vorsorgeuntersuchung 10. - 12. Lebensmonat & 26,23 & 60,33 \\
\hline 26 & U7-Vorsorgeuntersuchung 21. - 24. Lebensmonat & 26,23 & 60,33 \\
\hline 26 & U7A-Vorsorgeuntersuchung 3. Lebensjahr & 26,23 & 60,33 \\
\hline 26 & U8-Vorsorgeuntersuchung 43. - 48. Lebensmonat & 26,23 & 60,33 \\
\hline 26 & U9-Vorsorgeuntersuchung 60. - 64. Lebensmonat & 26,23 & 60,33 \\
\hline 26 & U10-Vorsorgeuntersuchung 7. - 8. Lebensjahr & 26,23 & 60,33 \\
\hline 26 & U11-Vorsorgeuntersuchung 9. - 10. Lebensjahr & 26,23 & 60,33 \\
\hline 26 & J1-Vorsorgeuntersuchung 13. - 14. Lebensjahr & 26,23 & 60,33 \\
\hline $\begin{array}{l}26 \\
\text { analog }\end{array}$ & J2-Vorsorgeuntersuchung 16. - 18. Lebensjahr - analog & 26,23 & 60,33 \\
\hline
\end{tabular}

\begin{tabular}{|l|l|r|r|}
\hline $\mathbf{2 7}$ & Krebsvorsorge bei einer Frau & 18,65 & $\mathbf{4 2 , 9 0}$ \\
\hline $\mathbf{2 8}$ & Krebsvorsorge bei einem Mann & 16,32 & $\mathbf{3 7 , 5 4}$ \\
\hline $\mathbf{2 9}$ & Gesundheitsuntersuchung eines Erwachsenen & 25,65 & $\mathbf{5 8 , 9 9}$ \\
\hline
\end{tabular}

Neben den Nrn. 23-29 können die Nrn. 1-8 nicht abgerechnet werden. 


\section{Beratungen und Erörterungen}

Bei fast allen ärztlichen Leistungen zur Diagnostik und Therapie wie auch bei IGEL-Leistungen sind Beratungen und Untersuchungen - vorher oder nachher - erforderlich. In der Regel werden erst danach weitere Leistungen zur Diagnostik und/oder Therapie eingesetzt, die teilweise im Rahmen gestatteter kurativer Behandlung zu Lasten der GKV oder PKV erbracht und abgerechnet werden können, aber natürlich auch gemäß einer Vereinbarung mit dem Patienten als IGEL-Leistung.

Im Beratungsbereich stehen eine einfache Beratung (GOÄ Nr. 1), eine ausführliche Beratung, mind. 10 Min. (GOÄ Nr. 3) und eine Erörterung, mind. 20 Min. (GOÄ Nr. 34) zur Verfügung und werden auch in unseren Abrechnungshinweisen immer wieder nebeneinander angegeben. Welche der Leistungen anzusetzen ist, muss der beratende Arzt entscheiden!

Die nachfolgend beispielhaft aufgelisteten Beratungsleistungen sind ggf. um zusätzliche körperliche und apparative Untersuchungen zu erweitern - mit Ausnahme der GOÄ Nr. 3, die nur alleine oder neben den GOÄ Nrn. 5-8, 800 oder 801 im Rahmen desselben Arzt- und Patientenkontaktes angesetzt werden darf: Nr. 3 nie neben Sonderleistungen!

\subsection{Beratungen}

Beratungen, die z. B. abhängig von der Beratungsintensität und Dauer nach den GOÄ Nrn. 1 oder 3 abgerechnet werden können:

- Beratung vor einer Anti-Aging-Behandlung

- Beratung vor einer gewünschten Arzneimittel-Behandlung, z. B. „Life-Style“-Medikation zur Potenzförderung, zur Gewichtsreduktion, gegen androgenetischen Haarausfall, Laxantien, Medikamente zur Stressprophylaxe

- Ernährungs-/Diätberatung

- Beratung zur Zusammenstellung und Anwendung einer Haus- oder Reiseapotheke

- Spezielle Impfberatung (Impfungen außerhalb der GKV)

- Beratung vor kosmetischen Behandlungen oder Eingriffen

- Beratung im Zusammenhang mit der Veranlassung von Laboruntersuchungen auf Wunsch des Patienten - z. B. PSA Bestimmung

- Medizinisch-kosmetische Beratung

- Reisemedizinische Beratung

- Sonnenlicht- und Hauttypberatung

- Sportmedizinische Beratung

- Umweltmedizinisch orientierende Beratung, z. B. bezogen auf berufliche oder private Kontakte mit umweltschädlichen Stoffen

- Beratung zur Ausweitung von Vorsorgeuntersuchungen

\section{Abrechnung}

\begin{tabular}{|l|l|r|r|}
\hline GOÄ Nr. & Kurzlegende & $\mathbf{1 f a c h} €$ & $\mathbf{2 , 3 f a c h} €$ \\
\hline $\mathbf{1}$ & Beratung & 4,66 & $\mathbf{1 0 , 7 3}$ \\
\hline $\mathbf{3}$ & Eingehende Beratung (10 Min.) nicht neben Sonderleistungen & 8,74 & $\mathbf{2 0 , 1 1}$ \\
\hline $\mathbf{2 1}$ & Humangenetische Beratung & 20,98 & $\mathbf{4 8 , 2 6}$ \\
\hline $\mathbf{2 2}$ & Beratung einer Schwangeren im Konfliktfall & 17,49 & $\mathbf{4 0 , 2 2}$ \\
\hline $\mathbf{8 1 7}$ & $\begin{array}{l}\text { Eingehende psychiatrische Beratung einer Bezugsperson psy- } \\
\text { chisch gestörter Kinder oder Jugendlicher }\end{array}$ & 10,49 & $\mathbf{2 4 , 1 3}$ \\
\hline
\end{tabular}

\section{Kommentar:}

Nach Brück betrifft der Ausschluss aller Sonderleistungen - ausgenommen GOÄ Nrn. 5, 6, 7 und 8 - neben GOÄ Nr. 3 nicht die GOÄ Nrn. 70 und 75, da deren Leistung zu einem Zeitpunkt erfüllt wird, der nicht im zeitlichen Zusammenhang mit der Leistungserbringung nach GOÄ Nr. 3 steht. 
E. Individuelle Gesundheitsleistungen von A - Z

Abrechnung

Auf einen Blick: Beratungen rund um die Uhr

\begin{tabular}{|c|c|c|c|}
\hline GOÄ Nr. & Kurzlegende & 1 fach $€$ & $1-/ 2,3$ fach $\left.€^{1}\right)$ \\
\hline \multicolumn{4}{|c|}{\begin{tabular}{|l|} 
Beratung - auch telefonisch \\
\end{tabular}} \\
\hline $\left.1+A^{1}\right)$ & außerhalb der Sprechstunde & $4,66+4,08$ & $10,73+4,08$ \\
\hline $1+B+D$ & telefonische am Samstag 20.30 Uhr & $4,66+10,49+12,82$ & $10,73+10,49+12,82$ \\
\hline $1+B+D$ & $\begin{array}{l}\text { nachts 20-22 Uhr, 6-8 Uhr am Wochen- } \\
\text { ende oder Feiertag }\end{array}$ & $4,66+10,49+12,82$ & $10,73+10,49+12,82$ \\
\hline $1+B$ & in der Nacht 20-22 Uhr, 6-8 Uhr: & $4,66+10,49$ & $10,73+10,49$ \\
\hline $1+C$ & in tiefer Nacht 22-6 Uhr & $4,66+18,65$ & $10,73+18,65$ \\
\hline $1+C+D$ & $\begin{array}{l}\text { in tiefer Nacht 22-6 Uhr am Wochenen- } \\
\text { de o. Feiertag }\end{array}$ & $4,66+18,65+12,82$ & $10,73+18,65+12,82$ \\
\hline $1+D$ & tagsüber am Wochenende & $4,66+12,82$ & $10,93+12,82$ \\
\hline
\end{tabular}

\begin{tabular}{|c|c|c|c|}
\hline \multicolumn{4}{|c|}{ Eingehende Beratungen - auch telefonisch ${ }^{2}$ ) } \\
\hline $3+\mathrm{A}$ & außerhalb der Sprechstunde & $8,74+4,08$ & $20,11+4,08$ \\
\hline $3+B+D$ & am Samstag 20.30 & $8,74+10,49+12,82$ & $\begin{array}{l}20,11+10,49+ \\
12,82\end{array}$ \\
\hline $3+B$ & in der Nacht 20-22 Uhr, 6-8 Uhr & $8,74+10,49$ & $20,11+10,49$ \\
\hline $3+B+D$ & $\begin{array}{l}\text { nachts 20-22 Uhr, 6-8 Uhr am Wochen- } \\
\text { ende oder Feiertag }\end{array}$ & $8,74+10,49+12,82$ & $\begin{array}{l}20,11+10,49+ \\
12,82\end{array}$ \\
\hline $3+\mathrm{C}$ & in tiefer Nacht 22- $6 \mathrm{Uhr}$ & $8,74+18,65$ & $20,11+18,65$ \\
\hline $3+C+D$ & $\begin{array}{l}\text { in tiefer Nacht 22-6 Uhr am Wochenen- } \\
\text { de o. Feiertag }\end{array}$ & $8,74+18,65+12,82$ & $20,11+18,65+12,82$ \\
\hline $3+D$ & am Wochenende & $8,74+12,82$ & $20,11+12,82$ \\
\hline
\end{tabular}

\section{Beratungen in regelmäßiger Samstagssprechstunde}

\begin{tabular}{|l|l|l|l|}
\hline $\mathbf{1 + 1 / 2} \mathbf{D}$ & $\begin{array}{l}\text { Beratung in regelmäßiger Samstags- } \\
\text { sprechstunde }\end{array}$ & $4,66+6,41$ & $\mathbf{1 0 , 7 3 + 6 , 4 1}$ \\
\hline $\mathbf{3 + 1 / 2} \mathbf{D}$ & $\begin{array}{l}\text { Eingehende Beratung in regelmäßiger } \\
\text { Samstagsprechstunde }\end{array}$ & $8,74+6,41$ & $\mathbf{2 0 , 1 1 + 6 , 4 1}$ \\
\hline
\end{tabular}

1) Alle Zuschläge (A, B, C, D) dürfen nach GOÄ nur mit dem 1-fachen Satz berechnet werden

2) Nr. 3 nicht neben Sonderleistungen

\subsection{Erörterungen}

Erörterungen, die z. B. nach GOÄ Nr. 34 (analog) abgerechnet werden können:

- Erörterung einer Anti-Aging-Behandlung, z. B. Risiken einer Hormontherapie

- Erörterung einer gewünschten Arzneimittel-Behandlung, z. B. „Life-Style Medikamente“ zur Potenzförderung, zur Gewichtsreduktion, gegen Glatzenbildung

- Erörterung einer gesunden Ernährung/Diät

- Adipositasberatung (Auswirkung auf die Lebensführung)

- Ausgedehnte Beratung zur Zusammenstellung und Anwendung einer Haus- oder Reiseapotheke

- Begleitende Beratung und Betreuung bei Verordnung von Lifestyle-Arzneimitteln außerhalb der GKV-Leistungspflicht

- Erörterung vor kosmetischen Behandlungen oder Eingriffen

- Erörterung der Ergebnisse im Zusammenhang mit der Veranlassung von Laboruntersuchungen auf Wunsch des Patienten - Laborleistungen s. Seite 229.

- Raucherentwöhnung

- Second Opinion: Erörterung von diagnostischen und therapeutischen Maßnahmen, z. B. Operationen, Bestrahlungen, S. Seite 102 
- Erörterung von Selbstmedikation im Rahmen von Prävention und Lebensführung

- Erörterung über sportliche Betätigungen

- Umweltmedizinisch orientierende Erörterung z. B. bezogen auf berufliche oder private Kontakte mit umweltschädlichen Stoffen.

- Beratung zur Selbstmedikation im Rahmen von Prävention und Lebensführung

Erörterungen sind in vielen Fällen vor und nach einer Diagnostik und/oder Behandlung erforderlich.

\begin{tabular}{|l|l|r|r|}
\hline GOÄ Nr. & Kurzlegende & $\mathbf{1 f a c h} €$ & $\mathbf{2 , 3 f a c h} €$ \\
\hline $\mathbf{3 4}$ & $\begin{array}{l}\text { Erörterung, mind. 20 Min. - häufig als analoger Ansatz ent- } \\
\text { sprechend § 6 (2) GOÄ }\end{array}$ & 17,49 & $\mathbf{4 0 , 2 2}$ \\
\hline
\end{tabular}

\section{Second Opinion}

In den USA ist es - wenn die eigenen Finanzen es zulassen - üblich, bei lebensverändernden Diagnosen oder vor großen Operationen eine zweite ärztliche Meinung - Second Opinion - einzuholen. In Österreich und der Schweiz werden die Krankenversicherten angehalten, bei bestimmten Erkrankungen eine Second Opinion einzuholen. In der Schweiz erhält der Versicherte dafür sogar einen Rabatt.

Bei einer Second Opinion geht es dem Patienten darum, eine gestellte Diagnose und/oder eine vorgeschlagene Therapie zu hinterfragen und neu mit einem anderen Mediziner zu diskutieren. Dabei wird - da in der Regel die erhobenen Befunde mitgebracht werden können - nur in wenigen Fällen eine neue oder weiterführende Diagnostik notwendig sein. Auch in der Bundesrepublik nehmen in diesem Kontext Nachfrage und Angebot zu.

Im Internet www.tumorbio.uni-freiburg. de/03 beraten/03 01.html bietet die Klinik für Tumorbiologie in Freiburg "Second Opinion - die zweite Meinung über die Erkrankung und deren Therapiemöglichkeiten."an.

Die Klinik informiert: ... „Mit dem Angebot „Second Opinion“ wollen wir die Kompetenz der Patienten im Umgang mit der eigenen Erkrankungssituation stärken... Die Second Opinion ist für Patienten gedacht, die sich eingehend mit ihrer eigenen Erkrankungssituation auseinandersetzen möchten, eine Informations- und Orientierungshilfe suchen und neben einer fachonkologischen Begutachtung auch zu einem ganzheitlich ausgerichteten Strategieplan beraten werden wollen.

Anhand der vorhandenen Krankheitsunterlagen (Befundberichte, Röntgenbilder, Kernspin/CT-Aufnahmen, u. a.) und einer aktuellen klinischen Untersuchung erfolgt eine Bewertung der Erkrankungs- und Behandlungssituation sowie die Ableitung von Empfehlungen des weiteren Vorgehens. Durch die Einbindung weiterer Fachberater aus den Bereichen Psycho-onkologie, Ernährung, Unkonventionelle Therapien, Physiotherapie und Pflege werden die wichtigsten Aspekte im Zusammenhang mit der Tumorerkrankung erörtert. Ziel der Beratung ist die Stärkung der Patientenkompetenz durch:

- Unterstützung im Entscheidungsprozess bei komplexen Fragen der Diagnostik und Behandlung,

- eine Orientierungshilfe angesichts der Informationsfülle, die sich durch die bis dahin erfolgten Beratungen, Berichte ergeben und aus weiteren Quellen (Bücher, Fernsehen, Internet, u. a.) gewonnen wurden,

- psychosoziale Beratung zur Krankheitsverarbeitung und Bewältigung,

- eine Stärkung des Selbsthilfepotentials..."

AOK Duo - Die ärztliche Zweitmeinung

Für AOK-Versicherte bietet die AOK- Bremen/Bremerhaven unter http://www.aok.de/bremen/aokduo-die-aerztliche-zweitmeinung-160246.php einen Dienst an. Die AOK informiert:

... „Versicherte können bei schweren Erkrankungen kostenlos eine ärztliche Zweitmeinung einholen. Die AOK kümmert sich um alles.

Tagtäglich erfahren Menschen, dass sie schwer krank sind: „Sie haben Krebs“ oder „Wir müssen Sie am Herzen operieren" müssen sie von ihrem Arzt hören. Doch wie können sie mit dieser schockierenden Diagnose umgehen - was können sie jetzt tun? Die AOK Bremen/Bremerhaven bietet ihren Versicherten seit 2010 die Möglichkeit, sich eine zweite ärztliche Meinung von einem ausgewiesenen Experten zu holen. Das soll den Patienten helfen, die Krankheit zu akzeptieren, damit besser umzugehen und die weitere Behandlung zu planen. 
E. Individuelle Gesundheitsleistungen von A - Z

\section{Im Mittelpunkt: das persönliche Gespräch}

Derzeit bieten 17 Ärztinnen und Ärzte aus Bremen und Bremerhaven ihr Fachwissen im Rahmen von „AOK Duo: Die ärztliche Zweitmeinung“ an. Die meisten von ihnen sind als erfahrene Chefärzte, leitende Oberärzte oder niedergelassene Mediziner tätig. Das persönliche Gespräch steht im Mittelpunkt von AOK Duo: Der Spezialist soll den Patienten hier noch einmal die medizinischen Zusammenhänge erläutern, Fragen beantworten und sich Zeit für die Versicherten nehmen - es sollen keine Fragen offen bleiben. Das gilt selbstverständlich auch bei der Erkrankung von Kindern. Das wiederum gibt Patienten und Angehörigen Sicherheit und Vertrauen in die ärztlichen Maßnahmen. Auf Wunsch des Patienten bespricht der Spezialist die weitere Therapie auch mit dem behandelnden Arzt.

\section{Vermittlung von kurzfristigen Terminen}

Die AOK Bremen/Bremerhaven vermittelt im Rahmen von „AOK Duo: Die ärztliche Zweitmeinung“ bei folgenden lebensverändernden Diagnosen kurzfristig Termine bei den Experten:

- Angeborene Fehlbildung bei Kindern

- Schwere Herz-Kreislauferkrankungen mit OP-Indikation

- Krebserkrankungen bei Kindern und Erwachsenen

- Asthma bronchiale bei Kindern

- Schwere Nierenerkrankungen bei notwendiger Dialyse

- Bandscheibenerkrankungen mit OP-Indikation

- Gehirntumore

Mit der Zweitmeinung soll die Arbeit des erstbehandelnden Arztes ausdrücklich nicht geschmälert werden, die Ursprungsbefunde sind vielmehr Grundlage der ärztlichen Zweitmeinung. Eine langwierige Suche der Patienten nach einem auf diese Krankheit spezialisierten Mediziner entfällt, ebenso wie das Problem, bei diesem Arzt einen zeitnahen Termin zu bekommen. Mit AOK Duo erhalten die AOK-Versicherten einen Termin in der Regel innerhalb von acht Tagen..."

Auch die AOK Nordwest (http://www.aok.de/nordwest/index.php ) vermittelt ärztliche Zweitmeinung bei schwerwiegenden Erkrankungen

Im Oktober 2012 wurde im Deutschen Ärzteblatt bekannt gegeben:

..."Mit der ärztlichen Zweitmeinung möchten wir unseren Versicherten schwierige Entscheidungen erleichtern und mehr Sicherheit geben, um mit Vertrauen und Zuversicht die nächsten Behandlungsschritte anzugehen“, sagte Martin Litsch, Vorstandsvorsitzender der AOK Nordwest. Versicherte, die das Angebot nutzen möchten, sollen sich dafür an ihr AOK-Kundencenter wenden.

Die KV Schleswig-Holstein begrüßt die AOK-Initiative. „Gerade in einem sensiblen Bereich wie der Krebsbehandlung kann das Vier-Augen-Prinzip unter Hinzuziehung eines weiteren Experten von Vorteil sein“, sagte deren Vorstandsvorsitzende Monika Schliffke.

Zustimmung zu dem neuen Angebot kam auch von der Hochschulmedizin. „Die Spezialisten des Universitätsklinikums Schleswig-Holstein begrüßen die Initiative der AOK, denn hochkomplexe Diagnosen bedürfen einer sorgfältigen Abklärung auf dem neuesten Stand der Forschung", sagte der Vorstandsvorsitzende des Universitätsklinikums Schleswig-Holstein, Jens Scholz. Die Universitätsklinik beteilige sich an den beiden Standorten Lübeck und Kiel an dem AOK-Projekt. ..."

\section{Recht auf Zweitmeinung}

lautet der Name eines Portals der Techniker Krankenkasse (TK) http://www.tk.de/tk/behandlungen/ zweitmeinung/recht-auf-zweitmeinung/213558. DleKasse informiert:

... ,Jeder Versicherte hat das Recht, bei Zweifeln an der vorgeschlagenen Therapie einen anderen Arzt aufzusuchen, um sich eine zweite Meinung einzuholen.

Dies ist besonders sinnvoll etwa bei schwerwiegenden Krankheiten, langfristigen Behandlungen oder auch bei planbaren operativen Eingriffen. Hier hilft eine zweite ärztliche Meinung dem Patienten, die Chancen und Risiken der vorgeschlagenen Therapie besser einzuschätzen. Bei Operationen können beispielsweise Nerven und Gefäße geschädigt werden oder Narben und Verwachsungen auftreten. Bei einer medikamentösen Therapie können unerwünschte Nebenwirkungen auftreten, bei einer manuellen Therapie sind Verletzungen möglich.

Um die Entscheidung für eine Therapie zu überprüfen, muss nicht das gesamte diagnostische Verfahren von vorne beginnen. Der Patient hat das Recht, seine gesamten medizinischen Unterlagen wie etwa Untersuchungsbefunde oder Röntgenbilder einzusehen. Die Praxis kann gegen eine Gebühr gegebenenfalls Kopien zur Verfügung stellen. 
Darüber hinaus haben TK-Versicherte die Möglichkeit, sich bei Zweifeln oder Fragen zur Therapie ihres behandelnden Arztes an das TK-Zweitmeinungs-Telefon zu wenden. ..."

- Vorsicht Operation (http://www.vorsicht-operation.de/) und Medexo (https://www.medexo.com/) sind Portale zur Erlangung von Zweitmeinungen.

Vorsicht Operation ist ein Service der Medexo GmbH - Medizinische Experten Online.

Medexo schreibt u. a. über sich:

... „Der Name Medexo steht für Medizinische Experten Online.

Medexo bietet Ihnen als Patienten die Möglichkeit, eine medizinische Zweitmeinung von führenden und unabhängigen Spezialisten über das Internet einzuholen. Speziell für diesen Zweck entwickelte Fragebögen, sowie ein einzigartiges Programm zur Übertragung von medizinischen Unterlagen, ermöglichen die Erfassung der für die Zweitmeinung erforderlichen Informationen.

Für jede Disziplin steht ein Ärztebeirat zur Verfügung. Dieser Beirat an renommierten aktiven und inaktiven Spezialisten des jeweiligen Fachbereiches organisiert und kontrolliert die Prüfung unserer teilnehmenden Experten.

Medexo ist eine Weiterentwicklung des aus der Presse bekannten Portals „Vorsicht!Operation“. Zusammen mit den Spezialisten, die für die Zweitmeinungen zur Verfügung stehen, wird Medexo von einem Team aus Medizinern und Internetexperten betreut und ständig auf dem neuesten Stand der Wissenschaft gehalten. Unser Ziel ist es, Ihnen in naher Zukunft Zweitmeinungen für alle medizinischen Fachgebiete anbieten zu können..."

\section{Im Internet sind folgende Adressen hilfreich:}

www.leitlinien.de/

äzq: Zentralstelle der Deutschen Ärzteschaft zur Qualitätssicherung in der Medizin, GbR. Gemeinsame Einrichtung der Bundesärztekammer und der Kassenärztlichen Bundesvereinigung

Hier finden Sie Antworten auf häufig gestellte Fragen, Zugang zu Leitlinien-Datenbanken und -Listen aus dem In- und Ausland (z. B. AWMF, NGC, SIGN) und Leitlinien verschiedener Anbieter für ausgewählte Krankheitsbilder (z. B. Kopfschmerzen).

www.uni-duesseldorf.de/WWW/AWMF/I/II_list.htm

AWMF: Arbeitsgemeinschaft der Wissenschaftlichen Medizinischen Fachgesellschaften

Es handelt sich um wissenschaftlich begründete Leitlinien für Diagnostik und Therapie, die von Wissenschaftlichen Medizinischen Fachgesellschaften erarbeitet wurden. Zahlreiche dieser Leitlinien erscheinen unter mehreren Rubriken. Die Leitlinien sind in einer systematischen Ordnung nach medizinischen Fachgebieten aufgelistet

www.rki.de/

\section{Robert Koch Institut:}

Die Aufträge des Robert Koch-Instituts sind

- Beobachtung des Auftretens von Krankheiten und Risikofaktoren in der Bevölkerung

- Gewährleistung wissenschaftlicher Untersuchungen.

Damit soll ermöglicht werden, die erforderlichen Maßnahmen zum Schutze der Gesundheit der Bevölkerung zu treffen. Das RKI ist die zentrale Einrichtung und das Koordinationszentrum des Bundesministeriums für Gesundheit und Soziales (BMGS) für angewandte- und Grundlagenforschung auf den Gebieten der

- Infektionskrankheiten, inklusive Infektionsepidemiologie; z. B. finden sich hier wichtige Informationen zur ansteckende Atemwegserkrankung - Schweres Akutes-Respiratorisches Syndrom = SARS.

- Epidemiologie nicht übertragbarer Krankheiten.

... „Auf dieser Grundlage ist das RKI in der Lage, Maßnahmen vorzuschlagen, die sich auf Intervention und Prävention, auf die Beseitigung von Erkenntnisdefiziten und auf die Qualitätssicherung der Gesundheitsvorsorge und -versorgung beziehen. Es ist an der Erarbeitung von Richtlinien, Empfehlungen, Gutachten für Bundesregierung, Parlament, Fachöffentlichkeit und übrige Gesundheitspolitik beteiligt..." (Quelle: Text auf Internetseite des RKI)

www.dimdi.de/de/dimdi/index.htm

\section{DIMDI: Deutsches Institut für Medizinische Dokumentation und Information}

DIMDI bietet für die Schwerpunkte Gesundheitswesen, Medizin, Pharmakologie, Toxikologie und Biologie ein umfassendes Spektrum von Datenbanken (teilweise kostenpflichtig) aus dem gesamten Bereich der biowissenschaftlichen Disziplinen an. 


\section{E. Individuelle Gesundheitsleistungen von A - Z}

Zahlreiche Empfehlungen der Fachgesellschaften zu Diagnostik und Therapie, die nicht den Charakter einer Leitlinie haben, finden sich nur direkt in den Internetauftritten der Fachgesellschaften z. B. unter

www.dgppn.de

Die Deutschen Gesellschaft für Psychiatrie, Psychotherapie und Nervenheilkunde (DGPPN) bietet Kurzfassungen einzelner Leitlinien zum download im PDF-Format z. B.: Behandlungsleitlinie Schizophrenie, Leitlinien zu Diagnostik und Therapie von Angsterkrankungen, Behandlungsleitlinie Demenz, Behandlungsleitlinie Ess-Störungen usw. - Besonders wird aber auf die ungekürzte Veröffentlichung dieser Leitlinien im Buchform hingewiesen.

www.dgn.org/

Die Gesellschaft für Neurologie bietet zahlreiche Leitlinien an, von denen die meisten auch auf der AWMF-Seite zu finden sind.

Wer also zu einem Thema fündig werden will, muss die entsprechenden Fachgesellschaften absurfen. Der niedergelassene Allgemeinmediziner/Facharzt in der Nähe des Patienten wird auf Dauer die besten Chancen haben, ein gefragter Berater für die „Zweite Meinung“ zu sein, wenn er Kollegen der entsprechenden Fachgebiete aus Klinik und Praxis mit einbezieht und sich ein Berater-Team bildet. Ohne gute Kontakte zu einem oder mehreren routinierten leitenden Klinikern eines großen Krankenhauses wird die Second Opinion nie hochqualifiziert sein können.

Jede größere Operation, Strahlentherapie oder Zytostatika-Therapie bringt für Patienten in unterschiedlicher Weise „Angst“ mit sich, die dadurch etwas gemildert werden kann, dass ein 2. med. Experte berät.

Besonders angenehm wird es der Patient empfinden, wenn sich beide Behandlungsvorschläge decken, d. h. wenn der 2. Experte eine ähnliche Meinung vertritt wie der Arzt, der die primäre Diagnose gestellt hat.

\section{Die Frage der Abrechnung einer Second Opionin ist einfach zu beantworten:}

1. Kommt der Patient persönlich zum beratenden Arzt, sind die entsprechenden Beratungs- und ggf. Untersuchungsziffern der GOÄ ansetzbar.

2. Wird ohne persönlichen Kontakt und nur über den telefonischen Weg eine Beratung gewünscht, so ist diese nach den bekannten GOÄ-Beratungsziffern abrechenbar. In der Regel erhält der Arzt aber bestehende Befunde zugeschickt und äußert sich schriftlich dazu (Erstellung eines Gutachtens nach Aktenlage = GOÄ Nr. 85). Ist das Beratungsgutachten sehr umfangreich, kann der Arzt einen höheren Multiplikator wählen.

Wir raten dringend, von dem Patienten zur Vermeidung etwaiger Auseinandersetzungen über Art der Erstellung und der Rechnungslegung eine schriftliche Erklärung, z. B. nachfolgenden Inhaltes unterschreiben zu lassen:

\section{Einverständniserklärung:}

Ich, ................................, wünsche von ............................... eine Zweitmeinung (Second Opinion) zu Therapie und/oder Diagnostik folgender Erkrankung:

Ich bin damit einverstanden, dass diese Zweitmeinung auf der Basis der von mir zur Verfügung gestellten schriftlichen Befunde und gegebenenfalls weiterer schriftlicher Unterlagen schriftlich erstellt und mir übermittelt wird. Ein unmittelbarer persönlicher Arzt-Patienten-Kontakt findet nicht statt.

Die Rechnung erfolgt nach den Bestimmungen der Gebührenordnung für Ärzte (GOÄ)

\section{Wichtig:}

Ist der Patient bei einer gesetzlichen Krankenkasse versichert und nimmt der die Second Opinion erbringende Arzt an der vertragsärztlichen Versorgung teil, bitte unbedingt auch an die schriftliche Zustimmung zur Privatvergütung nach den Bestimmungen des Bundesmantelvertrages denken! 
Abrechnung nach Befunden

\begin{tabular}{|l|l|r|r|}
\hline GOÄ Nr. & Kurzlegende & $\mathbf{1 f a c h} €$ & $\mathbf{2 , 3 f a c h} €$ \\
\hline $\mathbf{3}$ & $\begin{array}{l}\text { Eingehende Beratung (mind.10 Min.) -nicht neben Sonderleis- } \\
\text { tungen }\end{array}$ & 8,74 & $\mathbf{2 0 , 1 1}$ \\
\hline $\mathbf{3 4}$ & Erörterung, mind. 20 Min. & 17,49 & $\mathbf{4 0 , 2 2}$ \\
\hline $\mathbf{6 0}$ & $\begin{array}{l}\text { Konsil zwischen zwei oder mehr liquidationsberechtigten } \\
\text { Ärzten, für jeden Arzt }\end{array}$ & 6,99 & $\mathbf{1 6 , 0 9}$ \\
\hline $\mathbf{7 5}$ & Ausführlicher Krankheits- und Befundbericht & 7,58 & $\mathbf{1 7 , 4 3}$ \\
\hline $\mathbf{8 0}$ & Schriftliches Gutachten & 17,49 & $\mathbf{4 0 , 2 2}$ \\
\hline $\mathbf{8 5}$ & Gutachten, erhöhter Aufwand & 29,14 & $\mathbf{6 7 , 0 2}$ \\
\hline $\mathbf{9 5}$ & Schreibgebühr/Seite $(\mathrm{x} n)$ & $\mathbf{3 , 5 0}(\mathbf{x} \mathbf{n})$ & - \\
\hline $\mathbf{9 6}$ & Kopiergebühr, je Kopie $(\mathrm{x} n)$ & $\mathbf{0 , 1 8}(\mathbf{x} \mathbf{~ n )}$ & - \\
\hline
\end{tabular}

Portokosten nach angefallenen Kosten, s. GOÄ § 10, berechnen.

Für die Nachbefundung, von z. B. Röntgen- oder Sonographiebildern, stehen nur die Beratungsgebühren GOÄ Nrn. 1 oder 3 zur Verfügung.

Um als niedergelassener Arzt sinnvolle Second Opinion leisten zu können, ist ein (guter) Kontakt mit den entsprechenden klinischen Abteilungen nicht nur wertvoll, sondern unserer Meinung nach dringend erforderlich.

\section{Schulungen}

Dem Wunsch der Patienten, bei chronischen Erkrankungen mehr über die eigene Krankheit zu wissen, sollte der Arzt entgegen kommen, denn „mehr Wissen“ verbessert die Compliance. Außerdem wird der Patient sinnvoll in die Therapie mit eingebunden. Bei den Leistungen nach Nrn. 33 und A 36 handelt es sich um Schulungen, die individuell auf den einzelnen Patienten ausgerichtet sind. In den Erläuterungen zur originären Nr. 33 -Diabetikerschulung - schreiben Lang, Schäfer, Stiel und Vogt in ihrem GOÄ-Kommentar zum Inhalt der Leistung: „... Erkennen von Krankheitskomplikationen, Anleitung zum regelmäßigen Überprüfen der Blut- und Harnzuckerwerte, Hinweis zum Einsatz und zur Dosierung von Therapeutika - insbesondere bei Insulin-Selbstmedikation - Anleitung zum Diät- und Ernährungsverhalten..." Diese Bedingungen lassen sich auf zahlreiche chron. Erkrankungen übertragen und bilden damit die Möglichkeit des analogen Ansatzes der Leistungen nach Nrn. 33 oder A 36 (Empfehlung der Bundesärztekammer).

\begin{tabular}{|l|l|r|r|}
\hline GOÄ Nr. & Kurzlegende & $\mathbf{1 f a c h} €$ & $\mathbf{2 , 3 f a c h} €$ \\
\hline $\mathbf{3 3}$ & $\begin{array}{l}\text { Schulung einer Einzelperson, mind. 20 Min. bei } \\
- \text { für Diabetes } \\
- \text { Gestationsdiabetes } \\
- \text { Z. n. Pankreatektomie }\end{array}$ & $\mathbf{1 7 , 4 9}$ & $\mathbf{4 0 , 2 2}$ \\
\hline A 36 & $\begin{array}{l}\text { Schulung einer Einzelperson, mind. 20 Min. bei } \\
- \text { Asthma bronchiale } \\
- \text { Hypertonie - analog GOÄ Nr. 33 }\end{array}$ & 17,49 & $\mathbf{4 0 , 2 2}$ \\
\hline
\end{tabular}

Die Nr. 33 kann nach Meinung der BÄK auch für evaluierte Schulungsprogramme bei

- Chron. rheumat. Erkrankungen

- Osteoporose

- Fibromyalgie-Syndrom

- Chron. Antikoagulanzien-Therapie analog angesetzt werden - s. GOÄ § 6 (2).

Weitere sinnvolle Schulungsindikationen finden Sie in unserer nachfolgenden Auflistung. Nach GOÄ Nr. 33 GOÄ wären auch berechenbar Schulungen des Betroffenen oder seiner nächsten Angehörigen, wie:

- Feldenkrais-Methode - Körperschulung durch Verbesserung der Wahrnehmung

- Ernährungsschulung - Fettstoffwechselstörungen

- Schulung bei Epilepsie 
E. Individuelle Gesundheitsleistungen von A - Z

- Stoffwechselerkrankung bei Kindern

- Raucherentwöhnung

- Alkoholentwöhnung

- Bewegungsschulungen

- Verhaltensschulung

- Schulung bei schweren chronischen Krankheiten, z. B. Malignom, M. Parkinson

Abrechnungstipp: Bei einer Schulung von z. B. 45 Minuten raten die Autoren Lang, Schäfer, Stiel und Vogt in ihrem GOÄ-Kommentar mit einem höheren Gebührensatz über dem Schwellenwert abzurechnen!

\section{Bescheinigungen - Atteste - Gutachten}

Manche Patienten bitten um eine Flut von Bescheinigungen für sich oder Dritte. Dieser Heißhunger auf Geschriebenes ist nur dadurch einzudämmen, dass solche Bescheinigungen liquidiert werden. Dabei ist es selbstverständlich, dass ein Hausarzt bei einer sozial schwachen Familie, z. B. für eine Schulbescheinigung, wenig (1facher Satz) oder nichts berechnen wird.

Für häufig gewünschte Bescheinigungen ist es ratsam, eigene Formblätter im Computer zu gestalten. Dies spart im Praxisalltag Zeit.

\begin{tabular}{|c|c|c|c|}
\hline Kurzlegende & GOÄ-Nr. & 1fach $€$ & $2,3 f a c h €$ \\
\hline Adoptionsgutachten bei Kindern (ggf. + Untersuchung) & 75 & 7,58 & 17,43 \\
\hline $\begin{array}{l}\text { Aufnahmeanträge - Altersheim, Kindergarten (ggf. + Untersu- } \\
\text { chung, für Kindergarten ggf. mit Tine-Test) }\end{array}$ & 75 & 7,58 & 17,43 \\
\hline Arbeitsunfähigkeitsbescheinigung & 70 & 2,33 & 5,36 \\
\hline $\begin{array}{l}\text { Attest für Einreisebehörden z. B. USA über erforderliche im } \\
\text { Gepäck mit geführte med.techn. Geräte und/oder Medikamente } \\
\text { (z. B. Spritzen, Pen, Insuline) - je nach Aufwand }\end{array}$ & $\begin{array}{l}70 \\
75\end{array}$ & $\begin{array}{l}2,33 \\
7,58\end{array}$ & $\begin{array}{r}5,36 \\
17,43\end{array}$ \\
\hline $\begin{array}{l}\text { Bescheinigung, kurze ärztliche z. B. für Schule, Kindergarten, } \\
\text { Sportverein }\end{array}$ & 70 & 2,33 & 5,36 \\
\hline $\begin{array}{l}\text { Behandlungsplan bei tumorkranken Patienten - Chemothe- } \\
\text { rapie und/oder Nachsorge, }\end{array}$ & 78 & 10,49 & 24,13 \\
\hline $\begin{array}{l}\text { Diätplan, individueller schriftlich - bei langfristigen detaillierten } \\
\text { Plänen erscheint ein höherer Steigerungssatz angemessen. } \\
\text { Begründung: Erheblicher, das normale Maß übersteigender } \\
\text { Aufwand, da langfristiger Diätplan }\end{array}$ & 76 & 4,08 & 9,38 \\
\hline Entlassungsbericht im Krankenhaus, vorläufiger & A 72 & 2,33 & 5,36 \\
\hline $\begin{array}{l}\text { Flugtauglichkeitsbescheinigung je nach Aufwand } \\
\text { (ggf. + Untersuchung) }\end{array}$ & $\begin{array}{l}70 \\
75\end{array}$ & $\begin{array}{l}2,33 \\
7,58\end{array}$ & $\begin{array}{r}5,36 \\
17,43\end{array}$ \\
\hline Gesundheitszeugnis z. B. für Visum & 75 & 7,58 & 17,43 \\
\hline Gutachterliche Äußerung, schriftlich + Schrei & 80 & 17,49 & 40,22 \\
\hline $\begin{array}{l}\text { Gutachterliche Äußerung, schriftlich - bei höherem Aufwand } \\
\text { mit wissenschaftlicher Begründung! Je angefangene Stunde } \\
\text { Arbeitszeit + Schreibgebühren }\end{array}$ & 85 & 29,14 & 67,03 \\
\hline Impfbefreiungszeugnis z. B. bei Gelbfieberimpfung & 75 & 7,58 & 17,43 \\
\hline $\begin{array}{l}\text { Kindergartenbescheinigung - Kindergartenunfähigkeit, } \\
\text { Wiedergenesung }\end{array}$ & 70 & 2,33 & 5,36 \\
\hline $\begin{array}{l}\text { Krankheits- und Befundbereicht, schriftlicher - die vom Pa- } \\
\text { tienten oft gewünschte Zusammenfassung der Untersuchungs- } \\
\text { ergebnisse wird von den PKV-Kassen nicht gezahlt. Der Patient } \\
\text { sollte darauf hingewiesen werden! }\end{array}$ & 75 & 7,58 & 17,43 \\
\hline Patientenbuch - Ergänzung von Daten & 70 & 2,33 & 5,36 \\
\hline Reisefähigkeitsbescheinigung (ggf. + Untersuchung) & 70 & 2,33 & 5,36 \\
\hline
\end{tabular}




\begin{tabular}{|c|c|c|c|}
\hline Kurzlegende & GOÄ-Nr. & 1 fach $€$ & $2,3 f a c h €$ \\
\hline $\begin{array}{l}\text { Reiserücktrittsversicherung, Bescheinigung (ggf. + Untersu- } \\
\text { chung) }\end{array}$ & 75 & 7,58 & 17,43 \\
\hline $\begin{array}{l}\text { Schulbescheinigung z. B. über Schulunfähigkeit, Sportunfä- } \\
\text { higkeit }\end{array}$ & 70 & 2,33 & 5,36 \\
\hline $\begin{array}{l}\text { Schwangerschaftsabbruch - Indikation für einen Sch., schrift- } \\
\text { liche Feststellung über das Vorliegen und Nichtvorliegen + } \\
\text { Schreibgebühren }\end{array}$ & 90 & 6,99 & 16,09 \\
\hline $\begin{array}{l}\text { Segel- oder Motorführerschein-Tauglichkeit - Bescheinigung } \\
\text { nach Krankenakte ohne neue Untersuchungen }\end{array}$ & 70 & 2,33 & 5,36 \\
\hline $\begin{array}{l}\text { Sporttauglichkeitsbescheinigung für die Schule } \\
\text { (ggf. + Untersuchung) }\end{array}$ & 70 & 2,33 & 5,36 \\
\hline Sportverein-Bescheinigung (ggf. + Untersuchung) & 70 & 2,33 & 5,36 \\
\hline $\begin{array}{l}\text { Umweltmedizinisches Gutachten } \\
\text { nach Aufwand }\end{array}$ & $\begin{array}{l}80 \\
85\end{array}$ & $\begin{array}{l}17,49 \\
29,14\end{array}$ & $\begin{array}{l}40,22 \\
67,03\end{array}$ \\
\hline $\begin{array}{l}\text { Wehrtauglichkeit-Bescheinigung (ggf. + Untersuchungen) je } \\
\text { nach Umfang }\end{array}$ & $\begin{array}{l}80 \\
85\end{array}$ & $\begin{array}{l}17,49 \\
29,14\end{array}$ & $\begin{array}{l}40,22 \\
67,03\end{array}$ \\
\hline $\begin{array}{l}\text { Schreibgebühr je angefangene DIN A Seite - nur bei Nrn. } 80 \text {, } \\
85,90\end{array}$ & 95 & 3,50 & \\
\hline Kopiergebühr, je Kopie & 96 & 0,18 & \\
\hline
\end{tabular}

Anfallende Portokosten nach GOÄ § 10 berechnen!

Die vorhergehenden aufgelisteten Leistungen sind ggf. um zusätzliche Beratungen und erforderliche Untersuchungen zu erweitern.

\section{Abrechnung von ärztlichen Stellungnahmen im Rahmen von Versicherungsverträgen}

Bei ärztlichen Stellungnahmen im Rahmen von Versicherungsverträgen (z. B. Lebensversicherungs-Begutachtung) hat sich die Praxis der „freien Honorarvereinbarung“ eingebürgert und auch bewährt. Hier wird z. B. vor Abgabe der ärztlichen Stellungnahme von der Versicherung eine Kostenzusage über ein bestimmtes Honorar (z. B. 70,- €) abgegeben.

Ist der Arzt mit diesem Angebot nicht einverstanden, weil er den Arbeitsaufwand größer ansieht, so sollte er dies umgehend der Versicherung schriftlich mitteilen:

„Ich bin gerne zu dem geforderten Gutachten bereit, kann es aber wegen des erheblichen Arbeitsaufwandes nur zu einem Pauschalbetrag von 100,- $€$ erbringen. Ich bitte mir mitzuteilen, ob ich unter diesen Bedingungen das Gutachten erbringen soll."

In der Regel stimmen die Versicherungen zu.

Dieses Abrechnungsverfahren findet außerhalb der GOÄ statt. Rechtliche Basis ist die entsprechende Anwendung des Gesetzes über die Entschädigung von Zeugen und Sachverständigen (ZSEG). Wichtig ist dabei die vertragliche Übereinkunft über den Preis! Eine einseitige Preisfestsetzung seitens einer Versicherung muss ein Arzt nicht akzeptieren.

Pauschale Vereinbarungen dieser Art sind nur mit Versicherungen gestattet.

Ein Pauschalangebot z. B. „Akupunktur Einzelsitzung 30,- Euro“ bei Patienten ist nach ärztlichem Berufsrecht und nach der GOÄ nicht statthaft.

Versicherungsgutachten ohne Untersuchung

\begin{tabular}{|l|l|l|l|}
\hline GOÄ-Nr. & Kurzlegende & 1 fach $€$ & 2,3 fach $€$ \\
\hline
\end{tabular}

\begin{tabular}{|l|l|r|r|}
\hline 1. Versicherungsgutachten ohne Untersuchung*) & \\
\hline $\mathbf{8 0}$ & Schriftl. gutachterliche Äußerung & 17,49 & $\mathbf{4 0 , 2 2}$ \\
\hline $\mathbf{8 5}$ & Gutachten, erhöhter Aufwand & 29,14 & $\mathbf{6 7 , 0 2}$ \\
\hline $\mathbf{9 5}$ & Schreibgebühr je angefangene DIN A Seite & $\mathbf{3 , 5 0}$ & \\
\hline $\mathbf{9 6}$ & Kopiergebühr, je Kopie & $\mathbf{0 , 1 8}$ & \\
\hline
\end{tabular}

*) zuzüglich anfallender Portokosten berechnen (GOÄ § 10) 
E. Individuelle Gesundheitsleistungen von A - Z

Versicherungsgutachten mit Untersuchung

\begin{tabular}{|l|l|r|r|}
\hline GOÄ-Nr. & Kurzlegende & 1fach $€$ & 2,3fach $€$ \\
\hline 2. Versicherungsgutachten mit Untersuchung*) & 4,66 & $\mathbf{1 0 , 7 2}$ \\
\hline $\mathbf{1}$ & Beratung & 15,15 & $\mathbf{3 4 , 8 5}$ \\
\hline $\mathbf{8}$ & Ganzkörperuntersuchung & 29,14 & $\mathbf{6 7 , 0 2}$ \\
\hline $\mathbf{8 5}$ & Gutachten, erhöhter Aufwand & $\mathbf{3 , 5 0}$ & \\
\hline $\mathbf{9 5}$ & Schreibgebühr je angefangene DIN A Seite & $\mathbf{0 , 1 8}$ & \\
\hline $\mathbf{9 6}$ & Kopiergebühr, je Kopie & \\
\hline
\end{tabular}

In der Regel sind für ein Gutachten mit Untersuchung neben der Ganzkörperuntersuchung auch apparative Diagnostik (Ultraschall, EKG, BelastungsEKG, EEG; Rö-Thorax, Doppler etc.) und Laboruntersuchungen erforderlich.

Diese Leistungen können einzeln nach der GOÄ berechnet werden. Meist sind sie aber in einem höheren Pauschalpreis gleich vom Versicherungsunternehmen eingerechnet worden.

\section{Befundbericht für Versicherung ohne gutachterliche Frage*)}

\begin{tabular}{|l|l|r|r|}
\hline $\mathbf{7 5}$ & Schriftlicher Befundbericht & $\mathbf{7 , 5 8}$ & $\mathbf{1 7 , 4 3}$ \\
\hline
\end{tabular}

\begin{tabular}{|l|l|r|r|}
\hline 4. Versicherungsanfrage mit Bitte um gutachterliche Äußerung & 17,49 & $\mathbf{4 0 , 2 2}$ \\
\hline $\mathbf{8 0}$ & Gutachten & $\mathbf{3 , 5 0}$ & \\
\hline $\mathbf{9 5}$ & Schreibgebühr je angefangene DIN A Seite & $\mathbf{0 , 1 8}$ & \\
\hline $\mathbf{9 6}$ & Kopiergebühr, je Kopie & \\
\hline
\end{tabular}

*) zuzüglich anfallender Portokosten berechnen (GOÄ § 10)

\section{Hausbesuche - Wegegeld - Reiseentschädigung}

Viele Familien können die betagten und alleinlebenden Eltern oder nahe Angehörigen nicht ständig besuchen und schon gar nicht pflegen. Sie sind froh über die Hilfe der ambulanten Pflegedienste oder die Betreuung auf einer Pflegestation.

Über den medizinischen Zustand ihrer Angehörigen möchten sie aber regelmäßig mit dem Hausarzt sprechen. In diesem Zusammenhang werden auf Wunsch zusätzliche Hausbesuche durchgeführt, die weder bei GKV- noch bei PKV-Patienten zu Lasten der Krankenkassen abrechenbar sind und dem Patienten im Rahmen von „Wunschbesuchen“ als IGEL-Leistung direkt nach GOÄ zu berechnen sind.

\begin{tabular}{|l|l|r|r|}
\hline GOÄ Nr. & Kurzlegende & $\mathbf{1 f a c h} €$ & ${ }^{\star} \mathbf{1 , 8 / 2 , 3 f a c h} €$ \\
\hline $\mathbf{4 8}$ & Besuch auf Pflegestation - regelmäßig - vereinbarte Zeiten & 6,99 & $\mathbf{1 6 , 0 9}$ \\
\hline $\mathbf{5 0}$ & Besuch & 18,65 & $\mathbf{4 2 , 9 0}$ \\
\hline $\mathbf{5 1}$ & $\begin{array}{l}\text { Besuch eines weiteren Kranken in derselben häuslichen } \\
\text { Gemeinschaft }\end{array}$ & 14,57 & $\mathbf{3 3 , 5 2}$ \\
\hline $\mathbf{5 2}$ & $\begin{array}{l}\text { Aufsuchen eines Patienten außerhalb der Praxisräume oder } \\
\text { des Krankenhauses durch nichtärztliches Personal. Nur mit } \\
\text { 1fachen Gebührensatz berechnungsfähig! }\end{array}$ & 5,83 & - \\
\hline $\mathbf{5 6 *}$ & $\begin{array}{l}\text { Verweilen, ohne Unterbrechung und Erbringung anderer ärzt- } \\
\text { licher Leistungen - je angefangene halbe Stunde }\end{array}$ & 10,49 & $\mathbf{1 8 , 8 9}$ \\
\hline
\end{tabular}

Nach den GOÄ Nrn. 48, 50, 51, 52 und 56 sind z. B. abrechenbar:

- Präventiver Haus- oder Heimbesuch z. B. bei älteren Patienten oder Pflegebedürftigen Ggf. auf Grund der Diagnostik erforderliche Leistungen z. B. Untersuchung bei Herzschmerzen, Verbände bei Wunden etc. können ganz normal nach EBM oder GOÄ abgerechnet werden - je nach Versicherung des Patienten 
Zuschläge ${ }^{1)}$ zu Besuchen in $€$

\begin{tabular}{|l|l|r|}
\hline Zuschlag & Kurzlegende & $\mathbf{1 f a c h} €$ \\
\hline $\mathbf{E}^{1}$ ) & für dringend angeforderte u. unverzüglich erfolgte Ausführung & $\mathbf{9 , 3 3}$ \\
\hline $\mathbf{F}^{1}$ ) & für Leistungen von 20-22 Uhr und 6-8 Uhr & $\mathbf{1 5 , 1 5}$ \\
\hline $\left.\mathbf{G}^{1}\right)$ & für Leistungen von 22-6 Uhr & $\mathbf{2 6 , 2 3}$ \\
\hline $\mathbf{H}^{1}$ ) & für Leistungen an Samstagen, Sonn- und Feiertagen & $\mathbf{1 9 , 8 2}$ \\
\hline K2 $^{1}$ ) & für Leistungen bei Kindern bis zum vollendeten 4.Lebensjahr & $\mathbf{6 , 9 9}$ \\
\hline
\end{tabular}

1) Abrechnung nur mit 1 fachem Satz möglich

Wegegeld* in $€$ (siehe: § 8 GOÄ)

Entfernungen (Radius) von der Praxis

tagsüber

$2 \mathrm{~km} \quad 2-5 \mathrm{~km} \quad 5-10 \mathrm{~km} \quad 10-25 \mathrm{~km}$

nachts (20-8)

\begin{tabular}{llll}
\hline 3,58 & 6,65 & 10,23 & 15,34 \\
7,16 & 10,23 & 15,34 & 25,56
\end{tabular}

\section{Reiseentschädigung ( $\$ 9$ GOÄ)}

Bei mehr als $25 \mathrm{~km}$ erhält der Arzt eine Reiseentschädigung von 0,26 Cents pro km. Bei Abwesenheit bis zu 8 Stunden erhält der Arzt 51,13 Euro, bei Abwesenheit über 8 Stunden 102,26 Euro.

\section{Lifestyle Medizin}

Zu diesem Wunsch-Thema eines Patienten sind primär Beratungen und ggf. auch Untersuchungen erforderlich, denn in nur der Hälfte der Fälle geht es dem Patienten um Fragen zu Medikamenten.Häufiger werden Anti-Aging-Checks für Mann oder Frau nachgefragt.

Abrechnung

\begin{tabular}{|l|l|r|r|}
\hline GOÄ Nr. & Kurzlegende & $\mathbf{1 f a c h} €$ & $\mathbf{2 , 3 f a c h} €$ \\
\hline $\mathbf{1}$ & Beratung & 4,66 & $\mathbf{1 0 , 7 3}$ \\
\hline $\mathbf{3}$ & Eingehende Beratung (10 Min.) nicht neben Sonderleistungen & 8,74 & $\mathbf{2 0 , 1 1}$ \\
\hline
\end{tabular}

Wichtiger Hinweis zur Abrechnung von Spezialloborleistungen (M III und M IV):

Bei Weiterversand von Untersuchungsmaterial durch einen Arzt an einen anderen Arzt wegen der Durchführung von Laboruntersuchungen der Abschnitte M III und/oder M IV hat die Rechnungsstellung durch den Arzt zu erfolgen, der die Laborleistung selbst erbracht hat.

(Aus: Allgemeine Bestimmungen Punkt 3. -Kapitel M Laboratoriumsuntersuchungen)

Möglicher Anti-Aging-Checks für den Mann

\begin{tabular}{|c|c|c|c|}
\hline GOÄ Nr. & Kurzlegende & 1 fach $€$ & ${ }^{\star} 1,15 \mathrm{fach} €$ \\
\hline $250^{*}$ & Blutentnahme i.v. - *1,8facher Satz & 2,33 & 4,20 \\
\hline $3765^{\star}$ & Sexualhormonbindendes Globulin & 26,33 & 30,16 \\
\hline $4021^{*}$ & Follitropin (FSH) & 14,57 & 16,76 \\
\hline 4030* & Thyreoidae stimulierendes Hormon (TSH) & 14,57 & 16.76 \\
\hline 4038* & Dihydroepiandrosteronsulfat & 20,40 & 23,46 \\
\hline 4042 & Testosteron & 20,40 & 23,46 \\
\hline
\end{tabular}

Möglicher Anti-Aging-Checks für die Frau

\begin{tabular}{|l|l|r|r|}
\hline GOÄ Nr. & Kurzlegende & $\mathbf{1 f a c h} €$ & ${ }^{*} \mathbf{1 , 1 5 f a c h} €$ \\
\hline $\mathbf{2 5 0 *}$ & Blutentnahme i.v. - *1,8facher Satz & 2,33 & $\mathbf{4 , 2 0}$ \\
\hline $\mathbf{4 0 2 1}^{*}$ & Follitropin (FSH) & 14,57 & $\mathbf{1 6 , 7 6}$ \\
\hline $\mathbf{4 0 3 9}^{\star}$ & Östradiol & 20,40 & $\mathbf{2 3 , 4 6}$ \\
\hline
\end{tabular}




\section{Impfempfehlungen}

\subsection{Standardimpfungen}

Die Ärzte unterschiedlicher Fachrichtungen werden von Ihren Patienten nach den gängigen Impfungen im Kindes- und Erwachsenenalter und nach erforderlichen Auffrischungsimpfungen gefragt. Die „Neuen Empfehlungen der Ständigen Impfkommission“ erhält der Arzt auf den Seiten des Robert Koch Institutes (http://www.rki.de).

Die Stiko informiert: ... „Die STIKO, die Ständige Impfkommission am Robert Koch-Institut, hat im Epidemiologischen Bulletin 34/2013 den neuen Impfkalender veröffentlicht. Hinzugekommen ist gegenüber dem Impfkalender von 2012 die Empfehlung für eine Rotavirus-Schutzimpfung bei Säuglingen. Die neu empfohlene Rotavirus-Impfung wird als Schluckimpfung gegeben, die Impfserie sollte im Alter von sechs bis zwölf Wochen beginnen und je nach Impfstoff bis zur vollendeten 24. oder 32. Lebenswoche beendet sein. Veränderungen gibt es auch bei den Empfehlungen zur Hepatitis-Bund zur Influenza-Impfung.

Im Epidemiologischen Bulletin 35/2013 sind die Neuerungen in den aktuellen STIKO-Empfehlungen dargestellt. In dieser Ausgabe ist auch die wissenschaftliche Begründung zur Rotavirus-Standardimpfung von Säuglingen erschienen..."

\subsection{Impfungen im Rahmen von Fernreisen - Reisewarnungen}

Wer sich auf eine private oder berufliche Fernreise vorbereitet, denkt zuerst an die erforderlichen Impfungen und fragt in der Regel seinen Hausarzt.

Die gefragten Ärzte können die erforderlichen Impfungen für die verschiedenen Länder nicht alle wissen und bedürfen der Hilfe erfahrener Kollegen der Tropeninstitute oder der Klinikabteilungen für Infektions- und Tropenmedizin in der Bundesrepublik.

Die folgende Auflistung soll die Kontaktaufnahme erleichtern:

\section{Tropeninstitute in Hamburg}

- Bernhard-Nocht-Institut für Tropenmedizin

Bernhard-Nocht-Straße 74

20359 Hamburg

Telefon: +49 40/42 818-0

\section{Tropeninstitute in Nordrhein-Westfalen}

- Tropenmedizinische Ambulanz

Heinrich-Heine-Universität Düsseldorf

Klinik für Gastroenterologie und Infektiologie

Moorenstr. 5

40225 Düsseldorf

Telefon: +49211/81 17031

Fax: +49 211/8118752

- Institut für Medizinische Parasitologie der Universität Bonn

Siegmund-Freud-Straße 25

53127 Bonn

Telefon: +49 228/287-56 73

Tropeninstitute in Schleswig-Holstein

- Zentrum für angewandte Tropenmedizin und Infektionsepidemiologie

Schiffahrtsmedizinisches Institut der Marine

Kopperpahler Allee 120

24119 Kronshagen (bei Kiel)

Telefon: +49 431/-54 09/-17 07/-15 65/- 1453

Fax: +49 431/-54 09/-15 33

Bereitschaftsdienst (ab 16:00 Uhr): +49 431-3 84-0

\section{Tropeninstitute in Berlin}

- Institut für Tropenmedizin Berlin (Charité)

Spandauer Damm 130

14050 Berlin

Telefon: +49 30/301166 
E. Individuelle Gesundheitsleistungen von A-Z

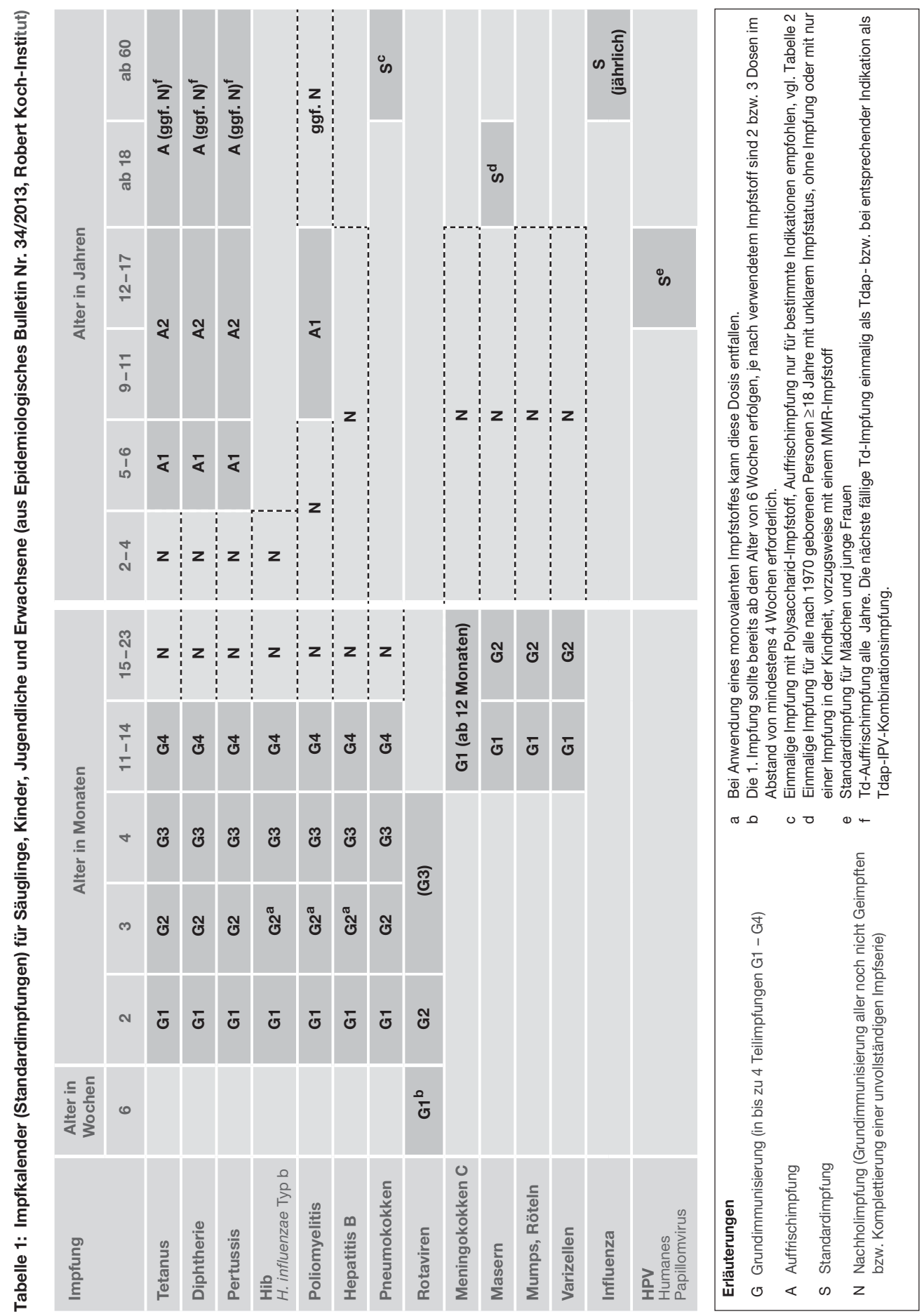


E. Individuelle Gesundheitsleistungen von A - Z

- Universitätsklinikum Rudolf Virchow (Infektiologie Charité)

Standort Wedding

II. Medizinische Abteilung

Augustenburger Platz 1

13353 Berlin

Telefon: +49 30/45 05-0

Tropeninstitute in Mecklenburg-Vorpommern

- Abteilung für Tropenmedizin und Infektionskrankheiten

Klinik und Poliklinik für Innere Medizin der Universität Rostock

Ernst-Heydemann-Straße 6

18057 Rostock

Telefon: +49 381/494-75 11

Fax: +49 381/494 -75 09

Tropeninstitute in Sachsen

- Institut für Tropenmedizin

Städtisches Klinikum Dresden-Friedrichstadt

Friedrichstraße 39

01067 Dresden

Telefon: +49 351/48 03805

- Universitätsklinikum Leipzig

Zentrum für Innere Medizin, Med. Klinik IV

Fachbereich Infektions- und Tropenmedizin

Philipp-Rosenthal-Str. 27

04103 Leipzig

Telefon: +49 341/97 24971

Fax: +49 341/97 24979

- Zentrum für Reise- und Tropenmedizin Leipzig

Städtisches Klinikum St. Georg

II. Klinik für Innere Medizin

Delitzscher Straße 141

04129 Leipzig

Telefon: +49 341/90 92619

Fax: +49 341/90 92630

Tropeninstitute in Baden-Württemberg

- Institut für Tropenhygiene und Öffentliches Gesundheitswesen der Universität Heidelberg Im Neuenheimer Feld 324

69120 Heidelberg

Telefon: +49 6221/56 2905

Fax: +49 6221/56 5948

- Institut für Tropenmedizin Tübingen

Keplerstr. 15

72074 Tübingen

Telefon: +4970712982365

Fax: +497071295267

- Tropenklinik Paul-Lechler-Krankenhaus

Paul-Lechler-Straße 24

72074 Tübingen

Telefon: $+497071 / 2060$

Fax: $+497071 / 22359$

- Medizinische Universitätsklinik und Poliklinik

Abteilung Innere Medizin III

Sektion Infektiologie und Klinische Immunologie

Robert-Koch-Straße 8

89081 Ulm, Oberer Eselsberg

Telefon: +49 731/500 24421

Fax: +49 731/500 24422 


\section{Tropeninstitute in Bayern}

- Abteilung für Infektions- und Tropenmedizin der Ludwig-Maximilians-Universität München Leopoldstr. 5 80802 München

Telefon: +49 89/21 80-35 17

Fax: +49 89/33 6038

- Städtisches Krankenhaus Schwabing

IV. Medizinische Abteilung

Schwerpunkt: Infektions- und Tropenmedizin, Immunschwächeerkrankungen

Kölner Platz 1

80804 München

Telefon: +49 89/30 68-2601

Fax: +49 89/30 68-3910

- Augenklinik der Universität München

Abteilung für Präventiv- und Tropenophthalmologie

Mathildenstraße 8

80336 München

Telefon: +49 89/51 60-38 24

- Tropenmedizinische Abteilung

Missionsärztliche Klinik

Salvatorstraße 7

97074 Würzburg

Telefon: +49 931/7 91-28 21

Fax: +49 931/7 91-24 53

SPIEGEL ONLINE informiert (http://www.spiegel.de/gesundheit/diagnose/fernreisen-impfungen-undschutzmassnahmen-mit-praeventionsplan-a-888181.html)nach Gesprächen mit Experten des Robert Koch Institutes:

Präventionsplan: Richtig impfen und schützen auf Fernreisen - Grundsätzliches zum Impfund Zeitplan (Autor: Frank Patalong) - es wurden nur Ausschnitte übernommen

....,Vor der Reise sollte die reisemedizinische Beratung stehen (Arzt, Tropeninstitute). Hier wird festgelegt, was nötig ist: Je nach Impfung muss das einige Wochen bis Monate vor Reiseantritt geschehen, um einen vollständigen Impfschutz zu erreichen. Die Impfungen sollten spätestens zwei Wochen vor Reiseantritt erledigt sein.

\section{Standardimpfungen: Tetanus, Diphtherie, Polio, Keuchhusten}

Erwachsene haben durch Vorimpfungen oft eine Grundimmunität: Hier ist sogar noch eine kurzfristige Booster-Impfung möglich. Die Impfungen aus Kindertagen wirken aber nicht ewig: Man sollte grundsätzlich auffrischen, denn die Krankheiten, die durch die Standardimpfungen verhütet werden, gibt es weltweit. Fragen Sie Ihren Arzt, was für Sie sinnvoll ist.

\section{Hepatitis A und $B$}

Typ A: Für den mehr als zehn Jahre wirkenden Impfschutz braucht mein zwei Injektionen in einem Abstand von mehreren Monaten (mindestens sechs Monate, Herstellerabhängig). Einen Grundschutz erreicht man aber circa zwei Wochen nach der ersten Dosis: Daraus ergibt sich der Vorlauf vor der Reise.

Typ B: Mindestens drei Injektionen: Zweite Impfung ein Monat, zweite sechs Monate nach Erstinjektion. Grundschutz wird circa zwei Wochen nach Erstinjektion erreicht - Impfung muss folglich mindestens sechs Wochen vor Reiseantritt erfolgen.

\section{Typhus}

Eine Impfung, die man in akuten Risikogebieten mit niedrigen Hygienestandards oder Krankheitswellen braucht: Sie wirkt nur ein bis drei Jahre. Schutz wird nach sieben bis zehn Tagen nach Impfung erreicht, Risiken sollten auch danach gemieden werden: eine völlige Immunisierung wird oft nicht erreicht.

\section{Dengue}

Klingt exotisch, ist es aber nicht - Dengue kommt inzwischen in den warmen Breiten aller Kontinente vor, mehr als 100 Länder sind betroffen. Es ist eine Boomkrankheit mit ständig steigenden Fallzah- 
E. Individuelle Gesundheitsleistungen von A - Z

len (mehr als 50 Millionen Kranke im Jahr). Einen Impfschutz gibt es nicht: Vor Dengue schützt nur eine anständige Mückenprophylaxe.

\begin{abstract}
Malaria
Es gibt über 100 Millionen Kranke im Jahr, trotz zuletzt etwas sinkender Verbreitung. Die ist vor allem der zunehmenden Bekämpfung der krankheitsübertragenden Mücken geschuldet: Auch bei der Malaria ist Mückenprophylaxe absolute Pflicht. Eine Impfung gibt es noch nicht, der Ansteckung kann man aber mit Hilfe einer medikamentösen Prophylaxe (auch: Chemoprophylaxe) mit verschiedenen Mitteln entgegenwirken. Dabei werden im Prinzip die Medikamente, die man auch in der Therapie bei Erkrankung anwenden würde, vorab eingenommen. Bei kurzfristen Reisen und geringerem Risiko wird mitunter geraten, ein Malariamittel als Übergangs- und Soforthilfe mitzuführen. Was hier anzuraten ist (Nebenwirkungen!) ist abhängig von Reiseziel und Person: Geht eine Reise in ein akut malariagefährdetes Land, sollte man mit dem Arzt sprechen.
\end{abstract}

\title{
Gelbfieber
}

Gelbfieber ist vor allem in Afrika verbreitet, aber auch in Süd- und Mittelamerika. Die Virenerkrankung wird durch Mücken übertragen und führt im schlimmsten Fall zum Tod (ca. 15 Prozent aller Fälle). In zahlreichen sub- und tropischen Ländern ist die Impfung Pflicht, in vielen weiteren eine Bedingung für die Einreise, um die Einschleppung zu verhindern. Die Impfung muss mehr als zehn Tage vor Reiseantritt erfolgen und schützt dann für circa zehn Jahre. Vorgenommen werden darf sie allerdings nur von Tropenmedizinern und staatlich zugelassenen Impfstellen (relativ hohes Impfrisiko, deshalb Impfung nur im Pflicht- und akuten Gefährdungsfall): siehe www.frm-web.de

\section{Cholera}

Ist bei Reisen in Gebiete mit akuten Ausbrüchen empfehlenswert, wenn das Risiko einer Exposition absehbar ist - und darunter finden sich immer wieder einmal Traumziele (zurzeit zum Beispiel Haiti und die Dominikanische Republik). Schluckimpfung mit zwei Dosen im Abstand von einer bis sechs Wochen (Herstellerabhängig), Impfschutz ab circa sieben Tagen nach der zweiten Dosis. Vorlauf vor der Reise: zwei bis acht Wochen.

\section{HIV/Aids}

Stellt in vielen armen Weltgegenden, in denen HIV-Medikamente kaum verbreitet sind, ein erhebliches Risiko dar (vor allem Südostasien, Afrika). Die beste Prophylaxe ist Risikovermeidung: Verzicht auf Sex, vor allem mit Prostituierten (und wenn, dann nie ungeschützt!), auf Tätowierungen und Piercings, auf Drogenkonsum unter Nadeleinsatz. In schwachen Volkswirtschaften mit schlechter medizinischer Versorgung und unzureichenden Hygienestandards ist auch dies ein Krankheitsrisiko (Blutkonserven, Injektionsnadeln etc.). Einen Impfschutz gibt es nicht.

\section{Tollwut}

Ohne Behandlung ist Tollwut absolut tödlich. Übertragen wird sie durch Bisse oder Speichel infizierter Wirtstiere, denkbar ist da vom Eichhörnchen bis zum Tiger alles. Eine Impfung kann auch noch nach einem Biss erfolgen, bei Reisen in akute Risikogebiete mit schlechter medizinischer Versorgung ist sie vorab anzuraten: Nötig sind drei Einzelimpfungen in einem Zeitfenster von circa drei bis vier Wochen.

\section{Mückenprophylaxe}

Mücken gehören zu den häufigsten Überträgern ernsthafter Krankheiten in warmen Weltgegenden. Alle Schutzmaßnahmen zielen darauf ab, den Kontakt mit innen zu vermeiden:

- Lange Hosen, lange Ärmel: Im Urlaub nicht das, was man sich wünscht, aber wirksam. Auch Knöchel schützen! In Gegenden mit hoher Krankheitsgefahr durch Mückenübetragung helle Kleidung tragen (bessere Erkennung), Stoff mit speziellem Insektizid imprägnieren.

- Mückenabweisende Mittel (Repellentien) auf alle unbedeckten Stellen der Haut auftragen. Wichtig: Es gibt viele auch tagaktive Mückenarten, Repellentien sind in manchen Regionen auch am Strand angeraten.

- Imprägnierte Moskitonetze über dem Bett nicht vergessen! Wer nicht weiß, ob das Hotel eines bereithält, nimmt sich eines mit.

- Schlafräume mückenfrei machen. Nach Möglichkeit Fenster geschlossen halten, Klimaanlage nutzen. Im Extremfall (oder für "Grundreinigung“) Insektizid einsetzen. Verstecke nicht vergessen: gezielt unter und hinter Möbel sprühen, versteckte Ecken nicht aussparen..." 
Aktuelle Reisewarnungen vom Bundesaußenministerium

Warnungen vor möglichen terroistischen Anschlägen in einzelnen Reiseländern finden sich im Internet beim Bundesaussenministerium (http://www.auswaertiges-amt.de/DE/Laenderinformationen/ 01-Reisewarnungen-Liste_node.html). Ferner findet der User in diesem Portal auch zahlreiche Hinweise zur Reisemedizinischen Vorsorge.

Abrechnung

Die Impfleistung des Arztes umfasst nach dem Robert Koch Institut:neben der Impfung eine erhebliche Beratung:

- Informationen über den Nutzen der Impfung und die zu verhütende Krankheit,

- Hinweise auf mögliche unerwünschte Arzneimittelwirkungen und Komplikationen,

- Erheben der Anamnese und der Impfanamnese einschließlich der Befragung über das Vorliegen möglicher Kontraindikationen,

- Feststellen der aktuellen Befindlichkeit zum Ausschluss akuter Erkrankungen,

- Empfehlungen über Verhaltensmaßnahmen im Anschluss an die Impfung,

- Aufklärung über Beginn und Dauer der Schutzwirkung,

- Hinweise zu Auffrischimpfungen,

- Dokumentation der Impfung im Impfausweis bzw. Ausstellen einer Impf -bescheinigung

\begin{tabular}{|l|l|r|r|}
\hline GOÄ Nr. & Kurzlegende & $\mathbf{1 f a c h} €$ & $\mathbf{2 , 3 f a c h} €$ \\
\hline $\mathbf{1}$ & Beratung & 4,66 & $\mathbf{1 0 , 7 3}$ \\
\hline $\mathbf{3}$ & Eingehende Beratung (10 Min.) nicht neben Sonderleistungen & 8,74 & $\mathbf{2 0 , 1 1}$ \\
\hline $\mathbf{3 7 5}$ & Schutzimpfung, i.m./s.c. - ggf. einschl. Eintragung in Impfpass & 4,66 & $\mathbf{1 0 , 7 2}$ \\
\hline $\mathbf{3 7 6}$ & Schutzimpfung (oral) einschl. beratendem Gespräch & 4,66 & $\mathbf{1 0 , 7 2}$ \\
\hline $\mathbf{3 7 7}$ & Zusatzinjektion bei Parallelimpfung & 2,91 & $\mathbf{6 , 7 0}$ \\
\hline $\mathbf{3 7 8}$ & $\begin{array}{l}\text { Simultanimpfung (gleichzeitig passive und aktive Impfung } \\
\text { gegen Tetanus) }\end{array}$ & 6,99 & $\mathbf{1 6 , 0 9}$ \\
\hline
\end{tabular}

Hinweis:

Neben Nrn. 376, 377, 378 nicht Nrn. 1 oder 2.

- Muss vor einer Impfung eine Untersuchung zur Feststellung der Impffähigkeit durchgeführt werden, so ist diese abrechnungsfähig.

- Mit der Impfung beginnt ein neuer eigener Behandlungsfall.

- Die Kosten für die Impfstoffe können entweder gemäß GOÄ § 10 entsprechend berechnet oder aber die Impfstoffe zu Lasten des Patienten rezeptiert werden

\section{Neuraltherapie}

Unter Neuraltherapie wird die gezielte Behandlung des vegetativen Nervensystems und peripherer Nerven mit einem Lokalanästhetikum verstanden. Dafür sind drei verschiedene Ansatzpunkte möglich:

1. Die Segment- oder lokale Therapie

2. Die Behandlung von Nervenknotenpunkten (Ganglien)

3. Die Behandlung über ein kausales Störfeld

\section{Indikationen}

Als Indikationen für die Neuraltherapie stellt die Internationale medizinische Gesellschaft für Neuraltherapie nach Huneke Regelationstherapie e.V auf ihrer Internetseite (www.neuraltherapie-online.de/index.php?content=indikationen) fest:

Kopf: Kopfschmerzen, Migräne, Kopfdruck, Folgen von Gehirnerschütterungen und Schädelbrüchen wie Schwindel, Gleichgewichtsstörungen oder Epilepsie als Unfallfolge. Arte-riosklerose des Gehirns und Zustand nach Schlaganfall (nur Besserung der geistigen und körperlichen Beweglichkeit). Manche Formen von Kreislaufstörungen, Haarausfall, Trigemi-nusneuralgien, Fazialislähmungen, Schlaflosigkeit.

Augen: Glaukom (Grüner Star) und alle entzündlichen Augenerkrankungen wie Neuritis, Iridozyklitis, Keratitis, Skleritis usw. 
E. Individuelle Gesundheitsleistungen von A - Z

Ohren: Akute und chronische Mittelohrentzündungen, vom Ohr ausgehende Gleichgewichtsstörungen (Meniere), Ohrensausen und andere Ohrgeräusche, Schwerhörigkeit.

Nase: Heuschnupfen, Ozaena (Stinknase), chronischer Schnupfen, Verlust des Geruchsvermögens, Nebenhöhleneiterungen.

Hals: Schilddrüsenvergrößerung (Kropf) mit oder ohne Schilddrüsenüberfunktion (Basedow), aber auch hochgradige Nervosität mit Heulzwang und Angstzuständen. Chronische Mandelentzündungen, ständiges Druck- und Fremdkörpergefühl im Hals. Peitschen-syndrom nach Auffahrunfällen.

Brust: Bronchialasthma, Herzasthma, Angina pectoris, Herzstiche, Herzbeklemmung, Zustand nach Herzinfarkt, Herzneurose (nervöse Beschwerden ohne erkennbare Ursache), Herzmuskelentzündung, Silikose (Staublunge), Emphysem, gewisse Formen der Lungentuberkulose.

Bauch: Leber- und Gallenleiden, Beschwerden nach Gelbsucht, Magen- und Zwölffingerdarmgeschwüre, Erkrankungen der Bauchspeicheldrüse, Magenneurose, chronische Verstopfung, chronischer Durchfall, Kolitis.

Unterleib: Bei Frauen Entzündungen der Gebärmutter, Eileiter und Eierstöcke, Periodenschmerzen, Ausfluß, Krankheiten, die nach Fehlgeburten oder schweren Entbindungen aufgetreten sind, Kinderlosigkeit, sexuelle Störungen, Schwangerschaftserbrechen, Neigung zu Fehlgeburten. Beim Mann Prostata-(Vorsteherdrüsen-) vergrößerungen und -entzündun-gen,Impotenz. Nierenerkrankungen. Blasenleiden wie Reizblase, auch Bettnässen.

Gelenke: Arthrosis deformans (Alters-und Abnutzungserkrankungen mit Zackenbildung).

Wirbelsäule: Zervikalsyndrom, Spondylosis, Osteochondrosis, Bandscheibenschaden.

Muskeln: Bechterewsche Krankheit, Kreuzschmerzen, Alters-Hüftleiden (Cox-arthrose), Hexenschuss, Kniebeschwerden, Gelenk-und Muskelrheuma, Arthritis, Steißbeinschmerzen, Bänderzerrungen, Muskelrisse und deren Folgen. Knochenhauterkrankungen (z. B. Tennisellenbogen) nach Überanstrengung und Unfällen, Amputationsstumpfschmerzen, organische Durchblutungsstörungen an Armen und Beinen, Sudeck'sche Krankheit, Gefäßkrämpfe, Lymphabflussstauungen, Sportverletzungen.

Haut: Chronische Hautleiden (wie Ekzem), Narbenschmerzen, Keloidnarben, Entzündungen aller Art, After-und Scheidenjuckreiz, Hämorrhoiden-Beschwerden, Thrombosen, Furunkel, Schmerzen nach Gürtelrose, Warzen, schlecht heilende Wunden, offene Beine.

Nerven: Neuralgien aller Art, Nervenentzündungen, Ischias, Gemütsveränderungen nach Krankheiten oder Operationen, „nervöse“" Organleiden, Gefühlsstörungen, funktionelle Erkrankungen, Schmerzzustände aller Art, Polyneuropathien.

Allgemeinerkrankungen: Allergien, „vegetative Dystonie“, krankhaft vorzeitiges Altern, Alters- und Abnützungserkrankungen, Leistungsknick, postoperative Krankheiten, Störungen der Hormondrüsen, Wetterfühligkeit und Föhnkrankheit.

Gefäße: Arterielle und venöse Durchblutungsstörungen, Krampfadern, Unterschenkelgeschwüre.

\section{Abrechnung}

\begin{tabular}{|l|l|r|r|}
\hline GOÄ Nr. & Kurzlegende & $\mathbf{1 f a c h} €$ & $\begin{array}{r}{ }^{*} \mathbf{1 , 8} \boldsymbol{\text { f }} \\
\mathbf{2 , 3 f a c h} \boldsymbol{\text { f }}\end{array}$ \\
\hline $\mathbf{2 5 0}$ & Blutentnahme/Vene & 2,33 & $\mathbf{4 , 2 0}$ \\
\hline $\mathbf{2 5 2}$ & Injektion, subkutan, intrakutan, i.m. & 2,33 & $\mathbf{5 , 3 6}$ \\
\hline $\mathbf{2 5 3}$ & Injektion i.v. & 4,08 & $\mathbf{9 , 3 8}$ \\
\hline $\mathbf{2 5 4}$ & Injektion i.a. & 4,66 & $\mathbf{1 0 , 7 2}$ \\
\hline $\mathbf{2 5 4}$ & Ozon (Analogziff. n. § 6) & 4,66 & $\mathbf{1 0 , 7 2}$ \\
\hline $\mathbf{2 5 5}$ & Injektion intraartikulär, perineural & 5,54 & $\mathbf{1 2 , 7 4}$ \\
\hline $\mathbf{2 5 6}$ & Injektion (Periduralraum) & 10,78 & $\mathbf{2 4 , 8 0}$ \\
\hline $\mathbf{2 5 6}$ & Canalis sacralis & 10,78 & $\mathbf{2 4 , 8 0}$ \\
\hline
\end{tabular}




\begin{tabular}{|c|c|c|c|}
\hline GOÄ Nr. & Kurzlegende & 1 fach $€$ & $\begin{array}{r}{ }^{*} 1,8 / \\
2,3 \text { fach } €\end{array}$ \\
\hline 264 & Injektion/Infiltration der Prostata & 6,99 & 16,09 \\
\hline 266 & Intrakutane Reiztherapie (Quaddeln) je Sitzung & 3,50 & 8,04 \\
\hline 267 & Medikament. Infiltration 1 Körperregion & 4,66 & 10,72 \\
\hline 268 & Medikament. Infiltration mehrerer Körperregionen & 7,58 & 17,43 \\
\hline 284 & Eigenbluteinspritzung & 5,25 & 12,07 \\
\hline 285 & Aderlass & 6,41 & 14,75 \\
\hline 286 & gr. Eigenbluteinspritzung & 12,82 & 29,49 \\
\hline 300 & Punktion eines Gelenks, Finger, Zehen & 6,99 & 16,09 \\
\hline 301 & Punktion eines Ellenbogen, Knie, Sternocl., Dornfortsatz & 9,33 & 21,45 \\
\hline 302 & Punktion eines Schulter-oder Hüftgelenks (mit Zeel) & 14,57 & 33,52 \\
\hline 303 & Punktion einer Drüse oder Ganglion/Struma & 4,66 & 10,72 \\
\hline 315 & Punktion eines Organs (Leber, Milz, Hoden) & 14,57 & 33,52 \\
\hline 483 & Lokalanästh. (NNH), Infiltration kl. Bezirke & 2,68 & 6,17 \\
\hline 490 & Incisurae, Art, subcla. & 3,56 & 8,18 \\
\hline 491 & Infiltr. gr. Bezirke, Adnexen & 7,05 & 16,22 \\
\hline 493 & Leitungsanästhesie & 3,56 & 8,18 \\
\hline 494 & Ober-Unterkiefer je -Pudendusananästhesie, endoneural & 7,05 & 16,22 \\
\hline 495 & Leitungsanästhesie, retrobulbär & 7,05 & 16,22 \\
\hline 497 & $\begin{array}{l}\text { Blockade des Truncus sympathicus (lumbaler Grenzstrang) oder } \\
\text { Ganglion stellatum }\end{array}$ & 12,82 & 24,49 \\
\hline 498 & Plexus sacral., Grenzstrang, sympathicus & 17,49 & 40,22 \\
\hline \begin{tabular}{|l|}
$620^{*}$ \\
analog
\end{tabular} & Rheogramm - analog GOÄ Nr. 620* - entsprechend GOÄ § 6 (2) & 8,86 & 15,95 \\
\hline 1498 & Konserv. Behandlg der Mandeln & 2,56 & 5,90 \\
\hline 2599 & Ganglion Gasseri, Sphenopalatium & 13,11 & 30,16 \\
\hline \multicolumn{4}{|c|}{ Impfungen und Testungen } \\
\hline 384 & $\begin{array}{l}\text { Tuberkulinstempeltest, Mendel-Mantoux-Test oder Stempeltest } \\
\text { mit mehreren Antigenen } \\
\text { (Abrechnung des Hautstempeltests zur Feststellung der Reak- } \\
\text { tionslage abgerechnet werden.) }\end{array}$ & 2,33 & 5,36 \\
\hline $\begin{array}{l}388 \\
\text { analog }\end{array}$ & $\begin{array}{l}\text { Reib-, Scratch- oder Skarifikationstest, je Test (Verwendung von } \\
\text { Spenglersanen etc. zur Provokation von Störfeldern) - analog } \\
\text { GOÄ Nr. } 388 \text { - entsprechend GOÄ § } 6 \text { (2) }\end{array}$ & 2,04 & 4,69 \\
\hline 390 & $\begin{array}{l}\text { Intrakutantest, je Test } \\
\text { (Die Intrakutantestung wird zum Ausschluss einer Allergie auf } \\
\text { das zu verwendende Neuraltherapeutikum der Therapie voran- } \\
\text { gestellt.) }\end{array}$ & 3,50 & 8,04 \\
\hline 393 & $\begin{array}{l}\text { Nasaler oder konjunktivaler Provokationstest bds. mit Einzel- } \\
\text { oder Gruppenextrakt, je Test }\end{array}$ & 5,83 & 13,41 \\
\hline
\end{tabular}

Im Rahmen der Neuraltherapie sind natürlich auch alle erforderlichen Beratungs- und Untersuchungsleistungen ansetzbar. 


\section{Schlafapnoe-Diagnostik}

Diagnostik auf Patientenwunsch ohne vorliegende medizinische Indikation

Die Schlafapnoe-Diagnostik führen Ärzte durch, die zum Führen der Gebietsbezeichnung

- Allgemeinmedizin

- Hals-Nasen-Ohren-Heilkunde

- Innere Medizin

- der Facharzt- und Schwerpunktbezeichnung Innere Medizin und Pneumologie

- Kinder- und Jugendmedizin

- Neurologie

- Psychiatrie und Psychotherapie

berechtigt sind oder entsprechende Krankenhaus-Abteilungen.

Für die Vertragsärzte, die Diagnostik und Therapie schlafbezogener Atmungsstörungen durchführen, besteht eine Qualitätssicherungsvereinbarung gemäß § 135 Abs. 2 SGB V zur Diagnostik und Therapie schlafbezogener Atmungsstörungen (http://www.kbv.de/rechtsquellen/2492.html), die auch ein Nicht-Vertragsarzt, der diese Leistungen erbringt, kennen sollte. Der Ablauf einer Stufendiagnostik ist in der Richtlinien des Gemeinsamen Bundesausschusses gemäß $§ 135$ Abs. 1 SGB V definiert.

Indikationen

- Bei Verdacht auf das Vorliegen eines Schlafapnoesyndroms (Atemausetzer, starkes Schnarchen, Müdigkeit)

- Bei schwer einstellbaren arteriellen Hypertonie

- Bei ständiger Müdigkeit

- Einschlafzwang am Tag

- Konzentrationsschwäche

Abrechnung: Kardiorespiratorische Polygraphie - Kleines Schlaflabor*

Nach Beschlüsse des Ausschusses „Gebührenordnung“ der Bundesärztekammer setzt sich der Leistungskomplex der kardiorespiratorischen Polygraphie setzt sich aus folgenden Leistungen zusammen:

\begin{tabular}{|c|c|c|c|}
\hline GOÄ Nr. & Kurzlegende & 1fach $€$ & $\begin{array}{r}{ }^{*} 1,8 / \\
2,3 \text { fach } €\end{array}$ \\
\hline 1 & Beratung & 4,66 & 10,73 \\
\hline 3 & $\begin{array}{l}\text { Eingehende Beratung (10 Min.) nicht neben Sonderleistun- } \\
\text { gen }\end{array}$ & 8,74 & 20,11 \\
\hline $\begin{array}{l}427 \\
\text { analog }\end{array}$ & $\begin{array}{l}\text { Kontrolle der Beatmung unter nCPAP oder BiPAP - (analog } \\
\text { Nr. } 427 \text { GOÄ) - n. Beschluss des Gebührenordnungsau- } \\
\text { schusses der BÄK }\end{array}$ & 8,27 & 20,11 \\
\hline $602^{*}$ & $\begin{array}{l}\text { Oxymetrische Untersuchung(en) (Bestimmung der prozen- } \\
\text { tualen Sauerstoffsättigung im Blut) - ggf. einschl. Bestim- } \\
\text { mung(en) n.Belastung }\end{array}$ & 8,86 & 15,95 \\
\hline $\begin{array}{l}653 \\
\text { analog }\end{array}$ & $\begin{array}{l}\text { EKG über mind. } 6 \text { Std. (s. Leistungskomplex Schlaflabor) - } \\
\text { (analog Nr. } 653 \text { GOÄ)-n. Beschluss des Gebührenordnung- } \\
\text { sauschusses der BÄK }\end{array}$ & 14,75 & 26,54 \\
\hline $\begin{array}{l}714 \\
\text { analog }\end{array}$ & $\begin{array}{l}\text { Kontinuierliche Registrierung der Körperlage mittels Lage- } \\
\text { sensoren über mind. } 6 \text { Stunden }\end{array}$ & 10,49 & 24,13 \\
\hline $\begin{array}{l}5295^{*} \\
\text { analog }\end{array}$ & $\begin{array}{l}\text { Videokontrolle der Korrelation von elektro.physiol. Aufzeich- } \\
\text { nung u. Verhaltensbefund (analog 5295* GÖ̈) - n. Beschlüs- } \\
\text { sen des Ausschusses „Gebührenordnung” der BÄK }\end{array}$ & 13,99 & 25,18 \\
\hline
\end{tabular}

Hinweis der BÄK: Die Voraussetzungen zur Anerkennung der einzelnen Leistungen im Rahmen der kardiorespiratorischen Polygraphie sind dann erfüllt, wenn jeweils eine kontinuierliche Registrierung beziehungsweise Überwachung über eine mindestens sechsstündige Schlafphase erfolgt. Die jeweilige Dokumentation der einzelnen elektrophysiologischen Messdaten sowie der einfache Befundbericht sind mit den in Ansatz gebrachten Gebührenpositionen abgegolten. 
E. Individuelle Gesundheitsleistungen von A - Z

\section{Elektrotherapie}

Arten der Elektrotherapien und ihre Indikationen:

- Dezimeterwellen (hochfrequenteTherapie) Thermotherapie mit hoher Eindringtiefe -s. auch Mikrowellen-Therapie.

- Iontophorese (galvanischer Strom) Einbringung von Arzneistoffen durch die unverletzte Haut

- Interferenzstom Zwei sich kreuzende Stromkreise mit differierenden Wechselströmen dienen zur Erzeugung endogen wirksamer Schwingungen -

- bei Erkrankungen der Muskulatur und des Bindegewebes

- Kurzwellen Therapie (hochfrequenteTherapie) bei

- Muskelverspannungen,

- rheumatischen Erkrankungen

- chron. Arthrose,

- unterstütztend zur Nachbehandlung bei Verletzungen.

- Mikrowellen Therapie (hochfrequenteTherapie) Thermotherapie mit geringer Eindringtiefe, Wärme wird an vorher festgelegte Bereiche unter die Haut gebracht .zur

- Schmerzdämpfung

- Reizstrom (gepulsten Stromformen; Gleichstrom oder niederfrequenten Wechselströme)

- für Muskelkontraktionen oder Stimulierung von Nervenfasern - s. auch TENS

- Stangerbad (Therapie mit Gleichstrom) bei:

- Schmerzen,

- Lähmungen

- Gefäßerkrankungen

- Ultraschall (hochfrequente Vibrationsmassage)

- bei rheumatischen Erkrankungen,

- nach Verletzungen, fördert die Durchblutung, entspannt die Muskeln

- Schmerzenlinderung

- TENS (transkutane elektrische Nervenstimulation) bei

- Muskel- und Skelettschmerzen,

- Tennisarm,

- posttraumatischen Schmerzen,

- Neuralgien,

- Stumpf- und Phantomschmerzen,

- Karzinomschmerzen.

Heute schon oft in Eigenbehandlung des Patienten mit einem eigenen Gerät nach Einweisung des Arztes.

- Zwei- und Vierzellenbad (Therapie mit Gleichstrom) - Hydroelektrisches Teilbad. Anwendung:

- Schmerzlinderung

- Durchblutungsförderndung -s. auch Stangerbad.

Kontraindikationen bei Ultraschall, Kurzwellen- und Mikrowellentherapie:

- Blutungsneigung,

- Entzündungen,

- Thrombose,

- Rückenmarkerkrankungen,

- Tumore,

- Mangelversorgung von Organen.

Zu beachten ist, dass bei einigen Elektrotherapien für die Träger von Herzschrittmachern und Metallimplantaten Kontraindikationen bestehen.

\section{Abrechnung}

\begin{tabular}{|l|l|r|r|}
\hline GOÄ Nr. & Kurzlegende & $\mathbf{1 f a c h} €$ & $\begin{array}{r}{ }^{*} \mathbf{1 , 8 f} / \\
\mathbf{2 , 3 f a c h} €\end{array}$ \\
\hline $\mathbf{1}$ & Beratung - auch telef. & 4,66 & $\mathbf{1 0 , 7 3}$ \\
\hline $\mathbf{3}$ & $\begin{array}{l}\text { Eingehende Beratung (mind. 10 Min.) - nicht neben Sonderleis- } \\
\text { tungen - auch telef. }\end{array}$ & 8,74 & $\mathbf{2 0 , 1 1}$ \\
\hline
\end{tabular}




\begin{tabular}{|c|c|c|c|}
\hline GOÄ Nr. & Kurzlegende & 1 fach $€$ & 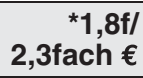 \\
\hline $\begin{array}{l}3211 \\
\text { analog }\end{array}$ & $\begin{array}{l}\text { Anleitung des Patienten zur Selbstanwendung TENS - analog } \\
\text { GOÄ Nr. } 3211 \text { - entsprechend GOÄ § } 6 \text { (2) }\end{array}$ & 6,99 & 16,09 \\
\hline $538^{*}$ & Infarotbehandlung, je Sitzung & 2,33 & 4,20 \\
\hline $539^{*}$ & Kurzwellen-, Mikrowellenbehandlung, je Sitzung & 2,56 & 4,62 \\
\hline $549^{*}$ & $\begin{array}{l}\text { Kurzwellen-, Mikrowellenbehandlung, verschiedene Körperregio- } \\
\text { nen in einer Sitzung }\end{array}$ & 3,21 & 5,77 \\
\hline $551^{*}$ & Reizstrom & 2,80 & 5,04 \\
\hline $552^{*}$ & Iontophorese & 2,56 & 4,62 \\
\hline $553^{*}$ & Vierzellenbad & 2,68 & 4,83 \\
\hline $554^{*}$ & Hydroelektrisches Vollbad & 5,30 & 9,55 \\
\hline $\begin{array}{l}555^{*} \\
\text { analog }\end{array}$ & $\begin{array}{l}\text { 1. TENS - Apparative isokinetische Muskelfunktionstherapie, } \\
\text { je Sitzung } \\
\text { 2. PSP Pulsierende Signaltherapie - analog GOÄ Nr. } 555\end{array}$ & 6,99 & 12,59 \\
\hline
\end{tabular}

\section{Umweltmedizin}

Die Umweltmedizin beschäftigt sich mit den Auswirkungen von Umweltfaktoren auf die Gesundheit der Menschen. Dabei ist es oft schwierig, Krankheitsbilder eindeutig bestimmten Umwelteinwirkungen zuzuordnen.

Besonders bei Ärzten, die Alternative Medizin anbieten, werden von Patienten Schadstoff-Untersuchungen im Sinne der Umweltmedizin gewünscht.

Umweltmedizinische Leistungen gehören in der Regel nicht zum primären Leistungsumfang der GKV und finden sich auch nicht im EBM aufgeführt. Aber es gibt in einzelnen KV-Bezirken Umweltmedizinische Vereinbarungen. Fragen Sie bei Ihrer KV nach, um einen Überblick zu erhalten, welche Leistungen im Rahmen der GKV erbracht werden können und welche Leistungen als sog. IGELLeistungen abzurechnen und vom Patienten selbst zu zahlen sind. In den Vereinbarungen sind die Leistungen, Honorare und ärztlichen Voraussetzungen beschrieben

Wer die Zusatzbezeichnung „Umweltmedizin“ erwerben will, sollte sich bei seiner regionalen Ärztekammer erkundigen, da die formalen Voraussetzungen für die Anerkennung der Zusatzbezeichnung Umweltmedizin unterschiedlich sein können.

Bisher galt, dass diese Bezeichnung von Allgemeinärzten, Arbeitsmedizinern, Dermatologen, HNOÄrzten Internisten, Kinderärzten und Praktischen Ärzten erworben wurden. Inzwischen hat auf dem Klagewege z. B. aber auch ein Urologe, der die Weiterbildung erfolgreich abgeschlossen hatte, die Berechtigung zum Führen der Zusatzbezeichnung erworben.

Als Voraussetzung für das Erbringen und Abrechnen von umweltmedizinischen Leistungen, $z$. $B$. im IGEL-Bereich, nennen viele Autoren in der Regel die Berechtigung zum Führen der Bezeichnung „Facharzt für Hygiene und Umweltmedizin“ oder der Zusatzbezeichnung „Umweltmedizin“. Aber über diese relativ kleine Gruppe hinaus, werden Ärzte unterschiedlicher Fachgebiete im Praxisalltag von ihren Patienten um Leistungen gebeten und erbringen diese auch, denn Umweltmedizin ist ein interdisziplinäres Fach.

Für die Abrechnung stehen folgende Leistungen nach GOÄ zur Verfügung:

1. Beratungs- und Untersuchungsleistungen der jeweiligen Fachrichtung

2. Laborleistungen aus den Bereichen OI bis III

3. Analytisch-toxikologische Laboruntersuchungen von Patientenmaterial - gezielt angeforderte O III Leistungen können nach GKV berechnet werden

4. Therapeutische Maßnahmen.

Viele Labormediziner informieren im Internet über ihre Diagnostik in der Umweltmedizin.

Mögliche Symptome umweltmedizinischer Belastungen: Symptomenkomplexe als Ausdruck einer Gesundheitsbelastung durch Innenraumbelastung mit Pestiziden, Lösungsmittel und ähnlichen Substanzen: 
(Quelle: Ausschnitte vom Umweltinstitut Vitaaktiv: http://www.vitaktiv.de) z. B.:

Schleimhäute:

- Irritation der Nebenhöhlen und Atemwege (z. B. Nasenbluten)

- Irritation des Magen - Darmtraktes

- Irritation der ableitenden Harnwege

Nervensystem:

- Konzentrationsstörungen

- Gedächtnisstörungen

- Schwindel

- Kopfschmerzen

- Störungen psychischer Vorgänge (z. B. depressive Symptome)

- Augenprobleme

- Periphere Nervenstörungen (z. B. verlangsamte NLG)

Immunsystem:

- Allergische Sensibilisierungen (Typ I und Typ IV)

- Dysbalance der Zytokinproduktion in der Zelle

- Autoimmunreaktionen

- Schwächung/oder Überreaktion des Immunsystems

Weitere mögliche allgemeine Symptome:

- Leberbelastung

- Hautveränderungen (z. B. Pigmentierungsstörung)

- Haarausfall

\section{Gesundheitliche Auswirkungen}

Strahlungen, Felder, Schadstoffe und Mikroorganismen haben Einfluss auf viele Körperfunktionen:

- Stoffwechsel und Säure- Basen Haushalt

- Zellteilung- und Wachstum

- Funktion des Immunsystems

- Funktion des Hormonsystems

- Funktion des Herz - Kreislauf - Systems

die Zunahme von umweltbedingten Schadstoffen kann es zu folgenden unerklärlichen Gesundheitsstörungen kommen:

- Schlafstörungen, Müdigkeit, Fatigue Syndrom (FS)

- Migräne und Kopfschmerzen

- Muskel-, Gelenk- und Gliederschmerzen

- Nervosität, Psychosen, Ängste, Depressionen

- Störungen des zentralen Nervensystems (Gehirntumor)

- Hyperaktivität, Lern- und Konzentrationsstörungen

- Hormonelle Störungen, Schwitzen,

- Multiple Chemical Sensitiv Syndrom (MCS)

- sick building Syndrom (Syndrom durch kranke Häuser)

- Allergien, Immunschwäche

- Atemwegserkrankungen, Asthma, Schleimhautreizungen

- Hauterkrankungen, Neurodermitis

Aus umweltanalytischer und umweltmedizinischer Sicht wird beim Auftreten von oben genannten Symptomen, vor allem wenn mehrere gleichzeitig auftreten, dringend angeraten, den Umweltmediziner und Baubiologen zu kontaktieren.

\section{Diagnostik}

Die Ärztekammer Nordrhein veröffentlicht einen Patientenfragebogen zur Umweltanamnese, der durchaus Anwendung in der Praxis finden sollte, da er die Diagnostik ggf. erleichtert.

Nachfolgend finden Sie den Bogen abgedruckt im Internet finden Sie ihn unter: http://www.aekno.de/ downloads/aekno/goae-umwelt-patfragebogen.pdf 
E. Individuelle Gesundheitsleistungen von A - Z

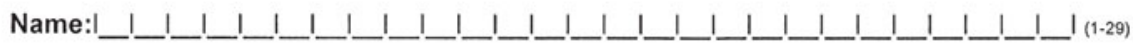

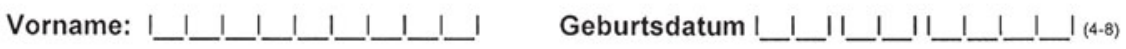

Ausfülldatum: I_I_I_I_I_I_I_I_ (16-21)

\section{Patientenbogen zur Umweltanamnese Netzwerk Umweltmedizin}

\section{Vom Umweltpatienten auszufüllen:}

Bitte füllen Sie die nachfolgenden Fragen gewissenhaft und vollständig aus. Ihre Antworten sind wichtig für das weitere umweltmedizinische Vorgehen. Bei den meisten Fragen sind Antwortmöglichkeiten vorgegeben, von denen Sie eine oder mehrere Antworten ankreuzen können. Wir sind hier an Ihrer persönlichen Einschätzung interessiert. Alle Angaben fallen unter die ärztliche Schweigepflicht.

\section{A. Angaben zur Person}

A1. Alter:

I_I_I_I

$(22 \cdot 25)$

A2. Geschlecht:

$\square 1$ männlich $\square 2$ weiblich

A3. Körpergewicht:

A4. Körpergröße:

$$
\text { I_I_ } \mathrm{cm}
$$

A5. Stellung im Erwerbsleben:

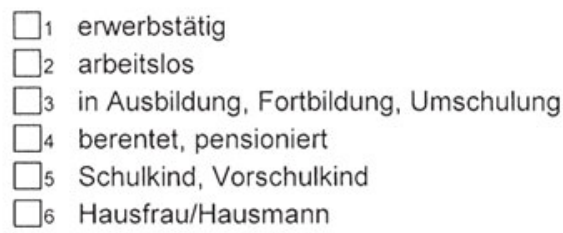

A6. Derzeit bzw. zuletzt ausgeübte berufliche Tätigkeit: 


\section{B. Fragen zu Ihren Beschwerden}

B1. Ich leide derzeitig unter

B3. Die Beschwerden

$$
\begin{array}{ll}
\square & \text { bestehen dauernd } \\
\square & 2 \text { treten zeitweilig auf }
\end{array}
$$

B4. Die Beschwerden

$$
\begin{aligned}
& \square \text { 1 haben sich verschlechtert } \\
& \square 2 \text { haben sich gebessert }
\end{aligned}
$$

B5. Sie werden beeinflußt durch (Mehrfachantworten möglich)

$$
\begin{array}{ll}
\square_{1} & \text { Wetter } \\
\square_{2} & \text { Streß } \\
\square_{3} & \text { Ernährung } \\
\square_{4} & \text { körperliche Belastung } \\
\square_{5} & \text { Aufenthaltsort } \\
\square_{6} & \text { Jahreszeit } \\
\square 7 & \text { Tageszeit } \\
\square 8 & \text { nichts davon }
\end{array}
$$

B6. Sie bessern sich (Mehrfachantworten möglich)

$\begin{array}{ll}\square 1 & \text { im Urlaub/auf Reisen } \\ \square 2 & \text { an Wochenenden } \\ \square 3 & \text { zu Hause } \\ \square 4 & \text { bei der Arbeit } \\ \square & \text { durch Sonstiges: } \\ \square 6 & \text { sie bessern sich nicht }\end{array}$


E. Individuelle Gesundheitsleistungen von A - Z

\section{Liste von Symptomen und Beschwerden}

Im folgenden finden Sie eine Liste von Symptomen und Beschwerden. Nicht alle werden auf Sie zutreffen; in diesen Fällen kreuzen Sie bitte das Feld "tritt nicht auf" an. Wenn einzelne Beschwerden bei Innen auftreten, markieren Sie bitte auf den nebenstehenden Bewertungsskalen

- die Stärke: 1 = gering, 2 = mäßig, 3 = stark (im linken Kasten) und

- die Häufigkeit: 1 = selten, 2 = häufig, 3 = ständig (im rechten Kasten)

\section{C1. HAUT}

Schwellungen, Quaddeln

Rötungen, Ausschläge

Hautverfärbungen

Blässe

Blaue Lippen

Haarausfall

Vermehrter Haarwuchs

Juckreiz

Nagelveränderungen

Schweißausbrüche

\begin{tabular}{c|ccc|ccc|}
\cline { 2 - 6 } $\begin{array}{c}\text { tritt nicht } \\
\text { auf }\end{array}$ & gering & $\begin{array}{c}\text { Stärke } \\
\text { mäßig }\end{array}$ & stark & selten & häufig & ständig \\
$\square 9$ & 1 & 2 & 3 & 1 & 2 & 3 \\
$\square 9$ & 1 & 2 & 3 & 1 & 2 & 3 \\
$\square 9$ & 1 & 2 & 3 & 1 & 2 & 3 \\
$\square 9$ & 1 & 2 & 3 & 1 & 2 & 3 \\
$\square 9$ & 1 & 2 & 3 & 1 & 2 & 3 \\
$\square 9$ & 1 & 2 & 3 & 1 & 2 & 3 \\
$\square 9$ & 1 & 2 & 3 & 1 & 2 & 3 \\
$\square 9$ & 1 & 2 & 3 & 1 & 2 & 3 \\
$\square 9$ & 1 & 2 & 3 & 1 & 2 & 3 \\
$\square 9$ & 1 & 2 & 3 & 1 & 2 & 3 \\
\hline
\end{tabular}

\section{C2. SINNESORGANE}

Sehschwäche

Doppelbilder

Trockene, brennende

Augen

Tränende Augen

Augenrötung

Ohrgeräusche

Verändertes

Tastempfinden

Geschmacksstörungen

Riechstörungen

\begin{tabular}{c|ccc|ccc|}
\cline { 2 - 6 } $\begin{array}{c}\text { tritt nicht } \\
\text { auf }\end{array}$ & \multicolumn{3}{|c|}{ Stärke } & \multicolumn{3}{c|}{ Häufigkeit } \\
$\square_{9}$ & 1 & 2 & 3 & 1 & 2 & 3 \\
$\square_{9}$ & 1 & 2 & 3 & 1 & 2 & 3 \\
$\square_{9}$ & 1 & 2 & 3 & 1 & 2 & 3 \\
$\square_{9}$ & 1 & 2 & 3 & 1 & 2 & 3 \\
$\square_{9}$ & 1 & 2 & 3 & 1 & 2 & 3 \\
$\square_{9}$ & 1 & 2 & 3 & 1 & 2 & 3 \\
$\square_{9}$ & 1 & 2 & 3 & 1 & 2 & 3 \\
$\square_{9}$ & 1 & 2 & 3 & 1 & 2 & 3 \\
$\square_{9}$ & 1 & 2 & 3 & 1 & 2 & 3 \\
\hline
\end{tabular}

(91-117) 


\section{C3. Atemwege}

Laufende Nase, häufiges Niesen

Trockene Nase

Heiserkeit

Verminderte

Nasenatmung

Brennen im Rachen

Reizhusten

Husten mit Auswurf

Asthmaanfälle

Atemnot in Ruhe

Atemnot bei Anstrengung

Häufige Erkältung

\section{C4. HARN- UND GESCHLECHTSORGANE}

Häufiges Wasserlassen nächtl. Wasserlassen

Brennen b. Wasserlassen veränderte Monatsblutung Libido-/Potenzstörung schmerzhafter Geschlechtsverkehr

C5. MusKeln, GLieder

Muskelschwäche

Muskelschmerzen

Wadenkrämpfe

Gelenkbeschwerden

\begin{tabular}{c|ccc|ccc|}
\cline { 2 - 6 } $\begin{array}{c}\text { tritt nicht } \\
\text { auf }\end{array}$ & \multicolumn{3}{c|}{ Stärke } & \multicolumn{3}{c|}{ Häufigkeit } \\
$\square_{9}$ & 1 & 2 & 3 & 1 & 2 & 3 \\
$\square_{9}$ & 1 & 2 & 3 & 1 & 2 & 3 \\
$\square_{9}$ & 1 & 2 & 3 & 1 & 2 & 3 \\
$\square_{9}$ & 1 & 2 & 3 & 1 & 2 & 3 \\
$\square_{9}$ & 1 & 2 & 3 & 1 & 2 & 3 \\
$\square 9$ & 1 & 2 & 3 & 1 & 2 & 3 \\
$\square 9$ & 1 & 2 & 3 & 1 & 2 & 3 \\
$\square 9$ & 1 & 2 & 3 & 1 & 2 & 3 \\
$\square_{9}$ & 1 & 2 & 3 & 1 & 2 & 3 \\
$\square_{9}$ & 1 & 2 & 3 & 1 & 2 & 3 \\
$\square_{9}$ & 1 & 2 & 3 & 1 & 2 & 3 \\
\hline
\end{tabular}

\begin{tabular}{c|ccc|ccc|}
\cline { 2 - 7 } & \multicolumn{3}{|c|}{ Stärke } & \multicolumn{3}{c|}{ Häufigkeit } \\
$\begin{array}{c}\text { tritt nicht } \\
\text { auf }\end{array}$ & gering & mäßig & stark & selten & häufig & ständig \\
$\square 9$ & 1 & 2 & 3 & 1 & 2 & 3 \\
$\square 9$ & 1 & 2 & 3 & 1 & 2 & 3 \\
$\square 9$ & 1 & 2 & 3 & 1 & 2 & 3 \\
$\square 9$ & 1 & 2 & 3 & 1 & 2 & 3 \\
$\square 9$ & 1 & 2 & 3 & 1 & 2 & 3 \\
$\square 9$ & 1 & 2 & 3 & 1 & 2 & 3 \\
\hline$\square$
\end{tabular}

\begin{tabular}{c|ccc|ccc|}
\cline { 2 - 6 } $\begin{array}{c}\text { tritt nicht } \\
\text { auf }\end{array}$ & \multicolumn{3}{|c|}{ Stärke } & \multicolumn{3}{c|}{ Häufigkeit } \\
$\square_{9}$ & 1 & 2 & 3 & 1 & 2 & 3 \\
$\square_{9}$ & 1 & 2 & 3 & 1 & 2 & 3 \\
$\square_{9}$ & 1 & 2 & 3 & 1 & 2 & 3 \\
$\square 9$ & 1 & 2 & 3 & 1 & 2 & 3 \\
\hline
\end{tabular}

(169-180) 
E. Individuelle Gesundheitsleistungen von A - Z

C6. NAHRUNGSAUF-
NAHME UND VERDAUUNG

Mundgeruch

trockener Mund

Zahnfleischveränderung

vermehrter Speichelfluß

Sodbrennen, saures

Aufstoßen

Schluckbeschwerden

Völlegefühl

Übelkeit

Erbrechen

Durchfall

Verstopfung

Blähungen

veränderter Stuhl

Bauchkrämpfe

C7. HERZ- UND
KREISLAUF

Herzrasen

Herzstolpern

Schwellung der Knöchel

Engegefühl in der Brust

"Schwarzwerden" vor den Augen

Schwindel

Ohnmacht

\begin{tabular}{|c|c|c|c|c|c|c|}
\hline \multirow[b]{2}{*}{$\begin{array}{l}\text { tritt nicht } \\
\text { auf }\end{array}$} & \multicolumn{3}{|c|}{ Stärke } & \multicolumn{3}{|c|}{ Häufigkeit } \\
\hline & gering & mäßig & stark & selten & häufig & ständig \\
\hline$\square_{9}$ & 1 & 2 & 3 & 1 & 2 & 3 \\
\hline$\square 9$ & 1 & 2 & 3 & 1 & 2 & 3 \\
\hline$\square 9$ & 1 & 2 & 3 & 1 & 2 & 3 \\
\hline$\square 9$ & 1 & 2 & 3 & 1 & 2 & 3 \\
\hline$\square 9$ & 1 & 2 & 3 & 1 & 2 & 3 \\
\hline$\square 9$ & 1 & 2 & 3 & 1 & 2 & 3 \\
\hline$\square 9$ & 1 & 2 & 3 & 1 & 2 & 3 \\
\hline$\square 9$ & 1 & 2 & 3 & 1 & 2 & 3 \\
\hline$\square 9$ & 1 & 2 & 3 & 1 & 2 & 3 \\
\hline$\square 9$ & 1 & 2 & 3 & 1 & 2 & 3 \\
\hline$\square_{9}$ & 1 & 2 & 3 & 1 & 2 & 3 \\
\hline$\square 9$ & 1 & 2 & 3 & 1 & 2 & 3 \\
\hline$\square_{9}$ & 1 & 2 & 3 & 1 & 2 & 3 \\
\hline$\square_{9}$ & 1 & 2 & 3 & 1 & 2 & 3 \\
\hline
\end{tabular}

(181-222)

\begin{tabular}{|c|c|c|c|c|c|c|}
\hline \multirow[b]{2}{*}{$\begin{array}{l}\text { tritt nicht } \\
\text { auf }\end{array}$} & \multicolumn{3}{|c|}{ Stärke } & \multicolumn{3}{|c|}{ Häufigkeit } \\
\hline & gering & mäßig & stark & selten & häufig & ständig \\
\hline$\square_{9}$ & 1 & 2 & 3 & 1 & 2 & 3 \\
\hline$\square 9$ & 1 & 2 & 3 & 1 & 2 & 3 \\
\hline$\square_{9}$ & 1 & 2 & 3 & 1 & 2 & 3 \\
\hline$\square_{9}$ & 1 & 2 & 3 & 1 & 2 & 3 \\
\hline$\square_{9}$ & 1 & 2 & 3 & 1 & 2 & 3 \\
\hline$\square 9$ & 1 & 2 & 3 & 1 & 2 & 3 \\
\hline$\square_{9}$ & 1 & 2 & 3 & 1 & 2 & 3 \\
\hline
\end{tabular}


C8. StoffFEChSEL

Gewichtszunahme

Gewichtsabnahme

Heißhunger

Vermehrtes Durstgefühl

Kälteempfindlichkeit

Hitzewallungen

Alkoholunverträglichkeit

\begin{tabular}{c|ccc|ccc|}
\cline { 2 - 7 } $\begin{array}{c}\text { tritt nicht } \\
\text { auf } \\
\square_{9}\end{array}$ & gering & $\begin{array}{c}\text { Stärke } \\
\text { mäßig }\end{array}$ & stark & selten & Häufigkeit \\
$\square_{9}$ & 1 & 2 & 3 & 1 & 2 & 3 \\
$\square_{9}$ & 1 & 2 & 3 & 1 & 2 & 3 \\
$\square_{9}$ & 1 & 2 & 3 & 1 & 2 & 3 \\
$\square 9$ & 1 & 2 & 3 & 1 & 2 & 3 \\
$\square 9$ & 1 & 2 & 3 & 1 & 2 & 3 \\
$\square 9$ & 1 & 2 & 3 & 1 & 2 & 3 \\
$\square 9$ & 1 & 2 & 3 & 1 & 2 & 3 \\
\hline
\end{tabular}

C9. Allgemeinbefinden

Appetitverlust

Appetitsteigerung

Tritt nicht

Vermehrte Müdigkeit

Durchschlafstörungen

Antriebsminderung

Mattigkeit

Leistungsverlust

Klossgefühl im Hals

Stimmungslabilität

Vermehrte Reizbarkeit

$$
\text { auf }
$$

Depression

Kopfschmerzen

Einschlafstörungen

Gedächtnisstörungen

Verwirrtheit

Sprachstörungen

Krämpfe

Gleichgewichtsstörungen

Temperaturmißempfinden

Kalte, feuchte Hände

Taubheit der Glieder

Kribbeln der Haut

Zittern

Lähmungserscheinungen

Brennen an

Händen/Füßen
Stärke

$\square 9$

$\square 9$

$\square 9$

$\square 9$

$\square 9$

$\square 9$

$\square 9$

$\square 9$

$\square 9$

$\square 9$

gering

mäßig

2

2$$
2
$$$$
2
$$$$
2
$$$$
2
$$

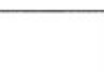

stark

3

3

3

3

3

3

3

3

3

3

प9

口9

प9

$\square 9$

口9

प9

口9

口9

प9

口9

口9

口9

口9

ㅁ9

$\square 9$

3

3

3

3

1

2

\section{Häufigkeit}

selten häufig ständig

$\begin{array}{ll}1 & 2\end{array}$

$\begin{array}{lll}1 & 2 & 3\end{array}$

$\begin{array}{lll}1 & 2 & 3\end{array}$

$\begin{array}{lll}1 & 2 & 3\end{array}$

$\begin{array}{lll}1 & 2 & 3\end{array}$

$\begin{array}{lll}1 & 2 & 3\end{array}$

$\begin{array}{lll}1 & 2 & 3\end{array}$

$\begin{array}{lll}1 & 2 & 3\end{array}$

$\begin{array}{lll}1 & 2 & 3\end{array}$

$\begin{array}{lll}1 & 2 & 3\end{array}$

\begin{tabular}{l|lll}
3 & 1 & 2 & 3
\end{tabular}

\begin{tabular}{l|lll}
3 & 1 & 2 & 3
\end{tabular}

\begin{tabular}{l|lll}
3 & 1 & 2 & 3
\end{tabular}

\begin{tabular}{l|lll}
3 & 1 & 2 & 3
\end{tabular}

\begin{tabular}{l|lll}
3 & 1 & 2 & 3
\end{tabular}

\begin{tabular}{l|lll}
3 & 1 & 2 & 3
\end{tabular}

$\begin{array}{llll}3 & 1 & 2 & 3\end{array}$

\begin{tabular}{l|lll}
3 & 1 & 2 & 3
\end{tabular}

$\begin{array}{llll}3 & 1 & 2 & 3\end{array}$

$\begin{array}{llll}3 & 1 & 2 & 3\end{array}$

3

1

-261) 
E. Individuelle Gesundheitsleistungen von A - Z

\section{Hinweise auf Innenraumbelastungen}

D1. Als Ursache meiner Beschwerden vermute ich:

D2. In meiner Wohnung und meinem Wohnumfeld fühle ich mich

$\begin{array}{ll}\square 1 & \text { sehr wohl } \\ \square & \text { wohl } \\ \square 3 & \text { mäßig wohl } \\ \square & \text { unwohl }\end{array}$

D3. In meiner Wohnung fühle ich mich (Mehrfachantworten möglich)



D4. Eine einmalig hohe Schadstoffeinwirkung in meinem Wohnbereich

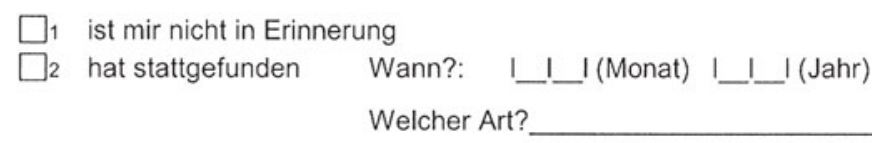

D5. Allergische Reaktionen sind bei mir bereits bekannt auf

$\begin{array}{ll}\square_{1} & \text { Pollen } \\ \square_{2} & \text { Milben } \\ \square_{3} & \text { Tierhaare } \\ \square_{4} & \text { Schimmelpilze } \\ \square_{5} & \text { Nahrungsmittel } \\ \square_{6} & \text { Arzneimittel } \\ \square_{7} & \text { Kosmetika/Pflegemittel } \\ \square_{8} & \text { Duftstoffe } \\ \square_{9} & \text { Metalle } \\ \square_{1} & \text { Insektizide } \\ \square_{2} & \text { Sonstiges: }\end{array}$
(Mehrfachantworten möglich)

$\square_{3}$ nicht bekannt 
D6. Ich habe meine Wohnung in den letzten 12 Monaten verändert durch: (Mehrfachantworten möglich)

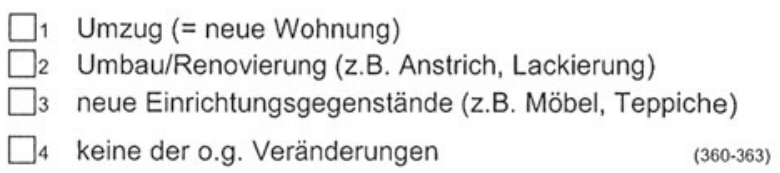

D7. Ich habe Haustiere

D8. In meiner Wohnung gibt es sichtbaren Schimmelpilzbefall oder feuchte Stellen:

$$
\begin{aligned}
& \square 1 \text { nein } \\
& \square 2 \text { ja }
\end{aligned}
$$

D9. In meiner Wohnung wurden in den letzten Jahren Insektenvernichtungs-/

$\begin{array}{ll}\text { Pflanzenschutzmittel verwendet: } & \square 1 \text { nein } \\ & \square_{2} \text { ja } \\ & \square 3 \text { weiß nicht }\end{array}$

\section{E. Fragen zur Medikamenteneinnahme und zum Gesundheitszustand}

\begin{tabular}{|c|c|c|c|}
\hline Medikament & $\begin{array}{c}\text { seit wieviel } \\
\text { Jahren }\end{array}$ & $\begin{array}{c}\text { regelmäßig } \\
\square 1\end{array}$ & $\begin{array}{c}\text { häufig } \\
\square 2\end{array}$ \\
\hline & 111 & $\square 1$ & $\square 2$ \\
\hline & $1 \ldots 1$ & $\square 1$ & $\square_{2}$ \\
\hline & I_I_I & $\square 1$ & $\square 2$ \\
\hline & 111 & $\square_{1}$ & $\square_{2}$ \\
\hline & $\begin{array}{lll}1 & 1 & 1\end{array}$ & $\square 1$ & $\square_{2}$ \\
\hline
\end{tabular}

E1. Welche Medikamente (auch Schlaf, Schmerz-, Abführ-, Stärkungsmittel) nehmen Sie ein?

E2. Sind bei Ihnen noch weitere Erkrankungen (außer den o.g. Beschwerden) bekannt?

$$
\begin{aligned}
& \square 1 \text { nein } \\
& \square 2 \text { ja, und zwar: }
\end{aligned}
$$

E3. Hatten Sie in den letzten fünf Jahren einen Unfall?

$$
\begin{aligned}
& \square 1 \text { nein } \\
& \square 2 \text { ja, Art der Verletzung: }
\end{aligned}
$$


E. Individuelle Gesundheitsleistungen von A - Z

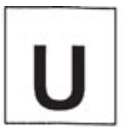

E4. Sind Sie in den letzten fünf Jahren operiert worden?

$\square_{1}$ nein

$\square 2$ ja, Art der Operation:

(387)

\section{F. Fragen zu Ihren Lebens- und Ernährungsgewohnheiten}

F1. Mein Tagesablauf ist: $\square 1$ regelmäßig

$\square 2$ unregelmäßig, wegen:

F2. Ich treibe Sport/mache Bewegungsausgleich:

$\begin{array}{ll}\square 1 & \text { nein } \\ \square & \text { ja, aber selten } \\ \square & \text { ja, regelmäßig }\end{array}$

F3. Rauchen Sie? $\square_{1}$ nein, noch nie

$\square_{2}$ in der Vergangenheit Exraucher seit: I I I Jahren

$\square 3$ ja, gelegentlich

$\square_{4}$ ja, regelmäßig

Anzahl pro Tag: I_I_ I

(390-392)

F4. In meiner Gegenwart wird viel geraucht:

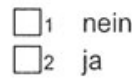

(393)

F5. Von den nachfolgend aufgeführten Getränken trinke ich im Durchschnitt folgende Mengen pro Tag:

\begin{tabular}{|c|c|c|}
\hline Kaffee & I_I I & Tassen \\
\hline Tee & 111 & Tassen \\
\hline Cola & 1 I_ & Gläser/Dosen \\
\hline Bier & 111 & 0,51 Flaschen \\
\hline Wein & 111 & Gläser \\
\hline
\end{tabular}

(394-403)

F6. Haben Sie besondere Ernährungsgewohnheiten (z.B. Diät, Vegetarier)?

F7. Ich lebe: $\square 1$ allein $\square 2$ in Gemeinschaft mit anderen, nämlich zu: I Personen 


\section{Abrechnung}

Empfehlung der Ärztekammer Nordrhein zur Abrechnung ärztlicher umweltmedizinischer Leistungen nach der Gebührenordnung für Ärzte (GOÄ)

...,.,In der gegenwärtigen Situation werden zunehmend umweltmedizinische Leistungen nachgefragt und auch qualitativ hochwertig erbracht. Aus Gründen der Rechtssicherheit sowohl für die Auftraggeber, wie auch für die ärztlichen Auftragnehmer ist es notwendig, dass die erbrachten ärztlichen umweltmedizinischen Leistungen angemessen vergütet werden. Grundlage hierzu ist die GOÄ, soweit keine anderen gesetzlichen Vereinbarungen bestehen. Empfehlungen der Bundesärztekammer zur Abrechnung umweltmedizinischer Leistungen liegen derzeit nicht vor..."

\section{Umweltmedizinische Grundleistungen}

Auf der Basis der Empfehlungen der Ärztekammer Nordrhein (erweitert durch die Autoren in kursiver Schrift) finden Sie einen Abrechnungsvorschlag - unter Hinweis in der Legende finden Sie die Original-Anmerkungen der Ärztekammer Nordrhein

\section{Beratungen}

\begin{tabular}{|c|c|c|c|}
\hline GOÄ Nr. & Kurzlegende & 1 fach $€$ & 2,3 fach $€$ \\
\hline 1 & $\begin{array}{l}\text { Beratung - auch mittels Telefon } \\
\text { Hinweis: Umweltmedizinische Beratung, auch telef., ggf. inkl. } \\
\text { der Zusendung von Informationsmaterial und/oder des umwelt- } \\
\text { medizinischen Patientenfragebogens mit Begleitbrief }\end{array}$ & 4,66 & 10,72 \\
\hline 3 & $\begin{array}{l}\text { Eingehende das gewöhnliche Maß übersteigende Beratung - } \\
\text { auch mittels Fernsprecher - (Mindestdauer } 10 \text { min) } \\
\text { Die Leistung nach Nummer } 3 \text { (Dauer mindestens } 10 \text { Minuten) ist } \\
\text { nur berechnungsfähig als einzige Leistung oder im Zusammen- } \\
\text { hang mit einer Untersuchung nach den Nummern 5, 6, 7, 8, 800 } \\
\text { oder } 801 \text {. Eine mehr als einmalige Berechnung im Behandlungs- } \\
\text { fall bedarf einer besonderen Begründung. } \\
\text { Hinweis: } \\
\text { Umweltmedizinisches Beratungsgespräch unter Auswertung des } \\
\text { Patientenfragebogens zur Umweltanamnese, ggf. unter Bewer- } \\
\text { tung von Vorbefunden und Berücksichtigung spezifischer, biologi- } \\
\text { scher Wirkung von Umweltfaktoren, mit einer Dauer von mehr als } \\
10 \text { min. } \\
\text { Eine deutlich längere Gesprächsdauer kann über den Gebühren- } \\
\text { rahmen (Faktor 2,4-3,5) berücksichtigt werden. } \\
\text { Bei außergewöhnlich langer Gesprächsdauer besteht im Einzel- } \\
\text { fall nach persönlicher Absprache die Möglichkeit einer Honorar- } \\
\text { vereinbarung gemäß § } 2 \text { GOÄ mit Überschreiten des } 3,5 \text { fachen } \\
\text { Faktors. }\end{array}$ & 8,74 & 20,11 \\
\hline $\begin{array}{l}34 \\
\text { analog }\end{array}$ & $\begin{array}{l}\text { Eingehende umweltmedizinische Erörterung - analog GOÄ } \\
\text { Nr. } 34-\text { GOÄ \& } 6 \text { (2) GOÄ Kurztext Nr. 34: Erörterung (Dauer } \\
\text { mindestens } 20 \text { Minuten) } \\
\text { der Auswirkungen einer Krankheit auf die Lebensgestaltung }\end{array}$ & 17,49 & 40,22 \\
\hline
\end{tabular}

\section{Spezielle Anamnese}

\begin{tabular}{|l|l|r|r|}
\hline GOÄ Nr. & Kurzlegende & 1fach $€$ & $\mathbf{2 , 3 f a c h} €$ \\
\hline $\begin{array}{l}\text { 30 } \\
\text { analog }\end{array}$ & $\begin{array}{l}\text { Umweltmedizinische Erstanamnese - analog GOÄ Nr. 30- } \\
\text { GOÄ § 6 (2)-GOÄ Kurztext Nr. 30: Erhebung der homöopathi- } \\
\text { schen Erstanamnese mit einer Mindestdauer von einer Stunde... }\end{array}$ & 52,46 & $\mathbf{1 2 0 , 6 5}$ \\
\hline $\begin{array}{l}\text { 31 } \\
\text { analog }\end{array}$ & $\begin{array}{l}\text { Folgeanamnese - analog GOÄ Nr. 31-GOÄ § 6 (29 } \\
\text { GOÄ Kurztext Nr. 31: Homöopathische Folgeanamnese } \\
\text { mit einer Mindestdauer von 30 Minuten. . }\end{array}$ & 26,23 & $\mathbf{6 0 , 3 3}$ \\
\hline
\end{tabular}


Untersuchungen

\begin{tabular}{|l|l|r|r|}
\hline GOÄ Nr. & Kurzlegende & $\mathbf{1 f a c h} €$ & $\mathbf{2 , 3 f a c h} €$ \\
\hline $\mathbf{8}$ & $\begin{array}{l}\text { Ganzkörperstatus } \\
\text { Hinweis: } \\
\text { Körperliche umweltmedizinische Untersuchung - Ganzkörpersta- } \\
\text { tus (umwelt-medizinisch immer vollständig inkl. orientierender } \\
\text { neurologischer Prüfung) mit Dokumentation bei besonderer Be- } \\
\text { rücksichtigung spezifischer, biologischer Wirkung von Umwelt- } \\
\text { faktoren }\end{array}$ & $\mathbf{3 4 , 8 6}$ \\
\hline
\end{tabular}

\section{Bericht}

\begin{tabular}{|c|c|c|c|}
\hline GOÄ Nr. & Kurzlegende & 1 fach $€$ & $2,3 f a c h €$ \\
\hline 75 & Ausführlicher schriftlicher Krankheits- und Befundbericht & & \\
\hline 80 & $\begin{array}{l}\text { Schriftlich gutachterliche Äußerung } \\
\text { Hinweis } \\
\text { Umweltmedizinischer Bericht (bis } 2 \text { Seiten DIN A 4) mit einer um- } \\
\text { weltmedizinischen gutachterlichen Beurteilung - ggf. unter Ein- } \\
\text { beziehung umweltmedizinischer Messergebnisse - Ein deutlich } \\
\text { längerer Bericht kann über den Gebührenrahmen (Faktor 2,4 - } \\
\text { 3,5) berücksichtigt werden. }\end{array}$ & 17,49 & 40,22 \\
\hline 95 & Schreibgebühr, je angefangene DIN A 4-Seite & $3,50 €$ & \\
\hline 96 & Schreibgebühr, je Kopie & $0,17 €$ & \\
\hline
\end{tabular}

\section{Vorortbegehung}

Die ärztliche Vorortbegehung erfordert spezielle fachübergreifende Kenntnis und eine geeignete Ausstattung, eine Qualitätssicherung mit Zertifizierung sollte vorliegen (ISO 9002 2000),

\begin{tabular}{|c|c|c|c|}
\hline GOÄ Nr. & Kurzlegende & 1 fach $€$ & $\begin{array}{r}{ }^{*} 1,8 / \\
2,3 \mathrm{fach} €\end{array}$ \\
\hline 50 & $\begin{array}{l}\text { Besuch, einschl. Beratung und symptombezogener Untersu- } \\
\text { chung + Wegegeld GOÄ § } 8 \\
\text { Hinweis: } \\
\text { Vorortbegehung mit dem Patienten, bei Auffälligkeit mit Foto- } \\
\text { dokumentation. Eine längere Begehung (z. B. mehr als } 30 \text { Minu- } \\
\text { ten) über den Gebührenrahmen (Faktor } 2,4-3,5) \text { berücksichti- } \\
\text { gen. }\end{array}$ & 18,65 & 42,90 \\
\hline $\begin{array}{l}651^{*} \\
\text { analog }\end{array}$ & $\begin{array}{l}\text { Messung der raumklimatischer Parameter und der Material- } \\
\text { feuchte, - analog Nr. } 651^{\star} \text { gemäß GOÄ } \S 6(2)-\text { Elektrokardio- } \\
\text { graphische Untersuchung in Ruhe - auch ggf. nach Belastung - } \\
\text { mit Extremitäten- und Brustwandableitungen (mindestens neun } \\
\text { Ableitungen)“ } \\
\text { Hinweis: } \\
\text { Messung der raumklimatischer Parameter und der Materialfeuch- } \\
\text { te, ggf. einschließlich Probenahme und dem Freilegen von Bau- } \\
\text { materialien }\end{array}$ & 14,75 & 26,54 \\
\hline 80 & $\begin{array}{l}\text { Schriftlich gutachterliche Äußerung } \\
\text { Hinweis } \\
\text { Schriftlicher Ergebnisbericht mit gutachterlicher Beurteilung und } \\
\text { ggf. mit Darstellung des weiteren Vorgehens. }\end{array}$ & 17,49 & 40,22 \\
\hline
\end{tabular}




\begin{tabular}{|l|l|r|r|}
\hline GOÄ Nr. & Kurzlegende & 1 fach $€$ & $\begin{array}{r}* 1,8 / \\
2,3 f a c h ~ \\
\end{array}$ \\
\hline 95 & Schreibgebühr, je angefangene DIN A 4-Seite & 3,50 & \\
\hline 96 & Schreibgebühr, je Kopie & 0,17 & \\
\hline
\end{tabular}

Porto: Sachkosten gemäß § 10 GOÄ

Umweltmedizinische Analytik

Das Medizinisches Labor Bremen (http://www.mlhb.de/umweltmedizinischea.html) informiert übersichtlich zur Umweltmedizinischen Analytik:

Umweltmonitoring:

Beurteilung der äußeren Belastung durch Nachweis und quantitative Bestimmung von Noxen in der Umwelt.

\section{Biomonitoring:}

Beurteilung der individuellen inneren Belastung durch Nachweis und quantitative Bestimmung von Noxen oder ihren Metaboliten in Körpermaterialien.

\section{Biochemisches Effektmonitoring:}

Nachweis von Einflüssen auf biochemische Vorgänge durch toxische Umweltchemikalien.

\section{Empfänglichkeits-Monitoring:}

Nachweis individueller Faktoren, die die Reaktion auf Umweltnoxen (Höhe der inneren Belastung, Ausmaß der biochemischen Effekte und der gesundheitlichen Störungen) beeinflussen.

Hinweis: Eine vollständige Zusammenfassung aller Umweltgifte, deren Substanzen und relevante Untersuchungsparameter finden Sie auf den Internetseiten fast aller großen medizinischen Labore.

Hinweis: Weitere zur Diagnose führende Sonderleistungen, einschließlich Laborleistungen, sind den entsprechenden Kapiteln der GOÄ zu entnehmen z.B.

Elementeinzelanalysen in Serum, Vollblut, Haaren, Speichel, Urin

\begin{tabular}{|l|c|}
\hline Untersuchungen & GOÄ-Nrn. \\
\hline Calcium & $3555^{\star}$ \\
\hline Kalium & $3557^{*}$ \\
\hline Natrium & $3558^{\star}$ \\
\hline Eisen & $3620^{\star}$ \\
\hline Magnesium & $3621^{*}$ \\
\hline Mangan & $4133^{\star}$ \\
\hline Selen & $4134^{\star}$ \\
\hline Zink & $4135^{*}$ \\
\hline Aluminium & $4190^{\star}$ \\
\hline Arsen & $4191^{*}$ \\
\hline Blei & $4192^{\star *}$ \\
\hline Cadmium & $4193^{\star}$ \\
\hline Chrom & $4194^{*}$ \\
\hline Gold & $4195^{*}$ \\
\hline Quecksilber & $4196^{\star}$ \\
\hline Thallium & $4197^{*}$ \\
\hline $\begin{array}{l}\text { Antimon, Beryllium, Cobalt, Fluor, Gallium, Indium, Jod, Iridium, Mo- } \\
\text { Iybdän, Nickel, Palladium, Platin, Silizium, Silber, Strontium, Titan, Ti- } \\
\text { tan, Vanadium, Wismut, Zinn - für jedes Element }\end{array}$ & $4198^{\star}$ \\
\hline
\end{tabular}




\section{E. Individuelle Gesundheitsleistungen von A - Z}

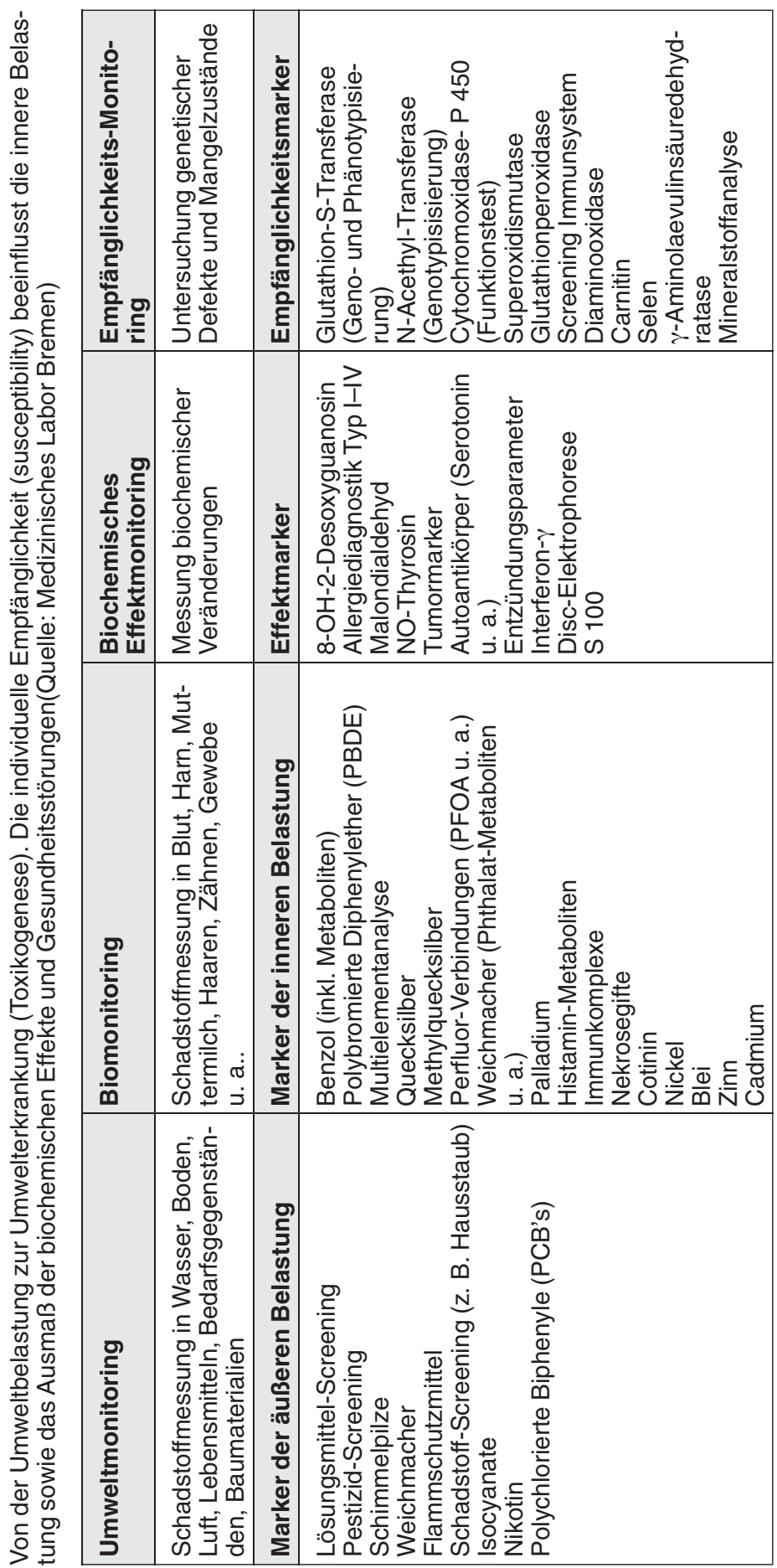


Pflanzen und Holzschutzmittel

\begin{tabular}{|l|c|}
\hline Untersuchungen & GOÄ-Nrn. \\
\hline $\begin{array}{l}\text { Holzschutzmittel im Blut (Hexachlorbenzol, Lindan, PCP, DDT, Endosul- } \\
\text { fan, Methoxychlor, Furmecyclox) }\end{array}$ & $7 \times 4209^{\star}$ \\
\hline Holzschutzmittel im Urin (Pyrethroid-Metaboliten, PCP) & $\begin{array}{c}4210^{*} \text { (Pyrethroide) } \\
4209^{\star} \text { (PCP) }\end{array}$ \\
\hline Parathion (E 605) im Blut & $4209^{\star}$ \\
\hline p-Nitrophenol (E 605-Metabolit) im Urin & $4209^{\star}$ \\
\hline Pestizide I DDT, DDE, DDD & $3 \times 4209^{\star}$ \\
\hline Pestizide II Aldrin, Dieldrin im Blut & $2 \times 4209^{\star}$ \\
\hline Endrin im Blut & $4209^{\star}$ \\
\hline Heptachlor im Blut & $4209^{\star}$ \\
\hline Heptachlorepoxid im Blut & $4209^{\star}$ \\
\hline
\end{tabular}

Wichtiger Abrechnungshinweis (Gemäß Allgemeine Bestimmung Absatz 3. In M Laboratoriumsuntersuchungen)

3. Bei Weiterversand von Untersuchungsmaterial durch einen Arzt an einen anderen Arzt wegen der Durchführung von Laboruntersuchungen der Abschnitte M III und/oder M IV hat die Rechnungsstellung durch den Arzt zu erfolgen, der die Laborleistung selbst erbracht hat.

Spezialuntersuchungen von Schadstoffen können bei umweltmedizinischen Speziallaboratorien bestimmt werden.

\section{Vitalisierungskuren - Aufbau-Spritzen/Infusionen}

Infusions- oder Spritzen-Kuren mit z. B. Vitaminpräparaten werden in Praxen unterschiedlicher Fachgebiete sehr oft gewünscht, z. B. für

- Zustand n. Infekten, Erkrankungen, nach Operationen

- bei Stress, Müdigkeit, Leistungsabfall

- bei Tumorerkrankungen (begleitend)

Abrechnung mit ,graden“Eurobeträgen

- Pauschalbeträge sind nicht gestattet und so eine fehlerhaften Liquidationen müsste kein Patient bezahlen!

- Ärzte die „gerade und merkbare“ Honorarbeträge z. B. von 5,- oder 10,- usw. Euro anbieten möchten, sollten dies korrekt tun und auch dafür stets den „krummen“ Steigerungsfaktor angeben, der ein "gerades und merkbares" Honorar möglich macht, z. B.

\begin{tabular}{|l|l|r|r|r|}
\hline GOÄ Nr. & Kurzlegende & $\mathbf{1 f a c h ~ a ~}$ & $\begin{array}{r}\text { Steigerungs- } \\
\text { faktor }\end{array}$ & Endbetrag a \\
\hline $\mathbf{1}$ & Beratung & 4,66 & 2,15 & $\mathbf{1 0 , -}$ \\
\hline $\mathbf{3}$ & Eingehende Beratung & 8,74 & 2,29 & $\mathbf{2 0 , -}$ \\
\hline $\mathbf{8}$ & Ganzkörperuntersuchung & 15,15 & 2,24 & $\mathbf{3 4 , 0 0}$ \\
\hline $\mathbf{2 5 2}$ & i.m. Injektion & 2,33 & 2,15 & $\mathbf{5 , -}$ \\
\hline $\mathbf{2 5 3}$ & i.v. Injektion & 4,08 & 2,2 & $\mathbf{9 , -}$ \\
\hline $\mathbf{2 7 1}$ & i.v. Infusion bis 30 Min. & 6,99 & 2,146 & $\mathbf{1 5 , -}$ \\
\hline $\mathbf{2 7 3}$ & i.v. Infusion über 30 Min. & 10,49 & 2,0 & $\mathbf{2 1 , -}$ \\
\hline
\end{tabular}

Die Medikamente können nach GOÄ § 10 als Auslagen extra berechnet werden. 


\section{IGeL-Leistungen - zugeordnet den Fachgebieten}

\section{Vorbemerkung}

Nach angebotenen IGeL-Leistungen haben die Autoren recherchiert auf den Internetseiten der ärztlichen Fachverbände, bei KBV und Bundesärztekammer, sowie in den zahlreichen Praxis-Internetauftritten von Fachärzten und klinischen Abteilungen, ärztlichen Zeitschriften und zum Thema IGeL herausgegebenen Büchern.

Bei allen Abrechnungsbeispielen im Buch wurden die sinnvollen und möglichen - teilweise alternativen - Leistungen von Diagnostik und/oder Therapie mit entsprechenden GOÄ Nummern angegeben.

Die tabellarische Auflistung von GOÄ-Ziffern könnte dazu verleiten, mehrere Leistungen der Aufstellung zu erbringen und die GOÄ Nrn. einfach zu übernehmen... aber bitte berücksichtigen Sie, dass nach den Bestimmungen der GOÄ durchaus nicht alle angegeben Leistungspositionen bei einem Arzt-Patienten-Kontakt nebeneinander abrechenbar sind. Die Ausschlüsse aus den Allgemeinen Bestimmungen und die in den Anmerkungen zu den einzelnen GOÄ Nrn. gelten auch für analog angesetzte Leistungen.

Die von den Autoren gewählte Einteilung der IGeL-Leistungen nach Fachgebieten ist nur ein Korsett, um Leistungen aufzuführen; denn in der Regel werden viele dieser IGeL Leistungen von Ärzten unterschiedlicher Fachrichtungen mit entsprechenden Kenntnissen und Weiterbildungen erbracht.

Als Motivation und Ideenspende sind die angebotenen Leistungen der Ärzte (nach Recherche von den Autoren summiert) und Kliniken stets am Beginn eines Fachgruppenkapitel aufgeistet.

\section{ALLGEMEINMEDIZIN - INNERE MEDIZIN}

Was bietet die Fachgruppe der Allgemeinmediziner als IGeL-Leistungen an?

- Akupunktur

- Aufbaukuren

- Gesundheits-Check ausserhalb der 2-jährigen Krankenkassenregelung

- Herzinfarkt-Vorsorge

- HIV-Test

- Krebsvorsorgeuntersuchungen, zusätzliche

- Muskelaufbautraining

- Naturheilverfahren

- Osteoporose-Check

- Ostheopathie

- Prostatakrebs-Check

- Reisemedizin

- Sauerstofftherapie

- Schlaganfall-Risiko-Check

- Sporttauglichkeitsuntersuchung

- Vitaminen.Mangel, Abklärung von

- Vorsorge, erweiterte mit Tumormarkern, Risikoprofile

- Was bietet die Fachgruppe der Internisten an IGeL-Leistungen an?

- Antiaging-Beratung der Frau: Wann sind Hormongaben sinnvoll? (evtl mit Hormonstatus) - Wechseljahre auch des Mannes

- Braincheck (z. B. Hirnleistungstest)

- BURN-OUT"-Diagnostik. Aufbaukuren

- Fahrtauglichkeitsuntersuchungen

- Gefäß-Check

- Duplex Hals

- Doppler Beine

- Herz Check

- Echokardiographie

- Belastungs-EKG 
- Konfliktberatung (z. B. Ehekrisen,Stressbewältigung, Selbstfindung)

- Laboruntersuchungen z. B.

- Laktosetoleranztest (Milchzuckerverträglichkeit)

- Fruktosetoleranztest (Fruchtzuckerverträglichkeit)

- Laktulose-Test (Darmpassagezeit)

- HIV-TEST (AIDS-TEST)

- Blutgruppenbestimmung

- Sorbit-Toleranz-Test (Zuckeraustauschstoff-Verträglichkeit)

- Reisemedizin

- Risikokonstellation (genetisch oder erworben), Besprechung der persönl.

- Sporttauglichkeitsuntersuchunge

- Vorsorge, erweiterte (ohne Krankheitssymptome, ohne Beschwerden) plus Ruhe-EKG - plus Belastungs-EKG plus Ultraschall - plus Gastroskopie - plus Coloskopie

\section{Berufseignungsuntersuchungen}

\section{Private Berufseignungsuntersuchung}

Die Untersuchung nach dem Jugendarbeitsschutzgesetz ist strukturiert und die ärztlichen Leistungen sind für alle Bundesländer einheitlich. In der Regel wird die Untersuchung für öffentliche Kostenträger erbracht und kann in diesen Fällen nur mit dem 1fachen Gebührensatz berechnet werden.

Leider wird diese Untersuchung immer seltener, da viele Berufsanfänger zum Zeitpunkt des Eintritts ins Berufsleben das 18. Lebensjahr vollendet haben und damit nicht mehr dem Schutz des Jugendarbeitsschutzgesetzes unterliegen. Diese Versorgungslücke kann durch eine privatärztliche Berufseignungsuntersuchung als IGEL-Leistung - analoger Ansatz der GOÄ Nr. 32 - erbracht und berechnet werden. Da die Untersuchung in diesem Falle nicht für öffentliche Kostenträger erbracht wird, kann sie bis zum 2,3fachen Gebührensatz ohne Begründung und bis zum 3,5fachen Satz mit entsprechender Begründung berechnet werden.

\begin{tabular}{|c|c|c|c|}
\hline GOÄ Nr. & Kurzlegende & 1 fach $€$ & 2,3 fach $€$ \\
\hline 32 & $\begin{array}{l}\text { Untersuchung nach § } 32 \text { bis } 35 \text { und } 42 \text { des Jugendarbeitsschutz- } \\
\text { gesetzes } \\
\text { - Eingehende, das gewöhnliche Maß übersteigende Untersu- } \\
\text { chung - einschließlich: } \\
\text { - einfacher Seh-, Hör- und Farbsinnprüfung; } \\
\text { - Urinuntersuchung auf Eiweiß, Zucker und Erythrozyten; } \\
\text { - Beratung des Jugendlichen; } \\
\text { - schriftliche gutachterliche Äußerung; Mitteilung für die Perso- } \\
\text { nensorgeberechtigten; Bescheinigung für den Arbeitgeber } \\
\text { - bis zum 18. Lebensjahr }\end{array}$ & 23,31 & 53,62 \\
\hline $\begin{array}{l}32 \\
\text { analog }\end{array}$ & $\begin{array}{l}\text { Berufeignungsuntersuchung nach dem vollendeten } \\
\text { 18. Lebensjahr - Analoger Ansatz nach GOÄ Nr. } 32 \text { - GOÄ } \S 6 \text { (2) } \\
\text { GOÄ Originaltext siehe oben bei GOÄ Nr. } 32\end{array}$ & 23,31 & 53,62 \\
\hline
\end{tabular}

Sind ggf. weiterführende Untersuchungen erforderlich oder werden einzelne spezielle Untersuchungen vom jugendlichen Patienten gewünscht, so sind diese auch abrechenbar. Es empfiehlt sich aber eine schriftliche Vereinbarung mit dem Patienten.

Allgemeine berufliche Eignungsuntersuchungen

\begin{tabular}{|l|l|r|r|}
\hline GOÄ Nr. & Kurzlegende & $\mathbf{1 f a c h} €$ & $\begin{array}{r}{ }^{* 1,8 /} \\
\mathbf{2 , 3 f a c h} €\end{array}$ \\
\hline $\mathbf{3}$ & $\begin{array}{l}\text { Eingehende Beratung zur beruflichen Eignung (mind. 10 Min.)- } \\
\text { nicht neben Sonderleistungen }\end{array}$ & 8,74 & $\mathbf{2 0 , 1 1}$ \\
\hline $\mathbf{8}$ & Berufseignungsuntersuchung & 15,16 & $\mathbf{3 4 , 8 6}$ \\
\hline
\end{tabular}




\begin{tabular}{|c|c|c|c|}
\hline GOÄ Nr. & Kurzlegende & 1 fach $€$ &  \\
\hline 29 & $\begin{array}{l}\text { Gesundheitsuntersuchung eines Erwachsenen -nicht neben Be- } \\
\text { ratungen + Untersuchung }\end{array}$ & 25,65 & 59,00 \\
\hline $651^{*}$ & Ruhe EKG & 14,75 & 26,54 \\
\hline 652 & Belastungs-EKG & 25,94 & 59,66 \\
\hline $605^{*}$ & Lungenfunktionsprüfung & 14,11 & 25,39 \\
\hline 605a* & Darstellung der Flussvolumenkurve & 8,16 & 14,69 \\
\hline $5137^{*}$ & Brustorganübersicht & 26,23 & 47,21 \\
\hline \multirow[t]{2}{*}{70} & Kurze Bescheinigung & 2,33 & 5,36 \\
\hline & Labor & 1,0 & 1,15 \\
\hline $3560^{*}$ & Glukose & & \\
\hline $3511^{*}$ & Urin-Teststreifen & 2,91 & 3,35 \\
\hline $3531^{*}$ & Urinsediment & 4,08 & 4,69 \\
\hline
\end{tabular}

Bei Erwachsenen werden - abhängig vom gewählten Beruf - unterschiedliche Eignungsuntersuchungen gewünscht. Dies kann vom Herz-Kreislauf- und/oder Lungen-Check-up über Untersuchungen der Belastbarkeit der Wirbelsäule mit Röntgenaufnahmen bis zu allergologischen Tests gehen.

Allergologischer Berufseignungstest

Für einzelne Berufsgruppen ist das Risiko einer Allergie wesentlich höher als bei anderen. Zum Beispiel sind Bäcker, Friseure, Tankwarte, Mitarbeiter in Reinigungsbetrieben und der chemischen Industrie massiven Umwelteinflüssen ausgesetzt, während diese z. B. einen Angestellten im Verwaltungsdienst nicht betreffen.

\section{Beispiele aus der Praxis:}

- Ein 16jähriges Mädchen mit bekannter Nickelallergie möchte eine Friseurlehre absolvieren und vorher sicherstellen, dass sie nicht noch weitere Allergien hat.

\begin{tabular}{|l|l|r|r|}
\hline GOÄ Nr. & Kurzlegende & $\mathbf{1 f a c h} \boldsymbol{\text { f }}$ & $\begin{array}{r}\text { *1,8 } \\
\mathbf{2 , 3 f a c h} \boldsymbol{\text { }}\end{array}$ \\
\hline $\mathbf{1}$ & Beratung & 4,66 & $\mathbf{1 0 , 7 3}$ \\
\hline $\mathbf{5}$ & Untersuchung & 4,66 & $\mathbf{1 0 , 7 3}$ \\
\hline $\mathbf{3 8 0}$ & Epikutantest, je Test (1.-30. Test je Behandlungsfall) & 1,75 & $\mathbf{4 , 0 2}$ \\
\hline $\mathbf{3 8 5}$ & Pricktest, je Test (1.-20. Test je Behandlungsfall) & 2,62 & $\mathbf{6 , 0 3}$ \\
\hline $\mathbf{3 8 8}$ & $\begin{array}{l}\text { Reib-, Scratch- oder Skarifikationstest, je Test (bis zu 10 je Be- } \\
\text { handlungsfall) }\end{array}$ & 2,04 & $\mathbf{4 , 6 9}$ \\
\hline
\end{tabular}

Untersuchung für Arbeitnehmer im Bereich der Gastronomie nach Bundesseuchengesetz

\begin{tabular}{|c|c|c|c|}
\hline GOÄ Nr. & Kurzlegende & 1 fach $€$ & $\begin{array}{r}{ }^{*} 1,8 / \\
2,3 \text { fach } € \\
\end{array}$ \\
\hline 1 & Beratung & 4,66 & 10,72 \\
\hline 8 & Ganzkörperstatus & 15,15 & 34,86 \\
\hline 384 & Tuberkulinstempeltest & 2,33 & 5,36 \\
\hline \multirow[t]{2}{*}{70} & Kurze Bescheinigung & 2,33 & 5,36 \\
\hline & Labor & 1fach $€$ & 1,15 fach $€$ \\
\hline $4538^{\star}$ & Untersuchung z. Nachweis von Bakterien 1. Stuhluntersuchung & 7,00 & 8,04 \\
\hline $4538^{*}$ & 2. Stuhluntersuchung nach 3 Wochen* & 7,00 & 8,04 \\
\hline
\end{tabular}

Bei positivem Befund sind weitere Untersuchungen notwendig. 


\section{Untersuchungen gem. Fahrerlaubnis-Verordnung}

Die Änderung der Fahrerlaubnis-Verordnung (FeV) vom 1.1.1999 brachte neben der einheitlichen Einteilung der Fahrerlaubnisklassen in der EU auch regelmäßig wiederkehrenden Untersuchungen für die Inhaber bestimmter Fahrerlaubnisklassen:

- Ärztliche Untersuchung, gemäß Anlage $5 \mathrm{FeV}$ : Hier wird festgestellt, ob körperliche Erkrankungen vorliegen, die die Eignung zum Führen eines Kraftfahrzeuges beeinflussen können.

- Ärztliche Untersuchung des Sehvermögens, gemäß Anlage 6 FeV: Neben einer Bestimmung des Visus für die Ferne werden Farbensehen, räumliches Sehen und Gesichtsfeld getestet.

- Psychometrische Tests: Mit speziellen Testverfahren werden Belastbarkeit, Orientierungsleistung, Konzentrationsleistung, Aufmerksamkeitsleistung und Reaktionsfähigkeit überprüft.

Fahrtauglichkeitsuntersuchung

\begin{tabular}{|c|c|c|c|}
\hline GOÄ Nr. & Kurzlegende & 1 fach $€$ & 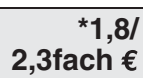 \\
\hline 1 & Beratung & 4,66 & 10,72 \\
\hline 8 & Ganzkörperstatus & 15,15 & 34,86 \\
\hline 70 & Kurze Bescheinigung & 2,33 & 5,36 \\
\hline \multirow[t]{2}{*}{$250^{*}$} & Blutentnahme & 2,33 & *4,20 \\
\hline & Labor & 1,0 & 1,15 \\
\hline $3511^{*}$ & Urin-Teststreifen & 2,91 & 3,35 \\
\hline $3531^{*}$ & Urinsediment & 4,08 & 4,69 \\
\hline $3560^{*}$ & Blutglukose & 2,33 & 2,68 \\
\hline $3585 \mathrm{H}^{*}$ * & Kreatinin & 2,33 & 2,68 \\
\hline $3592 \mathrm{H}^{*}$ * & GGT & 2,33 & 2,68 \\
\hline $3594 \mathrm{H}^{*}$ * & GOT & 2,33 & 2,68 \\
\hline $3595 \mathrm{H}^{*}$ * & GPT & 2,33 & 2,68 \\
\hline
\end{tabular}

Untersuchungen nach Fahrerlaubnis-Verordnung

\begin{tabular}{|l|l|l|l|}
\hline Fahrerlaubnis-Klasse & $\begin{array}{l}\text { Zeitpunkt der } \\
\text { Untersuchung }\end{array}$ & $\begin{array}{l}\text { körperliche und } \\
\text { Augenuntersuchung }\end{array}$ & Psychometrie \\
\hline C1, C1E & $\begin{array}{l}\text { beim Erstantrag und ab } \\
50 \text { alle 5 Jahre }\end{array}$ & ja & nein \\
\hline C, CE & $\begin{array}{l}\text { beim Erstantrag und dann } \\
\text { alle 5 Jahre }\end{array}$ & ja & nein \\
\hline D, D1, DE, D1E & $\begin{array}{l}\text { beim Erstantrag und dann } \\
\text { alle 5 Jahre }\end{array}$ & ja & $\begin{array}{l}\text { beim Erstantrag und } \\
\text { ab 50. Lebensjahr }\end{array}$ \\
\hline Fahrgastbeförderung & $\begin{array}{l}\text { beim Erstantrag und dann } \\
\text { alle 5 Jahre }\end{array}$ & ja & $\begin{array}{l}\text { beim Erstantrag und } \\
\text { ab 60. Lebensjahr }\end{array}$ \\
\hline
\end{tabular}

Gegenüberstellung der Fahrerlaubnisklassen vor 1999 und ab 2013

\begin{tabular}{|l|l|l|}
\hline $\begin{array}{l}\text { Fahrerlaubnis-l } \\
\text { Führerschein- } \\
\text { klasse vor 1999 }\end{array}$ & Betroffenes Fahrzeug & $\begin{array}{l}\text { Fahrerlaubnisklasse } \\
\text { ab 2013 }\end{array}$ \\
\hline $\mathbf{1}$ & Leistungsunbeschränkte Krafträder & A \\
\hline $\mathbf{1 a}$ & Krafträder bis $25 \mathrm{~kW}$, nicht mehr als 0,16 kW/kg & A2 \\
\hline $\mathbf{1 b}$ & $\begin{array}{l}\text { Krafträder bis } 125 \mathrm{~cm}^{3}, \text { bis } 11 \mathrm{~kW} \text { für 16- und 17jährige } \\
80 \mathrm{~km} / \mathrm{h} \text { bauartbedingte Höchstgeschwindigkeit }\end{array}$ & A1 \\
\hline $\mathbf{2}$ & Kfz über 7.500 $\mathrm{kg}$-Züge mit mehr als drei Achsen & $\mathbf{C}$ und CE \\
\hline
\end{tabular}




\begin{tabular}{|l|l|l|}
\hline $\begin{array}{l}\text { Fahrerlaubnis-/ } \\
\text { Führerschein- } \\
\text { klasse vor 1999 }\end{array}$ & Betroffenes Fahrzeug & $\begin{array}{l}\text { Fahrerlaubnisklasse } \\
\text { ab 2013 }\end{array}$ \\
\hline $\mathbf{3}$ & $\begin{array}{l}\text { Kfz bis 7.500 kg - Züge mit nicht mehr als 3 Achsen } \\
\text { (d. h. es kann ein einachsiger Anhänger mitgeführt wer- } \\
\text { den. Achsen mit einem Abstand von weniger als 1 m } \\
\text { voneinander gelten als eine Achse) }\end{array}$ & B, BE, C1 und C1E \\
\hline $\mathbf{2 , 3}$ & $\begin{array}{l}\text { Je nach dem zulässigen Gesamtgewicht des Fahr- } \\
\text { zeugs und Fahrerlaubnis zur Fahrgastbeförderung in } \\
\text { Kraftomnibussen }\end{array}$ & D, DE, D1 und D1E \\
\hline $\mathbf{4}$ & $\begin{array}{l}\text { Zweirädrige Kleinkrafträder und Fahrräder mit Hilfsmo- } \\
\text { tor bis } 50 \mathrm{~cm}^{3} / 50 \mathrm{~km} / \mathrm{h}\end{array}$ & AM \\
\hline
\end{tabular}

Seit 19.1.2013 gelten neue Bestimmungen im Fahrerlaubnisrecht. Sie resultieren aus der Umsetzung der 3. EU Führerscheinrichtlinie.

\section{Gültigkeitsdauer von Führerscheinen}

Die Gültigkeitsdauer aller ab dem 19.1.2013 ausgestellten Führerscheine wird auf 15 Jahre befristet (Ausnahme: Gültigkeitsdauer C und D - Klassen 5 Jahre). Es erfolgt eine Aktualisierung des Führerscheinmusters. Führerscheine enthalten dann ein Ausstellungsdatum und ein Ablaufdatum.

Die Einführung einer Gültigkeitsdauer für neue Führerscheine ermöglicht anlässlich der regelmäßigen Erneuerung die neuesten Maßnahmen zum Schutz gegen Fälschungen anzuwenden.

\section{Alte Führerscheine bleiben bis 2033 gültig}

Die alten Führerscheine müssen nicht umgetauscht werden und bleiben bis 2033 gültig. In den 27 Mitgliedstaaten der EU existieren derzeit 110 verschiedene Führerscheinmuster. Dies führt zu Transparenzproblemen für Bürger, Ordnungskräfte und Fahrerlaubnisbehörden und zur Fälschung von Dokumenten. Um dem entgegenzuwirken müssen spätestens bis zum 19.1.2033 alle vor dem 19.1.2013 ausgestellten Führerscheine umgetauscht werden. Bei der Verlängerung der Gültigkeit muss lediglich ein Passbild vorgelegt und die Verwaltungsgebühr gezahlt werden.

\section{Untersuchung über die gesundheitliche Eignung}

Die Untersuchung über die gesundheitliche Eignung kann von einem Arzt für Arbeits- oder Betriebsmedizin, einem Arzt bei einer Begutachtungsstelle für Fahreignung, einem Arzt des Gesundheitsamtes, einem anderen Arzt der öffentlichen Verwaltung oder von jedem Arzt für Allgemeinmedizin oder für Innere Medizin durchgeführt werden.

Die auf den folgenden Seiten abgebildete Bescheinigung (https://www.osnabrueck.de/images_design/Bescheinigung_ueber_die_aerztliche_Untersuchung.pdf) zeigt, welche Untersuchungsbefunde zu erheben sind.

Führerschein-Sehtest

\begin{tabular}{|l|l|r|r|}
\hline GOÄ Nr. & Kurzlegende & $\mathbf{1}$ fach $€$ & $\mathbf{2 , 3 f a c h} €$ \\
\hline $\mathbf{1}$ & Beratung & 4,66 & $\mathbf{1 0 , 7 3}$ \\
\hline $\mathbf{1 2 0 0}$ & Refraktionsbestimmung, subjektive & 3.44 & $\mathbf{7 , 9 1}$ \\
\hline $\mathbf{7 0}$ & Kurze Bescheinigung oder kurzes Zeugnis & 2,33 & $\mathbf{5 , 3 6}$ \\
\hline
\end{tabular}

Die Fahrerlaubnis-Verordnung (FeV) - Anlage 6 (zu §§ 12, 48 Abs. 4 und 5) schreibt vor, dass wenn der Bewerber den Sehtest nicht besteht, eine augenärztliche Untersuchung erforderlich ist. 


\section{Bescheinigung über die ärztliche Untersuchung}

von Bewerbern um die Erteilung oder Verlängerung einer Fahrerlaubnis der Klassen C, C1, CE, C1E, D, D1, DE, D1E oder der Fahrerlaubnis zur Fahrgastbeförderung für Taxen, Mietwagen, Krankenkraftwagen oder Personenkraftwagen im Linienverkehr oder bei gewerbsmäßigen Ausflugsfahrten oder Ferienziel-Reisen nach $\S 11$ Abs. 9 und $\S 48$ Abs. 4 und 5 der FahrerlaubnisVerordnung.

\section{Teil I (verbleibt beim Arzt)}

1. Personalien des Bewerbers

Familienname, Vorname

Tag der Geburt

Ort der Geburt

Wohnort

Straße/Hausnummer

2.

Hinweis für den untersuchenden Art:

Die Bescheinigung nach Teil II soll der Fahrerlaubnisbehörde vor Erteilung der Fahrerlaubnis Kenntnisse darüber verschaffen, ob bei dem Bewerber Beeinträchtigungen des körperlichen oder geistigen Leistungsvermögens vorliegen, die Bedenken gegen seine Eignung zum Führen von Kraftfahrzeugen begründen und gegebenenfalls Anlaß für eine weitergehende Untersuchungen vor Erteilung der Fahrerlaubnis geben.

Hierfür reicht in der Regel eine orientierende Untersuchung (sogenanntes "screening") der im folgenden genannten Bereiche aus; in Zweife Isfällen ist die Konsultation anderer Ärzte nicht ausgeschlossen.

3. Vorgeschichte

keine die Fahrfähigkeit einschränkende Krankheiten oder Unfälle durchgemacht

falls ja, welche:

4. Daten

Größe $(\mathrm{cm})$

Gewicht (kg)

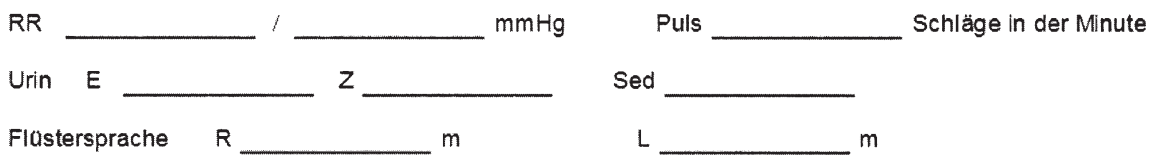

5. Allgemeiner Gesundheitszustand

$\square$ gut

$\square$ falls nicht ausreichend, nähere Erläuterung: 
E. Individuelle Gesundheitsleistungen von A - Z

6. Körperbehinderungen

$\square$ keine die Fahrfähigkeit einschränkende Behinderung

$\square$ falls ja, welche:

\section{Herz/Kreislauf}

keine Anzeichen fur Herz/Kreislaufstörungen

falls ja, welche:

8. Blut

keine Anzeichen einer schweren Bluterkrankung

falls ja, welche:

9. Erkrankungen der Niere

$\square$ keine Anzeichen einer schweren hsuffizienz

$\square$ falls ja, welche:

10. Endokrine Störungen

$\square$ keine Anzeichen einer Zuckerkrankheit

$\square$ Zuckerkrankheit - falls bekannt: mitohne hsulinbehandlung

$\square$ keine Anzeichen fur sonstige endokrine Störungen

$\square$ falls ja, welche:

\section{Nervensystem}

$\square$ keine Anzeichen fur Störungen

$\square$ falls ja, welche:

12. Psychische Erkrankungen/Sucht (Alkohol, Drogen, Arzneimittel)

keine Anzeichen einer Geistes- oder Suchterkrankung

falls ja, welche:

13. Gehör

keine Anzeichen fur eine schwere Störung des Hörvermögens

$\square$ falls ja, welche:

14. Erkrankungen mit erhöhter Tagesschläfrigkeit (z. B. Schlafstörungen)

keine Anzeichen fur Erkrankung mit erhöhter Tagesschläfrigkeit

$\square$ falls ja, welche: 


\section{Bescheinigung über die ärztliche Untersuchung}

von Bewerbern um die Erteilung oder Verlängerung einer Fahrerlaubnis der Klassen C, C1, CE, C1E, D, D1, DE, D1E oder der Fahrerlaubnis zur Fahrgastbeförderung für Taxen, Mietwagen, Krankenkraftwagen oder Personenkraftwagen im Linienverkehr oder bei gewerbsmäßigen Ausflugsfahrten oder Ferienziel-Reisen nach $\S 11$ Abs. 9 und $\S 48$ Abs. 4 und 5 der FahrerlaubnisVerordnung.

\section{Teil II (Kopie für den Arzt)}

Aufgrund der Angaben des Untersuchten

Familienname, Vorname

Tag der Geburt

Ort der Geburt

Wohnort

Straße/Hausnummer

und der von mir in dem nach Teil I vorgesehenen Umfang erhobenen Befunde empfehle ich vor Erteilung der Fahrerlaubnis

keine weitergehende Untersuchung, da keine Beeinträchtigungen des körperlichen oder geistigen Leistungsvermögens festgestellt werden konnten,

eine weitergehende Untersuchung wegen (Angabe der entsprechenden Befunde):

\section{Bescheinigung über die ärztliche Untersuchung}

von Bewerbern um eine Fahrerlaubnis der Klassen C, C1, CE, C1E, D, D1, DE, D1E oder der Fahrerlaubnis zur Fahrgast. beförderung für Taxen, Mietwagen, Krankenkraftwagen oder Personenkraftwagen im Linienverkehr oder bei gewerbsmäßigen Ausflugsfahrten oder Ferienziel-Reisen nach $\S 11$ Abs. 9 und $\S 48$ Abs. 4 und 5 der Fahrerlaubnis-Verordnung.

\section{Teil II (dem Bewerber auszuhändigen)}

\section{Aufgrund der Angaben des Untersuchten}

Familienname, Vorname

Tag der Geburt

Ort der Geburt

Wohnort

Straße/Hausnummer

und der von mir in dem nach Teil I vorgesehenen Umfang erhobenen Befunde empfehle ich vor Erteilung der Fahrerlaubnis

keine weitergehende Untersuchung, da keine Beeinträchtigungen des körperlichen oder geistigen Leistungsvermögens festgestellt werden konnten,

eine weitergehende Untersuchung wegen (Angabe der entsprechenden Befunde): 


\section{Präventive Untersuchungen Erwachsener}

Die Kassen der GKV, PKV und die Beihilfe zahlen ihren Versicherten alle zwei Jahre eine Gesundheitsuntersuchung (Check-up). Einige Patienten wünschen diese Leistung aber jährlich oder in einem kürzeren Abstand, wenn sich zum Beispiel gesundheitliche Beeinträchtigungen einstellen. Eine solche Zwischen-Untersuchung wird als Intervall-Check bezeichnet.

\section{Gesundheitsuntersuchungen und Intervall-Check können von allen Allgemeinmedizinern, Praktischen Ärzten und Internisten durchgeführt werden.}

Im Rahmen der GKV sind als Bestandteile der Gesundheitsuntersuchung nach der EBM Nr. 01732 ab dem 35. Lebensjahr vorgesehen:

- Anamnese

- Ganzkörperstatus

- Laboruntersuchungen

- Gesamtcholesterin

- Glukose

- Urinuntersuchung (Harnteststreifen) auf Eiweiß, Glukose, Erythrozyten, Leukozyten und Nitrit

- Beratung nach Abschluss der Untersuchung und Vorliegen der Laborergebnisse

- Folgerung aus den Ergebnissen

Werden Gesundheitsuntersuchungen vom GKV-Patienten vor dem 35. Lebensjahr bereits gewünscht oder in kürzeren Abständen als alle zwei Jahre, so sind diese Leistungen im Sinne von IGEL-Leistungen nach GOÄ abzurechnen und zwar in dem Umfang, in dem sie mit dem Patienten vereinbart und erbracht wurden.

Die Gesundheitsuntersuchung im Rahmen der PKV nach der GOÄ-Nr. 29 bezieht sich in der Legende nur auf

- Anamnese

- Ganzkörperstatus

- Beratung nach Abschluss der Untersuchung und Erörterung des Risikoprofils, Folgerung aus den Ergebnissen.

Alle erforderlichen ergänzenden Untersuchungen (ggf. EKG, Labor, Sono, Röntgen) sind möglich und gesondert zusätzlich abzurechnen.

Der einfache Gesundheits-Check nach EBM Nr. 01732 oder noch reduzierter nach GOÄ-Nr. 29 (die nur "Ganzkörperuntersuchung") sind zur Diagnostik von

- Herz- und Kreislauferkrankungen

- Nierenerkrankungen

- Stoffwechselstörungen

keineswegs ausreichend.

Die Gesundheitsuntersuchung nach GOÄ-Nr. 29 sollte mindestens um die in der GKV vorgeschriebenen Labor-Untersuchungen ergänzt werden:

Die „kleine“ Gesundheitsuntersuchung = kleiner Gesundheitscheck

\begin{tabular}{|c|c|c|c|}
\hline GOÄ Nr. & Kurzlegende & 1 fach $€$ & $\begin{array}{r}{ }^{*} 1,8 / \\
2,3 \mathrm{fach} \\
€\end{array}$ \\
\hline 29 & Gesundheitsuntersuchung eines Erwachsenen & 25,65 & 59,00 \\
\hline \multirow[t]{2}{*}{$250^{*}$} & Blutabnahme Vene & 2,33 & 5,36 \\
\hline & Labor & 1fach $€$ & 1,15 fach $€$ \\
\hline $3531^{*}$ & Urinsediment & 4,08 & 4,69 \\
\hline $3560^{*}$ & Glukose & 2,33 & 2,68 \\
\hline 3563.H1 & Gsamt-Cholesterin & 2,33 & 2,68 \\
\hline
\end{tabular}

Bis vor einigen Jahren gehörten zur Gesundheitsuntersuchung bei der GKV auch noch das RuheEKG und die Kreatininbestimmung. 
Zu einer sinnvollen Gesundheitsuntersuchung und auch zum Intervall-Check gehört die Überprüfung des Impfstatus des Patienten und damit auch die entsprechende Beratung und ggf. erforderliche Impfungen.

Neben einer Gesundheitsuntersuchung nach GOÄ-Nr. 29 sind am selben Tag Beratungen nach den GOÄ-Nrn. 1 und 3 nicht abrechenbar, aber wenn erforderlich natürlich an jedem anderen Tag.

Mit der Gesundheitsuntersuchung nach GOÄ-Nr. 29 lässt sich eine Krebsfrüherkennungs-untersuchung nach den GOÄ-Nrn. 27 (Frau) oder 28 (Mann) kombinieren.

Krebsvorsorge

\begin{tabular}{|c|c|c|c|}
\hline GOÄ Nr. & Kurzlegende & 1fach $€$ & 2,3 fach $€$ \\
\hline 27 & Krebsvorsorge bei einer Frau & 18,65 & 42,90 \\
\hline 28 & Krebsvorsorge bei einem Mann & 16,32 & 37,54 \\
\hline & Labor & 1 fach $€$ & 1,15 fach $€$ \\
\hline $3500^{*}$ & Stuhl auf Blut, 3mal & 5,25 & 6,03 \\
\hline
\end{tabular}

Die „große Gesundheitsuntersuchung“ = großer Gesundheitscheck

Zusätzliche (allgemeinmedizinische oder internistische) Untersuchungen über den Rahmen der skizzierten „kleinen“ Gesundheituntersuchung hinaus sind sinnvoll und abrechenbar, z. B. zum Ausschluss oder bei Verdacht auf Erkrankungen oder bei Kontrolluntersuchungen (bei entsprechenden Vorbefunden und im Rahmen von Tumornachsorge):

\begin{tabular}{|c|c|}
\hline Krankheit oder VD auf eine Erkrankung & Untersuchungsmethode \\
\hline $\begin{array}{l}\text { Koronare Herzerkrankung } \\
\text { Herz-Kreislauferkrankungen z. B. Hypertonie } \\
\text { Kontrolluntersuchungen }\end{array}$ & $\begin{array}{l}\text { EKG, Belastungs-EKG, } \\
\text { 24-Stunden EKG, } \\
\text { 24-Stunden-RRR-Messung }\end{array}$ \\
\hline $\begin{array}{l}\text { abdominelle Erkrankungen } \\
\text { gynäkologische Erkrankungen } \\
\text { Nierenerkrankungen } \\
\text { Prostata-Erkrankung } \\
\text { Schilddrüsen-Erkrankungen } \\
\text { Kontrolluntersuchungen }\end{array}$ & $\begin{array}{l}\text { Ultraschalluntersuchungen, } \\
\text { ggf. Radiologische Diagnostik, } \\
\text { Nuklearmedizinische Untersuchungen, } \\
\text { Laboruntersuchungen }\end{array}$ \\
\hline $\begin{array}{l}\text { Erkrankungen von Oesophagus, Magen/Duodenum } \\
\text { und Colon }\end{array}$ & $\begin{array}{l}\text { Endoskopie, } \\
\text { ggf. Ultraschall-Untersuchungen, } \\
\text { Radiologische Diagnostik, } \\
\text { Laboruntersuchungen }\end{array}$ \\
\hline $\begin{array}{l}\text { Gefäßerkrankungen } \\
\text { Kontrolluntersuchungen }\end{array}$ & Doppler-Untersuchung \\
\hline $\begin{array}{l}\text { Pulmonale Erkrankungen } \\
\text { Kontrolluntersuchungen }\end{array}$ & $\begin{array}{l}\text { Lungenfunktionsuntersuchungen, } \\
\text { Radiologische Diagnostik, } \\
\text { Laboruntersuchungen }\end{array}$ \\
\hline Erkrankungen des Skeletts & $\begin{array}{l}\text { Radiologische Diagnostik } \\
\text { Laboruntersuchungen }\end{array}$ \\
\hline $\begin{array}{l}\text { Stoffwechselstörungen } \\
\text { für zahlreiche Verdachts- oder Ausschlussdiagnosen } \\
\text { Kontrolluntersuchungen }\end{array}$ & Laboruntersuchungen \\
\hline
\end{tabular}

Große Gesundheitsuntersuchung

\begin{tabular}{|l|l|r|r|}
\hline GOÄ Nr. & Kurzlegende & $\mathbf{1 f a c h} €$ & $\begin{array}{r}\text { *1,8/ } \\
\mathbf{2 , 3 f a c h} €\end{array}$ \\
\hline $\mathbf{2 9}$ & Gesundheitsuntersuchung eines Erwachsenen & 25,65 & $\mathbf{5 9 , 0 0}$ \\
\hline $\mathbf{6 5 1 *}$ & EKG & 14,75 & $\mathbf{2 6 , 5 4}$ \\
\hline
\end{tabular}




\begin{tabular}{|c|c|c|c|}
\hline GOÄ Nr. & Kurzlegende & 1 fach $€$ & $\begin{array}{r}{ }^{*} 1,8 / \\
2,3 \text { fach } €\end{array}$ \\
\hline 652 & Belastungs-EKG & 25,94 & 59,66 \\
\hline $605^{*}$ & Lungenfunktionüberprüfung & 14,11 & 25,39 \\
\hline 410 & Ultraschalluntersuchung eines Organs & 11,66 & 26,81 \\
\hline 420 & Ultraschalluntersuchung von bis zu 3 weiteren Organen, je Organ & 4,66 & 10,72 \\
\hline 705 & Proktoskopie & 8,86 & 20,37 \\
\hline 690 & Rektoskopie & 20,40 & 46,92 \\
\hline \multirow[t]{2}{*}{$250^{*}$} & Blutabnahme Vene & 2,33 & 4,20 \\
\hline & Labor & 1 fach $€$ & 1,15 fach $€$ \\
\hline $3501^{*}$ & BKS & 3,50 & 4,03 \\
\hline $3531^{*}$ & Urinsediment & 4,08 & 4,69 \\
\hline 3563.H1* & Cholesterin & 2,33 & 2,68 \\
\hline 3565.H1* & Triglyceride & 2,33 & 2,68 \\
\hline 3583.H1* & Harnsäure & 2,33 & 2,68 \\
\hline 3585.H1* & Kreatinin & 2,33 & 2,68 \\
\hline 3587.H1* & Alkalische Phosphatase & 2,33 & 2,68 \\
\hline 3592.H1* & Gamma-GT & 2,33 & 2,68 \\
\hline 3595.H1* & GPT & 2,33 & 2,68 \\
\hline $3555^{\star}$ & Kalzium & 2,33 & 2,68 \\
\hline $3557^{*}$ & Kalium & 1,75 & 2.01 \\
\hline $3550^{*}$ & Blutbild & 3,50 & 4,03 \\
\hline $3551^{*}$ & Differentialblutbild & 1,17 & 1,35 \\
\hline
\end{tabular}

Intervall-Check: Die zusätzliche Gesundheitsuntersuchung

\section{Beispiele aus der Praxis:}

- Eine 36-jährige Patientin wünscht ein Jahr nach der letzten Gesundheitsuntersuchung nach GKVRichtlinien eine weitere Gesundheitsuntersuchung und möchte, dass das Angebot der Untersuchung um einen ausgedehnten Herzkreislauf-Check erweitert wird.

- Eine 62-jährige Frau wünscht mit der Bemerkung „Bei mir ist gesundheitlich irgendetwas nicht in Ordnung" eine zusätzliche Gesundheitsuntersuchung. Das Datum der letzten Gesundheitsuntersuchung liegt 8 Monate zurück.

- Eine 42-jährige Frau wünscht eine zusätzliche Gesundheitsuntersuchung, da sie -Raucherin - seit gut fünf Monaten unter Husten leide und sich schwach fühle. Eine klin. Untersuchung und eine Röntgen-Thorax-Untersuchung wegen dieser Beschwerden vor 3 Monaten war ohne path. Befunde. Das Datum der letzten Gesundheitsuntersuchung liegt 11 Monate zurück. Aus Angst vor einer Erkrankung will die Patientin eine Gesundheitsuntersuchung auf eigene Kosten.

\begin{tabular}{|l|l|r|r|}
\hline GOÄ Nr. & Kurzlegende & $\mathbf{1 f a c h} €$ & $\mathbf{2 , 3 f a c h} €$ \\
\hline $\mathbf{3}$ & $\begin{array}{l}\text { Eingehende Beratung (mind. 10 Min.) - nicht neben Sonderleis- } \\
\text { tungen }\end{array}$ & 8,74 & $\mathbf{2 0 , 1 1}$ \\
\hline $\mathbf{7}$ & $\begin{array}{l}\text { Untersuchung ein Organsystem: Haut, Stütz- u. Bewegungsorga- } \\
\text { ne, Brustorgane, Bauchorgane, weibl. Genitaltrakt - oder: }\end{array}$ & 9,33 & $\mathbf{2 1 , 4 5}$ \\
\hline $\mathbf{8}$ & Ganzkörperstatus & 15,15 & $\mathbf{3 4 , 8 6}$ \\
\hline $\mathbf{3 4}$ & Erörterung, mind. 20 Min. z. B. - nicht neben Nr. 3 & 17,49 & $\mathbf{4 0 , 2 2}$ \\
\hline
\end{tabular}

Anstatt den Intervall-Check als Gesundheitsuntersuchung nach GOÄ-Nr. $29(2,3$ fach $=59,00 €)$ abzurechnen, besteht auch die Möglichkeit nach z.B. GOÄ-Nrn.: $7+3$ oder $7+34$ oder $8+3$ oder $8+34$ abzurechnen. 


\section{Ergänzungsuntersuchungen zu Gesundheitsuntersuchung oder Intervall-Check}

Ergänzende Untersuchungen zum Intervall-Check up ergeben sich z. B. aus dem Wunsch des Patienten nach einer erweiterten Diagnostik oder aus schon erhobenen fraglichen Befunden.

\section{Gastro-Check}

\section{Beispiele aus der Praxis:}

- Ein 42-jähriger Gymnasiallehrer - z. Zt. ohne klinische Zeichen einer Erkrankung - möchte gerne einen jährlichen Gastro-Check, da er eine Ulcusanamnese hat.

- Eine 41-jährige verängstigte Krankenschwester hat in der Familienanamnese einen Fall von „Magen-Carc." (Mutter) und möchte jährliche Kontrollen.

\begin{tabular}{|c|c|c|c|}
\hline GOÄ Nr. & Kurzlegende & 1 fach $€$ & $\begin{array}{r}{ }^{*} 1,8 / \\
2,3 f a c h €\end{array}$ \\
\hline 3 & $\begin{array}{l}\text { Eingehende Beratung (mind. } 10 \text { Min.) - nicht neben Sonderleis- } \\
\text { tungen }\end{array}$ & 8,74 & 20,11 \\
\hline 7 & Untersuchung - Bauchorgane & 9,33 & 21,45 \\
\hline 682 & Gastroskopie - ggf. einschl. PE & 49,54 & 113,95 \\
\hline 683 & Gastroskopie einschl. Oesophaguskopie - ggf. einschl. PE & 58,29 & 134,06 \\
\hline 684 & $\begin{array}{l}\text { Bulbuskopie ggf. einschl. Oesophago- und Gastroskopie - ggf. } \\
\text { einschl. PE }\end{array}$ & 69,94 & 160,87 \\
\hline 685 & $\begin{array}{l}\text { Duodeno-/Jejunoskopie ggf. einschl. vorausgegangener Oeso- } \\
\text { phago-/Gastro/Bulboskopie - ggf. einschl. PE }\end{array}$ & 78,69 & 180,98 \\
\hline $250^{*}$ & Blutentnahme i.v. & 2,33 & 4,20 \\
\hline GOÄ Nr. & Labor & 1 fach $€$ & 1,15 fach $€$ \\
\hline 4234* & Helicobacter pylori - Antikörperschnelltest & 5,25 & 6,03 \\
\hline $3511^{*}$ & Helicobacter pylori - Urasetest im Biopsiematerial & 2,91 & 3,35 \\
\hline
\end{tabular}

Gefäß-Check der Extremitäten

\begin{tabular}{|l|l|r|r|}
\hline GOÄ Nr. & Kurzlegende & $\mathbf{1 f a c h} €$ & $\begin{array}{r}{ }^{*} \mathbf{1 , 8 /} \\
\mathbf{2 , 3 f a c h ~} \mathbf{€}\end{array}$ \\
\hline $\mathbf{3}$ & $\begin{array}{l}\text { Eingehende Beratung (mind. 10 Min.) - nicht neben Sonderleis- } \\
\text { tungen }\end{array}$ & 8,74 & $\mathbf{2 0 , 1 1}$ \\
\hline $\mathbf{6}$ & Untersuchung - Gefäßstatus & 5,83 & $\mathbf{1 3 , 4 1}$ \\
\hline $\mathbf{6 4 3}{ }^{*}$ & Peripherer Arterien- bzw. Venendruck und Strömungsmessung & 6,99 & $\mathbf{1 2 , 5 8}$ \\
\hline $\mathbf{6 4 4}^{*}$ & $\begin{array}{l}\text { Untersuchung der Strömungsverhältnisse in Extremitätenarte- } \\
\text { rien bzw. -venen, direktionale Ultraschall-Doppler-Technik }\end{array}$ & 10,49 & $\mathbf{1 8 , 8 9}$ \\
\hline
\end{tabular}

Gefäß-Check der hirnversorgenden Gefäße

Die Untersuchungen werden in der Regel auch von Nervenärzten und Neurologen mit entsprechender Qualifikation durchgeführt.

Beispiele aus der Praxis:

- Ein 57-jähriger Patient möchte aus Angst vor einem Schlaganfall - Vater hatte Caro-tisstenose eine Carotisstenose bei sich ausschließen.

- Eine 65-jährige Patientin möchte ihre hirnversorgenden Gefäße kontrollieren lassen. Bei einem Thorax-Röntgen wurden Aortenverkalkungen festgestellt.

\begin{tabular}{|l|l|r|r|}
\hline GOÄ Nr. & Kurzlegende & $\mathbf{1 f a c h} €$ & $\begin{array}{r}{ }^{* 1,8 /} \\
\mathbf{2 , 3 f a c h} €\end{array}$ \\
\hline $\mathbf{3}$ & $\begin{array}{l}\text { Eingehende Beratung (mind. 10 Min.) -nicht neben Sonderleis- } \\
\text { tungen }\end{array}$ & 8,74 & $\mathbf{2 0 , 1 1}$ \\
\hline $\mathbf{6}$ & Untersuchung - Gefässstatus & 5,83 & $\mathbf{1 3 , 4 1}$ \\
\hline
\end{tabular}




\begin{tabular}{|l|l|r|r|}
\hline GOÄ Nr. & Kurzlegende & $\mathbf{1 f a c h} €$ & $\begin{array}{r}{ }^{*} \mathbf{1 , 8 /} \\
\mathbf{2 , 3 f a c h} €\end{array}$ \\
\hline $\mathbf{6 4 5}$ & $\begin{array}{l}\text { Untersuchung Strömungsverhältnisse in hirnversorgenden Arte- } \\
\text { rien u. Periorbitalarterien mit direktionaler Ultraschall-Doppler- } \\
\text { Technik }\end{array}$ & 37,89 & $\mathbf{6 8 , 2 0}$ \\
\hline $\mathbf{4 1 0}$ & Sono eines Organs & 11,66 & $\mathbf{2 6 , 8 1}$ \\
\hline $\mathbf{4 2 0}$ & Sono bis zu 3 weiteren Organen, je Organ & 4,66 & $\mathbf{1 0 , 7 2}$ \\
\hline
\end{tabular}

\section{Abrechnungshinweis:}

Bei einer duplex-sonograhischen Untersuchung sind zusätzlich zur Leistung nach Nr. 645 die Nrn. 410 und 420 ansetzbar.

Wird eine Duplexuntersuchung im zweidimensionalen Bild farbcodiert durchgeführt, so kann ein höherer Steigerungsfaktor gewählt werden.

Thrombose-Risiko-Check

Bei familiärer Belastung kann heute vor Situationen, die mit einem erhöhten Thromboserisiko verbunden sind, durch ein Profil laborchemischer Untersuchungen von

- APC-Resistenz (Gerinnungstest)

- Protein-C-Aktivität

- Protein-S-Aktivität

- Antithrombin-III-Aktivität

- Lupus-Antikoagulans

- Homocystein

das Thromboserisiko untersucht werden.

Typische Situationen, in denen vermehrt Thrombosen auftreten, sind:

- die Einnahme von oralen Kontrazeptiva („Antibaby-Pille“)

- auch längere Flugreisen oder längere Busfahrten

können bei Patienten mit entsprechender Veranlagung zu Thrombosen und in Folge zu Lungenembolien u. ä. führen.

Beispiele aus der Praxis:

- Ein 40-jähriger Sportlehrer - ohne klinische Zeichen einer Erkrankung - möchte gerne an einem Marathonlauf teilnehmen und wünscht zuvor ein Belastungs-EKG.

- Da der Vater mit 72 Jahren einen Schlaganfall erlitt, möchte der 41-jährige Sohn eine Untersuchung zur Herzinfarkt und Schlaganfall-Prävention.

- 42-jährige Patientin - intensive Tennisspielerin - wünscht Belastungs-EKG zur Feststellung der Leistungsfähigkeit. Zusätzlich wird eine Spirometrie gewünscht.

- 62-jährige Patientin mit Herzinsuffizienz (nach NYHA-Klassifikation als II klassifiziert) wünscht auch ohne Verschlechterung des Zustandes kurzfristige Kontrolluntersuchungen.

Herz-Kreislauf-Check

\begin{tabular}{|c|c|c|c|}
\hline GOÄ Nr. & Kurzlegende & 1 fach $€$ & $\begin{array}{r}{ }^{*} 1,8 / \\
2,3 f a c h \\
\end{array}$ \\
\hline 3 & $\begin{array}{l}\text { Eingehende Beratung (mind. } 10 \text { Min.) - nicht neben Sonderleis- } \\
\text { tungen }\end{array}$ & 8,74 & 20,11 \\
\hline 8 & Ganzkörperstatus & 15,15 & 34,86 \\
\hline $651^{*}$ & Ruhe EKG & 14,75 & 26,55 \\
\hline 652 & Belastungs-EKG & 25,94 & 59,66 \\
\hline \multirow[t]{2}{*}{$250^{*}$} & Blutentnahme aus Vene & 2,33 & 4,20 \\
\hline & Labor & 1fach $€$ & *1,15fach $€$ \\
\hline 3562.H1* & Gesamt-Cholesterin & 2,33 & 2,68 \\
\hline 3563. H1* & HDL-Cholesterin & 2,33 & 2,68 \\
\hline 3564.H1* & LDL-Cholesterin & 2,33 & 2,68 \\
\hline
\end{tabular}




\begin{tabular}{|c|c|c|c|}
\hline & Labor & 1 fach $€$ & *1,15fach $€$ \\
\hline 3565.H1* & Triglyceride & 2,33 & 2,68 \\
\hline $3560^{*}$ & Glukose & 2,33 & 2,68 \\
\hline $4084^{*}$ & Homocystein - analoger Ansatz & 33,22 & 38,21 \\
\hline 3933* & Fibrinogen & 5,83 & 6,70 \\
\hline $3730^{*}$ & Lipoprotein (a) & 17,49 & 20,11 \\
\hline $3741^{*}$ & CRP (Ligandenassay) & 11,66 & 13,41 \\
\hline
\end{tabular}

Weiterführende Untersuchungen zu Herzinfarkt- und Schlaganfall-Prävention

- Koronararteriographie, nicht-invasive, z. B. mit Elektronenstrahl-Tomographie (EBCT) oder mit Mehrschicht-Computer-Tomographie, auch mit Bestimmung von Koronarkalk

- Schlaganfall-Vorsorge: Doppler-Sonographie der hirnversorgenden Gefäße bei fehlenden anamnestischen oder klinischen Auffälligkeiten („Stroke-Check“)

\section{Leber-Check}

Beispiele aus der Praxis:

- Ein 54-jähriger Mann - ohne klinische Zeichen einer Erkrankung - möchte gern einen Sonocheck der Leber und entsprechende Laboruntersuchungen, da er viel Alkohol konsumiert.

\begin{tabular}{|c|c|c|c|}
\hline GOÄ Nr. & Kurzlegende & 1 fach $€$ & $\begin{array}{r}{ }^{*} 1,8 / \\
2,3 f a c h \\
\end{array}$ \\
\hline 3 & $\begin{array}{l}\text { Eingehende Beratung (mind. } 10 \text { Min.) - nicht neben Sonderleis- } \\
\text { tungen }\end{array}$ & 8,74 & 20,11 \\
\hline 7 & Untersuchung Bauchorgane & 15,15 & 34,86 \\
\hline 410 & Sonographie Leber & 11,66 & 26,81 \\
\hline 420 & Sonographie Gallenblase & 4,66 & 10,73 \\
\hline 420 & Sonographie große abd. Gefässe & 4,66 & 10,73 \\
\hline 420 & Sonographie ggf. 1 weiteres Organ & 4,66 & 10,73 \\
\hline \multirow[t]{2}{*}{$250^{*}$} & Blutentnahme & 2,33 & 4,20 \\
\hline & Labor: Leberprofil & 1 fach $€$ & ${ }^{\star} 1,15 \mathrm{fach} €$ \\
\hline 3587.H1* & Alkalische Phosphatase & 2,33 & 2,68 \\
\hline 3581.H1* & Bilirubin gesamt & 2,33 & 2,68 \\
\hline $3574^{*}$ & Serumelektrophorese & 11,66 & 13,41 \\
\hline 3573.H1* & Gesamteiweiss & 1,75 & 2.01 \\
\hline 3592.H1* & Gamma-GT & 2,33 & 2,68 \\
\hline 3593.H1* & GLDH & 2,91 & 3,35 \\
\hline 3594.H1* & GOT & 2,33 & 2,68 \\
\hline 3595.H1* & GPT & 2,33 & 2,68 \\
\hline
\end{tabular}

\section{Lungenfunktions-Check}

Beispiele aus der Praxis:

- Eine 46-jährige Frau - ohne klinische Zeichen einer Erkrankung - möchte gern einen Lungenfunktions-Check, da sie seit 22 Jahren raucht und sich angeblich in letzter Zeit beim Tennisspielen häufiger „Atemnot“ einstelle.

- 59-jähriger fanatischer Taucher möchte sich über die Leistungsfähigkeit seiner Lungen informieren. Er wünscht ferner ein Belastungs-EKG.

- 39-jähriger Raucher seit dem 16. Lebensjahr - ohne klinische Zeichen einer Erkrankung wünscht einen Lungenfunktions-Check mit Belastungs-EKG. 


\begin{tabular}{|c|c|c|c|}
\hline GOÄ Nr. & Kurzlegende & 1 fach $€$ & $\begin{array}{r}{ }^{*} 1,8 / \\
2,3 \text { fach } €\end{array}$ \\
\hline 3 & $\begin{array}{l}\text { Eingehende Beratung (mind. } 10 \text { Min.) - nicht neben Sonderleis- } \\
\text { tungen }\end{array}$ & 8,74 & 20,11 \\
\hline 7 & Untersuchung Bauchorgane & 15,15 & 34,86 \\
\hline $605^{*}$ & Ruhespirographische Untersuchung & 14,11 & 25,39 \\
\hline $605 a^{*}$ & Darstellung der Flußvolumenkurve & 8,16 & 14,69 \\
\hline 608* & Ruhespirographische Teil-Untersuchung & 4,43 & 7,97 \\
\hline $610^{*}$ & Ganzkörperplethysmographie & 35,26 & 63,48 \\
\hline $5137^{*}$ & Brustorganübersicht & 26,23 & 47,21 \\
\hline $505^{*}$ & Atmungsunterweisung - analoger Ansatz der Nr. 505 & 4,95 & 12,38 \\
\hline
\end{tabular}

\section{Stoffwechsel-Check}

\section{Beispiele aus der Praxis:}

- Eine 36-jährige Patientin mit Übergewicht möchte jährliche Diabetesrisiko- und LipidstoffwechselChecks und daneben zusätzlich einen Herz-Kreislauf-Check.

- 48-jähriger Patient mit massivem Übergewicht möchte quartalsmäßig Beratung zur Gewichtsreduktion und entsprechende Untersuchungen.

- Eine 42-jährige Patientin (ohne BZ-Erhöhungen bei den bisherigen Laboruntersuchungen), deren Eltern beide an Diabetes Typ-2 leiden, möchte halbjährliche Diabetes- und LipidstoffwechselChecks.

Individuelle Diätberatung - Beratung zur Gewichtsreduktion - Diät-Beratung ohne Vorliegen einer Erkrankung - Gruppenbehandlung bei Adipositas

\begin{tabular}{|c|c|c|c|}
\hline GOÄ Nr. & Kurzlegende & 1 fach $€$ & $\begin{array}{r}{ }^{*} 1,8 / \\
2,3 f a c h €\end{array}$ \\
\hline 3 & $\begin{array}{l}\text { Eingehende Beratung (mind. } 10 \text { Min.) - nicht neben Sonderleis- } \\
\text { tungen }\end{array}$ & 8,74 & 20,11 \\
\hline 1 & Beratung & 4,66 & 10,72 \\
\hline 8 & Ganzkörperuntersuchung & 15,15 & 34,85 \\
\hline 33 & $\begin{array}{l}\text { Diätschulung - analog GOÄ § } 6 \text { (2) } \\
\text { GOÄ Kurztext Nr. 33: Strukturierte Schulung einer Einzelperson } \\
\text { mit einer Mindestdauer von } 20 \text { Minuten ... }\end{array}$ & 17,49 & 40,22 \\
\hline $\begin{array}{l}34 \\
\text { analog }\end{array}$ & $\begin{array}{l}\text { Ausgedehnte Diät/Erörterung ohne Vorliegen einer Erkrankung } \\
\text { - mind. } 20 \text { Min. - analog GOÄ § } \mathbf{6} \text { (2) } \\
\text { GOÄ Kurztext Nr. 34: Erörterung (Dauer mindestens } 20 \text { Minuten) der Aus- } \\
\text { wirkungen einer Krankheit auf die Lebensgestaltung... }\end{array}$ & 300 & 40,22 \\
\hline 20 & $\begin{array}{l}\text { Gruppenbehandlung bei Adipositas } \\
\text { (4-12 Teilnehmer, mind. } 50 \text { Minuten) - analog GOÄ } \S 6 \text { (2) } \\
\text { GOÄ Kurztext Nr. 20: Beratungsgespräch in Gruppen von } 4 \text { bis } 12 \text { Teilneh- } \\
\text { mern im Rahmen der Behandlung von chronischen Krankheiten ... }\end{array}$ & 120 & 16,09 \\
\hline 76 & Schriftlicher Diätplan & 4,08 & 9,34 \\
\hline 77 & Kurplanung & 8,74 & 20,10 \\
\hline $250^{*}$ & Blutentnahme & 2,33 & 4,20 \\
\hline
\end{tabular}

Labor: Diabetes-Risiko-Check

\begin{tabular}{|c|c|c|c|c|}
\hline GOÄ Nr. & Kurzlegende & Labor & 1fach € & 1,15 fach $€$ \\
\hline $3560^{*}$ & Blutzucker & & 2,33 & 2,68 \\
\hline $3561^{*}$ & HBA1c & & 11,66 & 13,41 \\
\hline $3613^{*}$ & Blutzuckerbelastung & & 9,33 & 10,73 \\
\hline $3652^{*}$ & Urin-Streifentest & & 2,04 & 2,35 \\
\hline
\end{tabular}


Lipid- und Gicht-Check

\begin{tabular}{|c|c|c|c|c|}
\hline GOÄ Nr. & Kurzlegende & Labor & 1 fach $€$ & ${ }^{*} 1,15$ fach $€$ \\
\hline $3560^{*}$ & Blutzucker & & 2,33 & 2,68 \\
\hline $3562 \mathrm{H}^{*}$ * & Cholesterin & & 2,33 & 2,68 \\
\hline $3563 \mathrm{H} 1^{*}$ & HDL & & 2,33 & 2,68 \\
\hline $3564 \mathrm{H}^{*}$ * & LDL & & 2,33 & 2,68 \\
\hline $3565 \mathrm{H}^{*}$ * & Triglyceride & & 2,33 & 2,68 \\
\hline $3584 \mathrm{H}^{*}$ * & Harnstoff & & 2,33 & 2,68 \\
\hline $3583 \mathrm{H} 1$ * & Harnsäure & & 2,33 & 2,68 \\
\hline $3652^{*}$ & Urin-Streifentest & & 2,04 & 2,35 \\
\hline
\end{tabular}

\section{Sono Check verschiedener Organe}

Bei einem umfassenden oder nur teilweisen sonographischen Check-up der inneren Organe etc. handelt es sich meist um gewünschte Zusatzleistungen zu Gesundheitsuntersuchung, Intervalloder General Check-up oder einfach um den akuten Patientenwunsch, sich z. B. über seine „Leber“ oder „Niere“ zu informieren. In der Regel sind zusätzlich zum Sono-Organ-Check noch Beratungsleistungen nach Nr. 1 oder 3, Untersuchungen und entsprechende Laborparameter erforderlich.

\section{Beispiele aus der Praxis:}

- Ein 55-jäh. Patient mit leicht erhöhter Gamma-GZ wünscht einen Sono-Lebercheck.

- Ein 58-jähriger Patient mit bekannten Gallenstein wünscht häufige Sono-Kontrollen.

- Eine 24-jährige Patientin mit chron. rezidivierendem Harnwegsinfekt wünscht häufige SonoChecks ihrer Nieren.

- Eine 38-jäh. Patientin mit bekannten Nierensteinen wünscht häufige Sono-Kontrollen.

- Eine 18-jährige Patientin hält ihren Hals für „zu dick“ und möchte eine Sono ihrer Schilddrüse.

\section{Sono-Check der abdominellen Organe und Gefäße}

Beim sonographischen Check-up (zu Teilen Bestanteil von Intervall-, Facharzt- oder General Check-

up) der inneren Organe geht es im abdominellen Bereich um die Darstellung

- der Leber

- der Gallenblase und der Gallenwege

- der Bauchspeicheldrüse

- der Milz

- der Nieren

- der großen Oberbauchgefäße Vena cava, Aorta etc.

- und die darstellbaren Bezirke im Bereich des Magens und des Darmes.

\begin{tabular}{|l|l|r|r|}
\hline GOÄ Nr. & Kurzlegende & $\mathbf{1 f a c h} €$ & $\mathbf{2 , 3 f a c h} €$ \\
\hline $\mathbf{4 1 0}$ & ein Organ & 11,66 & $\mathbf{2 6 , 8 1}$ \\
\hline $\mathbf{4 2 0}$ & Sonographie bis zu 3 weiteren Organen, je Organ & 4,66 & $\mathbf{1 0 , 7 3}$ \\
\hline
\end{tabular}

Sono-Check des Urogenitaltraktes mit zusätzlichen transkavitären Untersuchungen

\begin{tabular}{|l|l|r|r|}
\hline GOÄ Nr. & Kurzlegende & $\mathbf{1 f a c h} €$ & $\begin{array}{r}{ }^{*} \mathbf{1 , 8 /} \\
\mathbf{2 , 3 f a c h} €\end{array}$ \\
\hline $\mathbf{4 1 0}$ & ein Organ & 11,66 & $\mathbf{2 6 , 8 1}$ \\
\hline $\mathbf{4 0 3}{ }^{*}$ & Zuschlag bei transkavitärer Untersuchung (rektal/vaginal) & 8,74 & $\mathbf{1 5 , 7 4}$ \\
\hline $\mathbf{4 2 0}$ & Sonographie bis zu 3 weiteren Organen, je Organ & 4,66 & $\mathbf{1 0 , 7 3}$ \\
\hline
\end{tabular}

Sono-Check der Schilddrüse + ggf. Halsgefäße

\begin{tabular}{|l|l|r|r|}
\hline GOÄ Nr. & Kurzlegende & $\mathbf{1 f a c h} €$ & $\mathbf{2 , 3 f a c h} €$ \\
\hline $\mathbf{4 1 7}$ & Sonographie der Schilddrüse & 12,24 & $\mathbf{2 8 , 1 5}$ \\
\hline $\mathbf{4 2 0}$ & Sonographie bis zu 3 weiteren Organen, je Organ & 4,66 & $\mathbf{1 0 , 7 3}$ \\
\hline
\end{tabular}




\section{E. Individuelle Gesundheitsleistungen von A - Z}

\section{Sono-Nieren-Check + Labor}

\section{Beispiele aus der Praxis:}

- 68-jähriger Patient Patient mit Nierencysten wünsch jährliche Kontrollen der Cysten auf Größe und Anzahl sowie entsprechende Laborparameter.

- 58-jährige Patienten mit bekannter Hyperurikämie wünscht jährlichen Nieren-Check.

\begin{tabular}{|c|c|c|c|}
\hline GOÄ Nr. & Kurzlegende & 1 fach $€$ & $\begin{array}{r}{ }^{*} 1,8 / \\
2,3 f a c h\end{array}$ \\
\hline 410 & Sonographie rechte Niere & 11,66 & 26,81 \\
\hline 420 & Sonographie linke Niere & 4,66 & 10,73 \\
\hline 420 & Sonographie grosse abd. Gefässe & 4,66 & 10,73 \\
\hline 420 & Sonographie ggf. 1 weiteres Organ; Blase & 4,66 & 10,73 \\
\hline \multirow[t]{2}{*}{$250^{*}$} & Blutentnahme & 2,33 & 4,20 \\
\hline & Labor & 1 fach $€$ & *1,15fach $€$ \\
\hline 3550* & Blutbild & 3,50 & 4,03 \\
\hline 3583.H1 & Harnsäure & 2,33 & 2,68 \\
\hline 3584.H1 & Harnstoff & 2,33 & 2,68 \\
\hline 3585.H1 & Kreatinin & 2,33 & 2,68 \\
\hline 3557 & Kalium & 1,75 & 2,01 \\
\hline 3558 & Natrium & 1,75 & 2,01 \\
\hline $3511^{\star *}$ & Urin-Teststreifen & 2,91 & 3,35 \\
\hline
\end{tabular}

\section{Sono Schilddrüsen-Check + Labor}

Besonders bei Frauen ab dem 35. Lebensjahr sollte zum Ausschluss einer Unterfunktion der Schilddrüse eine TSH-Bestimmung erfolgen.

\section{Beispiele aus der Praxis:}

- Eine 33-jährige Patientin hält ihren Hals für „zu dick“ und möchte einen Sono-Check ihrer Schilddrüse

- Ein 42-jähriger Patienten - ohne klinische Zeichen einer Erkrankung - möchte eine Untersuchung zum Ausschluss einer Schilddrüsenüberfunktion.

\begin{tabular}{|c|c|c|c|}
\hline GOÄ Nr. & Kurzlegende & 1fach € & $\begin{array}{r}{ }^{*} 1,8 / \\
2,3 \text { fach } €\end{array}$ \\
\hline 417 & Sonographie der Schilddrüse & 12,24 & 28,15 \\
\hline $250^{*}$ & Blutentnahme aus Vene & 2,33 & 4,20 \\
\hline & Labor & 1 fach $€$ & ${ }^{\star} 1,15 \mathrm{fach} €$ \\
\hline 4022.H4* & T3 & 14,57 & 16,76 \\
\hline 4023.H4* & T4 & 14,57 & 16,76 \\
\hline 4030.H4* & TSH & 14,57 & 16,76 \\
\hline
\end{tabular}

Die umfassende Gesundheitsuntersuchung: General-Check-up/Manager-Check

General-Check-up ist die Umschreibung für die umfassendste ambulante Vorsorge-Untersuchung, einschl. Sonographie, Lungenfunktion, Endoskopie, Belastungs-EKG, ausgedehnter Laboruntersuchungen, Röntgen usw. und entsprechender Beratungen, besonders zu sportlicher Bewegung und gesunder Ernährung.

Der General-Check-up wird seit Jahren z. B. von der Deutschen Klinik für Diagnostik in Wiesbaden durchgeführt. Das Konzept stammt von der Mayo-Klinik in den USA. Dort und später auch bei uns wurde es zuerst für zahlungskräftige Patienten und danach auch von Firmen zur Untersuchung ihrer leitenden Angestellten eingeführt und hat deshalb in der Bevölkerung den landläufigen Namen Manager-Check-up erhalten. In Wiesbaden werden die Patienten mehreren Fachärzten vorgestellt, damit eine umfassende Untersuchung durchgeführt werden kann. 
Während die Gesundheitsuntersuchung - auch mit zusätzlichen Leistungen - in der Regel eine Leistung von Allgemeinmedizinern oder Internisten darstellt, ist ein General-Check-up oder Manager-Check immer eine Gemeinschaftsleistung mehrerer Ärzte unterschiedlicher Fachgruppen. Für Untersuchungen, die nur ein Arzt durchführt, kann der Begriff seriöserweise nicht verwendet werden.

Das Wirtschaftsmagazin Capital (9/2003) sah schon vor 10 Jahren die Zukunft im Check-up und schrieb ... „Die Vorsorgebranche boomt. Die Zahl der Kliniken, die entsprechende Untersuchungen anbieten, steigt entsprechend. Die gesetzlichen Kassen zahlen für solche Check-ups aber nicht. Bei den privaten hängt es vom Verhandlungsgeschick des Versicherten ab. Im Unterschied zur Vorbeugung beim Hausarzt sind die Untersuchungen beim Spezialisten umfassender - und deren Ablauf ist besser organisiert ...".

Auch die niedergelassenen Vertragsärzte könnten einen umfassenden General-Check anbieten. Wenn sie verschiedene Fachkollegen aus Praxis und Klinik in der Umgebung in dieses Angebot einbeziehen, so ist der General-Check für die Patienten umso glaubwürdiger. Hierzu sind Absprachen unter den Kollegen und festgelegte Untersuchungs-Programme zum Nutzen von Patient und Vertragsärzten notwendig. Vor allem aber müssen Vertragsärzte die Angst aufgeben, Patienten ggf. in eine Chefarztambulanz zu schicken. Viele Manager fühlen sich für den großen Check-up besser in einer Klinkambulanz aufgehoben als in einer Praxis.

Eine gute Zusammenarbeit zwischen Praxis und Klinik mit einem umfangreichen diagnostischen Angebot kann hier Verbesserungen - d. h. den Patienten zurück zu seinem Hausarzt - bringen. Primär wird der Hausarzt oder Internist beim General-Check-up als „guter Verteiler“ an seine Fachkollegen gesehen, als „Sammler“ und Berater der erhobenen Befunde.

Eine Standardisierung der Leistungen eines General-Checks gibt es nicht. Der Leistungsumfang ist abhängig von

- den Wünschen des Patienten

- von den Kenntnissen des primär behandelnden Arztes

- von der Einstellung des Arztes zu Fachkollegen zu überweisen

- von den Kenntnissen der kooperierenden Fachkollegen des Arztes.

Vergleich von Inhalt/Umfang verschiedener umfassender Vorsorge-Untersuchungen

- Wichtig: Nicht alle aufgeführten Untersuchungen sind bei einem Arzt-/Patientenkontakt abrechenbar.

\begin{tabular}{|c|c|c|c|c|c|}
\hline Ärztliche Leistungen & $\begin{array}{l}\text { General- } \\
\text { Check } \\
\text { (IGEL) }\end{array}$ & DKD** & $\begin{array}{l}\text { Mayo- } \\
\text { Clinic }^{\star \star *}\end{array}$ & GOÄ Nrn. & $\begin{array}{l}{ }^{*} 1,8 / 2,3- \\
\text { fach } €\end{array}$ \\
\hline \multicolumn{6}{|l|}{ 1. Klinische Untersuchungen } \\
\hline $\begin{array}{l}\text { Eingehende Anamnese und Internis- } \\
\text { tische Ganzkörperuntersuchung }\end{array}$ & $\checkmark$ & $\checkmark$ & $\checkmark$ & $\begin{array}{c}29 \\
\text { alternativ } \\
8\end{array}$ & $\begin{array}{l}58,99 \\
15,15\end{array}$ \\
\hline Überprüfung des Impfstatus & $\checkmark$ & $?$ & $?$ & 3 & 20,11 \\
\hline Hörprüfung & fakultativ & $\checkmark$ & fakultativ & 1400 & 10,19 \\
\hline Augenuntersuchung & fakultativ & $\checkmark$ & fakultativ & & \\
\hline $\begin{array}{l}\text { Gynäkologische Vorsorge-Untersu- } \\
\text { chung }\end{array}$ & fakultativ & $\checkmark$ & fakultativ & 27 & 42,90 \\
\hline \multicolumn{6}{|c|}{ 2. Apparative-diagnostische Untersuchungen } \\
\hline EKG und Belastungs-EKG & $\checkmark$ & $\checkmark$ & $\begin{array}{l}\text { ab } 40 \mathrm{~J} . \\
\text { Ruhe-EKG }\end{array}$ & $\begin{array}{l}651^{*} \\
652\end{array}$ & $\begin{array}{l}26,55 \\
59,66\end{array}$ \\
\hline Langzeit-EKG & & & & $659^{*}$ & 41,97 \\
\hline Langzeit-Blutdruckmessung & & & & $654^{*}$ & 15,74 \\
\hline Lungenfunktionsprüfung & $\checkmark$ & $\checkmark$ & $\begin{array}{c}\text { bei } \\
\text { Rauchern }\end{array}$ & $\begin{array}{l}605^{*} \\
605 a\end{array}$ & $\begin{array}{l}25,39 \\
14,69\end{array}$ \\
\hline
\end{tabular}


E. Individuelle Gesundheitsleistungen von A - Z

\begin{tabular}{|c|c|c|c|c|c|}
\hline Ärztliche Leistungen & $\begin{array}{l}\text { General- } \\
\text { Check } \\
\text { (IGEL) }\end{array}$ & $D_{K} D^{* *}$ & $\begin{array}{l}\text { Mayo- } \\
\text { Clinic**** }\end{array}$ & GOÄ Nrn. & $\begin{array}{l}\text { *1,8/2,3- } \\
\text { fach } €\end{array}$ \\
\hline \multicolumn{6}{|l|}{ 3. Endoskopische Untersuchungen } \\
\hline - Rektoskopie & fakultativ & ab $50 \mathrm{~J}$. & fakultativ & 690 & 46,92 \\
\hline - Sigmoidoskopie & & & & 689 & 93,83 \\
\hline - Gastroskopie & fakultativ & - & - & 682 & 113,95 \\
\hline - Coloskopie & & & & 687 & 201,09 \\
\hline \multicolumn{6}{|c|}{ 4. Ultraschall-Untersuchungen/Doppler } \\
\hline Sono Innere Organe - ein Organ & $\checkmark$ & $\checkmark$ & fakultativ & 410 & 26,81 \\
\hline - weitere bis zu 3 Organe je Organ & $\checkmark$ & $\checkmark$ & fakultativ & 420 & 10,73 \\
\hline - Schilddrüse & & & & 417 & 28,15 \\
\hline - Gynäkologische Sonographie- & & & & 410 & 26,81 \\
\hline weitere bis zu 3 Organe, je Organ & & & & 420 & 10,73 \\
\hline - Transvaginale Sonographie & & & & 410 & 26,81 \\
\hline - Transrektale Sonographie Prostata & fakultativ & - & - & 410 & 26,81 \\
\hline - Zuschlag bei transkavitärer Sono & fakultativ & - & - & $403^{*}$ & $15,74^{\star}$ \\
\hline - Doppler-Sonographie (Hirngefäße) & fakultativ & $\checkmark$ & $\checkmark$ & $645^{\star}$ & 68,20 \\
\hline $\begin{array}{l}\text { - Duplex-Sonographie (Hirngefäße) } \\
\text { GOÄ Nrn. } 645+410+420 \text { (420 bis } \\
\text { zu 3x) }\end{array}$ & & & & $\begin{array}{l}645^{*} \\
+410 \\
+420\end{array}$ & $\begin{array}{l}68,20 \\
26,81 \\
10,73\end{array}$ \\
\hline \multicolumn{6}{|l|}{ 5. Röntgenuntersuchungen } \\
\hline Brustorgane (Thorax) & fakultativ & $\checkmark$ & 4 & $5137^{*}$ & 47,21 \\
\hline Mammographie & fakultativ & fakultativ & $\begin{array}{l}\text { ab } 40 \mathrm{~J} . \text { alle } \\
2 \mathrm{~J} . / \mathrm{ab} 50 \mathrm{~J} . \\
\text { jährlich }\end{array}$ & $\begin{array}{l}5265^{\star} \\
5266^{\star} \\
5267^{\star}\end{array}$ & $\begin{array}{l}31,48 \\
47,21 \\
15,74\end{array}$ \\
\hline Mammosonographie & & & & $\begin{array}{l}418 \\
420\end{array}$ & $\begin{array}{l}28,15 \\
10,72\end{array}$ \\
\hline $\begin{array}{l}\text { Osteodensitometrie mit quant. CT } \\
\text { oder digitaler Röntgentechnik }\end{array}$ & fakultativ & - & - & $5380^{*}$ & 31,48 \\
\hline $\begin{array}{l}\text { Osteodensitometrie mittels Dual- } \\
\text { Photonen-Absorptionstechnik }\end{array}$ & & & & $5475^{\star}$ & 31,48 \\
\hline \multicolumn{4}{|l|}{ 6. Laboruntersuchungen } & \multicolumn{2}{|c|}{1,15 fach (L) } \\
\hline Blutentnahme & $\checkmark$ & $\checkmark$ & $\checkmark$ & $250^{*}$ & $5,36(\mathrm{~L})$ \\
\hline BSG & $\checkmark$ & $\checkmark$ & - & $3501^{*}$ & $4,02(\mathrm{~L})$ \\
\hline CRP & & & & $3741^{*}$ & $13,41(\mathrm{~L})$ \\
\hline Differentialblutbild & $\checkmark$ & $\checkmark$ & $\checkmark$ & $3551^{*}$ & 1,345 (L) \\
\hline Glukose & $\checkmark$ & $\checkmark$ & $\checkmark$ & $3560^{*}$ & 2,68 (L) \\
\hline $\mathrm{HbA1c}$ & & & & $3561^{*}$ & 13,41 (L) \\
\hline Elektrolyte & $\checkmark$ & $\checkmark$ & $\checkmark$ & & \\
\hline - Natrium & & & & $3558^{*}$ & 2,01 (L) \\
\hline - Kalium & & & & $3557^{*}$ & 2,01 (L) \\
\hline Blutfette & $\checkmark$ & $\checkmark$ & $\checkmark$ & & \\
\hline - Chlolesterin & & & & 3562.H1* & 2,68 (L) \\
\hline - Triglyceride & & & & 3565.H1* & 2,68 (L) \\
\hline
\end{tabular}




\begin{tabular}{|c|c|c|c|c|c|}
\hline Ärztliche Leistungen & $\begin{array}{l}\text { General- } \\
\text { Check } \\
\text { (IGEL) }\end{array}$ & $\mathrm{DKD}^{\star *}$ & $\begin{array}{l}\text { Mayo- } \\
\text { Clinic*** }\end{array}$ & GOÄ Nrn. & $\begin{array}{l}{ }^{*} 1,8 / 2,3- \\
\text { fach } €\end{array}$ \\
\hline - HDL-Cholesterin & & & & 3563.H1* & 2,68 (L) \\
\hline - LDL-Cholesterin & & & & 3564.H1* & 2,68 (L) \\
\hline Leberwerte & $\checkmark$ & $\checkmark$ & $\checkmark$ & & \\
\hline - Alkalische Phosphatase & & & & 3587. H1* & $2,68(L)$ \\
\hline - Bilirubin gesamt & & & & 3581.H1* & 2,68 (L) \\
\hline - Serumelektrophorese & & & & $3574^{*}$ & 13,41 (L) \\
\hline - Gesamteiweiss & & & & 3573.H1* & $2,01(L)$ \\
\hline - Gamma-GT & & & & 3592.H1* & 2,68 (L) \\
\hline$-G L D H$ & & & & 3593.H1* & 3,35 (L) \\
\hline - GOT & & & & 3594.H1* & $2,68(L)$ \\
\hline$-G P T$ & & & & 3595.H1* & $2,68(L)$ \\
\hline Nierenwerte & $\checkmark$ & $\checkmark$ & $\checkmark$ & & \\
\hline - Kreatinin & & & & 3585.H1* & 2,68 (L) \\
\hline Harnsäure & $\checkmark$ & $\checkmark$ & $\checkmark$ & 3583.H1 & 2,68 (L) \\
\hline \multicolumn{6}{|l|}{ Schilddrüsenfunktion } \\
\hline$-T 3$ & $\checkmark$ & $?$ & $\checkmark$ & 4022.H4* & $16,76(\mathrm{~L})$ \\
\hline$-T 4$ & $\checkmark$ & $?$ & $\checkmark$ & 4023. H4* & $16,76(\mathrm{~L})$ \\
\hline$-T S H$ & $\checkmark$ & $?$ & $\checkmark$ & 4030.H4* & $16,76(\mathrm{~L})$ \\
\hline $\begin{array}{l}\text { Prostata-spezifisches Antigen } \\
\text { (Männer) }\end{array}$ & fakultativ & $\checkmark$ & - & $3908^{*}$ & $20,11(\mathrm{~L})$ \\
\hline $\begin{array}{l}\text { Teststreifen zur Feststellung: Blut im } \\
\text { Stuhl }\end{array}$ & $\checkmark$ & $\checkmark$ & $\checkmark$ & $3500^{*}$ & $6,03(L)$ \\
\hline Urinteststreifen & $\checkmark$ & $\checkmark$ & $\checkmark$ & $3511^{*}$ & $3,35(\mathrm{~L})$ \\
\hline Atemtest auf H. pylori & fakultativ & - & - & $4234^{*}$ & $6,03(L)$ \\
\hline \multicolumn{6}{|l|}{ 7. Abschließende Maßnahmen } \\
\hline $\begin{array}{l}\text { Ausführliche Erörterung der Untersu- } \\
\text { chungsergebnisse - }\end{array}$ & $\checkmark$ & $\checkmark$ & $\checkmark$ & 34 & 78,66 \\
\hline $\begin{array}{l}\text { Beratung-Angebote, z. B. mehr Bewe- } \\
\text { gung, Gewichtsreduktion, Alkoholre- } \\
\text { duktion, Nikotinabstinenz }\end{array}$ & & & & 3 & 20,11 \\
\hline - Abschlussbericht & $\checkmark$ & $\checkmark$ & $\checkmark$ & 80 & 40,22 \\
\hline Kosten in Euro ca. & $\begin{array}{c}180,- \text { bis } \\
360,-\end{array}$ & $\begin{array}{l}895,- \\
1500,-\end{array}$ & ca. $1.530,-$ & & \\
\hline
\end{tabular}

(Kursiv sind die zusätzlichen Vorschläge der Autoren gekennzeichet

* 1,8facher Satz $(\mathrm{L})=$ Labor 1,15 facher Satz

** Manager-Check- DKD (Deutsche Klinik für Diagnostik, Wiesbaden)

${ }^{\star * \star}$ Executive Health Programm (Mayo-Klinik, Rochester, Minnesota, USA)

Abrechnungstipp: Überall dort, wo erschwerte Untersuchungen oder besondere Untersuchungsverhältnisse vorliegen, ist eine Erhöhung vom Schwellenwert 2,3fach bis zum 3,5fachen Satz möglich und bei Leistungen mit reduziertem Gebührenrahmen (mit * gekennzeichnet) ist eine Erhöhung. vom Schwellenwert 1,8*fach bis zum 2,5fachen Satz möglich. Die entsprechenden Begründungen sind anzugeben. 


\section{General Check-up: Leistungsangebote von Kliniken}

Die Autoren haben beispielhaft die Leistungsangebote zur Prävention einiger Kliniken aus dem Internet zusammengetragen und nachfolgend fast immer in den Originalangaben -siehe eingerückte Texte - aufgeführt. Diese Übersicht dürfte niedergelassenen Ärzten helfen, ihr Leistungsangebot zum General- oder Manager Check up für ihre Patienten unter Einbezug von Kollegen verschiedener Fachrichtungen aus Praxis und Klinik zu definieren.

\section{- Deutsche Klinik für Diagnostik, Wiesbaden - www.dkd-wiesbaden.de}

Diese Klinik hat als eine der ersten in Deutschland den General-oder Manager Check eingeführt.

Basis-Programm für Männer und Frauen

- Eingangsgespräch/-untersuchung

- Basis-Laboruntersuchung (incl. Nüchtern-Plasma-Glucose)

- Globale Herz-Kreislauf-Risikoabschätzung

- Ruhe- und Belastungs-Elektrokardiographie (EKG)

- Ultraschall Herz

- Ultraschall hirnversorgende Arterien

- Ultraschall Bauchorgane

- Lungenfunktion (Ruhe-Spirometrie)

- Allergie-Test

- Augen-fachärztliche Basisuntersuchung

- Abschlussgespräch

- Ärztlicher Abschlussbericht

Bei Frauen wird zusätzlich eine frauenärztliche Untersuchung/Beratung durchgeführt.

\section{Zusätzliche altersabhängige Untersuchungen Männer}

ab 40 Jahre Stuhluntersuchung auf occultes Blut

ab 45 Jahre Laboruntersuchung PSA (= Prostata-spezifisches Antigen) und Ultraschall Prostata transrektal (wenn PSA pathologisch)

ab 50 Jahre Sigmoidoskopie (Darmspiegelung $60 \mathrm{~cm}$ )

ab 55 Jahre Totale Koloskopie (Spiegelung gesamter Dickdarm)

\section{Frauen}

ab 20 Jahre Zervixabstrich und transvaginale Sonographie

ab 40 Jahre Stuhluntersuchung auf occultes Blut

ab 50 Jahre Sigmoidoskopie (Darmspiegelung $60 \mathrm{~cm}$ ) und Mammographie und Mammasonographie

ab 55 Jahre Totale Koloskopie (Spiegelung gesamter Dickdarm)

Der Arzt schlägt ggf. nach dem Erstgespräch weitere notwendige Untersuchungen vor, wenn eine entsprechende Anamnese, ein Befund oder Vorbefund oder eine Begleiterkrankung vorliegen.

Der Check-up sollte in regelmäßigen Abständen wiederholt werden. Sollten bei Check-up-Erstuntersuchung auffällige Befunde erhoben worden sein, empfehlen wir, diese alle zwei Jahre durchführen zu lassen:

- Ultraschall Herz

- Ultraschall hirnversorgende Arterien

- Ultraschall Bauchorgane

- Lungenfunktion (Ruhe-Spirometrie)

- Allergie-Test

- Augen-fachärztliche Basisuntersuchung

Sollten Normalbefunde erhoben worden sein, hängt die Wiederholungsuntersuchung nach zwei Jahren von der Indikationsstellung des Arztes ab.

Bei Vorliegen eines Bluthochdrucks (> 140/90 mmHg oder blutdrucksenkende Therapie) sollten folgende Untersuchungen zusätzlich durchgeführt werden:

- Oraler Glucosetoleranztest

- 24-h-Blutdruckmessung (Indikationsstellung durch persönlichen Arzt)

- Verschlussdruck Beinarterien

- Ultraschall Beinarterien 
- Ultraschall Nierenarterien (Indikationsstellung durch persönlichen Arzt)

- Röntgen Brustorgane

- Untersuchung Augenhintergrund

- Ernährungsberatung

Bei Vorliegen einer Adipositas (Fettleibigkeit, Gewichtsindex $>30 \mathrm{~kg} / \mathrm{m}^{2}$ ) sollten folgende Untersuchungen zusätzlich durchgeführt werden:

- Oraler Glucosetoleranztest

- Körperfettbestimmung/-verteilung

- Ernährungsberatung

Bei Vorliegen von Fettstoffwechselstörungen (Cholesterin $>200 \mathrm{mg} / \mathrm{dl}$, Triglyceride $>150 \mathrm{mg} / \mathrm{dl}$, $\mathrm{LDL}>130 \mathrm{mg} / \mathrm{dl}, \mathrm{HDL}>40 \mathrm{mg} / \mathrm{dl}$ ) sollten folgende Untersuchungen zusätzlich durchgeführt werden:

- Oraler Glucosetoleranztest

- Ultraschall hirnversorgende Arterien

- Verschlussdruck Beinarterien

- Ultraschall Beinarterien

- Ernährungsberatung

Bei Vorliegen eines Diabetes sollten folgende Untersuchungen zusätzlich durchgeführt werden:

- 24-h-Blutdruckmessung

- Ultraschall Herz (Echokardiographie in Ruhe)

- Ultraschall Herz (Echokardiographie unter Belastung)

- Myokardszintigraphie

- Ultraschall hirnversorgende Arterien

- Verschlussdruck Beinarterien

- Ultraschall Beinarterien

- Röntgen Brustorgane (Indikationsstellung durch persönlichen Arzt)

- Untersuchung Augenhintergrund

- Diagnostik bei erektiler Dysfunktion (Impotenz)

- Ernährungsberatung

Gerne führen wir auf Ihren Wunsch (auch ohne medizinische Indikation) auch nachfolgende Untersuchungen durch:

- Spiroergometrie mit Laktatmessung/Fitnesstest

- Knochendichtemessung

- Körperfettbestimmung/-verteilung

- Hörtest

- HNO-fachärztliche Untersuchung

- Urologische Untersuchung/Beratung

- Hautärztliche Untersuchung/Beratung

- Orthopädische Untersuchung/Beratung

- Ernährungsberatung

- Reiseprophylaktische Beratung

Diagnoseklinik, München - www.diagnoseklinik-muenchen.de

Die Diagnoseklinik in München zeigt in ihrem „übervollen“ Internetauftritt im linken Menü zahlreiche Untersuchungen und Untersuchungsbereiche sowie u. a. auch einen Bereich „Therapie“. Eine stichwortartige Struktur für einen General-Check haben wir nicht gefunden - dafür aber einen BusinessCheck -, doch gibt es zu den einzelnen Begriffen viele - ein bisschen zu viele - weiterführende Informationen. Die Fülle der Information kann durchaus zu eigenen Angeboten animieren.

Diagnostik-Zentrum Fleetinsel, Hamburg - www.diagnostik-zentrum.de

Die Übersicht über den Basis-Check up ist unserer Meinung die im Layout beste und übersichtlichste Darstellung aller hier vorgestellten Internetauftritte; denn auf einen Blick ist hier zu erkennen, welche Untersuchungen in welchem Zeitrahmen durchgeführt werden: 


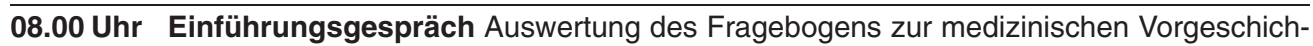 te und körperliche Untersuchung
08.45 Uhr Blutentnahme Großes Blutbild, Leber-, Gallen-, Nierenwerte, Blutfette, Blutzucker, Schilddrüsenwerte, Tumormarker etc.
09.00 Uhr Untersuchung der Bauchorgane und Schilddrüse Farbultraschall-Untersuchungen von Leber, Milz, Nieren, Bauchspeicheldrüse, Prostata (Männer), Uterus (Frauen) und der Schilddrüse

10.00 Uhr Gefäßdiagnostik Farbultraschall-Untersuchung der großen Arterien (Hirn-, Bein-Arterien, sowie Bauchhauptschlagader)

10.30 Uhr Herz-Kreis- und Lungen-Diagnostik Farbultraschall des Herzen, (3D Echokardiographie), EKG in Ruhe und unter Belastung, Prüfung der körperlichen Fitness (Spiro-ergometrie, Lungenfunktionsprüfung (Spirometrie), Körperfettgehalt (Body-Mass-Index)

$\overline{11.00}$ Uhr Hautvorsorge Vollständige Inspektion der Haut, Melanomscreening, Hauttypbestimmung

$\overline{\text { 12.00 Uhr Augen und Ohren Prüfung der Sehschärfe und des Gesichtsfeldes, Spiegelung des }}$ Augenhintergrundes, Messung des Augeninnendrucks, Gehörprüfung

13.00 Uhr Ergänzende Zusatzuntersuchungen wie Darmkrebsvorsorge (Coloskopie), Lungenscreening für Raucher (CT-Thorax)

14.30 Uhr Abschlussgespräch Besprechung der Befunde ggf. Vorschlag weiterführender Maßnahmen

Ergänzende Untersuchungen im Rahmen des Checks:

- Lungenscreening (low-dose CT), wenn Sie Raucher sind

- Darmspiegelung, wenn Sie über 45 Jahre alt sind und bisher noch keine Untersuchung auf Darmkrebs durchgeführt wurde.

- Bei höherem Herz-Kreislauf Risikoprofil Kalk-Scoring der Herzkranzgefäße ggf. CT-/MR-Diagnostik der Arterien.

- Gynäkologische Vorsorgeuntersuchung für Frauen inkl. Mammographie

- Magenspiegelung (Gastroskopie) bei Oberbauchbeschwerden durch unseren Facharzt für Gastroenterologie

- Funktionsprüfung des Bewegungsapparates bei Wirbelsäulen- und Gelenk-Beschwerden durch Fachärzte für Orthopädie

- Knochendichtemessung durch unseren Facharzt für Radiologie (CT-Diagnostik)

- Abklärung von rheumatologischen Fragestellung durch den Facharzt für Rheumatologie

- Individuelle Ernährungsberatung z. B. zur Gewichtsreduktion, mit einem auf Sie persönlich zugeschnittenen Trainingskonzept

- Zahnärztliche Untersuchung, Abklärung von Zusammenhängen mit orthopädischen oder HNOErkrankungen

- HNO-Untersuchung von Nase, Rachen und Kehlkopf, Allergietestung

- Neurologische Untersuchung zur Eruierung der Ursachen von z. B. Kopfschmerzen, Migräne und Schwindel

- Psychologische Gesprächsangebote zum Thema Schlafstörung, Stressbewältigung und Entspannungsmethoden

- Brain-Check zur Überprüfung von Gedächtnis und Konzentration

In einem ausführlichen Abschlussgespräch werden vom leitenden Arzt alle Ergebnisse zusammengefasst, erläutert und bewertet und ggf. weiterführende Untersuchungen besprochen. Ca. 14 Tage nach dem Check-Up erhalten die Patienten einen ausführlichen schriftlichen Bericht mit allen Ergebnissen und Daten.

Max-Grundig-Klinik, Baden-Baden - www.grundig-klinik.de

Die Max Grundig Klinik bietet unterschiedlich intensive Vorsorge-Untersuchungen an. Der Basis Check-up dauert ca. $1^{1 / 2}$ bis 2 Tage, der Erweiterte Check-up ca. $2^{1 / 2}$ bis 3 Tage. Folgende Untersuchungen werden durchgeführt: 
Basis Check-up

- Persönliche Hinterfragung der Vorerkrankungen einschließlich der familiären Erkrankungsrisiken

- Körperliche Untersuchung

- Ruhe-EKG

- Ergometrie (Belastungs-EKG)

- Röntgen Thorax p.a. (Röntgenaufnahme der Lunge)

- Farbdoppler-Echokardiographie (Ultraschall des Herzens)

- Abdomensonographie (Ultraschall der Bauchorgane)

- Schilddrüsensonographie (Ultraschall)

- Spirometrie (Lungenfunktionsprüfung)

- Digitale Untersuchung von Mastdarm und Prostata

- Sigmoidoskopie (kleine Darmspiegelung)

Zusätzlich gehören zum Basis Check-up in der Max Grundig Klinik auch folgende Laboruntersuchungen:

- Natrium, Kalium, Calcium,

- Harnsäure,

- Kreatinin,

- Gesamt-Cholesterin, Triglyzeride, HDL-Cholesterin, LDL-Cholesterin,

- Blutzucker,

- GPT,

- Gamma-GT,

- Alkalische Phosphatase,

- Amylase,

- PTT und Quick,

- Gesamteiweiß, Eiweißelektrophorese,

- Blutbild, Differentialblutbild,

- BSG,

- PSA, CA19-9, CRP, CA15-3,

- Urinstatus,

- Stuhl auf Blutbeimengung.

Im Rahmen dieses Programms werden mit Hilfe der angeführten technischen und laborchemischen Untersuchungen die Funktionen der Organsysteme

- Herz

- Lunge

- Schilddrüse

- Leber

- Magen, Darm und Bauchspeicheldrüse

überprüft und die wichtigsten Risikofaktoren (Blutfette, Blutzucker, Blutdruck) sowie einige Tumormarker definiert. Ein ausführliches Gespräch mit dem Arzt sowie ein detaillierter schriftlicher Bericht über die diversen Befunde beschließen den Basis Check-up.

Nach Wunsch oder besonderer Befundlage können Sie in das Basisprogramm Ergänzungsuntersuchungen (z. B. Virusscreening, Immun- und Hormonstatus, MR-Tomographien etc.) mit aufnehmen lassen.

\section{Erweiterter Check-up}

Folgende Untersuchungen werden durchgeführt:

- Persönliche Hinterfragung der Vorerkrankungen einschließlich der familiären Erkrankungsrisiken

- Eingehende körperliche und orientierend neurologische Untersuchung

- Ruhe-EKG

- Ergometrie (Belastungs-EKG)

- Röntgen Thorax in zwei Ebenen (Röntgenaufnahme der Lunge)

- Schilddrüsensonographie (Ultraschall)

- Spirometrie (Lungenfunktionsprüfung)

- Digitale Untersuchung von Mastdarm und Prostata

- Vollständige Koloskopie

- Gastroskopie 
E. Individuelle Gesundheitsleistungen von A - Z

- Sonographie der Bauchorgane einschl. des Urogenitaltraktes

- Echokardiographie

- Osteodensitometrie (Knochendichte)

- Langzeit-EKG

- Langzeit Blutdruckmonitoring

- Mammographie

- Sonographie der Mammae

- Quantitative Körperfettmessung

Zusätzlich gehören zum erweiterten Check-up in der Max Grundig Klinik auch folgende Laboruntersuchungen: Natrium, Kalium, Calcium, Bilirubin, Harnsäure, Kreatinin, Harnstoff, Gesamt-Cholesterin, Triglyzeride, HDL-Cholesterin, LDL-Cholesterin, Blutzucker, Quick, PTT, GOT, GPT, Gamma-GT, Cholinesterase, AP, CK, LDH, Amylase, Lipase, CRP, Gesamteiweiß, Eiweißelektrophorese, Blutbild, Differentialblutbild, BSG, fT3, fT4, TSH basal, PSA, CA15-3, CA19-9, Urinstatus, Urinsediment. Im Rahmen dieses Programms werden mit Hilfe der angeführten technischen und laborchemischen Untersuchungen die Funktionen der Organsysteme Herz, Lunge, Schilddrüse, Leber, Magen, Darm und Bauchspeicheldrüse überprüft und die wichtigsten Risikofaktoren (Blutfette, Blutzucker, Blutdruck) sowie einige Tumormarker definiert. Ein ausführliches Gespräch mit dem Arzt sowie ein detaillierter schriftlicher Bericht über die diversen Befunde beschließen den Erweiterten Check-up.

Nach Wunsch oder besonderer Befundlage können sich Patienten in das Erweiterte Programm noch Ergänzungsuntersuchungen wie z. B. Virusscreening, Immun- und Hormonstatus, MR-Tomographien mit aufnehmen lassen.

\section{Krebs-Früherkennungsuntersuchungen und -Verlaufskontrollen}

Bei den GKV-Kassen bestehen für die Früherkennungsuntersuchungen von Krebserkrankungen und alle Vorsorgeuntersuchungen zahlreiche Einschränkungen z. B.

- Alter der/des Versicherten

- festgelegte Zielkrankheiten

- Häufigkeit der Untersuchungen

- festgelegte Zeiträume der Untersuchungen

- Umfang der Untersuchung

Es wird heutzutage sehr viel von dem mündigen Patienten gesprochen, der Entscheidungen zu seiner Gesundheit selbst treffen kann und soll. Mit den IGEL-Leistungen ist nun die Möglichkeit gegeben, dass sich Patienten zusammen mit dem Arzt für zusätzliche, individuelle und ausgedehnte Früherkennungsuntersuchungen und/oder Vorsorge-Untersuchungen entscheiden.

Bei den GKV-Kassen können Untersuchungen zur Früherkennung von Krebserkrankungen bei Frauen ab dem 20. und bei Männern ab dem 45. Lebensjahr durchgeführt werden.

Im Rahmen der GOÄ gibt es keine Krebsfrüherkennungs-Richtlinien wie sie der Bundesausschuss der Ärzte und Krankenkassen (G-BA) für GKV-Patienten aufgestellt hat. Immer mehr private Krankenkassen und auch die Beihilfen orientieren sich deshalb an den Bestimmungen und vorgeschriebenen Leistungen der gesetzlichen Krankenversicherungen. Siehe 9.1. und 9.2.

Nach den Richtlinien des Bundesausschusses der Ärzte und Krankenkassen (GKV) gelten nach EBM für beide Geschlechter zur Darmkrebsfrüherkennung folgende Untersuchungen:

\begin{tabular}{|l|l|l|}
\hline Alter & Art der Untersuchung & Turnus \\
\hline $50-54$ & Test auf okkultes Blut & jährlich \\
\hline ab 55 & $\begin{array}{l}\text { Test auf okkultes Blut nur, wenn das Koloskopieangebot nicht in } \\
\text { Anspruch genommen wird }\end{array}$ & 2-jährlich \\
\hline ab 55 & Koloskopie & $\begin{array}{l}\text { 2. Koloskopie 10 Jah- } \\
\text { re nach der ersten }\end{array}$ \\
\hline
\end{tabular}

Krebsvorsorgeuntersuchung bei der Frau

Nach den Richtlinien des Bundesausschusses der Ärzte und Krankenkassen gelten für die präventiven Untersuchungen der Frauen folgende Daten:

- ab dem 20. Lebensjahr = Früherkennungsuntersuchung Genitalkrebs

- $a b$ dem 30. Lebensjahr = Früherkennungsuntersuchung Genitalkrebs und Brustkrebs und Krebserkrankung der Haut 
- ab dem 45. Lebensjahr = Früherkennungsuntersuchung Genital-, Brust-, Haut-, Rektum- und Dickdarmkrebs

- $a b$ dem 56. Lebensjahr = Anspruch auf eine 1. Koloskopie und eine 2. frühestens 10 Jahre danach.

\begin{tabular}{|l|l|r|r|}
\hline GOÄ Nr. & Kurzlegende & $\mathbf{1}$ fach $€$ & $\mathbf{2 , 3 f a c h} €$ \\
\hline $\mathbf{2 7}$ & Krebsvorsorge bei einer Frau & 18,65 & $\mathbf{4 2 , 9 0}$ \\
\hline
\end{tabular}

Weitere zusätzliche Untersuchungen finden Sie im Kapitel Gynäkologie auf Seite XXX.

Krebsvorsorgeuntersuchung beim Mann

Männer haben gemäß den Richtlinien des Bundesausschusses der Ärzte und Krankenkassen nach EBM folgenden Anspruch:

- ab Beginn des 45. Lebensjahres die jährliche Untersuchung zur Früherkennung von Krebserkrankungen der Prostata, des äußeren Genitales und der Haut,

- ab dem 50. Lebensjahr zusätzlich die des Rektums und des übrigen Dickdarms.

- ab dem 56. Lebensjahr eine 1. Koloskopie und eine 2. frühestens 10 Jahre danach.

\section{Zusätzliche Krebsvorsorge beim Mann}

Erfolgt eine digitale rektale Untersuchung der Prostata zum Ausschluss eines Karzinoms auf Wunsch des Patienten ausserhalb einer Krebsvorsorgeuntersuchung so könnten alternativ - statt der GOÄ Nr. 28 und abhängig von der erbrachten Leistung - berechnet werden:

\begin{tabular}{|l|l|r|r|}
\hline GOÄ Nr. & Kurzlegende & $\mathbf{1 f a c h} €$ & $\mathbf{2 , 3 f a c h} €$ \\
\hline $\mathbf{2 8}$ & Krebsvorsorge bei einem Mann ${ }^{1}$ & 16,32 & $\mathbf{3 7 , 5 4}$ \\
\hline $\mathbf{5}$ & Symptombezogene Untersuchung & 4,66 & $\mathbf{1 0 , 7 3}$ \\
\hline $\mathbf{6}$ & $\begin{array}{l}\text { Unterschung Nieren, harnableitende Wege, zusätzl. Untersu- } \\
\text { chung Prostata, Prüfung Bruchpforten, Hoden und Nebenhoden }\end{array}$ & 5,83 & $\mathbf{1 3 , 4 1}$ \\
\hline $\mathbf{7}$ & Bauchorgane & 9,33 & $\mathbf{2 1 , 4 5}$ \\
\hline $\mathbf{8}$ & Ganzkörperstatus & 15,15 & $\mathbf{3 4 , 8 6}$ \\
\hline $\mathbf{1 1}$ & Mastdarm u./o. der Prostata - Digitaluntersuchung & 3,50 & $\mathbf{8 , 0 4}$ \\
\hline $\mathbf{3 5 0 0 ^ { * }}$ & Stuhl auf Blut, 3mal & 5,25 & $\mathbf{6 , 0 3}$ \\
\hline
\end{tabular}

* nur 1,15 facher Satz

1) $\mathrm{Nr}$. 28 nicht neben $5-8,11$ und 3500 abrechenbar

Früherkennung Prostata-Karzinom

\begin{tabular}{|c|c|c|c|}
\hline GOÄ Nr. & Kurzlegende & 1 fach $€$ & $2,3 f a c h €$ \\
\hline 410 & Ultraschalluntersuchung Prostata & 11,66 & 26,81 \\
\hline $420(2 x)$ & Ultraschalluntersuchung der Nieren rechts und links & $\begin{array}{r}4,66 \\
(2 x) \\
\end{array}$ & $10,72(2 x)$ \\
\hline 420 & Ultraschalluntersuchung ein weiteres Organ & 4,66 & 10,72 \\
\hline 403 & Transrektale Ultraschalluntersuchung & 8,74 & 15,74 \\
\hline \multirow[t]{2}{*}{$250^{*}$} & Blutentnahme & 2,33 & 4,20 \\
\hline & Labor & 1 fach $€$ & ${ }^{\star} 1,15$ fach $€$ \\
\hline $3550^{* *}$ & Blutbild & 3,50 & 4,02 \\
\hline $3551^{* \star}$ & Differential Blutbild & 1,17 & 1,34 \\
\hline $3511^{\star *}$ & Urin-Teststreifen & 2,91 & 3,35 \\
\hline $3908^{\star \star}$ & PSA Blutuntersuchung & 17,49 & 20,11 \\
\hline
\end{tabular}


E. Individuelle Gesundheitsleistungen von A - Z

Krebsfrüherkennungsuchungen für spezielle Krebsarten

Früherkennung bösartiger Erkrankungen im Nasen-Rachen-Raum bei Risikopersonen

\begin{tabular}{|l|l|r|r|}
\hline GOÄ Nr. & Kurzlegende & $\mathbf{1 f a c h} €$ & $\mathbf{2 , 3 f a c h} €$ \\
\hline $\mathbf{1 5 3 0}$ & Laryngoskopie & 10,61 & $\mathbf{2 4 , 4 0}$ \\
\hline $\mathbf{1 5 3 3}$ & Schwebe- oder Stützlaryngoskopie & 29,14 & $\mathbf{6 7 , 0 3}$ \\
\hline $\mathbf{1 5 3 4}$ & PE aus dem Kehlkopf & 26,99 & $\mathbf{6 2 , 0 7}$ \\
\hline $\mathbf{1 4 1 8}$ & $\begin{array}{l}\text { Endoskopie der Nasenhaupthöhlen und/oder des Nasenra- } \\
\text { chenraumes }\end{array}$ & 10,49 & $\mathbf{2 4 , 1 3}$ \\
\hline
\end{tabular}

Früherkennung des Bronchialkarzinoms

\begin{tabular}{|c|c|c|c|}
\hline GOÄ Nr. & Kurzlegende & 1 fach $€$ & $\begin{array}{r}{ }^{*} 1,8 / \\
2,3 \mathrm{fach} €\end{array}$ \\
\hline 3 & $\begin{array}{l}\text { Eingehende Beratung (mind. } 10 \text { Min.) -nicht neben Sonderleis- } \\
\text { tungen }\end{array}$ & 8,74 & 20,11 \\
\hline $5135^{\star}$ & Röntgen - Thorax eine Ebene & 16,35 & 29,38 \\
\hline $5137^{*}$ & $\begin{array}{l}\text { Röntgen - Thorax ggf. mit Breischluck und Durchleuchtung in } \\
\text { mehreren Ebenen }\end{array}$ & 26,23 & 47,21 \\
\hline 5139* & Teil der Brustorgane & 10,49 & 18,99 \\
\hline 677 & Bronchoskopie oder Thorakospie & 34,97 & 80,44 \\
\hline 678 & Bronchoskopie mit zusätzl. operativen Eingriff z. B. PE & 52,46 & 120,65 \\
\hline
\end{tabular}

* Zytopathologische Untersuchung des Sputums bei langjährigen Rauchern ab 45 Jahren

Geeignet für ein Massen-screeeing ist die automatisierten Sputum-Zytometrie (ASZ). Hier bei der DNA-Gehalt der Zellkerne untersucht und in ein Verhältnis zur gesamten Zellstruktur gesetzt. Dabei finden sich Muster, die als pathologisch oder nicht pathologisch zu werten sind.

Die Zelluntersuchung geschieht computergestützt und vollautomatisch, deshalb können große Sputum-Mengen analysiert werden. Fachleute halten das ASZ für mindestens so spezifisch und sensitiv wie die konventionelle Sputum-Zytologie.

Weitere Diagnostik:

- Fluoreszenz-Bronchoskopie

- Endobronchialer Ultraschall (EBUS)

Informationen zur Vorsorge des Bronchialkarzinom mit zytopathologischen Untersuchungen des Sputums erhalten Sie und Ihre Patienten im Internet unter den Adressen:

- www.med.uni-duesseldorf.de/CytoPathologie/index.html

- www.lungenkrebsvorsorge.de

Früherkennung des Colonkarzinoms

Frauen und Männern bieten die gesetzlichen und privaten Krankenversicherungen zu Darmkrebs-

Früherkennung an

- vom 50. bis 55. Lebensjahr einmal jährlich ein Test auf verborgenes Blut im Stuhl

- ab dem 56. Lebensjahr Angebot einer ersten Darmspiegelung (Koloskopie) und Wiederholung dieser Vorsorgeuntersuchung zehn Jahre nach der ersten Untersuchung; der Stuhlblut-Test wird damit in diesem Alter überflüssig

- für diejenigen Versicherten, die die Darmspiegelung nicht in Anspruch nehmen wollen oder können: Angebot eines zweijährlichen Stuhlblut-Tests ab dem 56. Lebensjahr

- der Stuhlblut-Test kann auch unabhängig von einer anderen Krebs-Früherkennungs-untersuchung wahrgenommen werden

Innerhalb des langen Zeitraumes von zehn Jahren bis zur Wiederholung der Coloskopie, wünschen viele Patienten eine Kontrolle in kürzerem Abstand als IGEL-Leistung. 


\begin{tabular}{|l|l|r|r|}
\hline GOÄ Nr. & Kurzlegende & $\mathbf{1 f a c h} €$ & $\mathbf{2 , 3 f a c h ~} €$ \\
\hline $\mathbf{3}$ & $\begin{array}{l}\text { Eingehende Beratung (mind. 10 Min.) - nicht neben Sonderleis- } \\
\text { tungen }\end{array}$ & 8,74 & $\mathbf{2 0 , 1 1}$ \\
\hline $\mathbf{6 9 0}$ & Rektoskopie - starr oder flexibel & 20,40 & $\mathbf{4 6 , 9 2}$ \\
\hline $\mathbf{6 8 9}$ & Sigmoidoskopie & 40,80 & $\mathbf{9 3 , 8 3}$ \\
\hline $\mathbf{6 8 7}$ & Hohe Koloskopie bis Coecum & 87,43 & $\mathbf{2 0 1 , 0 9}$ \\
\hline $\mathbf{6 8 8}$ & Partielle Koloskopie ggf. einschl. Rektoskopie & 53,46 & $\mathbf{1 2 0 , 6 5}$ \\
\hline
\end{tabular}

Wegen der geringen Sensitivität und Spezifität der Testbriefchen auf okkultes Blut ist eine immunologische Testung (Spezifität etwa $95 \%$ ) sinnvoll:

\begin{tabular}{|l|l|r|r|}
\hline GOÄ Nr. & Kurzlegende & $\mathbf{1 f a c h} €$ & $\begin{array}{r}\text { **1,15/ } \\
\mathbf{2 , 3 f a c h ~} €\end{array}$ \\
\hline $\mathbf{3}$ & $\begin{array}{l}\text { Eingehende Beratung (mind. 10 Min.) - nicht neben Sonderleis- } \\
\text { tungen }\end{array}$ & 8,74 & $\mathbf{2 0 , 1 1}$ \\
\hline $\mathbf{A 3 7 4 7} 7^{\star *}$ & $\begin{array}{l}\text { Hämoglobin, immunologisch - analog } \\
\text { GOÄ Kurztext Nr. 3747: Haptoglobin }\end{array}$ & 10,49 & $\mathbf{1 2 , 0 7}$ \\
\hline $\mathbf{A 3 7 4 7 * *}$ & $\begin{array}{l}\text { Hämoglobin - Haptoglobin - Komplex, immunologisch - analog } \\
\text { GOÄ Kurztext Nr. 3747: Haptoglobin }\end{array}$ & 10,49 & $\mathbf{1 2 , 0 7}$ \\
\hline
\end{tabular}

Früherkennung des Harnblasenkarzinoms

\begin{tabular}{|l|l|r|r|}
\hline GOÄ Nr. & Kurzlegende & $\mathbf{1 f a c h} €$ & $\mathbf{2 , 3 f a c h} €$ \\
\hline $\mathbf{3}$ & $\begin{array}{l}\text { Eingehende Beratung (mind. 10 Min.) - nicht neben Sonderleis- } \\
\text { tungen }\end{array}$ & 8,74 & $\mathbf{2 0 , 1 1}$ \\
\hline $\mathbf{1 7 8 5}$ & Zystoskopie & 12,07 & $\mathbf{2 7 , 7 5}$ \\
\hline $\mathbf{1 7 8 6}$ & Zystoskopie einschl. Gewebeentnahme & 20,69 & $\mathbf{4 7 , 5 9}$ \\
\hline
\end{tabular}

Weitere Diagnostik:

- Fluoreszenz-Zystoskopie

- Tumormarker NMP22 (Nuclear matrix protein 22)

Früherkennung des Hautkrebses

\begin{tabular}{|l|l|r|r|}
\hline GOÄ Nr. & Kurzlegende & $\mathbf{1 f a c h ~} €$ & $\mathbf{2 , 3 f a c h} \boldsymbol{\text { f }}$ \\
\hline $\mathbf{3}$ & $\begin{array}{l}\text { Eingehende Beratung (mind. 10 Min.) - nicht neben Sonderleis- } \\
\text { tungen }\end{array}$ & 8,74 & $\mathbf{2 0 , 1 1}$ \\
\hline $\mathbf{7}$ & Untersuchung der Haut & 9,33 & $\mathbf{2 1 , 4 5}$ \\
\hline $\mathbf{7 5 0}$ & Auflichtmikroskopie (Dermatoskopie) & 6,99 & $\mathbf{1 6 , 0 9}$ \\
\hline $\mathbf{6 1 2} \mathbf{A}$ & $\begin{array}{l}\text { Video-system-gestützte Untersuchung und Bilddokumentation } \\
\text { von Muttermalen - analog (Empfehlung der BÄK) - GOÄ § 6 (2) } \\
\text { GOÄ Kurztext Nr. 612: Ganzkörperplethysmographische Bestimmung der absolu- } \\
\text { ten und relativen Sekundenkapazität und des Atemwegwiderstandes... }\end{array}$ & 44,12 & $\mathbf{1 1 0 , 3 1}$ \\
\hline
\end{tabular}

Es erscheint sehr sinnvoll, dass der Dermatologe seinen Patienten über die für die Selbstuntersuchung geltende ABCD-Regel unterrichtet und inn damit sensibilisiert einen Leberfleck einzuschätzen und den Arzt aufzusuchen, wenn mindestens eines der folgenden Merkmale zutrifft:

A wie Asymmetrie

B wie Begrenzung

C wie colour

D wie Durchmesser

Zusätzlich Faktoren, auf die aufmerksam gemachen werden sollten, sind:

- Größenzunahme und

- Formveränderung vorhandener Leberflecken,

- Juckreiz und

- Blutung aus einem Mal. 
E. Individuelle Gesundheitsleistungen von A - Z

Auf einen Blick: Tumormarker zur Verlaufskontrolle und Diagnostik

Das Institut für Medizinische Diagnostik - eine Gemeinschaftspraxis für Labormedizin, Mikrobiologie, Transfusionsmedizin - in Berlin informiert unter www.imd-berlin.de/index.php?id=426 übersichtlich mit einer Liste der Tumormarker 1. und 2. Wahl - die wir freundlicherweise übernehmen durften - über Möglichkeiten zur Verlaufskontrolle und ggf. zur Tumor-Diagnostik.

Die folgende Leistungen werden in der Regel - nach Rücksprache mit dem Patienten -vom behandelnden Arzt beim Labor in Auftrag gegeben und dann vom Labor direkt beim Patienten liquidiert.

Die Liste wurde für das Buch alphabetisch nach den Organen geordnet.

\begin{tabular}{|c|c|c|}
\hline Organ & 1. Wahl & 2. Wahl \\
\hline $\begin{array}{l}\text { Bronchial-Ca: } \\
\text { - kleinzellig } \\
\text { - nicht kleinzellig } \\
\text { - Adenokarzinom } \\
\end{array}$ & $\begin{array}{l}\text { NSE } \\
\text { CYFRA 21-1 } \\
\text { CYFRA 21-1, CEA }\end{array}$ & $\begin{array}{l}\text { TPA, CA 125, CEA, HCT TPA, } \\
\text { CA 125, CEA, SCC TPA }\end{array}$ \\
\hline $\begin{array}{l}\text { Colon/Rektum } \\
\text { Dünndarm (Carzinoid) }\end{array}$ & $\begin{array}{l}\text { Hämoglobin/Haptoglobin im Stuhl CEA, } \\
\text { CA 19-9 } \\
\text { Serotonin, 5-HIES }\end{array}$ & $\begin{array}{l}\text { TPA } \\
\text { NSE }\end{array}$ \\
\hline $\begin{array}{l}\text { Gastrinom (Zollinger-Ellison-Syn- } \\
\text { drom) }\end{array}$ & Gastrin & - \\
\hline Harnblase & NMP-22, CYFRA 21-1, TPA & CA 19-9, CEA \\
\hline HNO/Kopf & CYFRA 21-1, SCC & CEA, EBV-EA, Ferritin \\
\hline Hoden & AFP, HCG, Plazenta-AP & SCC, M2-PK \\
\hline Hypernephrom & Erythropoietin & - \\
\hline Hypophyse & ACTH, Prolaktin, STH, TSH, FSH, LH & - \\
\hline $\begin{array}{l}\text { Leber/Galle: } \\
\text { - Leberzellkarzinom } \\
\text { - Lebermetastasen } \\
\text { - Gallengangskarzinom }\end{array}$ & $\begin{array}{l}\text { AFP, AFP-L3\%, DCP } \\
\text { CEA AFP } \\
\text { CA 19-9 }\end{array}$ & $\begin{array}{l}\text { DCP } \\
\text { TPA, CA 19-9, CA } 125 \text { CEA, } \\
\text { AFP, CA } 125\end{array}$ \\
\hline Magen & CEA, CA 72-4 & CA 19-9, CEA,TPA \\
\hline Mamma & CA 15-3, CEA & MCA, TPA \\
\hline Nebenschilddrüse & Parathormon (PTH) & - \\
\hline Niere & CEA, M2 PK & Renin, TPA \\
\hline NNR & Cortisol & Androgene, Östrogene \\
\hline Ösophagus & CEA, SCC & CA 19-9 \\
\hline Ovar & CA 125, CEA & $\begin{array}{l}\text { HCG, AFP, CA 72-4, CA 15-3, } \\
\text { TPA }\end{array}$ \\
\hline $\begin{array}{l}\text { Pankreas } \\
\text { - exkretorisch } \\
\text { - inkretorisch (Insulinom) }\end{array}$ & $\begin{array}{l}\text { CA 19-9 } \\
\text { Insulin, C-Peptid }\end{array}$ & $\begin{array}{l}\text { CA 50, CA 125, CA 72-4, } \\
\text { TPA, CEA }\end{array}$ \\
\hline Phäochromozytom & Katecholamine, VMS & - \\
\hline Prostata & PSA (ges. u. frei) & CEA, PAP, TPA \\
\hline $\begin{array}{l}\text { Schilddrüsenkarzinom: } \\
\text { - medullär } \\
\text { - Follikulär, papillär }\end{array}$ & $\begin{array}{l}\text { Calcitonin (HCT) } \\
\text { Thyreoglobulin }\end{array}$ & $\begin{array}{l}\text { NSE, CEA } \\
\text { TPA }\end{array}$ \\
\hline Uterus & CEA, SCC & CA 19-9, Ca 125 \\
\hline Lymphatisch/myeol. System & $\begin{array}{l}\text { 2-Mikroglobulin, } \\
\text { monokl. Immunglobuline }\end{array}$ & $\begin{array}{l}\text { Ferritin, Neopterin,Lysozym, } \\
\text { TK (Thymidinkinase) }\end{array}$ \\
\hline Sarkom & AP (Isoenzyme), Hydroxyprolin, Ostase & CEA, TPA \\
\hline Haut (Melanom) & S-100 & \\
\hline Ohne Organzuordnung & $\begin{array}{l}\text { p53-Auto-AK werden ausschl. bei Tumo- } \\
\text { ren gebildet - (Spezifität für eine Tm Er- } \\
\text { krankung: 100\%; (Sensitivität 10-40\%) }\end{array}$ & \\
\hline
\end{tabular}


Ohne Organzuordnung:

p53-Auto-AK werden ausschließlich bei Tumoren gebildet - (Spezifität für eine Tm-Erkrankung: 100\%; Sensitivität $10-40 \%$ )

GOÄ Nrn. der oben aufgeführten Tumormarker

\begin{tabular}{|c|c|}
\hline Tumormarker & GOÄ Nrn. \\
\hline B2-Mikroglobulin & $3754^{*}$ \\
\hline 5-HIES (5-Hydroxy-Indolessigsäure - 24h-SU) & $4071^{*}, 4080^{*}$ \\
\hline ACTH (Adrenocorticotropes Hormon im Plasma) & 4049* \\
\hline AFP (Alpha-Fetoprotein) & $3743^{*}$ \\
\hline AFP-L3\% (Alpha-Fetoprotein (AFP) L3\% and Total, Hepatocellular Carcinoma) & 4078 \\
\hline $\begin{array}{l}\text { Androgene } \\
\text { - Dehydroepiandrosteron (DHEA) } \\
\text { - Dihydrotestosteron } \\
\text { - Testosteron }\end{array}$ & $\begin{array}{c}4037^{*} \\
4069^{* 1} \\
4042^{*}\end{array}$ \\
\hline $\begin{array}{l}\text { AP (Isoenzyme - Alkalische Phosphatase Isoenzymbestimmung, Knochen-AP, intestinale AP, } \\
\text { Leber-AP) }\end{array}$ & $3784^{*}$ \\
\hline CA 125 (Cancer Antigen 125) & $3900^{*}$ \\
\hline CA 15-3 (Cancer-Antigen 15-3) & $3901^{*}$ \\
\hline CA 19-9 (Cancer-Antigen 19-9) & $3902^{*}$ \\
\hline CA 50 (Cancer-Antigen 50) & $3903^{*}$ \\
\hline CA 72-4 (Cancer-Antigen 72-4) & $3904^{*}$ \\
\hline Calcitonin (HCT humanes Kalzitonin) & $4047^{*}$ \\
\hline CEA (Carcinoembryonales Antigen) & $3905^{*}$ \\
\hline Cortisol & 4020* \\
\hline C-Peptid (CPEP, connecting peptide) & $4046^{*}$ \\
\hline CYFRA 21-1 (Cytokeratin 19-Fragmente) & $3906^{*}$ \\
\hline DCP (Des-Gamma-Carboxyprothrombin) - Tumormarker beim hepatozellulären Karzinom) & 4078 \\
\hline EBV-EA - Epstein-Barr-Virus-Antikörper - early antigen & $4311 * \mathrm{ff}$. \\
\hline Erythropoetin & 4050* \\
\hline Ferritin & $3742^{*}$ \\
\hline FSH (Follikelstimulierendes Hormon, (hypophysäres Gonadotropin) & $4021^{*}$ \\
\hline Gastrin & $4051^{*}$ \\
\hline $\begin{array}{l}\text { Hämoglobin/Haptoglobin im Stuhl } \\
\text { Immunchromatographischer Schnelltest zum qualitativen Nachweis von okkultem Blut im } \\
\text { Stuhl- (PreventID CC oder (IDEAL) } \\
\text { Hämoglobin-Haptoglobin-Komplex ELISA. Dieses Enzyme-Linked-Immuno-Sorbent-Assay } \\
\text { (ELISA) dient zur quantitativen Erfassung des Hämoglobin-Haptoglobin-Komplexes im Stuhl. }\end{array}$ & $\begin{array}{c}\text { A } 3571^{*} \\
\text { oder A } 3736^{*} \\
\text { analog } 3572^{*} \\
\text { oder analog } \\
3747 \\
\end{array}$ \\
\hline HCG (Human-Chorion-Gonadotropin) & $4024^{*}$ \\
\hline B-HCH (ß-Human-Chorion-Gonadotropin) & $4053^{*}$ \\
\hline Hydroxyprolin & 4078* \\
\hline Immunfixation (monokl. Immunglobuline, je Antiserum) & 3749 \\
\hline Insulin & $4025^{*}$ \\
\hline $\begin{array}{l}\text { Katecholamine } \\
\text { - Adrenalin (Plasma) } \\
\text { - Metanephrin (Plasma) } \\
\text { - Noradrenalin (Plasma) } \\
\text { - Normetanephrine (Plasma) }\end{array}$ & $\begin{array}{l}4072^{*} \\
4072^{*} \\
4072^{*} \\
4072^{*}\end{array}$ \\
\hline LH (Luteinisierendes Hormon, (hypophysäres Gonadotropin) & 4026* \\
\hline
\end{tabular}

1) Untersuchungen mit ähnlichem Aufwand 


\begin{tabular}{|c|c|}
\hline Tumormarker & GOÄ Nrn. \\
\hline Lysozym & $3793^{*}$ \\
\hline M2 PK (Isoenzym M2 der Pyruvatkinase oder Tumor M2-PK) & A 3903 \\
\hline MCA (mucin-like carcinoma-associated antigen) & A 3901 \\
\hline \multicolumn{2}{|l|}{ monokl. Immunglobuline } \\
\hline Neopterin & 4069* \\
\hline NMP-22 i. Urin (Nukleäres-Matrix-Protein 22) & $3911^{*}$ \\
\hline NSE (Neuronspezifische Enolase) & $3907^{*}$ \\
\hline Ostase & 4062 \\
\hline $\begin{array}{l}\text { Östrogene } \\
\text { - Össtradiol } \\
\text { - Össtron } \\
\text { - Östriol }\end{array}$ & $\begin{array}{l}4039^{*} \\
4044^{*} \\
4027^{\star}\end{array}$ \\
\hline p53-Auto-AK & 3877 \\
\hline $\begin{array}{l}\text { PAP (Saure Prostataphosphatase SPP, engl. Prostatic Acid Phosphatase PAP - beide } \\
\text { Abkürzungen in Deutschland üblich) } i\end{array}$ & $3794^{*}$ \\
\hline Parathormon (PTH) & $4056^{*}$ \\
\hline $\begin{array}{l}\text { Plazenta-AP (PLAP - plazentare alkalische Phosphatase, humane alkalische Plazenta- } \\
\text { Phosphatase) }\end{array}$ & 4069* \\
\hline Prolaktin & $4041^{*}$ \\
\hline PSA (ges. u. frei) (Prostataspezifisches Antigen (total) & 3908. H3* $^{*}$ \\
\hline Renin & $4058^{*}, 4115^{*}$ \\
\hline S-100 (Protein-S100B) & 4069* \\
\hline SCC (Squamous Cell Carcinoma Antigen) & $3909 *$ \\
\hline Serotonin & $4075^{*}$ \\
\hline STH (Wachstumshormon, somatotropes Hormon) & $4043^{*}$ \\
\hline Thyreoglobulin & 4070* \\
\hline TK (Thymidinkinase) & $3910^{*}$ \\
\hline TPA (Tissue Polypeptide Antigen) & $3911^{*}$ \\
\hline TSH (TSH-basal, Thyreotropin, Thyreoidea stimulierendes Hormon) & $4030^{*}$ \\
\hline VMS (Vanillinmandelsäure) & $4077^{*}, 4085^{\star}$ \\
\hline
\end{tabular}

\section{Praeoperative Diagnostik vor operativen Eingriffen außerhalb der GKV-Leistungspflicht}

Im Rahmen der Untersuchung vor einer ambulanten oder stationären Operationen ist es stets erforderlich, dass der Patient präoperative Befunde zur Operations- bzw. Narkosefähigkeit mit zu seinem Operateur in Praxis oder Krankenhaus mitbringt, wenn diese Untersuchungen nicht direkt von ärztlichen Mitarbeitern des OP-Team/des Krankenhauses ausgeführt werden. Welche Untersuchungen im Einzelfall erforderlich sind, werden Operateur und/oder Narkosearzt festlegen.

In der Regel wird die präoperative Diagnostik vom Hausarzt des Patienten durchgeführte.

Hochrisikopatienten wurden in der folgenden Aufstellung nicht berücksichtigt, da sie in der Regel nicht im Rahmen von IGeL-Leistungen operiert werden.

Wichtig: Aus forensischen Gründen ist eine Operationsaufklärung 24 Stunden vor der Operation erforderlich.

\section{Präoperative Diagnostik im Einzelnen}

Untersuchungsintervalle

- für Röntgenaufnahmen des Thorax maximal drei Monate

- Laboruntersuchungen (Einzelwerte) nicht älter als 6 Wochen

- EKG(Mehrkanal-EKG-Streifen) - und ggf. Lungenfunktionsuntersuchungen (Einzelwerte) sollten bei zwischenzeitlich unauffälliger Anamnese nicht älter als drei Monate alt sein. 
Röntgen-Thorax, ggf. in zwei Ebenen

ist erforderlich bei

- Patientenalter über 60 Jahre

- ASA III/IV (siehe Tabelle)

- anamnestische Hinweise auf kardiopulmonale Störung

- pathologischer Befund bei der körperlichen Untersuchung

\section{EKG - Lungenfunktion}

Grundsätzlich bei allen Patienten ab 40 Jahren, bei jüngeren dann, wenn Hinweise auf eine kardiovaskuläre Erkrankung bestehen.

Eine Lungenfunktionsprüfung ist immer dann erforderlich, wenn auch eine Röntgen-Thoraxaufnahme angefertigt werden muss. Hier ist zunächst die 'kleine Spirometrie' (VC, FEV1, BGA) ausreichend. Bei Patienten mit Lungenerkrankungen und Atemnot ist eine bodyplethysmographische Untersuchung mit Oxymetrie erforderlich.

\section{Laboruntersuchungen}

Bei klinisch gesunden Patienten, kleinen oder mittleren Eingriffen (ggf. auch ambulant) und Operationen, die nicht wegen eines malignen Tumors durchgeführt werden, sind folgende Laboruntersuchungen ausreichend:

- kleines Blutbild inkl. Thrombozyten

- Quick, PTT

- Kalium, Natrium

- Kreatinin und Blutzucker zusätzlich bei Patienten über 60 Jahre

Sind Blutgruppenbestimmung erforderlich, wird der Operateur/Narkosearzt dies vermerken.

In der Regel werden Patienten der ASA-Gruppen* III und IV kaum im Rahmen von IGeL-Leistungen operativ behandelt, sollte dies aber der Fall sein, sind folgende weitere Labor-Untersuchungen erforderlich:

- Blutzucker

- Calcium

- GOT, GPT, -GT, alkalische Phosphatase, LDH, Bilirubin

- Serumeiweiß, Albumin

- Harnstoff, Kreatinin

- Urinstatus, Sediment

\begin{tabular}{|l|l|}
\hline \multicolumn{2}{|l|}{ Die Einteilung in ASA-Gruppen } \\
\hline I & Normaler, gesunder Patient \\
\hline II & Leichte Allgemeinerkrankung ohne Leistungsbeschränkung \\
\hline III & Schwere Allgemeinerkrankung mit Leistungsbeschränkung \\
\hline IV & Schwere Allgemeinerkrankung, die mit oder ohne Operation das Leben des Patienten bedroht \\
\hline V & Moribunder Patient, Tod innerhalb von $24 \mathrm{~h}$ mit oder ohne Operation zu erwarten \\
\hline
\end{tabular}

*American Socienty of Anesthesiologists

\section{Abrechnung praeoperativer Diagnostik}

\begin{tabular}{|c|c|c|c|}
\hline GOÄ Nr. & Kurzlegende & 1 fach $€$ & $\begin{array}{r}{ }^{*} 1,8 / \\
2,3 f a c h \\
\end{array}$ \\
\hline 1 & Beratung, auch tel. & 4,66 & 10,72 \\
\hline 3 & Eingehende Beratung, auch tel. & 8,74 & 20,11 \\
\hline 5 & Symptombezogene Untersuchung & 4,66 & 10,73 \\
\hline 6 & Augen - Gefäßstatus - HNO & 5,83 & 13,41 \\
\hline 7 & $\begin{array}{l}\text { Bauchorgane - Brustorgane - weibl. Genitaltrakt - Haut - } \\
\text { Stütz- u. Bewegungsorgane }\end{array}$ & 9,33 & 21,45 \\
\hline 8 & Ganzkörperstatus & 15,15 & 34,86 \\
\hline $651^{*}$ & EKG & 14,75 & 26,54 \\
\hline $250^{*}$ & Blutentnahme i.v. & 2,55 & 4,20 \\
\hline
\end{tabular}




\begin{tabular}{|c|c|c|c|}
\hline GOÄ-Nr. & Kurzlegende & 1 fach $€$ & $\begin{array}{r}{ }^{*} 1,15 / \\
1,3 \text { fach } €\end{array}$ \\
\hline $3550^{*}$ & Kleines Blutbild & 1,17 & 1,34 \\
\hline $3530^{*}$ & TPZ, Quick & 6,99 & 8,04 \\
\hline $3607^{*}$ & TPZ, Quick & 2,91 & 3,35 \\
\hline $3605^{*}$ & PTT & 2,91 & 3,35 \\
\hline $3557^{*}$ & Kalium & 1,75 & 2,01 \\
\hline $3558^{*}$ & Natrium & 1,75 & 2,01 \\
\hline $3560^{*}$ & Blutzucker & 2,33 & 2,68 \\
\hline 3585.H1* & Kreatinin & 2,33 & 2,68 \\
\hline
\end{tabular}

\section{Reisemedizin}

\section{Reisemedizinische Beratung und Untersuchung}

Nach einer Studie des European Travel Health Advisory Board (2003), wurden 8.000 Reisende, die auf dem Weg in Länder mit niedrigem hygienischen Standard waren, auf Flughäfen in Europa (darunter der Flughafen München), in den USA und im asiatischpazifischen Raum befragt. Das Ergebnis aus Europa zeigt, dass reisemedizinische Beratungen dringend erforderlich sind:

- $48 \%$ der Fernreisenden hatten keine reisemedizinische Beratung erhalten;

- $72 \%$ wussten nicht, dass es in ihren Reiseländern besondere Infektionsrisiken gibt;

- $78 \%$ waren nicht gegen Hepatitis A geimpft;

- $50 \%$ wussten nichts von einem Malaria-Risiko, obwohl sie in ein Malariagebiet reisten. Hier - so schreibt das Blatt sicher sehr berechtigt - gibt es noch viel für Ärzte zu tun!

Mögliche Inhalte einer reisemedizinischen Beratung

- Präventive Maßnahmen mit Hinweisen zur Hygiene und Ernährung im Urlaubsland

- Trinkwasser, Sonnenschutz, Stressminderung

- Umgang mit Gesundheitsrisiken - Hinweise zum Klima im Urlaubsland (Temperaturen), Reiseund Seekrankheiten, Gefahren durch Tiere (z. B. Insekten), Höhenkrankheit, Verhalten bei Flugreisen, sexuell übertragbare Krankheiten und Schutz davor, Hinweise zu Krankheiten im Reiseland, Impfstatus.

\begin{tabular}{|l|l|r|r|}
\hline GOÄ Nr. & Kurzlegende & $\mathbf{1}$ fach $€$ & $\mathbf{2 , 3 f a c h} €$ \\
\hline $\mathbf{3}$ & $\begin{array}{l}\text { Eingehende Beratung (mind. 10 Min.) - nicht neben Sonderleis- } \\
\text { tungen }\end{array}$ & 8,74 & $\mathbf{2 0 , 1 1}$ \\
\hline $\mathbf{1}$ & Kurze Beratung - meist aber GOÄ Nr. 3 erforderlich & 4,66 & $\mathbf{1 0 , 7 3}$ \\
\hline $\mathbf{7 6}$ & Schriftlicher, individueller Ernährungsplan - analoger Ansatz & 4,08 & $\mathbf{9 , 3 8}$ \\
\hline $\mathbf{8}$ & Ganzkörperstatus & 15,15 & $\mathbf{3 4 , 8 5}$ \\
\hline
\end{tabular}

Reisemedizinische Beratungen (abhängig von der Beratungsintensität abgerechnet nach den GOÄ

Nrn. 1 oder 3) sind im Einzelnen sinnvoll zu folgenden Themen:

- Antibabypille: Umstellung/Weiternahme wegen Urlaub

- Beratung besonderer Patientengruppen z. B.

- Ältere Menschen, Kinder, Schwangere

- Beratung von Patientengruppen mit chron. Erkrankungen z. B.

- Allergien

- Asthma bronchiale

- Dialyse-Patienten

- Diabetiker

- Herzkranke

- Impfstatus: allgemeine Impfungen

- Impfstatus: besondere Impfungen für das Reiseland

- Malaria-Prophylaxe

- Reiseapotheke und Selbstmedikation (z. B. Therapie Reise-Diarrhoen, Fieber, Infektionen, Schmerzzustände)

- Sonnenlichtexposition und Hauttyp 
Reiseimpfungen

Schnelle Informationen zu Impfungen sind über die Tropeninstitute zu erhalten

- die Empfehlungen der Ständigen Impfkommission am Robert Koch-Institut (STIKO): www.rki.de/ cln 012/nn 226618/DE/Content/Infekt/Impfen/STIKO Empfehlungen/stiko empfehlungen node. $\mathrm{html} \mathrm{nnn}=$ true

- Forum Impfen: www.forum-impfen.de (neben Impfhinweisen findet man Pat.-Infoblätter, Fachinfos zu den Impfstoffen, Erpertenrat. Für den Zugang muss man sich registrieren lassen; die Mitgliedschaft ist kostenlos).

\begin{tabular}{|l|l|r|r|}
\hline GOÄ Nr. & Kurzlegende & $\mathbf{1 f a c h} €$ & $\mathbf{2 , 3 f a c h ~}$ \\
\hline $\mathbf{3 4}$ & $\begin{array}{l}\text { Erörterung bei Risiken - analog - GOÄ § 6 (2) } \\
\text { GOÄ Kurztext Nr. 34: Erörterung (Dauer mindestens 20 Minuten) } \\
\text { der Auswirkungen einer Krankheit auf die Lebensgestaltung... }\end{array}$ & 17,49 & $\mathbf{4 0 , 2 2}$ \\
\hline $\mathbf{8}$ & Eignungsuntersuchung für Reisen - Ganzkörperstatus & 15,15 & $\mathbf{3 4 , 8 5}$ \\
\hline $\mathbf{3 7 5}$ & Reisemedizinische Impfung & 4,66 & $\mathbf{1 0 , 7 3}$ \\
\hline $\mathbf{3 7 6}$ & Reisemedizinische Impfung: oral & 4,66 & $\mathbf{1 0 , 7 3}$ \\
\hline $\mathbf{3 7 7}$ & Reisemedizinische Impfung: Zusatzimpfung & 2,91 & $\mathbf{6 , 7 0}$ \\
\hline $\mathbf{3 7 8}$ & Simultanimpfung (z. B. aktiv und passiv) & & \\
\hline $\mathbf{7 6}$ & $\begin{array}{l}\text { Schriftliche Zusammenfassung wichtiger reisemedizinischer } \\
\text { Informationen - analog - GOÄ § 6 (2) } \\
\text { GOÄ Kurztext Nr. 76: Schriftlicher Diätplan, individuell... }\end{array}$ & 4,08 & $\mathbf{9 , 3 8}$ \\
\hline $\mathbf{7 7}$ & $\begin{array}{l}\text { Individueller schriftl. Plan zu Prophylaxe und Behandlung, Z. B. } \\
\text { bei komplizierten chron. Begleiterkrankungen oder Reiserisiken - } \\
\text { analog - GOÄ § 6 (2) } \\
\text { GOÄ Kurztext Nr. 77: Schriftliche, individuelle Planung und Leitung einer Kur... }\end{array}$ & 8,74 & $\mathbf{2 0 , 1 1}$ \\
\hline
\end{tabular}

Feststellung der Immunitätslage

Soll auf Patientenwunsch vor einer Fernreise die Immunitätslage festgestellt werden, so ist dies eine IGEL-Leistung und keine Leistung der GKV- oder PKV-Kassen.

\begin{tabular}{|c|c|c|c|}
\hline GOÄ Nr. & Kurzlegende & 1 fach $€$ & 1,15 fach $€$ \\
\hline $4404^{*}$ & Diptherie-AK-IgG & 20,40 & 23,46 \\
\hline 4379* & FSME - IgG & 13,99 & 16,09 \\
\hline $4382^{*}$ & Hepatitis A-Screening - IgG & 13,99 & 16,09 \\
\hline $4400^{*}$ & $\begin{array}{l}\text { Hbs-AK - Hepatitis B - IgG - analog - GOÄ § } 6 \text { (2) } \\
\text { GOÄ Kurztext Nr. 77: Bestimmung von Antikörpern mittels Ligandenassay... }\end{array}$ & 17,49 & 20,11 \\
\hline 4404* & Tetanus - IgG & 20,40 & 23,46 \\
\hline
\end{tabular}

Werden nach einer Reise aufgrund eines Verdachtes auf eine Erkrankung Antikörperbestimmungen und ggf. weitere Untersuchungen erforderlich, so sind dies Leistungen zu Lasten der Krankenkasse.

Reiserücktritt - Ärztliche Bescheinigung

\begin{tabular}{|l|l|r|r|}
\hline GOÄ Nr. & Kurzlegende & $\mathbf{1 f a c h} \boldsymbol{\mathbf { 2 }}$ & $\mathbf{2 , 3 f a c h} \mathbf{}$ \\
\hline $\mathbf{3}$ & $\begin{array}{l}\text { Eingehende Beratung (mind. 10 Min.) -nicht neben Sonderleis- } \\
\text { tungen }\end{array}$ & 8,74 & $\mathbf{2 0 , 1 1}$ \\
\hline $\mathbf{1}$ & Kurze Beratung - meist aber GOÄ Nr. 3 erforderlich & 4,66 & $\mathbf{1 0 , 7 3}$ \\
\hline $\mathbf{5}$ & Symptombezogene Untersuchung - oder ggf. Nr. 7 & 4,66 & $\mathbf{1 0 , 7 2}$ \\
\hline $\mathbf{7}$ & Untersuchung eines Organsystems & 9,33 & $\mathbf{2 1 , 4 5}$ \\
\hline $\mathbf{7 0}$ & Kurze Bescheinigung & 2,33 & $\mathbf{5 , 3 6}$ \\
\hline
\end{tabular}


E. Individuelle Gesundheitsleistungen von A - Z

\section{Sportmedizin}

Sport-Check - Sportmedizinische Vorsorge-Untersuchung

\begin{tabular}{|c|c|c|c|}
\hline GOÄ Nr. & Kurzlegende & 1 fach $€$ & $\begin{array}{r}{ }^{*} 1,8 / \\
2,3 f a c h \\
\end{array}$ \\
\hline 3 & $\begin{array}{l}\text { Eingehende Beratung (mind. } 10 \text { Min.) - nicht neben Sonder- } \\
\text { leistungen }\end{array}$ & 8,74 & 20,11 \\
\hline 1 & Beratung & 4,66 & 10,73 \\
\hline \multicolumn{4}{|c|}{ Die 3 folgenden Untersuchungen GOÄ Nrn. 7,8,29 stehen alternativ zur Verfügung } \\
\hline 7 & Vollständige Untersuchung eines Organsystemes & 9,33 & 21,45 \\
\hline 8 & Ganzkörperstatus: Sportmedizinische Vorsorge-Untersuchung & 15,16 & 34,86 \\
\hline 29 & Gesundheitsuntersuchung & 25,65 & 58,99 \\
\hline $651^{*}$ & EKG in Ruhe & 14,75 & 26,55 \\
\hline 652 & Belastungs-EKG - ggf. mit Lactatbestimmung & 25,94 & 59,66 \\
\hline A 796* & Ergometrische Funktionsprüfung & 8,86 & 15,95 \\
\hline 605 & Lungenfunktion & 14,11 & 32,45 \\
\hline $605 a$ & Flussvolumenkurve & 8,16 & 14,69 \\
\hline $606^{*}$ & Spiroergometrische Untersuchung & 22,09 & 39,76 \\
\hline 715 & $\begin{array}{l}\text { Prüfung der Entwicklung - analog - GOÄ § } 6 \text { (2) } \\
\text { GOÄ Kurztext Nr. 715: Prüfung der kindlichen Entwicklung }\end{array}$ & 12,82 & 29,49 \\
\hline \multirow{2}{*}{$250^{*}$} & Blutentnahme & 2,33 & 5,36 \\
\hline & Labor & 1 fach & 1,15 fach $€$ \\
\hline $3511^{*}$ & $\begin{array}{l}\text { Untersuchung eines Körpermaterial (z. B. Glukose, Harnstoff, } \\
\text { Urinteststreifen) }\end{array}$ & 2,91 & 3,35 \\
\hline $3550^{*}$ & Blutbild & 3,50 & 4,02 \\
\hline $3551^{*}$ & Differentialblutbild & 1,17 & 1,34 \\
\hline $3560^{*}$ & Glukose & 2,33 & 2,68 \\
\hline 3562.H1* & Cholesterin & 2,33 & 2,68 \\
\hline 3563.H1* & HDL-Cholesterin & 2,33 & 2,68 \\
\hline 3585.H1* & Kreatinin & 2,33 & 2,68 \\
\hline 3592.H1* & Gamma-GT & 2,33 & 2,68 \\
\hline 3595.H1* & GPT & 2,33 & 2,68 \\
\hline $3511^{*}$ & Urin-Teststreifen & 2,91 & 3,35 \\
\hline $3781^{*}$ & Lactatbestimmung zur Einschätzung der Ausdauerleistung & 12,82 & 14,75 \\
\hline
\end{tabular}

Sportbootführerschein - Eignungsuntersuchung

\begin{tabular}{|l|l|r|r|}
\hline GOÄ Nr. & Kurzlegende & $\mathbf{1}$ fach $€$ & $\mathbf{2 , 3 f a c h} €$ \\
\hline $\mathbf{1}$ & Kurze Beratung & 4,66 & $\mathbf{1 0 , 7 3}$ \\
\hline $\mathbf{8}$ & Ganzkörperstatus & 15,15 & $\mathbf{3 4 , 8 6}$ \\
\hline $\mathbf{1 2 0 1}$ & Subj. Refraktionsbestimmung mit. sphärischen-zylindr. Gläsern & 5,19 & $\mathbf{1 1 , 9 3}$ \\
\hline $\mathbf{1 2 2 8}$ & Farbsinnprüfung mit Pigmentproben (Farbtafeln) & 3,56 & $\mathbf{8 , 1 8}$ \\
\hline $\mathbf{7 0}$ & Kurze Bescheinigung & 2,33 & $\mathbf{5 , 3 6}$ \\
\hline
\end{tabular}

Flugtauchlichkeitsuntersuchung

\begin{tabular}{|l|l|r|r|}
\hline GOÄ Nr. & Kurzlegende & $\mathbf{1 f a c h ~} €$ & $\begin{array}{r}{ }^{\star 1,8 /} \\
\mathbf{2 , 3 f a c h} €\end{array}$ \\
\hline $\mathbf{3}$ & $\begin{array}{l}\text { Eingehende Beratung (mind. 10 Min.) - nicht neben Sonder- } \\
\text { leistungen }\end{array}$ & 8,74 & $\mathbf{2 0 , 1 1}$ \\
\hline
\end{tabular}




\begin{tabular}{|c|c|c|c|}
\hline GOÄ Nr. & Kurzlegende & 1fach $€$ & $\begin{array}{r}{ }^{*} 1,8 / \\
2,3 \text { fach } €\end{array}$ \\
\hline 8 & Eignungsuntersuchung für Flugtauglichkeit & 15,16 & 34,86 \\
\hline 70 & Kurze Bescheinigung & 2,33 & 5,36 \\
\hline 652 & EKG in Ruhe und Belastung & 25,94 & 59,66 \\
\hline $250^{*}$ & Blutabnahme aus der Vene & 2,33 & 4,19 \\
\hline 605 & Ruhespirographische Untersuchung & 14,11 & 32,45 \\
\hline $605 a$ & Flussvolumenkurve & 8,16 & 18,77 \\
\hline \multirow[t]{2}{*}{1401} & Hörtest & 3,50 & 6,30 \\
\hline & Labor & 1 fach & 1,15 fach $€$ \\
\hline $3511^{*}$ & Urinstreifentest & 2,91 & 3,35 \\
\hline $3550^{*}$ & Blutbild, klein & 3,15 & 4,03 \\
\hline $3560^{*}$ & Blutzucker & 2,33 & 2,68 \\
\hline $3583 \mathrm{H}^{*}$ & Harnsäure & 2,33 & 2,68 \\
\hline $3592 \mathrm{H}^{*}{ }^{*}$ & Gamma-GT & 2,33 & 2,68 \\
\hline $3885 \mathrm{H}^{*}$ & Kreatinin & 2,33 & 2,68 \\
\hline
\end{tabular}

Tauchsport-Untersuchung

\begin{tabular}{|c|c|c|c|}
\hline GOÄ Nr. & Kurzlegende & 1 fach $€$ & $\begin{array}{r}{ }^{*} 1,8 / \\
2,3 f a c h €\end{array}$ \\
\hline 3 & $\begin{array}{l}\text { Eingehende Beratung (mind. } 10 \text { Min.) - nicht neben Sonder- } \\
\text { leistungen }\end{array}$ & 8,74 & 20,11 \\
\hline 8 & Eignungsuntersuchung für Tauchsport & 15,16 & 34,86 \\
\hline 70 & Kurze Bescheinigung & 2,33 & 5,36 \\
\hline $651^{*}$ & EKG & 14,75 & 26,54 \\
\hline 652 & EKG in Ruhe und Belastung & 25,94 & 59,66 \\
\hline 605 & Ruhespirographische Untersuchung & 14,11 & 32,45 \\
\hline $605 a$ & Flussvolumenkurve & 8,16 & 18,77 \\
\hline \multirow[t]{2}{*}{$250^{*}$} & Blutabnahme aus der Vene & 2,33 & 4,19 \\
\hline & Labor & 1 fach $€$ & 1,15 fach $€$ \\
\hline $3511^{*}$ & Urin-Teststreifen & 2,91 & 3,35 \\
\hline
\end{tabular}




\section{Augenheilkunde}

Was bietet die Fachgruppe der Augenärzte als IGeL-Leistungen an?

Die Autoren haben zahlreiche Internetseiten von niedergelassenen Augenärzten und einzelnen Augenkliniken auf IGeL-Angebote untersucht. Die folgende Aufstellung gibt die am häufigsten den Patienten angebotenen Leistungen - in der Diktion der Ärzte im Web - wieder.

Der Patient findet auch Leistungen, die primär nicht ins Gebiet der Augenheilkunde fallen, aber vom jeweiligen Augenarzt angeboten werden:

- Akupunktur

- Akupunkturtaping

- Arbeitsbrillenanpassung

- Arbeitsmedizinische Untersuchung z. B. auf Veranlassung des Arbeitgebers

- Beratung vor Auslandsaufenthalten

- Besenreiser etc. - Gefäßverödung mit Argonlaser

- Biometrie (IOL Master), Kalkulation der Kunstlinsenstärke

- Untersuchung zur Verordnung einer Bildschirmbrille (außerhalb arbeitsmedizinischer Untersuchungen)

- Brillenberatung

- Untersuchung des Farbunterscheidungsvermögens

- Fahrgutachten

- Fliegerärztliche Untersuchungen

- Führerschein-Sehtest, -Zeugnis, -Gutachten

- Glaukomvorsorge: Messung der Sehnervenfasern (GDx)

- Früherkennung Grüner Star (Glaukom)

- Homöopathie

- Hornhaut-Dickenmessung (Pachymetrie)

- Hornhauttopographie

- HRT (Heidelberger-Retina-Tomograph) Vermessung der Papille, Nervenfaseranalyse

- Intravitreale Injektionen bei feuchter Makuladegeneration (z. B. Avastin, Lucentis oder Macugen)

- Kinder-Augenvorsorge (Amblyopie)

- Kontaktlinsen Anpassung/Beratung außerhalb der Erstattungsfähigkeit durch die Krankenkassen

- Lidoperationen:

- Korrekturen der Ober- und Unterlider aus kosmetischen Gründen z. B. Schlupflider

- Entfernung störender Hautveränderungen

- Laser-Chirurgie z. B. Entfernung von Warzen und Muttermalen

- altersbezogene Makuladegeneration (AMD) - Vorsorgeuntersuchungen

- Untersuchung des Nachtsehens, der Blendungsempfindlichkeit und der Nachtfahrtauglichkeit

- Optos-Netzhautuntersuchung zur Früherkennung von Augenerkrankungen

- Perimetrie - Bestimmung des Gesichtsfeldes

- Photodynamische Therapie (PDT) von chorioidalen Neovaskularisationen (CNV) im Makulabereich

- Refraktive Chirurgie, folgende Verfahren:

- LASIK (Laser-in-situ-Keratomileusis)

- PRK (Photorefraktive Keratektomie)

- PKT (Phototherapeutische Keratektomie)

- LASEK (Laserassistierte epitheliale Keratomileusis)

- Sauerstoff-Mehrschnitt-Therapie

- Schlaganfall-Vorsorge (ARIC- Atherosclerosis Risk in Communities) Bestimmung des Schlaganfall-Risikoprofils

- Sehfehlerkorrektur mit Laser

- Sportmedizinische augenärztliche Untersuchungen

- Stereoskopische Vermessung des Sehnerven bei Patienten mit Glaukom

- Tränenfilmanalyse (Tearscope)

- Xanthelasmenbehandlung mit Hochfrequenzchirurgische oder Laser

Ohne Wertung - aber doch mit Verwunderung - werden die folgenden Angebote aus Praxis-Internetseiten einiger Augenärzten aufgeführt: 


\section{Alternativtherapie}

Maculadegeneration

- Sauerstofftherapie, Nahrungsergänzung: mit Vitaminen und Antioxidantien

- Akupunktur zur Wiederherstellung des Energiegewichtes

Glaucom (Grüner Star)

- Akupunktur zur Wiederherstellung des Energiegewichts, Sauerstofftherapie, Nahrungsergänzung mit Vitaminen und Antioxidation, Körperliche Aktivität: regelmäßiger Sport, insbesondere Ausdauersport, Entspannungstraining

Complementäre und Alternative Medizin (CAM) hilft bei:

- Maculadegeneration

- Thrombose/Verschlusserkrankungen

- Glaukom

- Kurzsichtigkeit/Myopie

- Allergien

- Schmerztherapie/Kopfschmerzen

- Entwöhnung, Nikotin, Essstörung

- Tinnitus - „Schwindel“

\section{Magnetfeldtherapie}

- bei Durchblutungsstörungen, Schmerzzuständen und Wundheilungs- störungen.

Früherkennung von Schwachsichtigkeit und Schielen im Kleinkind- und Vorschulalter

Die augenärztliche Untersuchung auf Amblyopie und Strabismus umfasst

- Inspektion der Augen und der Adnexe

- Prüfung der Augenstellung und der Beweglichkeit, dabei sind Ab- und Aufdeck-Test und BrücknerTest anzuwenden.

- Bestimmung der Sehschärfe entsprechend dem Alter

- Objektive Refraktionsbestimmung in Zykloplegie (Skiaskopie oder Refraktometer)

- Betrachtung des zentralen Augenhintergrundes

- Prüfung der sensorischen Binokularfunktion mit Stereopsis (z. B. Lang-Titmous-, TNO-Test)

\begin{tabular}{|l|l|r|r|}
\hline GOÄ Nr. & Kurzlegende & $\mathbf{1 f a c h} €$ & $\mathbf{2 , 3 f a c h} €$ \\
\hline $\mathbf{3}$ & $\begin{array}{l}\text { Eingehende Beratung (mind. 10 Min.) - nicht neben Sonderleis- } \\
\text { tungen }\end{array}$ & 8,74 & $\mathbf{2 0 , 1 1}$ \\
\hline $\mathbf{6}$ & Untersuchung Augen & 5,83 & $\mathbf{1 3 , 4 1}$ \\
\hline $\mathbf{1 2 0 0}$ & Subj. Refraktionsbestimmung mit sphärischen Gläsern & 3,44 & $\mathbf{7 , 9 1}$ \\
\hline $\mathbf{1 2 0 1}$ & Subj. Refraktionsbestimmung mit. sphärischen-zylindr. Gläsern & 5,19 & $\mathbf{1 1 , 9 3}$ \\
\hline $\mathbf{1 2 0 2}$ & Objekt. Refraktionsbestimmung & 4,32 & $\mathbf{9 , 9 2}$ \\
\hline $\mathbf{1 2 1 6}$ & Untersuchung auf Heterophorie bzw. Strabismus & 5,30 & $\mathbf{1 2 , 2 0}$ \\
\hline
\end{tabular}

Führerschein-Sehtest

\begin{tabular}{|l|l|r|r|}
\hline GOÄ Nr. & Kurzlegende & $\mathbf{1}$ fach $€$ & $\mathbf{2 , 3 f a c h} €$ \\
\hline $\mathbf{1}$ & Beratung & 4,66 & $\mathbf{1 0 , 7 3}$ \\
\hline $\mathbf{1 2 0 0}$ & Refraktionsbestimmung, subjektive & 3.44 & $\mathbf{7 , 9 1}$ \\
\hline $\mathbf{7 0}$ & Kurze Bescheinigung oder kurzes Zeugnis & 2,33 & $\mathbf{5 , 3 6}$ \\
\hline
\end{tabular}

Die Fahrerlaubnis-Verordnung (FeV) - Anlage 6 (zu §§ 12, 48 Abs. 4 und 5) schreibt vor, dass wenn der Bewerber den Sehtest nicht besteht, eine augenärztliche Untersuchung erforderlich ist.

Augenärztliche Untersuchung/Gutachten zu Führerscheinen der Klassen C1, C, C1E, CE, D

\begin{tabular}{|l|l|r|r|}
\hline GOÄ Nr. & Kurzlegende & $\mathbf{1 f a c h} €$ & $\begin{array}{r}{ }^{* 1,8 /} \\
\mathbf{2 , 3 f a c h} €\end{array}$ \\
\hline $\mathbf{3}$ & $\begin{array}{l}\text { Eingehende Beratung (mind. 10 Min.) - nicht neben Sonderleis- } \\
\text { tungen }\end{array}$ & 8,74 & $\mathbf{2 0 , 1 1}$ \\
\hline $\mathbf{1}$ & Beratung & 4,66 & $\mathbf{1 0 , 7 3}$ \\
\hline
\end{tabular}


E. Individuelle Gesundheitsleistungen von A - Z

\begin{tabular}{|l|l|r|r|}
\hline GOÄ Nr. & Kurzlegende & $\mathbf{1 f a c h} €$ & $\begin{array}{r}{ }^{*} \mathbf{1 , 8 /} \\
\mathbf{2 , 3 f a c h} \boldsymbol{\text { f }}\end{array}$ \\
\hline $\mathbf{1 2 0 0}$ & Refraktionsbestimmung, subjektive & 3.44 & $\mathbf{7 . 9 1}$ \\
\hline $\mathbf{1 2 1 6}$ & Untersuchung auf Heterophorie & 5,30 & $\mathbf{1 2 , 2 0}$ \\
\hline $\mathbf{1 2 2 6}$ & Projektionsperimetrie & 10,61 & $\mathbf{2 4 , 4 0}$ \\
\hline $\mathbf{1 2 2 8}$ & Farbsinnprüfung mit Pigmentproben & 3,56 & $\mathbf{8 , 1 8}$ \\
\hline $\mathbf{1 2 4 0}$ & Spaltlampenmikroskopie & 4,31 & $\mathbf{9 , 9 2}$ \\
\hline $\mathbf{8 0}$ & Schriftl. Gutachterliche Äußerung & 17,49 & $\mathbf{4 0 , 2 2}$ \\
\hline $\mathbf{8 5}$ & Gutachten, erhöhter Aufwand & 29,14 & $\mathbf{6 7 , 0 2}$ \\
\hline $\mathbf{9 5}$ & Schreibgebühr je angefangene DIN A Seite & $\mathbf{3 , 5 0}$ &,-- \\
\hline $\mathbf{9 6}$ & Kopiergebühr, je Kopie & $\mathbf{0 , 1 7}$ &,-- \\
\hline
\end{tabular}

Glaukomfrüherkennung

\begin{tabular}{|l|l|r|r|}
\hline GOÄ Nr. & Kurzlegende & $\mathbf{1 f a c h} \boldsymbol{€}$ & $\begin{array}{r}{ }^{*} \mathbf{1 , 8} / \\
\mathbf{2 , 3 f a c h} \boldsymbol{\ell}\end{array}$ \\
\hline $\mathbf{3}$ & $\begin{array}{l}\text { Eingehende Beratung (mind. 10 Min.) - nicht neben Sonderleis- } \\
\text { tungen }\end{array}$ & 8,74 & $\mathbf{2 0 , 1 1}$ \\
\hline $\mathbf{6}$ & Untersuchung Augen & 5,83 & $\mathbf{1 3 , 4 1}$ \\
\hline $\mathbf{1 2 4 0}$ & Spaltlampenmikroskopie & 4,31 & $\mathbf{9 , 9 2}$ \\
\hline $\mathbf{1 2 5 6}^{*}$ & Tonometrie & 5,83 & $\mathbf{1 0 , 4 9}$ \\
\hline
\end{tabular}

Kontaktlinsen-Anpassung

\begin{tabular}{|l|l|r|r|}
\hline GOÄ Nr. & Kurzlegende & $\mathbf{1 f a c h} €$ & $\mathbf{2 , 3 f a c h} \mathbf{~}$ \\
\hline $\mathbf{3}$ & $\begin{array}{l}\text { Eingehende Beratung (mind. 10 Min.) - nicht neben Sonderleis- } \\
\text { tungen }\end{array}$ & 8,74 & $\mathbf{2 0 , 1 1}$ \\
\hline $\mathbf{1 2 1 0}$ & $\begin{array}{l}\text { Kontaktlinsen-Anpassung und -Kontrolle ohne GKV-Indikation } \\
\text { zur Kontaktlinsen-Versorgung (ein Auge) }\end{array}$ & 13,29 & $\mathbf{3 0 , 5 7}$ \\
\hline $\mathbf{1 2 1 1}$ & Kontaklinsen-Versorgung (beide Augen) & 17,49 & $\mathbf{4 0 , 2 2}$ \\
\hline $\mathbf{1 2 1 2}$ & Prüfung auf Sitz und Funktion der Kontaktlinsen - ein Auge & 7,69 & $\mathbf{1 7 , 7 0}$ \\
\hline $\mathbf{1 2 1 3}$ & Prüfung auf Sitz und Funktion der Kontaktlinsen - beide Augen & 11,54 & $\mathbf{2 6 , 5 4}$ \\
\hline
\end{tabular}

Ausschlüsse: Nr. 6 nicht neben 103, 1204, 1210-1213, 1228, 1240

Augenärztliche Untersuchung nach der Verordnung über Sicherheit und Gesundheitsschutz bei der Arbeit an Bildschirmgeräten

siehe http://bundesrecht.juris.de/bildscharbv/

\section{§ 6 Untersuchung der Augen und des Sehvermögens}

(1) Der Arbeitgeber hat den Beschäftigten vor Aufnahme ihrer Tätigkeit an Bildschirmgeräten, anschließend in regelmäßigen Zeitabständen sowie bei Auftreten von Sehbeschwerden, die auf die Arbeit am Bildschirmgerät zurückgeführt werden können, eine angemessene Untersuchung der Augen und des Sehvermögens durch eine fachkundige Person anzubieten. Erweist sich aufgrund der Ergebnisse einer Untersuchung nach Satz 1 eine augenärztliche Untersuchung als erforderlich, ist diese zu ermöglichen.

(2) Den Beschäftigten sind im erforderlichen Umfang spezielle Sehhilfen für ihre Arbeit an Bildschirmgeräten zur Verfügung zu stellen, wenn die Ergebnisse einer Untersuchung nach $A b$ satz 1 ergeben, daß spezielle Sehhilfen notwendig und normale Sehhilfen nicht geeignet sind.

\begin{tabular}{|l|l|r|r|}
\hline GOÄ Nr. & Kurzlegende & $\mathbf{1 f a c h} €$ & $\mathbf{2 , 3 f a c h} €$ \\
\hline $\mathbf{1}$ & Beratung & 4,66 & $\mathbf{1 0 , 7 3}$ \\
\hline $\mathbf{6}$ & Untersuchung Augen & 5,83 & $\mathbf{1 3 , 4 1}$ \\
\hline
\end{tabular}




\begin{tabular}{|l|l|r|r|}
\hline GOÄ Nr. & Kurzlegende & $\mathbf{1 f a c h} €$ & $\mathbf{2 , 3 f a c h} €$ \\
\hline $\mathbf{1 2 0 1}$ & Subj. Refraktionsbestimmung mit. sphärischen-zylindr. Gläsern & 5,19 & $\mathbf{1 1 , 9 3}$ \\
\hline $\mathbf{1 2 0 3}$ & Messung der Maximal- oder Gebrauchsakkommodation & 3,50 & $\mathbf{8 , 0 4}$ \\
\hline $\mathbf{1 2 1 6}$ & Untersuchung auf Heterophorie bzw. Strabismus & 5,30 & $\mathbf{1 2 , 2 0}$ \\
\hline $\mathbf{1 2 2 5}$ & Kampimetrie - auch Perimetrie & 7,05 & $\mathbf{1 6 , 2 2}$ \\
\hline $\mathbf{7 0}$ & Kurze Bescheinigung oder kurzes Zeugnis & 2,33 & $\mathbf{5 , 3 6}$ \\
\hline
\end{tabular}

Immer häufiger werden von Augenärzten zur Fehlsichtigkeitskorrektur (schwache und mittelstarke Fehlsichtigkeiten) Behandlungen mit dem Excimerlaser (LASIK-; LASEK- und PRK-Technik) angeboten.

\section{Augenärztliche Operationen}

Blepharochalasis-OP

\begin{tabular}{|l|l|r|r|}
\hline GOÄ Nr. & Kurzlegende & $\mathbf{1 f a c h} €$ & $\mathbf{2 , 3 f a c h} €$ \\
\hline $\mathbf{3}$ & $\begin{array}{l}\text { Eingehende Beratung (mind. 10 Min.) - nicht neben Sonderleis- } \\
\text { tungen }\end{array}$ & 8,74 & $\mathbf{2 0 , 1 1}$ \\
\hline $\mathbf{1}$ & Beratung & 4,66 & $\mathbf{1 0 , 7 3}$ \\
\hline $\mathbf{4 9 1}$ & Infiltrationsanästhesie gr. Bezirke & 7,05 & $\mathbf{1 6 , 2 2}$ \\
\hline $\mathbf{1 3 1 1}$ & Augenlidplastik mittels Hautverschiebung & 64,70 & $\mathbf{1 4 8 , 8 1}$ \\
\hline $\mathbf{2 4 0 4}$ & Exzision einer Fettgeschwulst & 32,29 & $\mathbf{7 4 , 2 7}$ \\
\hline $\mathbf{4 4 3}$ & $\begin{array}{l}\text { Zuschlag zu Nr. 2404 bei amb. OP - nur 1-facher Satz abrechen- } \\
\text { ba }\end{array}$ & $\mathbf{7 5 , 7 7}$ & - \\
\hline $\mathbf{4 4 1}$ & Zuschlag zu Nr. 2404 bei amb. OP für Laser & $\mathbf{6 4 , 7 0}$ & - \\
\hline
\end{tabular}

Entfernung eines Lidtumors

\begin{tabular}{|l|l|r|r|}
\hline GOÄ Nr. & Kurzlegende & $\mathbf{1 f a c h} €$ & $\mathbf{2 , 3 f a c h} €$ \\
\hline $\mathbf{3}$ & $\begin{array}{l}\text { Eingehende Beratung (mind. 10 Min.) - nicht neben Sonderleis- } \\
\text { tungen }\end{array}$ & 8,74 & $\mathbf{2 0 , 1 1}$ \\
\hline $\mathbf{1}$ & Beratung & 4,66 & $\mathbf{1 0 , 7 3}$ \\
\hline $\mathbf{4 9 1}$ & Infiltrationsanästhesie gr. Bezirke & 7,05 & $\mathbf{1 6 , 2 2}$ \\
\hline $\mathbf{1 2 8 2}$ & Entfernung einer Geschwulst & 8,86 & $\mathbf{2 0 , 3 8}$ \\
\hline $\mathbf{2 0 4}$ & Kompressionsverband & 5,54 & $\mathbf{1 2 , 7 4}$ \\
\hline
\end{tabular}

Zur kosmetische Lidchirurgie gehört die Behandlung von:

- Blepharochalasis (Schlupflider) - Chalazion (Hagelkorn)

- Kosmetische Lidstraffung - Gutartige Lidtumoren

- Ptosis

- Xanthelasma

Refraktionschirurgie auf Patientenwunsch

1. Präoperative Diagnostik vor Refraktionschirurgie*

\begin{tabular}{|l|l|r|r|}
\hline GOÄ Nr. & Kurzlegende & $\mathbf{1 f a c h} €$ & $\begin{array}{r}{ }^{* 1,8 /} \\
\mathbf{2 , 3 f a c h} €\end{array}$ \\
\hline $\mathbf{3}$ & $\begin{array}{l}\text { Eingehende Beratung (mind. 10 Min.) - nicht neben Sonderleis- } \\
\text { tungen }\end{array}$ & 8,74 & $\mathbf{2 0 , 1 1}$ \\
\hline $\mathbf{1}$ & Beratung & 4,66 & $\mathbf{1 0 , 7 3}$ \\
\hline $\mathbf{6}$ & Untersuchung aller Augenabschnitte & 5,83 & $\mathbf{1 3 , 4 1}$ \\
\hline $\mathbf{1 2 0 1}$ & Subj. Refraktionsbestimmung mit. sphärischen-zylindr. Gläsern & 5,19 & $\mathbf{1 1 , 9 3}$ \\
\hline $\mathbf{1 2 0 2}$ & Objekt. Refraktionsbestimmung & 4,32 & $\mathbf{9 , 9 2}$ \\
\hline
\end{tabular}


E. Individuelle Gesundheitsleistungen von A - Z

\begin{tabular}{|l|l|r|r|}
\hline GOÄ Nr. & Kurzlegende & $\mathbf{1}$ fach $\boldsymbol{\ell}$ & $\begin{array}{r}{ }^{*} \mathbf{1 , 8} / \\
\mathbf{2 , 3 f a c h} \boldsymbol{\ell}\end{array}$ \\
\hline $\mathbf{1 2 0 9}$ & Nachweis der Tänensekretionsmenge & 1,17 & $\mathbf{2 , 6 8}$ \\
\hline $\mathbf{1 2 1 6}$ & Untersuchung auf Heterophorie bzw. Strabismus & 5,30 & $\mathbf{1 2 , 2 0}$ \\
\hline $\mathbf{1 2 3 4}$ & Untersuchung des Dämmerungssehen ohne Blendung & 5,30 & $\mathbf{1 2 , 2 0}$ \\
\hline $\mathbf{1 2 3 5}$ & Untersuchung des Dämmerungssehen während der Blendung & 5,30 & $\mathbf{1 2 , 2 0}$ \\
\hline $\mathbf{1 2 4 2}$ & Biokulare Untersuchung des Augenhintergrundes & 8,86 & $\mathbf{2 0 , 3 7}$ \\
\hline $\mathbf{1 2 5 6}^{*}$ & Tonometrie & 5,83 & $\mathbf{1 0 , 4 9}$ \\
\hline
\end{tabular}

* In Einzelfällen wird die präoperative Diagnostik erweitert

\section{Refraktionschirurgie}

\begin{tabular}{|c|c|c|c|}
\hline GOÄ Nr. & Kurzlegende & 1 fach $€$ & 2,3 fach $€$ \\
\hline 3 & $\begin{array}{l}\text { Eingehende Beratung (mind. } 10 \text { Min.) - nicht neben Sonderleis- } \\
\text { tungen }\end{array}$ & 8,74 & 20,11 \\
\hline 1 & Beratung & 4,66 & 10,73 \\
\hline 1345 & $\begin{array}{l}\text { Laser in situ - Keratomileusis (Lasik) } \\
\text { mit Excimer-Laseranwendung - analoger Ansatz - GOÄ § } 6 \text { (2) } \\
\text { GOÄ Kurztext Nr. 1345: Hornhautplastik }\end{array}$ & 96,76 & 222,54 \\
\hline $5855^{\star} A$ & $\begin{array}{l}\text { Photorefraktäre Keratektomie (PRK) } \\
\text { mit Excimer-Laseranwendung - analog Ansatz - GOÄ § } 6 \text { (2) } \\
\text { Empfehlung der BÄK - GOÄ Kurztext Nr. 5855: Intraoperative Strahlenbehand- } \\
\text { lung mit Elektronen }\end{array}$ & 402,18 & 723,93 \\
\hline
\end{tabular}

Hinweise zur Abrechnung bei der BÄK: www.bundesaerztekammer.de/30/Gebuehrenordnung/40 Abrechnung/40Beschluesse/Augen/Refraktionschir.html

\section{Postoperatative Kontrolle nach Refraktionschirurgie}

\begin{tabular}{|l|l|r|r|}
\hline GOÄ Nr. & Kurzlegende & $\mathbf{1 f a c h} €$ & $\mathbf{2 , 3 f a c h ~} \mathbf{~}$ \\
\hline $\mathbf{3}$ & $\begin{array}{l}\text { Eingehende Beratung (mind. 10 Min.) - nicht neben Sonderleis- } \\
\text { tungen }\end{array}$ & 8,74 & $\mathbf{2 0 , 1 1}$ \\
\hline $\mathbf{1}$ & Beratung & 4,66 & $\mathbf{1 0 , 7 3}$ \\
\hline $\mathbf{1 2 0 1}$ & Subj. Refraktionsbestimmung mit. sphärischen-zylindr. Gläsern & 5,19 & $\mathbf{1 1 , 9 3}$ \\
\hline $\mathbf{1 2 4 0}$ & Spaltlampenmikroskopie & 4,31 & $\mathbf{9 , 9 2}$ \\
\hline
\end{tabular}


E. Individuelle Gesundheitsleistungen von A-Z

\section{Chirurgie - Plastische Chirurgie}

Die operativen Angebote von einzelnen Praxen vor allem der Dermatologie, HNO, Gynäkologie und Urologie sind durchaus sehr spezialisiert und dadurch umfangreich.

Hinweise zu IGeL-Operationen aus dem Bereich der Plastischen Chirurgie sind im Buch bei den entsprechenden Fachgebieten, die ambulanten Operationen anbieten, aufgeführt (z. B. Dermatologie, Gynäkologie, HNO, Urologie).

Die folgende Aufstellung zeigt, dass auch Uni-Kliniken inzwischen grosses Interesse am IGeLn haben.

\section{Was bietet die Fachgruppe an IGeL-Leistungen?}

Die Abt. Plastische und Handchirurgie des Universitätsklinikums Freiburg bietet Patienten unter www.uniklinik-freiburg.de/ip/live/patientenservice/zusatzangebote/plastchir.html mehrere Operationen an:

.... „Der Wunsch nach Schönheit ist für die Abteilung Plastische und Handchirurgie des Universitätsklinikums Freiburg kein Tabu. Unser Anliegen ist das Verständnis für die subjektive Empfindung des Erscheinungsbildes unserer Patienten.

Als eine von wenigen eigenständigen Abteilungen für Plastische Chirurgie an einer deutschen Universitätsklinik bieten wir Ihnen im Rahmen von uniklinik plus auch außerhalb der Privatsprechstunde ein breites Spektrum plastisch chirurgischer Eingriffe durch erfahrene Operateure an. Dazu zählen Facelifting und Nasenkorrekturen, Korrekturen an Augenlidern und Ohren, Brustoperationen, Fettabsaugungen, Straffungsoperationen und Laserbehandlung von Fältchen. Unsere Fachärztinnen und Fachärzte für Plastische Chirurgie beraten Sie dabei individuell und objektiv zu allen Eingriffen.

\section{Nasenkorrekturen und Kinnvergrößerung}

Nase und Kinn sind für die harmonische Wirkung des menschlichen Gesichts und des Profils von entscheidender Bedeutung. Über Ihre individuellen Möglichkeiten einer Korrektur von Nasen- oder Kinnform beraten wir Sie gerne.

\section{Korrekturen Ober- und Unterlider}

Lidkorrekturen werden im allgemeinen in örtlicher Betäubung durchgeführt. Individuell muss hierfür das richtige Verfahren ausgewählt werden. An den Unterlidern wird auf äußere Schnitte ganz verzichtet und durch den Bindehautsack operiert.

\section{Ohrenkorrekturen}

Bei der Ohrkorrektur werden meistens abstehende Ohren angelegt oder besonders große Ohren verkleinert. Besonders große Ohrläppchen können ebenfalls verkleinert werden.

\section{Facelifting}

Zur nachhaltigen Behandlung von Alterserscheinungen des Gesichts und des Halses wenden wir auf den Einzelfall abgestimmte, auch endoskopisch durchgeführte Operationsmethoden an.

\section{Faltenbehandlung}

Kleine Fältchen, insbesondere im Stirn und Augenlidbereich (sog. Zornesfalten oder Krähenfüße) können durch Botox-Injektionen verbessert werden, ggf. muß im Abstand von einigen Monaten wiederholt eingespritzt werden. Im Lippenbereich oder an anderen Stellen im Gesicht kann eine Auffüllung der Falten mit Eigenfett erreicht werden. Dabei wird schonend Fett vom Bauch abgesaugt und das aufbereitete Fett in die Falten eingespritzt. Die Faltenbehandlung mit Hyaluronsäure wird ebenfalls angeboten.

\section{Bauchdeckenstraffung}

Nach einer starken, länger bestehenden Ausdehnung der Bauchdecke kommt es bei Frauen und Männern zu einem Auseinanderweichen der Bauchmuskulatur, die durch Diät oder Gymnastik nicht mehr zu beheben ist. Mit der Bauchdeckenstraffung steht eine gute Möglichkeit zur Verfügung, das als unschön empfundene Aussehen der Bauchdecke zu verbessern.

\section{Fettabsaugung}

Hartnäckige, weder einer Reduktionsdiät noch gezielter Gymnastik zugängliche Fettpolster kommen bei vielen Frauen und Männern vor. Mit einer gezielten, sorgfältigen Ultraschall-Absaugung des Unterhautfetts kann diese Problematik verbessert oder behoben werden. 


\section{Oberschenkelstraffung}

Liegt nicht nur ein Fettüberschuss sondern eine erhebliche Hauterschlaffung vor, kann eine Oberschenkelstraffung erforderlich sein.

\section{Ratenzahlung für Schönheitsoperationen möglich!}

Im Internet wird von der Praxis-Klinik für Plastische und Ästhetische Chirurgie: KÖ - AESTHETICS DÜSSELDORF (http://www.koe-aesthetics.de/finanzierung.html?L=\%252523navigation-main9 Ratenzahlung für Schönheitschirurgie mit folgendem Text (Ausschnitt) angeboten:

\section{Ratenzahlung - Ein Service der KÖ-AESTHETICS}

.... „Die Kosten für eine Behandlung sind ohne vorherige Untersuchung pauschal nicht vorhersagbar. Jede Behandlung ist individuell, so dass die Behandlungskosten auch individuell unterschiedlich sind.

Wir können Ihnen weder am Telefon noch per Mail die Kosten und Preise Ihrer Schönheitsoperation nennen.

Die Kosten sind abhängig von OP-Dauer, Implantaten, OP-Technik, Lifting oder Saugung, stationär oder ambulant und vielen anderen Faktoren.

Im Rahmen eines ausführlichen Beratungsgesprächs können wir Ihnen einen detaillierten Kostenvoranschlag erstellen.

Wir bieten für jede Behandlung eine Finanzierung an, die über medipay abgewickelt werden kann..."

\section{Schönheits-OP Finanzierung}

Folgende Operationen können zum Beispiel finanziert (siehe Ratenzahlungs-Tabelle) werden:

- Brust OP Finanzierung/Ratenzahlung

- Brustvergrößerung Finanzierung/Ratenzahlung

- Bruststraffung Finanzierung/Ratenzahlung

- Fettabsaugung Finanzierung/Ratenzahlung

- Nasenkorrektur Finanzierung/Ratenzahlung

- Bauchdeckenstraffung Finanzierung/Ratenzahlung

- Facelift Finanzierung/Ratenzahlung

- Gynäkomastie Finanzierung/Ratenzahlung

- Lidstraffung Finanzierung/Ratenzahlung

- Faltenbehandlung Finanzierung/Ratenzahlung

- Laserbehandlung Finanzierung/Ratenzahlung

Selbstverständlich kann jede andere Behandlung in unserer Praxis-Klinik finanziert werden.

Wir bieten Ihnen die Möglichkeit, das vereinbarte Behandlungshonorar für Ihre Wunschbehandlung in bequemen und für Sie finanziell möglichen Monatsraten, zu bezahlen.

Gerne übernehmen wir für Sie die komplette Abwicklung. Im Beratungsgespräch legen wir das Behandlungshonorar fest. Wir stellen für Sie den Finanzierungsantrag, der dann von unserem Partner medipay diskret, unbürokratisch und schnell geprüft wird. Sobald die Prüfung abgeschlossen ist und positiv ausfällt, steht Ihrer Wunschbehandlung nichts mehr im Wege..."

\section{Hinweis der Autoren:}

In Gesprächen mit Juristen wurde deutlich, dass die Verbindung von Arzt als medizinischer Leistungserbringer und gleichzeitiger Vermittler einer Finanzdienstleistung rechtlich problematisch sein kann. 


\section{Dermatologie - Medizinisch-kosmetische Leistungen}

\section{Was bietet die Fachgruppe als IGeL-Leistungen an?}

Neben den Dermatologen, den plastischen Chirurgen sind es teilweise die Gynäkologen, die das Internet sehr intensiv für Patienten-Information und Werbung zu IGEL-Leistungen nutzen. Dabei finden sich große Kliniken neben zahlreichen niedergelassenen Praxen.

Abgedeckt wird in den Angeboten zu med. kosmetischen - in der Regel operativen -Leistungen auf den Internetseiten dermatologischer, HNO-ärztlicher, kieferchirurgischer und chirurgischer Praxen und Kliniken ein breites Spektrum aus dem Bereich der ästhetischkosmetischen Dermatologie.

Unter www.derma.med.uni-erlangen.de/e1662/e1998/index ger.html bietet die Hautklinik des Universitätsklinikums Erlangen an:

\section{IGeL-Leistungen}

... „Für den Menschen ist die menschliche Gestalt das Schönste, ... (Chamisso) ... Doch nicht immer bietet eine herkömmliche Therapie auch ein individuell befriedigendes kosmetisches Ergebnis oder man möchte selbst im „besten Alter“ der inneren Jugend optischen Ausdruck verleihen.

Unser Ziel ist es, mittels professioneller ästhetischer Kosmetik und modernster Lasertherapie Ihr Wohlbefinden zusätzlich zu steigern und Ihr Hautbild zu verbessern.

Wir bieten Ihnen folgende Individuelle Gesundheits Leistungen (IGeL) an:

\begin{tabular}{|c|c|}
\hline Anti-Aging-Beratung & $\begin{array}{l}\text { 1. Hauttypbestimmung } \\
\text { 2. individuelle Beratung über Möglichkeiten der Faltenglättung, } \\
\text { sonstiger störender Alterserscheinungen (z. B. Pigmentstörun- } \\
\text { gen, Behaarung) } \\
\text { 3. Prävention }\end{array}$ \\
\hline Hauttyp-Beratung & $\begin{array}{l}\text { 1. Hauttypbestimmung } \\
\text { 2. individuelle Beratung zur Hautpflege } \\
\text { 3. Beratung bei Couperose }\end{array}$ \\
\hline Fruchtsäurepeeling & $\begin{array}{l}\text { 1. Aknenarben (leichte Formen) } \\
\text { 2. Therapie lichtgeschädigter Haut (sog. Altersflecken) } \\
\text { 3. Verfeinerung des Hautbildes } \\
\text { 4. leichte Mimikfalten } \\
\text { 5. großporige Haut }\end{array}$ \\
\hline TCA-Peeling & $\begin{array}{l}\text { 1. tiefe Aknenarben } \\
\text { 2. großporige Haut }\end{array}$ \\
\hline Botox-Injektionen & $\begin{array}{l}\text { 1. Mimikfalten } \\
\text { 2. Hyperhidrose (übermäßiges Schwitzen) }\end{array}$ \\
\hline Hyaluronsäure-Injektionen & $\begin{array}{l}\text { 1. Gewebsaugmentation } \\
\text { 2. Auffüllen von Falten } \\
\text { 3. Auffüllen von eingesunkenen Narben }\end{array}$ \\
\hline Camouflage & $\begin{array}{l}\text { 1. Vitiligo } \\
\text { 2. Pigmentstörungen } \\
\text { 3. Feuermale } \\
\text { 4. Hauterkrankungen mit störendem Hautbild } \\
\text { z. B. Rosazea, Lupus erythematodes, Sarkoidose, etc.) }\end{array}$ \\
\hline Lasertherapie & $\begin{array}{l}\text { 1. Hämangiome } \\
\text { 2. Blutschwämmchen } \\
\text { 3. Teleangiektasien } \\
\text { 4. Besenreiser } \\
\text { 5. Haarentfernung } \\
\text { 6. Altersflecken } \\
\text { 7. etc. }\end{array}$ \\
\hline Laser-Skin-Resurfacing & $\begin{array}{l}\text { 1. therapieresistente Aknenarben } \\
\text { 2. tiefe therapieresistente Mimikfalten }\end{array}$ \\
\hline
\end{tabular}




\begin{tabular}{|l|l|}
\hline Medizinisch-kosmetische & $\begin{array}{l}\text { 1. manuelle Aknetherapie } \\
\text { Behandlungen }\end{array}$ \\
$\begin{array}{l}\text { 2. Milien } \\
\text { 3. Hautreinigung } \\
\text { 4. Spezielle Gesichtsmassage z. B. bei Rosazea }\end{array}$ \\
\hline $\begin{array}{l}\text { Operative Therapie } \\
\text { gutartiger Hauttumore }\end{array}$ & $\begin{array}{l}\text { 1. Muttermale } \\
\text { 2. Alterswarzen } \\
\text { 3. Fibrome (,wildes Fleisch“) }\end{array}$ \\
\hline Schönheitschirurgie & $\begin{array}{l}\text { 1. Augenlidstraffung } \\
\text { 2. Mundwinkel-Lifting } \\
\text { 3. wulstige Narben }\end{array}$ \\
\hline
\end{tabular}

Die niedergelassenen Dermatologen bieten noch weit mehr IGeL-Leistungen an z. B.:

- Akupunktur

- Allergietestung auf Patientenwunsch

- Behandlung von übermäßigem Schwitzen (Hyperhidrose) mit Botulinumtoxin

- Androgenetische Alopezie/Glatzenbehandlung bei Männer

- Balneotherapie zur Behandlung von Hautkrankheiten

- Beratungen und Untersuchungen für Berufe mit Hautgefährdung z. B. chem. Industrie etc.

- Eigenblutbehandlung

- Digitale Muttermal-Dokumentation und Analyse (Fotofinder)

- Haarausfall bei der Frau

- Haaranalyse mit Trichoscan

- Hautabschleifen

- Hautveränderungen - Verlaufkontrollen

- Kosmetische Operationen

- Kosmetika-Verträglichkeit - Testung

- Laserbehandlung von Nagelpilz

- Naturheilverfahren

- Permanent Make up

- Phlebologie

- Photodynamische Therapie bei Hautkrebs und Hautkrebsvorstufen

- Piercing

- Entfernung von Tätowierungen

- UV-Bestrahlung zur Prophylaxe einer Sonnenexposition

Aknebehandlung aus kosmetischen Gründen

\begin{tabular}{|c|c|c|c|}
\hline GOÄ Nr. & Kurzlegende & 1 fach $€$ & $\begin{array}{r}* 1,8 / \\
2,3 f a c h €\end{array}$ \\
\hline 3 & $\begin{array}{l}\text { Eingehende Beratung (mind. } 10 \text { Min.) - nicht neben Sonderleis- } \\
\text { tungen }\end{array}$ & 8,74 & 20,11 \\
\hline 5 & Symptombezogene Untersuchung & 4,66 & 10,73 \\
\hline 7 & Untersuchung Haut & 9,33 & 21,45 \\
\hline $\begin{array}{l}520^{*} \\
\text { analog }\end{array}$ & $\begin{array}{l}\text { Aknemassage - analog GOÄ Nr. } 520 \text { (alternativ Nr. 523) - } \\
\text { entspr. GOÄ § } 6(2)^{1)} \\
\text { GOÄ Kurztext Nr. 520: Teilmassage... }\end{array}$ & 2,62 & 4,72 \\
\hline $530^{*}$ & Aknepackung, je Sitzung & 2,04 & 3,67 \\
\hline $758^{*}$ & Sticheln, Öffnen; Ausquetschen Aknepusteln, je Sitzung & 4,37 & 10,05 \\
\hline 209 & Großflächiges Auftragen von Externa & 8,74 & 20,11 \\
\hline
\end{tabular}

1) Bei analogen Leistungen muss - mind. in Kurzform - auch die Original-Leistungslegende angegeben werden. 
Allergie-Testung auf Patientenwunsch

\begin{tabular}{|c|c|c|c|}
\hline GOÄ Nr. & Kurzlegende & 1fach $€$ & $\begin{array}{r}{ }^{*} 1,8 / \\
2,3 \mathrm{fach} €\end{array}$ \\
\hline 1 & Beratung, auch tel. & 4,66 & 10,72 \\
\hline 3 & Eingehende Beratung, auch tel. & 8,74 & 20,11 \\
\hline 5 & Symptombezogene Untersuchung & 4,66 & 10,73 \\
\hline 7 & $\begin{array}{l}\text { Untersuchung mind. eines Organssystems: Bauchorgane - } \\
\text { Brustorgane - weibl. Genitaltrakt - Haut - Stütz- u. Bewe- } \\
\text { gungsorgane }\end{array}$ & 9,33 & 21,45 \\
\hline $250^{*}$ & Blutentnahme aus Vene & 2,33 & 4,20 \\
\hline 385 & Pricktest, je Test (1.-20. Test je Behandlungsfall) & 2,62 & 6,03 \\
\hline 386 & Pricktest, je Test (21.-40. Test je Behandlungsfall) & 1,75 & 4,02 \\
\hline GOÄ Nr. & Kurzlegende & 1 fach $€$ & *1,15fach $€$ \\
\hline $3572^{*}$ & Immunglobulin E (IgE) & 14,57 & 16,76 \\
\hline $3891^{*}$ & Einzelallergentest $\mathrm{z}$. B. RAST & 14,57 & 16,76 \\
\hline
\end{tabular}

Androgenetischen Alopezie bei Männern

Behandlung der androgenetischen Alopezie bei Männern (Glatzenbehandlung) z. B. mit Nicht-GKVArzneimitteln

\begin{tabular}{|c|c|c|c|}
\hline GOÄ Nr. & Kurzlegende & 1 fach $€$ & $\begin{array}{r}{ }^{*} 1,8 / \\
2,3 f a c h €\end{array}$ \\
\hline 3 & $\begin{array}{l}\text { Eingehende Beratung (mind. } 10 \text { Min.) - nicht neben Sonderleis- } \\
\text { tungen }\end{array}$ & 8,74 & 20,11 \\
\hline 5 & Symptombezogene Untersuchung & 4,66 & 10,73 \\
\hline 7 & Untersuchung Haut - Dokumentation von ev. Nebenwirkungen & 9,33 & 21,45 \\
\hline 8 & Ganzkörperstatus - Dokumentation von ev. Nebenwirkungen & 15,16 & 34,86 \\
\hline $\begin{array}{l}612^{*} \\
\text { analog }\end{array}$ & $\begin{array}{l}\text { Digitale Analyse - epiluminiszenz unterstützte Trichogramm - } \\
\text { Analyse - analog GOÄ Nr. } 612^{*}-\text { entspr. GOÄ § } 6(2)^{1)} \\
\text { Empfehlung der BÄK - GOÄ Kurztext Nr. } 612: \text { Ganzkörper-plethysmographi- } \\
\text { sche Bestimmung der absoluten und relativen Sekundenkapazität und des } \\
\text { Atemwegwiderstandes... }\end{array}$ & 44,12 & 79,42 \\
\hline $4860^{*}$ & $\begin{array}{l}\text { Trichogramm } \\
\text { Ggf. Kontrolluntersuchungen nach einigen Monaten - bei Tri- } \\
\text { chophotogramm höherer Steigerungssatz + Sachkosten der } \\
\text { Photodokumentation nach } \$ 10 \text { berechenbar }\end{array}$ & 9,33 & $16,79^{\star *}$ \\
\hline
\end{tabular}

1) Bei analogen Leistungen muss - mind. in Kurzform - auch die Original-Leistungslegende angegeben werden.

${ }^{* \star}=1,15$ facher Satz (Laborleistung)

Weitere Laboruntersuchungen ggf.:

- Biotin

- FAl (Freier Androgen Index)

- Schilddrüsenhormone

- SHBG (Sexualhormonbindendes Globulin)

- Testosteron

Balneotherapie zur Behandlung von Hautkrankheiten

\begin{tabular}{|l|l|r|r|}
\hline GOÄ Nr. & Kurzlegende & $\mathbf{1 f a c h} €$ & $\begin{array}{r}{ }^{* 1,8 /} \\
\mathbf{2 , 3 f a c h} €\end{array}$ \\
\hline $\mathbf{3}$ & $\begin{array}{l}\text { Eingehende Beratung (mind. 10 Min.) - nicht neben Sonderleis- } \\
\text { tungen }\end{array}$ & 8,74 & $\mathbf{2 0 , 1 1}$ \\
\hline $\mathbf{5}$ & Symptombezogene Untersuchung & 4,66 & $\mathbf{1 0 , 7 3}$ \\
\hline
\end{tabular}




\begin{tabular}{|c|c|c|c|}
\hline GOÄ Nr. & Kurzlegende & 1 fach $€$ & $\begin{array}{r}{ }^{*} 1,8 / \\
2,3 \text { fach } €\end{array}$ \\
\hline 7 & Untersuchung Haut & 9,33 & 21,45 \\
\hline \multicolumn{4}{|c|}{ Synchrone Balneotherapie (z. B. „Kieler-Modell“) } \\
\hline $566^{*}$ & $\begin{array}{l}\text { Synchrone Balneotherapie, je Behandlung, analoger Ansatz der } \\
\text { Nr. } 566 \text { - GOÄ § } 6 \text { (2) } \\
\text { Empfehlung der BÄK - GOÄ Kurztext Nr. 566: Phototherapie eines Neugeborenen }\end{array}$ & 29,14 & 52,46 \\
\hline \multicolumn{4}{|c|}{ Asynchrone Balneotherapie (z. B. „Tomesa-Konzept“) } \\
\hline $532 a^{*}$ & Leitung eines ansteigenden Vollbades & 4,43 & 7,97 \\
\hline $565 a^{*}$ & Photochemotherapie, je Sitzung & 6,99 & 12,69 \\
\hline
\end{tabular}

Sachkosten nach $\S 10$ berechnen.

Beratungen und Erörterungen zu medizinisch-kosmetischen Problemen

Beratungen, die abhängig von der Beratungsintensität nach den GOÄ Nrn. 1 oder 3 oder als Erörterung nach Nr. 34 abgerechnet werden können:

- Allgemeine medizinisch kosmetische Beratung

- Sonnenlicht- und Hauttypberatung

- Beratung vor kosmetischen Behandlungen oder kosmetisch-operativen Eingriffen

- Beratung vor einer gewünschten Arzneimittel-Behandlung z. B. „Life-Style“ gegen androgenetischen Haarausfall

- Second opinion - Zweitmeinung auf Patientenwunsch

\begin{tabular}{|l|l|r|r|}
\hline GOÄ Nr. & Kurzlegende & $\mathbf{1 f a c h} €$ & $\mathbf{2 , 3 f a c h} €$ \\
\hline $\mathbf{3}$ & $\begin{array}{l}\text { Eingehende Beratung (mind. 10 Min.) - nicht neben Sonderleis- } \\
\text { tungen }\end{array}$ & 8,74 & $\mathbf{2 0 , 1 1}$ \\
\hline $\mathbf{1}$ & Beratung & 4,66 & $\mathbf{1 0 , 7 3}$ \\
\hline $\mathbf{3 4}$ & Erörterung, mind. 20 Min. z. B. & 17,49 & $\mathbf{4 0 , 2 2}$ \\
\hline
\end{tabular}

Besenreiservarizen - Verödung

Die Abrechnung ist abhängig von der gewählten Methode.

\begin{tabular}{|c|c|c|c|}
\hline GOÄ Nr. & Kurzlegende & 1 fach $€$ & $\begin{array}{r}{ }^{*} 1,8 / \\
2,3 f a c h €\end{array}$ \\
\hline 1 & Beratung, auch tel. & 4,66 & 10,72 \\
\hline 3 & Eingehende Beratung, auch tel. & 8,74 & 20,11 \\
\hline 5 & Symptombezogene Untersuchung & 4,66 & 10,73 \\
\hline 204 & $\begin{array}{l}\text { stabilisierender Verband einer Extremität über mind. zwei große } \\
\text { Gelenke; Kompressionsverband }\end{array}$ & 5,54 & 12,74 \\
\hline 634 & Lichtreflex-Rheographie & 6,99 & 16,09 \\
\hline $\begin{array}{l}706 \\
\text { analog }\end{array}$ & $\begin{array}{l}\text { Verödung von Besenreiservarizen mit gepulstem Licht, je Sitzung } \\
\text { - analog Nr. } 706 \text { Licht- oder Laserkoagulation(en) - entspr. GOA } \\
\S 6 \text { (2) }\end{array}$ & 34,97 & 80,44 \\
\hline 764 & Verödung (Sklerosierung) von Krampfadern, je Sitzung & 11,07 & 25,47 \\
\hline $\begin{array}{l}2440 \\
\text { analog }\end{array}$ & $\begin{array}{l}\text { Laserbehandlung von Besenreiservarizen, einschl. Laser-Epila- } \\
\text { tion, mit einer Ausdehnung bis zu } 7 \mathrm{~cm}^{2} \text { Körperoberfläche, bis zu } \\
\text { dreimal im Behandlungsfall, im Falle der Behandlung von Besen- } \\
\text { reiservarizen mit einer Laser-Impulsrate von bis zu } 50 \text { Impulsen } \\
\text { pro Sitzung (analog Nr. } 2440 \text { GOÄ) - n. Beschlüssen des Aus- } \\
\text { schusses „Gebührenordnung“ der BÄK - analog GOÄ Nr. } 2440 \text { - } \\
\text { entspr. GÖ̈ § } 6(2)^{1)}\end{array}$ & 6,63 & 107,25 \\
\hline
\end{tabular}

1) Bei analogen Leistungen muss - mind. in Kurzform - auch die Original-Leistungslegende angegeben werden. 
Therapie von Gefäßveränderungen auch mittels Laser

\begin{tabular}{|c|c|c|c|}
\hline GOÄ Nr. & Kurzlegende & 1 fach $€$ & 2,3 fach $€$ \\
\hline $\begin{array}{l}764 \\
\text { analog }\end{array}$ & $\begin{array}{l}\text { Spider Naevi u.senile Hämangiome, je Läsion - analog GOÄ } \\
\text { Nr. } 764 \text { - entspr. GOÄ § } 6(2)^{1)}\end{array}$ & 11.07 & 25,47 \\
\hline $\begin{array}{l}2440 \\
\text { analog }\end{array}$ & $\begin{array}{l}\text { Besenreiser pro Bein je nach Ausdehnung, je Sitzung - } \\
\text { analog GOÄ Nr. } 2440 \text { - entspr. GOÄ § } 6(2)^{1)} \\
\text { GOÄ Kurztext Nr. 2440: Operative Entfernung eines Naevus flammeus... }\end{array}$ & 46,63 & 107,25 \\
\hline $\begin{array}{l}2440 \\
\text { analog }\end{array}$ & $\begin{array}{l}\text { Rubeosis teleangiektatika, je Sitzung - analog GOÄ Nr. } 2440 \text { - } \\
\text { entspr. GOÄ § } 6(2)^{1)} \\
\text { GOÄ Kurztext Nr. 2440: Operative Entfernung eines Naevus flammeus... }\end{array}$ & 46,63 & 107,25 \\
\hline 444 & **Zuschlag zu Nr. 2440 bei ambulanter Op & 75,77 & -- \\
\hline
\end{tabular}

1) Bei analogen Leistungen muss - mind. in Kurzform - auch die Original-Leistungslegende angegeben werden.

Laserbehandlung in der Dermatologie sind nach den GOÄ Nrn. 2440 oder 2885 oder 2886 abzurechnen!

\section{Eigenblutbehandlung}

\begin{tabular}{|l|l|r|r|}
\hline GOÄ Nr. & Kurzlegende & $\mathbf{1 f a c h} €$ & $\mathbf{2 , 3 f a c h} €$ \\
\hline $\mathbf{3}$ & $\begin{array}{l}\text { Eingehende Beratung (mind. 10 Min.) - nicht neben Sonderleis- } \\
\text { tungen }\end{array}$ & 8,74 & $\mathbf{2 0 , 1 1}$ \\
\hline $\mathbf{5}$ & Symptombezogene Untersuchung Haut & 4,66 & $\mathbf{1 0 , 7 3}$ \\
\hline $\mathbf{2 8 4}$ & Eigenblutbehandlung einschl. Blutentnahme & 5,25 & $\mathbf{1 2 , 0 7}$ \\
\hline
\end{tabular}

Epilation störender Behaarung außer bei krankhaftem und entstellendem Haarwuchs an Händen und im Gesicht

\begin{tabular}{|l|l|r|r|}
\hline GOÄ Nr. & Kurzlegende & $\mathbf{1 f a c h} \boldsymbol{\text { }}$ & $\mathbf{2 , 3 f a c h} \mathbf{~}$ \\
\hline $\mathbf{3}$ & $\begin{array}{l}\text { Eingehende Beratung (mind. 10 Min.) - nicht neben Sonderleis- } \\
\text { tungen }\end{array}$ & 8,74 & $\mathbf{2 0 , 1 1}$ \\
\hline $\mathbf{5}$ & Symptombezogene Untersuchung Haut & 4,66 & $\mathbf{1 0 , 7 3}$ \\
\hline $\mathbf{7 4 2}$ & $\begin{array}{l}\text { Epilation von Haaren außer bei krankhaftem und entstellendem } \\
\text { Haarwuchs an Händen und im Gesicht }\end{array}$ & 9,62 & $\mathbf{2 2 , 1 2}$ \\
\hline $\begin{array}{l}\mathbf{7 0 6} \\
\mathbf{a n a l o g}\end{array}$ & $\begin{array}{l}\text { Kleinflächige Epilation von Haaren mit gepulstem Licht, je Sit- } \\
\text { zung - analog GOÄ Nr. 706 - entspr. GOÄ § 6 (2) } \\
\text { GOÄ Kurztext Nr. 706: Licht- oder Laserkoagulation(en)... }\end{array}$ & 34,97 & $\mathbf{8 0 , 4 4}$ \\
\hline $\mathbf{1 3 2 3}$ & Elektrolytische Epilation von Wimpernhaaren, je Sitzung & 3,91 & $\mathbf{8 , 9 8}$ \\
\hline
\end{tabular}

1) Bei analogen Leistungen muss - mind. in Kurzform - auch die Original-Leistungslegende angegeben werden.

Falten- oder Narbenunterspritzung

Collagen o. körpereigenem Fettgewebe Implantationstechniken (Kollagen/Zyderm, Zyplast u. a.)

\begin{tabular}{|l|l|r|r|}
\hline GOÄ Nr. & Kurzlegende & $\mathbf{1 f a c h} €$ & $\mathbf{2 , 3 f a c h ~} \mathbf{}$ \\
\hline $\mathbf{3}$ & $\begin{array}{l}\text { Eingehende Beratung (mind. 10 Min.) - nicht neben Sonderleis- } \\
\text { tungen }\end{array}$ & 8,74 & $\mathbf{2 0 , 1 1}$ \\
\hline $\mathbf{3 4}$ & Erörterung & 17,49 & $\mathbf{4 0 , 2 3}$ \\
\hline $\mathbf{3 1}$ & $\begin{array}{l}\text { Anti-Aging-Gespräch - analog - GOÄ § 6 (2) } \\
\text { GOÄ Kurztext Nr. 31: Homöopathische Folgeanamnese }\end{array}$ & 26,23 & $\mathbf{6 0 , 3 3}$ \\
\hline $\mathbf{3 9 0}$ & Testspritze & 3,50 & $\mathbf{8 , 0 4}$ \\
\hline $\mathbf{2 4 4 2}$ & Implantation alloplastischen Materials zur Weichteilunterfütterung & 52,46 & $\mathbf{1 2 0 , 6 6}$ \\
\hline
\end{tabular}

1) Bei analogen Leistungen muss - mind. in Kurzform - auch die Original-Leistungslegende angegeben werden.

+ Materialkosten gemäß GOÄ § 10 abrechnen 


\section{E. Individuelle Gesundheitsleistungen von A - Z}

Haarausfall bei der Frau

Zur Abklärung des Haarausfalles bei der Frau finden neben den oben bei Androgenetischen Alopezie bei Männern genannten Leistungen folgende Laboruntersuchungen Anwendung:

- Biotin

- DHEAS

- FAl (Freier Androgen Index)

- Ferritin

- $\mathrm{FSH}$

- LH

- Östradiol

- Schilddrüsenhormone

- SHBG (Sexualhormonbindendes Globulin)

- Testosteron

Hautabschleifen

\begin{tabular}{|l|l|r|r|}
\hline GOÄ Nr. & Kurzlegende & $\mathbf{1 f a c h} €$ & $\begin{array}{r}\text { *1,8/ } \\
\mathbf{2 , 3 f a c h ~} €\end{array}$ \\
\hline $\begin{array}{l}\mathbf{7 5 5} \\
\text { analog }\end{array}$ & $\begin{array}{l}\text { Microabrasion z. B. mittels Skinabrader, je Sitz. - analog GOÄ } \\
\text { Nr. 755 - entspr. GOÄ § 6 (2) }\end{array}$ & 13,99 & $\mathbf{3 2 , 1 8}$ \\
\hline $\begin{array}{l}\mathbf{2 4 4 1} \\
\text { analog }\end{array}$ & $\begin{array}{l}\text { niedertourige Dermabrasio - analog GOÄ Nr. 2441 - entspr. } \\
\text { GOÄ § 6 (2) }\end{array}$ & 23,31 & $\mathbf{5 3 , 6 2}$ \\
\hline $\mathbf{4 4 2}$ & Zuschlag zu Nr. 2441 bei ambulanter Op & $\mathbf{2 3 , 3 1}$ & - \\
\hline
\end{tabular}

1) Bei analogen Leistungen muss - mind. in Kurzform - auch die Original-Leistungslegende angegeben werden.

Bei hochtourige Dermabrasio, je Sitzung - mehrfacher Ansatz

\section{Haut-Check}

\begin{tabular}{|l|l|r|r|}
\hline GOÄ Nr. & Kurzlegende & $\mathbf{1}$ fach $€$ & $\begin{array}{r}{ }^{*} \mathbf{1 , 8 /} \\
\mathbf{2 , 3 f a c h ~} \mathbf{1}\end{array}$ \\
\hline $\mathbf{1}$ & Beratung, auch tel. & 4,66 & $\mathbf{1 0 , 7 2}$ \\
\hline $\mathbf{3}$ & Eingehende Beratung, auch tel. & 8,74 & $\mathbf{2 0 , 1 1}$ \\
\hline $\mathbf{5}$ & Symptombezogene Untersuchung & 4,66 & $\mathbf{1 0 , 7 3}$ \\
\hline $\mathbf{7}$ & $\begin{array}{l}\text { Untersuchung mind. eines Organssystems: Bauchorgane - } \\
\text { Brustorgane - weibl. Genitaltrakt - Haut - Stütz- u. Bewegungs- } \\
\text { organe }\end{array}$ & 9,33 & $\mathbf{2 1 , 4 5}$ \\
\hline $\mathbf{7 5 0}$ & Auflichtmikroskopie, je Sitzung & 6,99 & $\mathbf{1 6 , 0 9}$ \\
\hline $\begin{array}{l}\mathbf{6 1 2} \\
\text { analog }\end{array}$ & $\begin{array}{l}\text { Videosystem gestützte Untersuchung und Bilddokumentation } \\
\text { von Hautmalen - analog GOÄ Nr. 612 - entspr. GOÄ § 6 (2) })^{1)}\end{array}$ & 44,12 & $\mathbf{7 9 , 4 2}$ \\
\hline
\end{tabular}

1) Bei analogen Leistungen muss - mind. in Kurzform - auch die Original-Leistungslegende angegeben werden.

Haut-Peeling

Die Abrechnung ist abhängig von der gewählten Methode.

\begin{tabular}{|c|c|c|c|}
\hline GOÄ Nr. & Kurzlegende & 1 fach $€$ & $\begin{array}{r}{ }^{*} 1,8 / \\
2,3 f a c h €\end{array}$ \\
\hline 1 & Beratung, auch tel. & 4,66 & 10,72 \\
\hline 3 & Eingehende Beratung, auch tel. & 8,74 & 20,11 \\
\hline 5 & Symptombezogene Untersuchung & 4,66 & 10,73 \\
\hline 209 & $\begin{array}{l}\text { Großflächiges Auftragen von Externa zur Behandlung von } \\
\text { Hautkrankheiten mind. einer Körperregion je Sitzung }\end{array}$ & 8,74 & 20,11 \\
\hline 743 & $\begin{array}{l}\text { Schleifen und Schmirgeln und/oder Fräsen von Bezirken der } \\
\text { Haut, je Sitzung }\end{array}$ & 4,37 & 10,05 \\
\hline 755 & $\begin{array}{l}\text { Hochtouriges Schleifen von Bezirken der Haut bei schweren } \\
\text { Entstellungen, je Sitzung }\end{array}$ & 13,99 & 32,17 \\
\hline
\end{tabular}


Haut-Typberatung

\begin{tabular}{|l|l|r|r|}
\hline GOÄ Nr. & Kurzlegende & $\mathbf{1 f a c h} €$ & $\begin{array}{r}{ }^{* 1,8 /} \\
\mathbf{2 , 3 f a c h} €\end{array}$ \\
\hline $\mathbf{1}$ & Beratung, auch tel. & 4,66 & $\mathbf{1 0 , 7 2}$ \\
\hline $\mathbf{3}$ & Eingehende Beratung, auch tel. & 8,74 & $\mathbf{2 0 , 1 1}$ \\
\hline $\mathbf{5}$ & Symptombezogene Untersuchung & 4,66 & $\mathbf{1 0 , 7 3}$ \\
\hline $\mathbf{7}$ & $\begin{array}{l}\text { Untersuchung mind. eines Organssystems: Bauchorgane - Brust- } \\
\text { organe - weibl. Genitaltrakt - Haut - Stütz- u. Bewegungsorgane }\end{array}$ & 9,33 & $\mathbf{2 1 , 4 5}$ \\
\hline $\mathbf{7 6 0}$ & Alkaliresistenzbestimmung & 7,05 & $\mathbf{1 2 , 6 9}$ \\
\hline
\end{tabular}

Hautveränderungen - Verlaufskontrolle von Muttermalen

\begin{tabular}{|c|c|c|c|}
\hline GOÄ Nr. & Kurzlegende & 1 fach $€$ & $\begin{array}{r}{ }^{*} 1,8 / \\
2,3 f a c h €\end{array}$ \\
\hline 3 & $\begin{array}{l}\text { Eingehende Beratung (mind. } 10 \text { Min.) - nicht neben Sonderleis- } \\
\text { tungen }\end{array}$ & 8,74 & 20,11 \\
\hline 7 & Untersuchung Haut & 9,33 & 21,45 \\
\hline 750 & Auflichtmikroskopie (Dermatoskopie), je Sitzung & 6,99 & 16,09 \\
\hline $\begin{array}{l}612^{\star} \\
\text { analog }\end{array}$ & $\begin{array}{l}\text { Video-Dermatoskopie - analog GOÄ Nr. } 612 \text { - entspr. GOÄ } \S 6(2)^{1)} \\
\text { Empfehlung der BÄK - GOÄ Kurztext Nr. 612: Ganzkörperplethysmographische } \\
\text { Bestimmung der absoluten und relativen Sekundenkapazität }\end{array}$ & 44,12 & 79,42 \\
\hline
\end{tabular}

1) Bei analogen Leistungen muss - mind. in Kurzform - auch die Original-Leistungslegende angegeben werden.

Verschiedene kosmetische Operationen

Natürlich gehören zu den einzelnen OPs noch weitere Leistungen (z. B. Anaesthesie, etc.)

\begin{tabular}{|c|c|c|c|}
\hline GOÄ Nr. & Kurzlegende & 1 fach $€$ & 2,3 fach $€$ \\
\hline 3 & $\begin{array}{l}\text { Eingehende Beratung (mind. } 10 \text { Min.) - nicht neben Sonderleis- } \\
\text { tungen }\end{array}$ & 8,74 & 20,11 \\
\hline 5 & Symptombezogene Untersuchung & 4,66 & 10,73 \\
\hline 1447 & Nasenkorrektur & 96,76 & 222,54 \\
\hline $\begin{array}{l}1304 \\
\text { analog }\end{array}$ & $\begin{array}{l}\text { Lidkorrektur - analog GOÄ Nr. } 1304 \text { - entspr. GOÄ } \S 6(2)^{1)} \\
\text { GOÄ Kurztext Nr. 1304:Plastische Korrektur des Ektropiums oder Entropiums, der } \\
\text { Trichiasis oder Distichiasis }\end{array}$ & 53,86 & 123,87 \\
\hline 1310 & Augenlidplastik mittels freien Hauttransplantates & 86,27 & 198,41 \\
\hline 1311 & Augenlidplastik mittels Hautlappenverschiebung & 64,70 & 148,81 \\
\hline $\begin{array}{l}2454 \\
\text { analog }\end{array}$ & $\begin{array}{l}\text { Fettentfernung - analog GOÄ Nr. } 2454 \text { - entspr. GOÄ § } 6(2)^{1)} \\
\text { GOÄ Kurztext Nr. 2454: Operative Entfernung von überstehendem Fettgewebe an } \\
\text { einer Extremität }\end{array}$ & 53,86 & 123,87 \\
\hline $\begin{array}{l}2452 \\
\text { analog }\end{array}$ & $\begin{array}{l}\text { Fettschürzenentfernung (z. B. Bauchdecke) - analog GOÄ } \\
\text { Nr. } 2452 \text { - entspr. GOÄ § } 6(2)^{1)} \\
\text { GOÄ Kurztext Nr. 2452: Exstirpation einer Fettschürze }\end{array}$ & 81,60 & 187,69 \\
\hline $\begin{array}{l}2453 \\
\text { analog }\end{array}$ & $\begin{array}{l}\text { Fettentfernung (Extremitäten) - analog GOÄ Nr. } 2453 \text { - entspr. } \\
\text { GOÄ § } 6(2)^{1)} \\
\text { GOÄ Kurztext Nr. 2453: Operation des Lymphödems einer Extremität }\end{array}$ & 116,57 & 268,12 \\
\hline
\end{tabular}

1) Bei analogen Leistungen muss - mind. in Kurzform - auch die Original-Leistungslegende angegeben werden.

Behandlung von Krampfadern z.Zt. ohne Krankheitswert

Die Abrechnung ist abhängig von der gewählten Methode.

\begin{tabular}{|l|l|r|r|}
\hline GOÄ Nr. & Kurzlegende & $\mathbf{1 f a c h} €$ & $\begin{array}{r}{ }^{*} \mathbf{1 , 8 /} \\
\mathbf{2 , 3 f a c h} €\end{array}$ \\
\hline $\mathbf{1}$ & Beratung, auch tel. & 4,66 & $\mathbf{1 0 , 7 2}$ \\
\hline $\mathbf{3}$ & Eingehende Beratung, auch tel. & 8,74 & $\mathbf{2 0 , 1 1}$ \\
\hline
\end{tabular}




\begin{tabular}{|c|c|c|c|}
\hline GOÄ Nr. & Kurzlegende & 1 fach $€$ & $\begin{array}{r}{ }^{*} 1,8 / \\
2,3 f a c h €\end{array}$ \\
\hline 5 & Symptombezogene Untersuchung & 4,66 & 10,73 \\
\hline $639^{*}$ & $\begin{array}{l}\text { photoplethysmographische Registrierung der Blutfüllung und } \\
\text { photoplethysmographische Simultanregistrierung der Füllungs- } \\
\text { schwankungen peripherer Arterien an mind. } 4 \text { peripheren Gefäß- } \\
\text { abschnitten - gleichzeitige Registrierung des Volumenpulsbandes }\end{array}$ & 26,46 & 47,63 \\
\hline $643^{*}$ & $\begin{array}{l}\text { Periphere Arterien- bzw. Venendruck- und/oder Strömungsmes- } \\
\text { sung }\end{array}$ & 6,99 & 12,59 \\
\hline $644^{*}$ & $\begin{array}{l}\text { Untersuchung der Strömungsverhältnisse in Extremitätenarterien } \\
\text { bzw. -venen mit direktionaler Ultraschall-Doppler-Technik - } \\
\text { einschl. graphischer Registrierung - }\end{array}$ & 10,49 & 18,89 \\
\hline 764 & Verödung (Sklerosierung) von Krampfadern, je Sitzung & 11,07 & 25,47 \\
\hline 204 & $\begin{array}{l}\text { stabilisierender Verband einer Extremität über mind. zwei große } \\
\text { Gelenke; Kompressionsverband }\end{array}$ & 5,54 & 12,74 \\
\hline
\end{tabular}

Abrechnung der Sachkosten gemäß § 10 GOÄ

Peeling zur Verbesserung des Hautreliefs/Fruchtsäure-Peeling

\begin{tabular}{|l|l|r|r|}
\hline GOÄ Nr. & Kurzlegende & $\mathbf{1 f a c h} \boldsymbol{€}$ & $\begin{array}{r}{ }^{* 1,8 /} \\
\mathbf{2 , 3 f a c h} €\end{array}$ \\
\hline $\mathbf{3}$ & $\begin{array}{l}\text { Eingehende Beratung (mind. 10 Min.) - nicht neben Sonderleis- } \\
\text { tungen }\end{array}$ & 8,74 & $\mathbf{2 0 , 1 1}$ \\
\hline $\mathbf{5}$ & Symptombezogene Untersuchung & 4,66 & $\mathbf{1 0 , 7 3}$ \\
\hline $\mathbf{2 0 9}$ & Großflächige Hautbehandlung - analoger Ansatz Nr. 209 & 8,74 & $\mathbf{2 0 , 1 1}$ \\
\hline $\mathbf{2 4 4 1}$ & $\begin{array}{l}\text { oberflächlich z. B. Fruchtsäuren, je Sitzung - analog - GOÄ § 6 (2) } \\
\text { GOÄ Kurztext Nr. 2441: Operative Korrektur einer entstellenden Gesichtsnarbe }\end{array}$ & 23,31 & $\mathbf{5 3 , 6 2}$ \\
\hline $\mathbf{4 4 2}$ & Zuschlag zu Nr. 2441 bei ambulanter OP & 23,31 & - \\
\hline $\mathbf{7 4 3}$ & Schleifen und Schmirgeln u./o. Fräsen von Hautbezirken & 4,37 & $\mathbf{1 0 , 0 5}$ \\
\hline $\mathbf{7 5 5}$ & Hochtouriges Schleifen der Haut, je Sitzung & 13,99 & $\mathbf{3 2 , 7 7}$ \\
\hline $\mathbf{7 6 0}$ * & Hauttypbestimmung - analoger Ansatz Nr. 760 & 7,05 & $\mathbf{1 2 , 6 9}$ \\
\hline
\end{tabular}

\section{Abrechnungshinweis:}

- bei mittlere Eindringtiefe z. B. TCA-Peeling, je Sitzung - ggf. mehrfacher Ansatz der oben aufgeführten GOÄ-Nrn. • Materialkosten nach § $10 \mathrm{GOÄ} \mathrm{berechnen}$

Phlebologie

\begin{tabular}{|c|c|c|c|}
\hline GOÄ Nr. & Kurzlegende & 1 fach $€$ & $\begin{array}{r}{ }^{*} 1,8 / \\
2,3 \mathrm{fach} €\end{array}$ \\
\hline 764 & Sklerosierung von kl. Venektasien, je Sitzung & 11,08 & 25,47 \\
\hline 204 & Kompressionsverband & 5,54 & 12,74 \\
\hline $\begin{array}{l}2882 \\
\text { analog }\end{array}$ & $\begin{array}{l}\text { Phlebektomie, je Bein - analog GOÄ Nr. } 2882 \text { - entspr. GOÄ § } 6(2)^{1)} \\
\text { GOÄ Kurztext Nr. 2882: Varizenexhairese mit Unterbrechung der Vv. perforantes, } \\
\text { einseitig }\end{array}$ & 107,83 & 248,01 \\
\hline 445 & Zuschlag zu Nr. 2882 bei ambulanter Op & 128,23 &.- \\
\hline
\end{tabular}

1) Bei analogen Leistungen muss - mind. in Kurzform - auch die Original-Leistungslegende angegeben werden.

Zur Abschätzung des Thrombose-Risikos-Check werden folgende laborchemischer Untersuchungen angeraten:

- Antithrombin-III-Aktivität

- APC-Resistenz (Gerinnungstest)

- Homocystein

- Lupus-Antikoagulans
- Protein-C-Aktivität

- Protein-S-Aktivität 


\section{Piercing}

\begin{tabular}{|c|c|c|c|}
\hline GOÄ Nr. & Kurzlegende & 1fach $€$ & 2,3 fach $€$ \\
\hline 1 & Beratung & 4,66 & 10,72 \\
\hline 5 & Symptombezogene Untersuchung & 4,66 & 10,72 \\
\hline 490 & Infiltrationsanästhesie kleiner Bezirke & 3,56 & 8,18 \\
\hline $\begin{array}{l}2403 \\
\text { analog }\end{array}$ & $\begin{array}{l}\text { Exzision einer kleinen Geschwulst - analog GOÄ Nr. } 2403 \text { - } \\
\text { entspr. GOÄ § } 6(2)^{1)} \\
\text { GOÄ Kurztext Nr. } 2403 \text { : Exzision einer in oder unter der Haut oder Schleimhaut } \\
\text { liegenden kleinen Geschwulst }\end{array}$ & 7,75 & 17,83 \\
\hline $\begin{array}{l}2440 \\
\text { analog }\end{array}$ & $\begin{array}{l}\text { Bei schwierigem Piercing: Operative Entfernung eines Naevis } \\
\text { flammeus, je Sitzung - analog GOÄ Nr. } 2440 \text { - entspr. GOÄ } \S 6(2)^{1)} \\
\text { GOÄ Kurztext Nr. 2440: Operative Entfernung eines Naevus flammeus... }\end{array}$ & 46,63 & 107,25 \\
\hline
\end{tabular}

1) Bei analogen Leistungen muss - mind. in Kurzform - auch die Original-Leistungslegende angegeben werden.

\section{Entfernung von Tätowierungen}

\begin{tabular}{|c|c|c|c|}
\hline GOÄ Nr. & Kurzlegende & 1 fach $€$ & $2,3 f a c h €$ \\
\hline 3 & $\begin{array}{l}\text { Eingehende Beratung (mind. } 10 \text { Min.) - nicht neben Sonderleis- } \\
\text { tungen }\end{array}$ & 8,74 & 20,11 \\
\hline 5 & Symptombezogene Untersuchung & 4,66 & 10,73 \\
\hline 490 & Infiltrationsanästhesie kleiner Bezirke & 3,56 & 8,18 \\
\hline 491 & Infiltrationsanästhesie großer Bezirke & 7,05 & 16,22 \\
\hline 755 & Entfernung von Tätowierungen, Hochtouriges Schleifen der Haut & 2,40 & 13,99 \\
\hline $\begin{array}{l}2440 \\
\text { analog }\end{array}$ & $\begin{array}{l}\text { Operative Entfernung einer Tätowierung mittels Laser, bis zu } \\
7 \mathrm{~cm}^{2} \text {, je Sitzung - analog GOÄ Nr. } 2440 \text { - entspr. GOÄ } \S 6(2)^{1)} \\
\text { Empfehlung der BÄK - GOÄ Kurztext Nr. } 2440 \text { : Operative Entfernung eines } \\
\text { Naevus flammeus... }\end{array}$ & 46,63 & 107,25 \\
\hline 2885 & $\begin{array}{l}\text { Operative Entfernung einer Tätowierung mittels Laser, von } 7 \text { bis } \\
21 \mathrm{~cm}^{2} \text {, je Sitzung - analog GOÄ Nr. } 2885 \text { - entspr. GOÄ } \S 6(2)^{1)} \\
\text { Empfehlung der BÄK - GOÄ Kurztext Nr. 2885: Entfernung einer kleinen Blutader- } \\
\text { geschwulst }\end{array}$ & 64,70 & 148,81 \\
\hline 2886 & $\begin{array}{l}\text { Operative Entfernung einer Tätowierung mittels Laser, mehr als } \\
21 \mathrm{~cm}^{2} \text {, je Sitzung - analog GOÄ Nr. } 2886 \text { - entspr. GOÄ } \S 6(2)^{1)} \\
\text { Empfehlung der BÄK - GOÄ Kurztext Nr. 2886: Entfernung einer großen Blutader- } \\
\text { geschwulst }\end{array}$ & 161,46 & 371,35 \\
\hline
\end{tabular}

1) Bei analogen Leistungen muss - mind. in Kurzform - auch die Original-Leistungslegende angegeben werden.

Tests zur Prüfung der Verträglichkeit von Kosmetika

\begin{tabular}{|l|l|r|r|}
\hline GOÄ Nr. & Kurzlegende & $\mathbf{1 f a c h} €$ & $\begin{array}{r}\mathbf{*}, \mathbf{8} / \\
\mathbf{2 , 3 f a c h} €\end{array}$ \\
\hline $\mathbf{3}$ & $\begin{array}{l}\text { Eingehende Beratung (mind. 10 Min.) - nicht neben Sonderleis- } \\
\text { tungen }\end{array}$ & 8,74 & $\mathbf{2 0 , 1 1}$ \\
\hline $\mathbf{5}$ & Symptombezogene Untersuchung & 4,66 & $\mathbf{1 0 , 7 3}$ \\
\hline $\mathbf{3 8 0}$ & $\begin{array}{l}\text { Tests zur Prüfung der Verträglichkeit von Kosmetika, je Test, } \\
\text { 1.-30. Test }\end{array}$ & 1,75 & $\mathbf{4 , 0 2}$ \\
\hline $\mathbf{3 8 1}$ & $\begin{array}{l}\text { Tests zur Prüfung der Verträglichkeit von Kosmetika, je Test, } \\
\text { 31.-50. Test }\end{array}$ & 1,17 & $\mathbf{2 , 6 8}$ \\
\hline $\mathbf{3 8 8}$ & $\begin{array}{l}\text { Reib-, Scratch- oder Skarifikationstest je Test (bis zu 10 je } \\
\text { Behandlungsfall) }\end{array}$ & 2,04 & $\mathbf{4 , 6 9}$ \\
\hline
\end{tabular}

UV-Bestrahlung zur Prophylaxe einer Sonnenexposition

\begin{tabular}{|l|l|r|r|}
\hline GOÄ Nr. & Kurzlegende & $\mathbf{1 f a c h} €$ & $\begin{array}{r}\text { *1,8/ } \\
\mathbf{2 , 3 f a c h} €\end{array}$ \\
\hline $\mathbf{1}$ & Beratung, auch tel. & 4,66 & $\mathbf{1 0 , 7 2}$ \\
\hline
\end{tabular}


E. Individuelle Gesundheitsleistungen von A - Z

\begin{tabular}{|l|l|r|r|}
\hline GOÄ Nr. & Kurzlegende & $\mathbf{1 f a c h} €$ & $\begin{array}{r}{ }^{* 1,8 /} \\
\mathbf{2 , 3 f a c h} €\end{array}$ \\
\hline $\mathbf{3}$ & Eingehende Beratung, auch tel. & 8,74 & $\mathbf{2 0 , 1 1}$ \\
\hline $\mathbf{5}$ & Symptombezogene Untersuchung & 4,66 & $\mathbf{1 0 , 7 3}$ \\
\hline $\mathbf{5 6 0 *}$ & Behandlung mit Ultraviolettlicht in einer Sitzung & 1,81 & $\mathbf{3 , 2 5}$ \\
\hline $\mathbf{5 6 1 *}$ & $\begin{array}{l}\text { Reizbehandlung eines umschriebenen Hautbezirkes mit Ultravio- } \\
\text { lettlicht }\end{array}$ & 1,81 & $\mathbf{3 , 2 5}$ \\
\hline $\mathbf{5 6 2 *}$ & $\begin{array}{l}\text { Reizbehandlung mehrerer umschriebener Hautbezirke mit Ultra- } \\
\text { violettlicht in einer Sitzung }\end{array}$ & 2,68 & $\mathbf{4 , 8 3}$ \\
\hline
\end{tabular}

Der Berufsverband der Dermatologen empfahl schon vor Jahren seinen Mitgliedern die folgende Einverständniserklärung für die Patienten.

\section{Sehr verehrte Patientin, sehr geehrter Patient,}

Krankenkassen dürfen nur die Kosten der Behandlung einer Krankheit erstatten. Bestimmte Leistungen aus dem Bereich der Kosmetik und ästhetischen Medizin können daher nicht über Krankenkassen abgerechnet werden. Falls Sie eine solche Behandlung wünschen, werde ich das Honorar auf der Grundlage der Gebührenordnung für Ärzte (GOÄ) abrechnen.

\section{Einverständniserklärung}

Ich wünsche die nachfolgend gekennzeichnete Behandlung, z. B.

$\square$ Laserverödung von erweiterten Gefäßen (Teleangiektasien)

$\square$ Entfernung von uncharakteristischen Pigmentflecken und Altersflecken

Entfernung von kleinen Blutgefäß-Gewächsen (Angiomen)

$\square$ Entfernung von Tätowierungen

$\square$ operative Entfernung von kosmetisch störenden, harmlosen Hautgewächsen

$\square$ operative Entfernung von übergroßen Talgdrüsen (Hyperplasien)

- Unterspritzung von Falten, kleinen Narben (Collagen-Implantat)

$\square$

Mir ist bekannt, daß es sich um eine kosmetisch-ästhetische Leistung handelt, die medizinisch nicht notwendig ist und die entstehenden Kosten daher nicht von der Krankenkasse erstattet werden. Ich bin mit der Abrechnung auf der Grundlage der Gebührenordnung für Ärzte (GOÄ) entsprechend den Empfehlungen der Dt. Gesellschaft für ästhetische Dermatologie einverstanden.

Ich wurde über Art und Bedeutung des Eingriffs, über die möglichen unerwünschten Wirkungen wie:

sowie der Möglichkeit von Rezidiven aufgeklärt.

Es werden voraussichtlich Sitzungen erforderlich sein.

Das Honorar pro Sitzung beträgt voraussichtlich Euro

Name

Vorname

Geburtsdatum

Unterschrift des Patienten

Unterschrift des Arztes 


\section{Gynäkologie - Geburtshilfe - Reproduktionsmedizin}

Was bietet die Fachgruppe als IGeL-Leistungen an?

- Gynäkologische Komplett-Vorsorge - Frauen-Gesundheits-Check + Mammographie + MR-Mammographie

- Anti-Aging-Check

- Einlage IUP - IUP-Lagekontrolle außerhalb der GKV-Leistungspflicht

- Einlage Hormonpresslinge)

- Einlage Implanon

- Osteoporose Vorsorge)

- HVP Screening

- Perimenopausen Status

- Ausschlussdiagnostik sexuell übertragbarer Krankheiten

- Sexualberatung

- Sterilitätsvorsorge auf chlamydia trachomonatis

- Ausschluss Infektionen bei Schwangerschaftswunsch

Schwangerschaft

- Schwangerenvorsorge: Zusatzdiagnostik auf Wunsch der Patientin

- Antikörperbestimmung in der Schwangerschaft

- Triple-Test aufWunsch der schwangeren Patientin

- Babyfernsehen

- Geburtsvorbereitung mit Akupunktur

Reproduktionsmedizin

- In-Vitro-Fertilisatio

- intrazytoplasmatische Spermieninjektion

- Spermienuntersuchung bei Verwendung kryokonservierter Hodengewebsproben

\section{IGeL-Angebote einer Frauenarztpraxis mit Preisen}

- Ein niedergelassener Gynäkologie aus Wuppertal http://www.frauenarzt-im-tal.de/Leistungen/ Kosten/kosten.html) integriert in sein IGeL-Angebot gleich mit die für die Patientin zu erwartenden Preise ordnungsgemäß mit den entsprechenden GOÄ Leistungsziffern. Nachfolgend das Angebot in Ausschnitten, das zur Motivation für Gynäkologen dienen dürfte, die IGeL-Leistungen ihren Patientinnen anbieten möchten:

- ... „Das Honorar errechnet sich nach der amtlichen Gebührenordnung für Ärzte (GOÄ), Stand: 1.1.1996.

- Besteht ärztlicherseits kein entsprechender Krankheitsverdacht und keine medizinische Notwendigkeit für diese Leistung, sind diese nicht im Leistungsumfang der Krankenkasse enthalten.

\begin{tabular}{|c|c|c|}
\hline Leistung & GOÄ-Ziffern & Euro \\
\hline $\begin{array}{l}\text { Bescheinigung über die Schwangerschaft für den Arbeitgeber; Reise- } \\
\text { rücktritt }\end{array}$ & 70 & 10 \\
\hline $\begin{array}{l}\text { Bescheinigungen, wie z. B. für den Arbeitgeber, die Schule, den Sport- } \\
\text { verein. } \\
\text { Bescheinigung über SS-Abbruch, SS-Gymnastik, Arbeitsunfähigkeit } \\
\text { (400-EURO-Job) }\end{array}$ & 70 & 5 \\
\hline Fotokopie pro Seite & 96 & 0,50 \\
\hline Ultraschall der Brüste im Rahmen der Krebsvorsorge (Vorsorge plus M) & $418,420 \times 2$ & 45 \\
\hline $\begin{array}{l}\text { Ultraschall von der Scheide bei der Vorsorgeuntersuchung (Vorsorge } \\
\text { plus V) }\end{array}$ & $\begin{array}{l}403,410 \\
420 \times 2\end{array}$ & 30 \\
\hline $\begin{array}{l}\text { Entnahme Krebsabstrich mittels ThinPrep Pap Test (Vorsorge plus Tp) } \\
{[25 €]}\end{array}$ & 1105 & 25 \\
\hline
\end{tabular}


E. Individuelle Gesundheitsleistungen von A - Z

\begin{tabular}{|c|c|c|}
\hline Leistung & GOÄ-Ziffern & Euro \\
\hline $\begin{array}{l}\text { Entnahme HPV high risk Nachweis vom Muttermund (Vorsorge } \\
\text { plus } P \text { ) }[18-38 €]\end{array}$ & $1,298,321$ & 15 \\
\hline Vorsorge plus U (Urin), B (Blutbild), S (Serum) & & 31340 \\
\hline Chlamydien-Nachweis vom Muttermund & $\begin{array}{l}1,1070 \\
4648\end{array}$ & 28 \\
\hline Test auf Blut im Stuhl „rotes Briefchen” (Vorsorge plus H) & 3500 & 6 \\
\hline Immunologischer Test auf Blut im Stuhl (Vorsorge plus Hi) & $3571 \times 2$ & 20 \\
\hline $\begin{array}{l}\text { Knochendichtemessung mit Ultraschall (Osteoporose - Knochen- } \\
\text { bruchrisiko) }\end{array}$ & $1,75,410$ & 40 \\
\hline „Baby-Fernsehen“, Ultraschall; ggf. mit Videodokumentation & 1,415 & 75 \\
\hline 3D/4D-Ultraschall Baby & & $\begin{array}{c}\text { auf } \\
\text { Nachfrage }\end{array}$ \\
\hline $\begin{array}{l}\text { Einlegen einer Spirale (IUP) } \\
\text { Liegedauer: 2-3 Jahre } \\
\text { Multiload@ [27] }\end{array}$ & $\begin{array}{l}1,7,321, \\
403,410, \\
420 \times 2, \\
1070,1075, \\
1091,1096\end{array}$ & 130 \\
\hline $\begin{array}{l}\text { Einlegen einer Hormonspirale (Mirena@) } \\
\text { Liegedauer: bis } 5 \text { Jahre } \\
\text { Mirena } \odot[150]\end{array}$ & $\begin{array}{l}\text { 1, 7, 321, } \\
403,410, \\
420 \times 2, \\
1070,1075, \\
1091,1096\end{array}$ & 175 \\
\hline $\begin{array}{l}\text { Injektion 3-Monats-Spritze } \\
\left.\text { (Sayana Depo-Clinovir }^{\odot} /{ }_{\text {Sayana }}{ }^{\odot}\right) \\
\text { [30 o 35] }\end{array}$ & 252 & 5 \\
\hline $\begin{array}{l}\text { Einlegen eines Stäbchens zur Empfängnisverhütung (Implanon@) } \\
\text { [199] } \\
\text { Liegedauer: bis } 3 \text { Jahre }\end{array}$ & 1, 2421, 490 & 130 \\
\hline Triple-Test [21]; & 3,250 & 30 \\
\hline $\begin{array}{l}\text { Toxoplasmose [18]; Blutgruppe [31]; HIV [15] } \\
\text { kleiner Hormonstatus bzgl. Wechseljahre: FSH \& 17ß-Östradiol [40]; } \\
\text { weitere Laboruntersuchungen auf Nachfrage }\end{array}$ & 1,250 & 15 \\
\hline Scheiden-pH Screening in der Schwangerschaft pro Test & 3714 & 2,50 \\
\hline $\begin{array}{l}\text { Grund-Impfung gegen wiederholte Scheidenentzündungen } \\
\text { (Gynatren@) [66] }\end{array}$ & $1,375 \times 3$ & 27 \\
\hline Akupunktur (zur Schmerzbehandlung oder bei Beckenendlage) & $1,269 a$ & 26 \\
\hline Impfungen bei „Nicht-Kassenleistung“ (zuzüglich Impfstoff) & 1,375 & $21 \#$ \\
\hline Korrektur störender Hautveränderungen (Warzen etc.) & $1,490,2403$ & $30 \#$ \\
\hline HPV-Impfung (zuzüglich Impfstoff) [3x 160] & $1,3,5,375$ & $35-20-20$ \# \\
\hline
\end{tabular}

- Abkürzungen im Text deuten z. B. auf zusätzlichen Untersuchungen (Vorsorge plus):

- V. plus Ultraschalluntersuchung von der Scheide aus [30 EURO]

- M. plus Brustultraschall [45 EURO]

- Tp. plus spez. Krebsabstrich mittels ThinPrep [25+ ca. 25 EURO]

- U. plus Urinuntersuchung (Zucker, Eiweiß, Blut, Bakterien) [3 EURO]

- B. plus kleine Blutuntersuchung (U + Blutsenkung + Blutkörperchen) [13 EURO]

- S. plus große Blutuntersuchung ( $B+12$ Serum-Organwerte) [40 EURO]

- H. plus Stuhlprobe (Test auf verstecktes Blut im Stuhl) [6 EURO]

- I. plus immunologische Stuhlprobe (spez.Test auf verstecktes Blut) [20 EURO]

- P. plus HPV-Nachweis vom Gebärmutterhals [15 EURO + 18 EURO] 
- Alle aufgeführten Beträge sind Annähernd-Werte.- \# je nach Aufwand kann sich ein anderer Betrag ergeben. - Mein Honorar umfaßt die Blutentnahme, eine Befundkopie für Sie und natürlich die Beratung. - Bei allen Laboruntersuchungen erfolgt eine zusätzliche Rechnungsstellung durch das untersuchende Labor, der ca. EURO-Betrag ist zur Information in [Klammern] angegeben. - Bei Medikamenten ist der Preis des Präparates ebenfalls in [Klammern] mit angegeben.

Stand: November 2010. Änderungen hierbei sind möglich.

- Die Durchführung obiger Leistungen erfolgt auf eigenen Wunsch.

Vorher wird abgeklärt, dass ärztlicherseits kein entsprechender Krankheitsverdacht besteht.

Die Rechnung nicht bei der Krankenkasse zur Kostenerstattung eingereicht werden kann, da es sich um eine Leistung entsprechend SGB V § 12, Satz 2, handelt (Gesetzestext: „Die Leistungen müssen ausreichend, zweckmäßig und wirtschaftlich sein; sie dürfen das Maß des Notwendigen nicht überschreiten. Leistungen, die nicht notwendig oder unwirtschaftlich sind, können Versicherte nicht beanspruchen, dürfen die Leistungserbringer nicht bewirken und die Krankenkassen nicht bewilligen").

\section{Operative Leistungen}

\section{Intimchirurgie der Frau}

Dieser Bereich hat in den letzten Jahren sprunghaft zugenommen. Die Süddeutsche Zeitung schreibt im Juli 2013 unter dem Titel Schamlippen verkleinern, bitte - Lange Zeit akzeptierten sich die Deutschen wenigstens untenrum so, wie sie nun mal waren. Damit ist es vorbei. Nun verschönern Chirurgen auch Genitalien - bei Frauen und bei Männer.

Wer im Internet nach der Intimchirurgie sucht, findet nachfolgende Angebote. Wer an den Behandlungskosten Interesse hat, findet nur Pauschalpreise ausgewiesen.

Schamlippenkorrektur

Schamlippenverkleinerung - Schamlippenvergrößerung

Vaginalstraffung

Ästheitische Operationen nach Geburtsfolgen

Venushügelkorrektur

G-Punkt-Intensivierung

Hymenrekonstruktion

Das Institut Sensualmedics® Munich Medical Center (http://www.sensualmedics.com/de/service/kosten-und-preise.html) in München bietet nicht nur seinen Leistungskatalog im Netz an, sondern gibt auch Preise an:

\section{Schamlippenkorrekturen}

\begin{tabular}{|l|c|}
\hline $\begin{array}{l}\text { Schamlippenverkleinerung der inneren Schamlippen ausschließlich unterhalb der } \\
\text { Klitoris }\end{array}$ & $1.900 €$ \\
\hline Schamlippenverkleinerung der inneren Schamlippen gesamt (sowohl Klitoris-Mantel & \\
\hline als auch unterhalb der Klitoris) & $3.100 €$ \\
\hline Schamlippenverkleinerung der inneren Schamlippen einschließlich Klitorismantel & \\
\hline mit Verlagerung der Klitoris näher zum Vaginaleingang & $3.800 €$ \\
\hline Schamlippenverkleinerung der äußeren Schamlippen & $2.800 €$ \\
\hline Volumenformung (Lipostructure) der äußeren Schamlippen & $2.400 €$ \\
\hline
\end{tabular}

(Eine Verkleinerung der äußeren und inneren Schamlippen ist nicht in einer Operation möglich.)

\section{Vaginalstraffung}

\begin{tabular}{|l|l|}
\hline Scheidenverjüngung durch Eigenfett & $2.700 €$ \\
\hline Scheidenverjüngung durch Gewebe- und Muskelstraffung & \\
\hline mit Beckenbodenplastik und Eigenfett & $7.500 €$ \\
\hline $\begin{array}{l}\text { Bei den stationären Eingriffen fallen zusätzliche Kosten für Narkose und } \\
\text { Klinik an }\end{array}$ & $1.500 €$ \\
\hline
\end{tabular}


E. Individuelle Gesundheitsleistungen von A - Z

Package (z. B. nach Mutterschaften)

Kleine Unterbauchstraffung, Liposuktion Venushügel und Scheidenverengung durch Gewebestraffung $9.000 €$

G-Punkt Intensivierung (G-Shot)

G-Punkt-Intensivierung durch Lipostructure (Eigenfett)

G-Punkt-Intensivierung durch Hyaluronsäure

$2.400 €$

Hymenrekonstruktion

Hymenrekonstruktion (Wiederherstellung des Jungfernhäutchens)

$2.200 €$

\section{Venushügelkorrekturen}

Modellierung des Venushügels durch Liposuktion (Fettabsaugung)

Modellierung des Venushügels durch Lipostrukture (Aufbau durch Eigenfett)

$2.000 €$

Häufige zusätzliche Krebs-Vorsorgeuntersuchungen für Frauen

Die nachfolgend aufgelisteten GOÄ-Leistungen sind ggf. um zusätzliche Untersuchungen (GOÄ Nrn. 7,8, 29) und/oder Beratungen (GOÄ Nr. 3, 34) zu erweitern, wenn für die Krebsvorsorgeuntersuchung auf Patientinnenwunsch nicht die Nr. 27 angesetzt wird.

\begin{tabular}{|c|c|c|c|}
\hline GOÄ Nr. & Kurzlegende & 1 fach $€$ & $\begin{array}{r}{ }^{*} 1,8 / \\
2,3 \text { fach } €\end{array}$ \\
\hline 410 & Ultraschalluntersuchung der Gebärmutter & 11,66 & 26,81 \\
\hline $420(2 x)$ & Ultraschalluntersuchung der Ovarien rechts und links & $4,66(2 x)$ & $10,72(2 x)$ \\
\hline 420 & Ultraschalluntersuchung ein weiteres Organ & 4,66 & 10,72 \\
\hline $403^{*}$ & Transvaginale Ultraschalluntersuchung & 8,74 & 15,74 \\
\hline \multirow[t]{2}{*}{$250^{*}$} & Blutentnahme & 2,33 & 4,20 \\
\hline & Labor & 1 fach $€$ & *1,15fach $€$ \\
\hline $3508^{\star \star}$ & Nativpräparat Vaginalsekret & 4,66 & 5,36 \\
\hline $3550^{\star *}$ & Blutbild & 3,50 & 4,02 \\
\hline $3551^{\text {** }}$ & Differential Blutbild & 1,17 & 1,34 \\
\hline $3511^{* *}$ & Urin-Teststreifen (nicht neben Nr. 27) & 2,91 & 3,35 \\
\hline $3500^{*}$ & Stuhl auf Blut, 3mal & 5,25 & 6,03 \\
\hline
\end{tabular}

Früherkennung Mammakarzinom

Mammographie und/oder Ultraschall der Brust zur Früherkennung des Mammakarzinoms bei Frauen ohne relevante Risikofaktoren auf besonderen Wunsch der Frau Als alleinige Untersuchungsmethode hat die Ultraschalluntersuchung nicht die Aussagekraft einer Mammographie. Aber Ultraschall und Mammographie ergänzen sich.

Indikationen zum Einsatz der Mammasonographie sind

- Kontrolle unklarer Tastbefunde

- mammographisch darstellbare, aber nicht sicher beurteilbare Veränderungen

- Unterscheidung homogener oder zystischer Tumor

- sonographisch gesteuerte Tumorpunktion

- Nachkontrolle nach Mamma-OP, z. B. Einsatz der farbkodierten Sonographie zur Darstellung auffälliger Gefäßmuster 


\begin{tabular}{|l|l|r|r|}
\hline GOÄ Nr. & Kurzlegende & $\mathbf{1 f a c h ~} \mathbf{€}$ & $\begin{array}{r}\text { *1,8/ } \\
\mathbf{2 , 3 f a c h} \boldsymbol{€}\end{array}$ \\
\hline $\mathbf{5 2 6 5}^{*}$ & Mammographie einer Seite, in einer Ebene & 17,49 & $\mathbf{3 1 , 4 8}$ \\
\hline $\mathbf{5 2 6 6}^{*}$ & Mammographie einer Seite, in zwei Ebenen & 26,23 & $\mathbf{4 7 , 2 1}$ \\
\hline $\mathbf{5 2 6 7 ^ { * }}$ & $\begin{array}{l}\text { Ergänzende Ebene(n), Spezialprojektion(en), im Abschluss an } \\
\text { Nr. 5266 }\end{array}$ & 8,74 & $\mathbf{1 5 , 7 4}$ \\
\hline $\mathbf{4 1 8}$ & Ultraschalluntersuchung einer Brustdrüse z. B. rechts & 12,24 & $\mathbf{2 8 , 1 5}$ \\
\hline $\mathbf{4 2 0}$ & + Ultraschalluntersuchung linke Mamma & 4,66 & $\mathbf{1 0 , 7 2}$ \\
\hline
\end{tabular}

\section{MR-Mammographie}

Zahlreiche radiologische Praxen bieten eine MR-Mammographie als IGEL-Leistung an, um der Patientin zusätzliche Sicherheit zu geben, dass eine Krebserkrankung unwahrscheinlich ist. Ein Gynäkologe, der seinen Patientinnen diese Untersuchung zusätzlich anbieten möchte, sollte mit einem radiologischen Kollegen kooperieren.

\begin{tabular}{|l|l|r|r|}
\hline GOÄ Nr. & Kurzlegende & $\mathbf{1}$ fach $\boldsymbol{€}$ & $\begin{array}{r}{ }^{*} \mathbf{1 , 8} / \\
\mathbf{2 , 3 f a c h} \boldsymbol{\ell}\end{array}$ \\
\hline $\mathbf{1}$ & Beratung & 4,66 & $\mathbf{1 0 , 7 2}$ \\
\hline $\mathbf{5}$ & körperliche Untersuchung (Tastbefund) & 4,66 & $\mathbf{1 0 , 7 3}$ \\
\hline $\mathbf{5 7 2 1 ^ { * }}$ & MRT der Mammae (Brust) & 233,15 & $\mathbf{4 1 9 , 6 7}$ \\
\hline $\mathbf{5 7 3 1}^{*}$ & ergänzende Serie nach i.v. Kontrastmittel & 58,29 & $\mathbf{1 0 4 , 9 2}$ \\
\hline $\mathbf{5 7 3 3}^{*}$ & computergestützte Analyse, Kinetik - nur 1facher Satz möglich & $\mathbf{4 6 , 6 3}$ & - \\
\hline $\mathbf{3 4 6}$ & Hochdruck-Kontrastmittel-Injektion & 17,49 & $\mathbf{4 0 , 2 2}$ \\
\hline
\end{tabular}

\section{Kernspintomographie in der Brustkrebs-Diagnostik}

In der Tumordiagnostik des Mammacarc. wird in den letzten Jahren öfter die MRT eingesetzt, um so informiert die Deutsche Krebsgesellschaft (http://www.krebsgesellschaft.de/pat_ka_brustkrebs_diagnose_mrt,114334.html)

... „Informationen über Lage und Größe eines Tumors zu gewinnen. Aufgrund des oftmals unterschiedlichen Wasserstoffgehaltes ist eine Unterscheidung zwischen bösartigem und gesundem Gewebe möglich. Die Kernspintomographie entwickelt sich mehr und mehr zu einem wichtigen Zusatzverfahren auch in der Brustkrebs-Diagnostik. Sie wird insbesondere bei speziellen Fragestellungen eingesetzt:

- Ausschluss sehr kleiner, in der Mammographie nicht sichtbarer Geschwüre bei einem bereits bekannten Tumor

- Kontrolle der Tumorentwicklung während einer Therapie

- Unterscheidung zwischen Narbengewebe nach einer Brustoperation und einem neu aufgetretenen Tumor (Rezidiv)

- Untersuchung von Frauen mit Brustimplantaten

- Früherkennungsuntersuchung bei Patientinnen mit hohem Risiko aufgrund familiär gehäuftem Brust- und/oder Eierstockkrebs

Bisher zahlen die Krankenkassen die Brust-MRT nur in Ausnahmefällen, auch weil das Verfahren wesentlich teurer ist als die Mammographie. Außerdem ist die MRT sehr empfindlich, aber nicht spezifisch genug, $d$. h. durch MRT erkannte Veränderungen müssen nicht unbedingt bösartig sein, können also zu unnötigen Zusatzuntersuchungen führen. Nur bei jungen Frauen mit einem erblichen. stark erhöhten Krebsrisiko wird die Kernspinuntersuchung zur gesetzlichen Früherkennung angewandt, da in diesem Alter die Mammografie wegen des dichten Drüsengewebes oft nicht sehr aussagekräftig ist. Zudem soll gerade diesen Patientinnen jede unnötige Strahlenbelastung erspart werden.

Ob die Kernspintomografie so starke Vorteile gegenüber derzeit üblichen Untersuchungen hat, dass sie in Zukunft routinemäßig für alle Frauen in der Brustkrebs-Diagnostik eingesetzt werden kann, wird noch in Studien untersucht...

\section{... Kernspintomographie zur Früherkennung}

Kernspinuntersuchungen werden nicht routinemäßig zur Früherkennung von Brustkrebs eingesetzt. Ausnahme: Bei Frauen mit einem erblichen stark erhöhten Krebsrisiko beginnt das Früherken- 


\section{E. Individuelle Gesundheitsleistungen von A - Z}

nungsprogramm oft schon mit 25 oder 30. In diesem Alter ist die Mammographie wegen des dichten Drüsengewebes oft nicht sehr aussagekräftig, daher wird hier die Kernspinuntersuchung angewandt. Zudem soll gerade diesen Patientinnen jede unnötige Strahlenbelastung erspart werden..." Kernspintomographie wird von zahlreichen niedergelassenen Radiologen und von Radiologischen Abteilungen von Krankenhäuser - besonders von Universitätskrankenhäusern als das „sicherste Verfahren" zum Früherkennen von Brustkrebs angeboten.

\section{Endometriumkarzinom}

Sonographische Bestimmung der Endometriumdicke zur Früherkennung des Endo-metriumkarzinoms. In der Regel wird der gesamte Unterbauch untersucht, so dass ein zusätzlicher Ansatz der GOÄ-Nr. 420 stattfindet.

\begin{tabular}{|l|l|r|r|}
\hline GOÄ Nr. & Kurzlegende & $\mathbf{1 f a c h} €$ & $\mathbf{2 , 3 f a c h} €$ \\
\hline $\mathbf{4 1 0}$ & Ultraschalluntersuchung eines Organs & 11,66 & $\mathbf{2 6 , 8 1}$ \\
\hline $\mathbf{4 2 0}$ & Ultraschalluntersuchung bis zu 3 weiteren Organen, je Organ & 4,66 & $\mathbf{1 0 , 7 2}$ \\
\hline
\end{tabular}

Früherkennung des Zervixkarzinoms - HPV-Screening

Optimierte zytologische Diagnostik mittels Flüssigkeits-Zytologie (z. B.Thin-Prep, Autocyte). Ggf. laborchemische Untersuchung auf Humane Papilloma-Viren (HPV-Test) zur Abklärung eines erhöhten Risikos für die Entwicklung eines Zervixkarzinoms.

Der Test ist nicht im Krebsfrüherkennungsprogramm aufgenommen und wird auch nicht von den Kassen bezahlt.

\begin{tabular}{|c|c|c|c|}
\hline GOÄ-Nr. & Kurzlegende & 1 fach $€$ & $\begin{array}{r}{ }^{*} 1,15 / \\
2,3 f a c h €\end{array}$ \\
\hline 1 & Beratung, auch tel. & 4,66 & 10,73 \\
\hline 3 & $\begin{array}{l}\text { Eingehende Beratung, auch tel. (mind. } 10 \text { Min.) nicht neben Son- } \\
\text { derleistungen }\end{array}$ & 8,74 & 20,11 \\
\hline 297 & $\begin{array}{l}\text { Entnahme und Aufbereitung von Abstrichmaterial zur zytologi- } \\
\text { schen Untersuchung - ggf. einschließlich Fixierung }\end{array}$ & 2,62 & 6,03 \\
\hline GOÄ-Nr. & Kurzlegende & 1fach $€$ & $* 1,15 €$ \\
\hline $4782^{*}$ & $\begin{array}{l}\text { Enzymatische Transkription von RNA mittels reverser Transkrip- } \\
\text { tase }\end{array}$ & 29,14 & 33,52 \\
\hline $4783^{*}$ & $\begin{array}{l}\text { Amplifikation von Nukleinsäuren o. Nukleinsäurefragmenten mit } \\
\text { Polymerasekettenreaktion (PCR) }\end{array}$ & 29,14 & 33,52 \\
\hline $4784^{*}$ & $\begin{array}{l}\text { Amplifikation von Nukleinsäuren oder Nukleinsäurefragmenten } \\
\text { mit geschachtelter Polymerasekettenreaktion (nested PCR) }\end{array}$ & 58,29 & 67,03 \\
\hline $4785^{*}$ & $\begin{array}{l}\text { Identifizienzng von Nukleinsäurefragmenten durch Hybridisie- } \\
\text { rung mit radioaktiv oder nichtradioaktiv markierten Sonden und } \\
\text { nachfolgender Detektion, je Sonde }\end{array}$ & 17,49 & 20,11 \\
\hline
\end{tabular}

\section{Häufige zusätzliche Untersuchungen bei weiblicher Krebsvorsorge}

Häufig werden im Rahmen der Krebsvorsorge von der Patientin zusätzliche Untersuchungen gewünscht z. B.:

- HIV-Testung

- Osteoporose-Vorsorge Osteodensitometrie (Knochendichtemessung) ggf.

- Laborcheck: Osteoporose-Risiko

- kleines Profil: Knochen-AP, Vitamin D3, Pyridinolin-Crosslinks, Ca, Phosphat

- großes Profil, einschließlich hormonelle Ursachen: Knochen-AP, Calcium, Phosphat, Vitamin D3, Parathormon, Östradiol, Testosteron, Osteocalcin, Pyridinolin-Crosslinks

- Osteoporose-Veranlagung: Untersuchung Vitamin-D-Rezeptortyp 
Gynäkologische Komplett-Vorsorge-,,Frauen-Gesundheits-Check“"

Der „Frauen-Gesundheits-Check“ orientiert sich an der ausgedehnten Krebsvorsorge und bezieht ggf. Mammographie und Sonographie der Mammae mit ein. Zu diesem Wunsch-Check-up der Frau gehören Blutdruckmessung, Blutzuckermessung und auch eine Kontrolle des Impfstatus - ggf. Bestimmung Röteln-Antikörper bei ganz jungen Frauen. Bei z. B. internistisch, dermatologisch oder anderen auffälligen Befunden sollte eine Überweisung zur weiteren Abklärung erfolgen.

\begin{tabular}{|c|c|c|c|}
\hline GOÄ Nr. & Kurzlegende & 1 fach $€$ & $\begin{array}{r}{ }^{*} 1,8 / \\
2,3 f a c h €\end{array}$ \\
\hline 3 & $\begin{array}{l}\text { Eingehende Beratung (mind. } 10 \text { Min.) - nicht neben Sonderleis- } \\
\text { tungen }\end{array}$ & 8,74 & 20,11 \\
\hline 7 & Untersuchung weiblicher Genitaltrakt & 9,33 & 21,45 \\
\hline 410 & Ultraschalluntersuchung der Gebärmutter & 11,66 & 26,81 \\
\hline $420(2 x)$ & Ultraschalluntersuchung der Ovarien rechts und links & $\begin{array}{r}4,66 \\
(2 x)\end{array}$ & $10,72(2 x)$ \\
\hline $403^{*}$ & Transvaginale Ultraschalluntersuchung & 8,74 & 15,74 \\
\hline $5265^{\star}$ & Mammographie einer Seite, in einer Ebene & 17,49 & 31,48 \\
\hline $5266^{*}$ & Mammographie einer Seite, in zwei Ebenen & 26,23 & 47,21 \\
\hline $5267^{*}$ & $\begin{array}{l}\text { Ergänzende Ebene(n), Spezialprojektion(en), im Abschluss an } \\
\text { Nr. } 5266\end{array}$ & 8.74 & 15,74 \\
\hline 418 & Ultraschalluntersuchung einer Brustdrüse z. B. rechts & 12,24 & 28,15 \\
\hline 420 & + Ultraschalluntersuchung linke Mamma & 4,66 & 10,72 \\
\hline \multirow[t]{2}{*}{$250^{*}$} & Blutentnahme & 2,33 & 4,20 \\
\hline & Labor & 1fach $€$ & $\begin{array}{r}1,15 \text { fach } \\
€\end{array}$ \\
\hline $3508^{\star \star}$ & Nativpräparat Vaginalsekret & 4,66 & 5,36 \\
\hline $3550^{* \star}$ & Blutbild & 3,50 & 4,02 \\
\hline $3551^{\star \star}$ & Differential Blutbild & 1,17 & 1,34 \\
\hline $3511^{* *}$ & Urin-Teststreifen (nicht neben Nr. 27) & 2,91 & 3,35 \\
\hline $3560^{*}$ & Glukose & 2,33 & 2,68 \\
\hline $3500^{*}$ & Stuhl auf Blut, 3mal & 5,25 & 6,03 \\
\hline
\end{tabular}

\section{Anti-Aging-Check}

\begin{tabular}{|l|l|r|r|}
\hline GOÄ-Nr. & Kurzlegende & $\mathbf{1}$ fach $€$ & $\begin{array}{r}{ }^{*} \mathbf{1 , 8} / \\
\mathbf{2 , 3 f a c h} €\end{array}$ \\
\hline $\mathbf{1}$ & Beratung, auch tel. & 4,66 & $\mathbf{1 0 , 7 3}$ \\
\hline $\mathbf{3}$ & $\begin{array}{l}\text { Eingehende Beratung, auch tel. (mind. 10 Min.) - nicht neben } \\
\text { Sonderleistungen }\end{array}$ & 8,74 & $\mathbf{2 0 , 1 1}$ \\
\hline $\mathbf{2 5 0}$ & Blutentnahme i.v. & 2,33 & $\mathbf{4 , 2 0}$ \\
\hline GOÄ-Nr. & Kurzlegende & $\mathbf{1 f a c h} €$ & ${ }^{*} \mathbf{1 , 1 5}$ \\
\hline $\mathbf{4 0 2 1}^{*}$ & Follitropin (FSH, follikelstimulierendes Hormon) & 14,57 & $\mathbf{1 6 , 7 6}$ \\
\hline $\mathbf{4 0 3 9}^{*}$ & Östradiol & 20,40 & 23,46 \\
\hline
\end{tabular}

Einlage Kupfer-IUP

\begin{tabular}{|l|l|r|r|}
\hline GOÄ Nr. & Kurzlegende & $\mathbf{1 f a c h} €$ & $\begin{array}{r}{ }^{*} \mathbf{1 , 8 /} \\
\mathbf{2 , 3 f a c h} €\end{array}$ \\
\hline $\mathbf{3}$ & $\begin{array}{l}\text { Eingehende Beratung (mind. 10 Min.) - nicht neben Sonderleis- } \\
\text { tungen }\end{array}$ & 8,74 & $\mathbf{2 0 , 1 1}$ \\
\hline $\mathbf{1}$ & Beratung & 4,66 & $\mathbf{1 0 , 7 3}$ \\
\hline
\end{tabular}


E. Individuelle Gesundheitsleistungen von A - Z

\begin{tabular}{|l|l|r|r|}
\hline GOÄ Nr. & Kurzlegende & $\mathbf{1 f a c h} \boldsymbol{€}$ & $\begin{array}{r}{ }^{* 1,8 /} \\
\mathbf{2 , 3 f a c h} \boldsymbol{€}\end{array}$ \\
\hline $\mathbf{7}$ & Untersuchung weiblicher Genitaltrakt & 9,33 & $\mathbf{2 1 , 4 5}$ \\
\hline $\mathbf{2 9 8}$ & Abstrich & 2,33 & $\mathbf{5 , 3 6}$ \\
\hline $\mathbf{1 0 7 5}$ & Vaginale Behandlung & 2,62 & $\mathbf{6 , 0 3}$ \\
\hline $\mathbf{1 0 9 6}$ & Erweiterung des Gebärmutterhalses & 8,63 & $\mathbf{1 9 , 8 4}$ \\
\hline $\mathbf{1 0 9 1}$ & Einlage oder Wechsel IUP & 6,18 & $\mathbf{1 4 , 2 1}$ \\
\hline $\mathbf{4 1 0}$ & Ultraschall eines Organs & 11,66 & $\mathbf{2 6 , 8 1}$ \\
\hline $\mathbf{4 2 0}$ & Ultraschalluntersuchung bis zu 3 weiteren Organen, je Organ & 9,32 & $\mathbf{2 1 , 4 4}$ \\
\hline $\mathbf{4 0 3}^{*}$ & Zuschlag transcavitäre Untersuchung & 8,74 & $\mathbf{1 5 , 7 4}$ \\
\hline
\end{tabular}

Auslagen: Materialkosten für IUP

Einlage Hormonspirale

Zusätzlich zu den oben aufgeführten Leistungen werden ferner abgerechnet:

\begin{tabular}{|l|l|r|r|}
\hline GOÄ Nr. & Kurzlegende & $\mathbf{1 f a c h} €$ & $\begin{array}{r}{ }^{* 1,8 /} \\
\mathbf{2 , 3 f a c h} €\end{array}$ \\
\hline 253 & Injektion, i.v. & 4,08 & $\mathbf{9 , 3 8}$ \\
\hline 490 & Infiltrationsanästhesie 2 mal & 7,12 & $\mathbf{1 6 , 3 6}$ \\
\hline
\end{tabular}

Auslagen: Materialkosten für Hormonspirale

Einlage Implanon

\begin{tabular}{|l|l|r|r|}
\hline GOÄ Nr. & Kurzlegende & $\mathbf{1 f a c h} €$ & $\mathbf{2 , 3 f a c h} €$ \\
\hline $\mathbf{3}$ & $\begin{array}{l}\text { Eingehende Beratung (mind. 10 Min.) - nicht neben Sonderleis- } \\
\text { tungen }\end{array}$ & 8,74 & $\mathbf{2 0 , 1 1}$ \\
\hline $\mathbf{1}$ & Beratung & 4,66 & $\mathbf{1 0 , 7 3}$ \\
\hline $\mathbf{7}$ & Untersuchung weiblicher Genitaltrakt & 9,33 & $\mathbf{2 1 , 4 5}$ \\
\hline $\mathbf{4 9 0}$ & Infiltrationsanästhesie & 3,56 & $\mathbf{8 , 1 8}$ \\
\hline $\mathbf{2 9 1}$ & Implantation von Hormonpresslingen & 4,08 & $\mathbf{1 4 , 2 8}$ \\
\hline
\end{tabular}

Bei einigen Autoren findet sich statt der Nr. 291 die wesentlich höher bewertete Nr. 2421:

\begin{tabular}{|l|l|r|r|}
\hline GOÄ Nr. & Kurzlegende & $\mathbf{1 f a c h} €$ & $\mathbf{2 , 3 f a c h ~} \mathbf{~}$ \\
\hline $\mathbf{2 4 2 1}$ & $\begin{array}{l}\text { Implantation Medikamentenreservoir - analog Ansatz - GOÄ § 6 (2) } \\
\text { GOÄ Kurztext Nr. 2421: Implantation eines subkutanen, auffüllba- } \\
\text { ren Medikamentenreservoirs }\end{array}$ & 34,97 & $\mathbf{8 0 , 4 4}$ \\
\hline
\end{tabular}

+ Materialkosten nicht vergessen (§ $10 \mathrm{GOÄ)}$

IUP-Lagekontrolle mittels Ultraschall außerhalb der GKV-Leistungspflicht

\begin{tabular}{|l|l|r|r|}
\hline GOÄ Nr. & Kurzlegende & $\mathbf{1 f a c h} €$ & $\begin{array}{r}\text { *1,8/ } \\
\mathbf{2 , 3 f a c h ~} €\end{array}$ \\
\hline $\mathbf{3}$ & $\begin{array}{l}\text { Eingehende Beratung (mind. 10 Min.) - nicht neben Sonderleis- } \\
\text { tungen }\end{array}$ & 8,74 & $\mathbf{2 0 , 1 1}$ \\
\hline $\mathbf{4 1 0}$ & Ultraschall Gebärmutter & 11,66 & $\mathbf{2 6 , 8 1}$ \\
\hline $\mathbf{4 0 3}^{*}$ & Zuschlag transcavitäre Untersuchung & 8,74 & $\mathbf{1 5 , 7 4}$ \\
\hline
\end{tabular}




\section{Osteoporose-Vorsorge}

Bei Frauen in der Postmenopause wie auch Männern ab dem 50. Lebensjahr und Patienten mit bekannten Risikofaktoren wie

- Menopause vor dem 45. Lebensjahr

- einseitige Ernährung mit Defizit an Kalzium und Vitamin D

- Bewegungsmangel

- starkem Nikotin- und Alkoholabusus

- Chronischen Colonerkrankungen

- Verwandten ersten Grades mit Osteoporose in der Anamnese

ist eine Bestimmung des Osteoporose-Risikos auf Patienten-Wunsch sinnvoll. Weitere Informationen und Indikationen finden Sie in diesem Kapitel im Bereich Orthopädie.

Osteodensitometrie (Knochendichtemessung)

\begin{tabular}{|c|c|c|c|}
\hline GOÄ Nr. & Kurzlegende & 1fach $€$ & 1,8 fach $€$ \\
\hline $5380^{*}$ & $\begin{array}{l}\text { Osteodensitometrie von Skelettteilen mit quant. CT oder digitaler } \\
\text { Röntgentechnik }\end{array}$ & 17,49 & 31,48 \\
\hline 5377 & $\begin{array}{l}\text { Zuschlag f. computergesteuerte Analyse, einschl. 3D-Rekon- } \\
\text { struktion }\end{array}$ & 46,63 & - \\
\hline $5475^{\star}$ & $\begin{array}{l}\text { Osteodensitometrie von Skelettteilen mittels Dual-Photonen- } \\
\text { Absorptionstechnik }\end{array}$ & 17,49 & 31,48 \\
\hline
\end{tabular}

Abrechnungshinweis: Der Zuschlag nach Nr. 5377 ist nur mit 1 fachem Gebührensatz abrechenbar. Der Zuschlag soll nach Vorschlag des Berufsverbandes der Orthopäden den besonderen Aufwand der Auswertung der Messung berücksichtigen

\section{Laborcheck:}

Osteoporose-Risiko:

- kleines Profil: Knochen-AP, Vitamin D3, Pyridinolin-Crosslinks, Ca, Phosphat.

- großes Profil, einschließlich hormonelle Ursachen: Knochen-AP, Calcium, Phosphat, Vitamin D3, Parathormon, Östradiol, Testosteron, Osteocalcin, Pyridinolin-Crosslinks, Ostase.

Osteoporose-Veranlagung/genetisches Risiko: Vitamin-D-Rezeptortyp.

\section{Perimenopausen Status}

\begin{tabular}{|c|c|c|c|}
\hline GOÄ Nr. & Kurzlegende & 1 fach $€$ & 2,3 fach $€$ \\
\hline 3 & $\begin{array}{l}\text { Eingehende Beratung (mind. } 10 \text { Min.) - nicht neben Sonderleis- } \\
\text { tungen }\end{array}$ & 8,74 & 20,11 \\
\hline \multirow[t]{2}{*}{1} & Beratung & 4,66 & 10,73 \\
\hline & Labor & 1fach $€$ & 1,15 fach $€$ \\
\hline $4021^{*}$ & Follitropin (FSH) & 14,57 & 16,76 \\
\hline 4039* & Östradiol & 20,40 & 23,46 \\
\hline
\end{tabular}

Ausschlussdiagnostik sexuell übertragbarer Krankheiten

\begin{tabular}{|c|c|c|c|}
\hline GOÄ Nr. & Kurzlegende & 1 fach $€$ & $\begin{array}{r}{ }^{*} 1,8 / \\
2,3 \text { fach } €\end{array}$ \\
\hline $31 \mathrm{~A}$ & Ausführliche Sexualanamnese (30 Minuten) - analoger Ansatz & 26,23 & 60,33 \\
\hline 7 & Untersuchung weiblicher Genitaltrakt & 9,33 & 21,45 \\
\hline 27 & Krebsvorsorge (Frau) & 18,65 & 42,90 \\
\hline 29 & Gesundheitssuntersuchung & 25,65 & 58,99 \\
\hline 34 & Erörterung Lebensveränderung (mind. 20 Min.) & 17,49 & 40,22 \\
\hline $250^{*}$ & Blutentnahme venös & 2,33 & 4,20 \\
\hline
\end{tabular}


... „Zu den klassischen Geschlechtskrankheiten gehören Tripper (Gonorrhö), Syphilis (Lues, harter Schanker), weicher Schanker (Ulcus molle) und das Lymphogranuloma venereum, die zusammen jedoch nur etwa 10\% aller Geschlechtskrankheiten ausmachen.

Als sexuell übertragbare Krankheiten (STD - sexual transmitted diseases) treten darüber hinaus heute Chlamydieninfektionen, Trichomoniasis, Herpes genitalis, Krätze, Genitalwarzen und Aids auf.

Auch andere Krankheiten, wie z. B. Hepatitis B und Candidiasis, können durch Geschlechtsverkehr übertragen werden, sind aber keine ausschließlichen Geschlechtskrankheiten..." (Quelle: www.wissen.de))

Nur Fälle von Syphilis und HIV werden in Deutschland gelistet, da für diese Erkrankungen eine Meldepflicht besteht.

Die Untersuchung auf sexuell übertragbare Erkrankungen (STD) sollte entsprechend der Anamnese die Erreger folgender Krankheiten erfassen:

Chlamydien (bakterielle Infektion)

Gonorrhoe (Tripper) (bakterielle Infektion)

Hepatitis B oder Hepatitis C (virale Infektion)

Herpes genitalis (virale Infektion)

HIVIAIDS (virale Infektion)

Humanes Papillomavirus (HPV) (virale Infektion)

Pilzerkrankungen z. B. Candida-Mykose

Syphilis (Lues) (bakterielle Infektion)

Trichomonaden (Parasiten)

\section{Sterilitätsvorsorge auf Chlamydia trachomatis}

Als Folge von Chlamydien-Infektionen kann es bei der Frau zu Adhäsionen im Tubenbereich bis zum Tubenverschluss und damit zur Sterilität kommen. Bei Männern sind Verklebungen der Samenkanälchen die Folge der Infektion.

\begin{tabular}{|c|c|c|c|}
\hline GOÄ Nr. & Kurzlegende & 1 fach $€$ & $2,3 f a c h €$ \\
\hline 3 & $\begin{array}{l}\text { Eingehende Beratung (mind. } 10 \text { Min.) - nicht neben Sonderleis- } \\
\text { tungen }\end{array}$ & 8,74 & 20,11 \\
\hline 1 & Beratung & 4,66 & 10,73 \\
\hline & Labor & 1 fach $€$ & $\begin{array}{r}1,15 \text { fach } \\
€\end{array}$ \\
\hline $4780^{*}$ & Isolierung von Nucleinsäuren & 52,46 & 60,33 \\
\hline $4783^{*}$ & Amplifizierung von Nuleinsäuren oder -fragmenten & 29,14 & 33,52 \\
\hline 4785* & Identifizierung von Nuleinsäurenfragmenten & 58,29 & 67,03 \\
\hline
\end{tabular}

Ultraschall des Unterbauches auf Wunsch der Patientin

\begin{tabular}{|l|l|r|r|}
\hline GOÄ Nr. & Kurzlegende & $\mathbf{1 f a c h} €$ & $\begin{array}{r}\text { *1,8/ } \\
\mathbf{2 , 3 f a c h} €\end{array}$ \\
\hline $\mathbf{1}$ & Beratung & 4,66 & $\mathbf{1 0 , 7 3}$ \\
\hline $\mathbf{7}$ & Untersuchung weiblicher Genitaltrakt & 9,33 & $\mathbf{2 1 , 4 5}$ \\
\hline $\mathbf{4 1 0}$ & Ultraschalluntersuchung der Gebärmutter & 11,66 & $\mathbf{2 6 , 8 1}$ \\
\hline $\mathbf{4 2 0}$ & Ultraschalluntersuchung bis zu drei weiteren Organen, je Organ & 4,66 & $\mathbf{1 0 , 7 3}$ \\
\hline $\mathbf{4 0 3}^{\star}$ & Transvaginale Ultraschalluntersuchung & 8,74 & $\mathbf{1 5 , 7 4}$ \\
\hline
\end{tabular}

Ausschluss Infektionen bei Schwangerschaftswunsch

\begin{tabular}{|l|l|r|r|}
\hline GOÄ-Nr. & Kurzlegende & $\mathbf{1 f a c h} €$ & $\begin{array}{r}{ }^{* 1,15 /} \\
\mathbf{2 , 3 f a c h} €\end{array}$ \\
\hline $\mathbf{1}$ & Beratung, auch tel. & 4,66 & $\mathbf{1 0 , 7 3}$ \\
\hline $\mathbf{3}$ & $\begin{array}{l}\text { Eingehende Beratung, auch tel. (mind. 10 Min.) nicht neben } \\
\text { Sonderleistungen }\end{array}$ & 8,74 & $\mathbf{2 0 , 1 1}$ \\
\hline
\end{tabular}




\begin{tabular}{|c|c|c|c|}
\hline GOÄ-Nr. & Kurzlegende & 1 fach $€$ & $\begin{array}{r}{ }^{*} 1,15 / \\
2,3 \text { fach } €\end{array}$ \\
\hline $250^{*}$ & Blutentnahme i.v. & 2,33 & 4,20 \\
\hline GOÄ-Nr. & Kurzlegende & 1 fach $€$ & *1,15€ \\
\hline 4378* & Antikörper - Cytomegalie-Virus (IgG und IgM) & 13,99 & 16,09 \\
\hline 4382* & Antikörper - Hepatitis A-Virus (IgG und IgM) & 13,99 & 16,09 \\
\hline 4384* & Antikörper - Herpes simplex-Virus (IgG und IgM) & 13,99 & 16,09 \\
\hline $4388^{*}$ & Antikörper - Varizella Zoster-Virus (IgG und IgM) & 13,99 & 16,09 \\
\hline $4406^{*}$ & Antikörper- Hepatitis C-Virus & 23,31 & 26,81 \\
\hline $4461^{*}$ & Antikörper - Toxoplasma gondii & 13,41 & 15,42 \\
\hline
\end{tabular}

Schwangerenvorsorge: Zusatzdiagnostik auf Wunsch der Schwangeren

\begin{tabular}{|c|c|c|c|}
\hline GOÄ Nr. & Kurzlegende & 1 fach $€$ & $\begin{array}{r}{ }^{*} 1,8 / \\
2,3 f a c h €\end{array}$ \\
\hline 23 & Erste Vorsorgeuntersuchung in der Schwangerschaft & 17,49 & 40,22 \\
\hline 415 & $\begin{array}{l}\text { Sonographische Untersuchung auf Vitalität des Feten in der } \\
\text { 6.-8. Schwangerschaftswoche }\end{array}$ & 17,49 & 40,22 \\
\hline 415 & $\begin{array}{l}\text { Zusätzliche sonographische Schwangerschaftsuntersuchung auf } \\
\text { Wunsch der Schwangeren bei Nicht-Risiko-Schwangerschaften } \\
\text { („Baby-Fernsehen“) - ggf. einschl. Geschlechtsbestimmung }\end{array}$ & 17,49 & 40,22 \\
\hline 24 & Untersuchung im Schwangerschaftsverlauf & 11,66 & 26,81 \\
\hline \multirow[t]{2}{*}{$250^{*}$} & Blutentnahme & 2,33 & 4,20 \\
\hline & Labor & 1 fach $€$ & *1,15fach $€$ \\
\hline $3982^{*}$ & Blutgruppen-Bestimmung ABO-Merkmale, Rhesusfaktor & 17,49 & 20,11 \\
\hline $3987^{*}$ & Antikörpersuchtest & 8,16 & 9,38 \\
\hline 4395* & HIV - Test & 17,49 & 20,11 \\
\hline $3743^{*}$ & AFP & 14,57 & 16,76 \\
\hline 4024* & $\beta-\mathrm{HCG}$ & 14,57 & 16,76 \\
\hline \multirow[t]{4}{*}{$4027^{*}$} & Östriol & 14,57 & 16,76 \\
\hline & Toxoplasmose & & \\
\hline & Zytomegalie & & \\
\hline & $\begin{array}{l}\text { Triple-Test zur Risikoabschätzung M. Down und Neuralrohrde- } \\
\text { fekt }\end{array}$ & & \\
\hline
\end{tabular}

Triple-Test auf Wunsch der schwangeren Patientin

Bei Schwangere ab 35 Jahren wird dieser Test von der gesetzlichen Krankenkasse bezahlt. Jüngere Schwangere müssen ihn selber zahlen.

\begin{tabular}{|c|c|c|c|}
\hline GOÄ-Nr. & Kurzlegende & 1 fach $€$ & $\begin{array}{r}{ }^{*} 1,15 / \\
2,3 f a c h €\end{array}$ \\
\hline 1 & Beratung, auch tel. & 4,66 & 10,73 \\
\hline 3 & $\begin{array}{l}\text { Eingehende Beratung, auch tel. (mind. } 10 \text { Min.) nicht neben Son- } \\
\text { derleistungen }\end{array}$ & 8,74 & 20,11 \\
\hline $250^{*}$ & Blutentnahme i.v. & 2,33 & 4,20 \\
\hline GOÄ-Nr. & Kurzlegende & 1 fach $€$ & *1,15€ \\
\hline $3743^{*}$ & Alpha-Fetoproteine (AFP) & 14,57 & 16,76 \\
\hline 4024* & Humanes Choriongonadotropin (HCG) & 14,57 & 16,76 \\
\hline $4027^{*}$ & Östriol & 14,57 & 16,76 \\
\hline
\end{tabular}


E. Individuelle Gesundheitsleistungen von A - Z

„Babyfernsehen“

\begin{tabular}{|l|l|r|r|}
\hline GOÄ Nr. & Kurzlegende & $\mathbf{1 f a c h} €$ & $\begin{array}{r}{ }^{*} \mathbf{1 , 8} / \\
\mathbf{2 , 3 f a c h} €\end{array}$ \\
\hline $\mathbf{1}$ & Beratung & 4,66 & $\mathbf{1 0 , 7 3}$ \\
\hline $\mathbf{4 1 5}$ & Ultraschalluntersuchung Mutterschaft VS & 17,49 & $\mathbf{4 0 , 2 2}$ \\
\hline $\mathbf{4 0 3}{ }^{*}$ & Zuschlag bei transcavitärer Untersuchung & 8,74 & $\mathbf{1 5 , 7 4}$ \\
\hline $\mathbf{4}$ & $\begin{array}{l}\text { Gespräch mit Partner - analoger Ansatz Nr. 4 } \\
\text { GOÄ Kurztext Nr. 4: Erhebung der Fremdanamnese... }\end{array}$ & 12,82 & $\mathbf{2 9 , 4 9}$ \\
\hline
\end{tabular}

Geburtsvorbereitungen mit Akupunktur

\begin{tabular}{|l|l|r|r|}
\hline GOÄ Nr. & Kurzlegende & $\mathbf{1 f a c h} €$ & $\mathbf{2 , 3 f a c h} €$ \\
\hline $\mathbf{2 6 9}$ & Akupunktur - analoger Ansatz Nr. 269 & 11,66 & $\mathbf{2 6 , 8 1}$ \\
\hline $\mathbf{2 6 9}$ a & Akupunktur mind. 20 Min. - analoger Ansatz Nr. 269 a & 20,40 & $\mathbf{4 6 , 9 2}$ \\
\hline $\mathbf{4}$ & $\begin{array}{l}\text { Gespräch mit Partner - analoger Ansatz Nr. 4 } \\
\text { GOÄ Kurztext Nr. 4: Erhebung der Fremdanamnese... }\end{array}$ & 12,82 & $\mathbf{2 9 , 4 9}$ \\
\hline
\end{tabular}




\section{Hals-, Nasen-, Ohrenheilkunde}

\section{Was bietet die Fachgruppe als IGeL-Leistungen an?}

\section{Infusionen}

Bei Hörsturz, Tinnitus und Schwindel-erkrankungen zur Verbesserung der Durchblutung des Innenohres und der peripheren Gleichgewichtsorgane

\section{Akupunktur}

Klassische chinesische Akupunktur bei akuten oder chronischen Nasenneben-höhlenerkrankungen, sowie bei Ver-spannungen im Halswirbelsäulenbereich (HWS)

Kombination von Ohr- und Körperakupunktur

\section{Geruchs - Geschmackstest}

Diagnostik mit verschiedenen Geruchs- und Geschmacksstoffen

\section{Kehlkopfkrebsvorsorge}

Lupenlaryngoskopische (spezielle Vergrößerungsoptik) ausgiebige Betrachtung des gesamten unteren Rachens und inneren Kehlkopfs zum Ausschluss bösartiger Neubildungen, besonders bei Nikotin- und regelmäßigem Alkoholgenuss, inklusive Sonografie des Lymphabflussgebietes am Hals

\section{Schilddrüsendiagnostik}

Genaue sonografische Darstellung des Schilddrüsengewebes inklusive möglicher Knoten und/oder Cysten

\section{Schwindeltherapiekurs}

Vorwiegend bei Erkrankungen der peripheren Gleichgewichtsorgane, gezieltes Anti - Schwindeltraining unter Anleitung einer geschulten Arzthelferin

\section{Streptokokken - Schnelltest}

Rachenabstrich, ab 16. Lebensjahr als IGeL - Leistung

Vitalisierungskur

Hochdosis - Infusionstherapie mit Vitamin_C zur Stärkung des Immunsystems

Hörprüfung

\begin{tabular}{|c|c|c|c|}
\hline GOÄ-Nr. & Kurzlegende & 1 fach $€$ & $\begin{array}{r}{ }^{*} 1,15 / \\
2,3 f a c h €\end{array}$ \\
\hline 1 & Beratung, auch tel. & 4,66 & 10,73 \\
\hline 3 & Eingehende Beratung, auch tel. & 8,74 & 20,11 \\
\hline 5 & Symptombezogene Untersuchung & 4,66 & 10,73 \\
\hline 6 & HNO Untersuchung & 5,83 & 13,41 \\
\hline $1403^{*}$ & Tonschwellenaudiometrische Untersuchung, auch beidseitig & 9,21 & 16,58 \\
\hline 1407 & $\begin{array}{l}\text { Impedanzmessung am Trommelfell u./o. an den Binnenohrmus- } \\
\text { keln (z. B. Stapedius-Lautheitstest), auch beidseitig }\end{array}$ & 10,61 & 24,40 \\
\hline 1415 & $\begin{array}{l}\text { Binokularmikroskopische Untersuchung des Trommelfells und/ } \\
\text { oder der Paukenhöhle zwecks diagnostischer Abklärung, als } \\
\text { selbständige Leistung - Kommentar: Nur zur Abklärung krank- } \\
\text { hafter Veränderungen }\end{array}$ & 5,30 & 12,20 \\
\hline
\end{tabular}

Intratympanale Kortikoidtherapie beim Hörsturz

\begin{tabular}{|l|l|r|r|}
\hline GOÄ-Nr. & Kurzlegende & $\mathbf{1 f a c h} €$ & $\begin{array}{r}{ }^{\mathbf{1}} \mathbf{1 , 8} \\
\mathbf{2 , 3 f a c h} €\end{array}$ \\
\hline $\mathbf{1}$ & Beratung, auch tel. & 4,66 & $\mathbf{1 0 , 7 2}$ \\
\hline $\mathbf{3}$ & Eingehende Beratung, auch tel. & 8,74 & $\mathbf{2 0 , 1 1}$ \\
\hline $\mathbf{5}$ & Symptombezogene Untersuchung & 4,66 & $\mathbf{1 0 , 7 3}$ \\
\hline $\mathbf{6}$ & HNO Untersuchung & 5,83 & $\mathbf{1 3 , 4 1}$ \\
\hline
\end{tabular}


E. Individuelle Gesundheitsleistungen von A - Z

\begin{tabular}{|l|l|r|r|}
\hline GOÄ-Nr. & Kurzlegende & $\mathbf{1 f a c h ~} €$ & $\begin{array}{r}\text { *1,8/ } \\
\mathbf{2 , 3 f a c h} €\end{array}$ \\
\hline $\mathbf{1 4 1 5}$ & $\begin{array}{l}\text { Binokularmikroskopische Untersuchung des Trommelfells und/ } \\
\text { oder der Paukenhöhle zwecks diagn. Abklärung, als selbständige } \\
\text { Leistung - Kommentar: Nur zur Abklärung krankhafter Verände- } \\
\text { rungen }\end{array}$ & 5,30 & $\mathbf{1 2 , 2 0}$ \\
\hline $\mathbf{1 5 7 5}$ & Inzision des Trommelfells (Parazentese) & 7,58 & $\mathbf{1 7 , 4 3}$ \\
\hline $\mathbf{4 8 5}$ & Lokalanästhesie des Trommelfells und/oder der Paukenhöhle & 2,68 & $\mathbf{6 , 1 7}$ \\
\hline $\begin{array}{l}\mathbf{2 5 6} \\
\text { analog }\end{array}$ & Intratympanale Injektion - analog GOÄ Nr. 256 & 10,78 & $\mathbf{2 4 , 8 0}$ \\
\hline
\end{tabular}

Kehlkopf-Ca.-Prävention

\begin{tabular}{|l|l|r|r|}
\hline GOÄ-Nr. & Kurzlegende & $\mathbf{1 f a c h} €$ & $\begin{array}{r}{ }^{* 1,8 /} \\
\mathbf{2 , 3 f a c h} €\end{array}$ \\
\hline $\mathbf{1}$ & Beratung, auch tel. & 4,66 & $\mathbf{1 0 , 7 2}$ \\
\hline $\mathbf{3}$ & Eingehende Beratung, auch tel. & 8,74 & $\mathbf{2 0 , 1 1}$ \\
\hline $\mathbf{5}$ & Symptombezogene Untersuchung & 4,66 & $\mathbf{1 0 , 7 3}$ \\
\hline $\mathbf{6}$ & HNO Untersuchung & 5,83 & $\mathbf{1 3 , 4 1}$ \\
\hline $\mathbf{1 5 3 0}$ & Untersuchung des Kehlkopfes m. Laryngoskop & 10,61 & $\mathbf{2 4 , 4 0}$ \\
\hline
\end{tabular}

Tinnitus

\begin{tabular}{|l|l|r|r|}
\hline GOÄ-Nr. & Kurzlegende & $\mathbf{1 f a c h} €$ & $\begin{array}{r}\text { *1,15/ } \\
\mathbf{2 , 3 f a c h} €\end{array}$ \\
\hline $\mathbf{1}$ & Beratung, auch tel. & 4,66 & $\mathbf{1 0 , 7 3}$ \\
\hline $\mathbf{3}$ & Eingehende Beratung, auch tel. & 8,74 & $\mathbf{2 0 , 1 1}$ \\
\hline $\mathbf{5}$ & Symptombezogene Untersuchung & 4,66 & $\mathbf{1 0 , 7 3}$ \\
\hline $\mathbf{2 5 3}$ & Injektion i.v. & 4,08 & $\mathbf{4 , 3 8}$ \\
\hline $\mathbf{2 7 2}$ & Infusion bei Tinnitus 5 x ggf. auch 10 x & 10,49 & $\mathbf{2 4 , 1 3}$ \\
\hline $\begin{array}{l}\mathbf{1 4 0 3} \\
\text { analog }\end{array}$ & Tinnitusfrequenzbestimmung analog GOÄ Nr. 1403 & 9,21 & $\mathbf{1 6 , 5 8}$ \\
\hline
\end{tabular}

Kosten für Infusionslösungen, Infusionsbesteck, und Medikamente nach § 10 GOÄ ansetzbar

Als weitere Therapien finden Anwendung:

- Magnetfeldtherapie

- Sauerstofftherapie nach Ardenne

- Akupunktur

Digitale Volumentomographie (DVT)

Die HNO Praxis Dr. Korbmacher (http://www.hno-korbmacher.de/3d-roentgen-dvt/) aus Düsseldorf informiert über die Methode: ... „Die digitale Volumentomographie (DVT) ist ein modernes dreidimensionales Tomographieverfahren auf der Basis vonRöntgenstrahlen. Ähnlich wie bei der Computertomographie (CT) ermöglicht die DVT die Erzeugung von hochauflösenden Schnittbildern in allen Raumebenen, sowie die Berechnung dreidimensionaler Ansichten. Gegenüber dem CT bietet DVT eine maximale Auflösung bei deutlich geringerer Strahlenexposition.

Die knöchernen Strukturen des Mittelgesichts und der Nasennebenhöhlen können in der DVT ideal beurteilt werden. Anatomische Normvarianten, raumfordernde Veränderungen, Entzündungen und die Lokalisation von Fremdkörpern lassen sich sicher diagnostizieren. Sowohl die Planung, als auch die Ergebnisse chirurgischer Eingriffe an den Nasennebenhöhlen können mit der DVT exzellent dargestellt werden. Selbst das Innenohr lässt sich hochaufgelöst abbilden. 
Die wesentlichen Vorteile für den Patienten:

- Der Patient sitzt aufrecht und muss nicht in einer Röhre liegen.

- Die Technik arbeitet mit einer außerordentlich geringen Strahlendosis. Sie beträgt nur ca. ein 1/10 der Strahlenbelastung eines herkömmlichen CT-Geräts.

- Der Zusammenhang zwischen Zahnwurzeln und Kieferhöhlen kann bestmöglich beurteilt werden.

- Befunde können mit dreidimensionalen Bildern sehr anschaulich dargestellt werden...“

In der Zahnmedizin spricht man inzwischen von der Dentalen Volumentomographie. Im englischsprachigen

\begin{tabular}{|c|c|c|c|}
\hline GOÄ-Nr. & Kurzlegende & 1fach $€$ & $\begin{array}{r}{ }^{*} 1,8 / \\
2,3 f a c h \\
\end{array}$ \\
\hline 1 & Beratung, auch tel. & 4,66 & 10,72 \\
\hline $\begin{array}{l}5370^{\star} \\
\text { analog }\end{array}$ & $\begin{array}{l}\text { Abrechnung der digitalen Volumentomographie - } \\
\text { analog GOÂ Nr. 5370* }\end{array}$ & 116,57 & 209,83 \\
\hline 5377 & $\begin{array}{l}\text { Zuschlag für computergesteuerte Analyse - einschließlich spezi- } \\
\text { ell nachfolgender 3D-Rekonstruktion - nur im } 1 \text { fachen Satz } \\
\text { berechnungsfähig }\end{array}$ & 46,63 & - \\
\hline
\end{tabular}




\section{Laboruntersuchungen auf Patientenwunsch}

Für welche Symptome oder Krankheitsbilder bietet die Fachgruppe der Labormediziner IGeLLeistungen zur Abklärung u. a. an?

- Alkohol-Abhängigkeit?

- Anti-Aging : Einzelanalysen und Analyseprofile

- Risikofaktoren für Arteriosklerose und Thrombose

- Bestimmung des antioxidativen Status

- Vitaminen und Spurenelementen

- Individueller Hormonstatus

- Überprüfung Immunsystem, Fettstoffwechsel, Schwermetallbelastung etc.

- Abklärung spezieller genetischer Risiken

- Arteriosklerose-Risiko

- Blutfette-Basis-Check

- Blutgruppenbestimmung

- Borreliose und FSME

- Chronische Müdigkeit

- Darmkrebsvorsorge: Immunologischer Stuhltest

- Diabetes-Risiko-Check

- Drogenscreening

- Urin-Drogenscreening nachgewiesen werden: Amphetamine, Barbiturate, Benzodiazepine, Kannabis-Metaboliten, Kokain, Methadon, Opiate, Phencyklid

- Dysbiose

- Eisenmangel-Basis-Check

- Fertilitäts-Check

- Gebärmutterhalskrebs-Risiko (HPV-Test)

- Gesundheits-Check-up - weiterführende Untersuchungen

- Haarausfall

- Hämochromatose (Eisenspeicherkrankheit)

- Helicobacter pylori

- Hepatitis C

- Herzinfarkt-Risiko-Check

- Histamin-Intoleranz

- Immunstatus

- Lactose-Intoleranz

- Leber-Risiko-Check

- Metabolisches Syndrom

- MRSA

- Niereninsuffizienz

- Osteoporose-Diagnostik

- Desoxypyridinolin i. Urin - Estradiol (Frau) - Knochen-AP (BAP) - 25-OH-Vitamin D3 - Parathormon

- Oxidativer Stress/Freie Radikale/Antioxidantien

- Prostatakrebs: PSA

- Prostatakrebs: PCA3-Gentest

- Reisemedizin (Impfschutz)

- Rheuma-Risiko

- Schilddrüsen-Check

- Sexuell übertragbare Erkrankungen

- Sterilitätsvorsorge (Chlamydien-Test)

- Thrombose-Risiko

- Basisprofil: Antithrombin - APC-Resistenz (Gerinnungstest) - Protein C - Protein S - Faktor VIIIAktivität

- Erweitertes Profil: Faktor V-Mutation + Prothrombin-Mutation - Homocystein - Lupus Antikoagulans (Suchtest) - Cardiolipin-Antikörper IgG, IgM

- Tumormarker - in diesem Buch siehe Tumormarker zur Verlaufskontrolle und Diagnostik Seite $136 f$.

- Vaterschaftstest 
E. Individuelle Gesundheitsleistungen von A - Z

- Vitalstoff-Check

- Vitamin D

- "Wechseljahre" der Frau

- "Wechseljahre" des Mannes

- Schwangerschaft: Störungen der Erbanlagen: Down-Syndrom

- Schwangerschaft: Immunstatus

- B-Streptokokken - Cytomegalie-Virus, IgG - Parvovirus B 19, IgG - Varicella-Zoster-Virus, IgG - Toxoplasma gondii, IgG

- Schwangerschaft: Pränatal-Screening (Triple-Test)

- Alpha-Fetoprotein (AFP

- Estriol, freies

- Choriongonadotropin

Bei den folgenden unterschiedlichen Laborprofilen haben wir im Interesse einer schnellen Übersicht und Vollständigkeit, sowie wegen des Umfanges von zusätzlichen Erläuterungen bei allen Laboruntersuchungen, auf die jeweiligen GOÄ-Nrn. verzichtet. Die GOÄ-Nrn. der Leistungen, die im Rahmen von Untersuchungen in der Praxis oder in einer Laborgemeinschaft geleistet werden, dürften hinreichend bekannt sein. Leistungen, die vom Laborarzt erbracht werden, liquidiert er auch selber.

Wichtig:

Es ist dringend anzuraten, Leistungen, die nicht selbst bzw. zulässigerweise von Dritten erbracht wurden, nicht zu liquidieren. Im Zweifel sollte der die Leistung - z. B. eine Laborleistung - erbringende Arzt eine eigenständige Rechnung erstellen. Wird ein weiterer Arzt auf Veranlassung des behandelnden Arztes tätig - z. B. ein Laborarzt -, so muss der Patient unterrichtet werden, dass er auch von diesem Arzt eine Rechnung erhält. Ein diesbezüglicher Vermerk in der Patienten-Kartei ist auch aus Datenschutzgründen dringend zu empfehlen.

Dieses Gebot der persönlichen Leistungserbringung wird in der letzten Zeit nicht nur von privaten Krankenkassen genauer überprüft, sondern auch Staatsanwaltschaften sind vermehrt dazu übergegangen, im Zuge von Ermittlungen bei Verdacht von Abrechnungsbetrug diesen Aspekt näher zu durchleuchten.

Bis auf wenige Ausnahmen wurden uns nachfolgenden Laborprofile und die dazugehörigen Hinweise freundlicherweise vom Umweltmedizinischen Labor München zur Verfügung gestellt.

Alle Labore stellen IGeL-Profile zur Verfügung, aus denen Arzt und/oder Patient Leistungen wählen können. In der Regel können auch spezielle Informationsschriften für die Patienten zu verschiedenen Untersuchungen/Symptomeen/Krankheitsbildern angefordert werden sowie Informationszettel für Patienten zu zahlreichen Laboruntersuchungen.

\section{Adipositas - Laborcheck}

Erstuntersuchung - Kurzprogramm: Blutzucker, Cholesterin, LDL/HDL-Cholesterin, Triglyceride, Harnsäure, TSH basal, Cortisol basal, Homocystein.

Erweitertes Programm: Blutzucker, Cholesterin, LDL/HDL-Cholesterin, Triglyceride, Harnsäure, TSH basal, Cortisol basal, Homocystein, Vit B12, Folsäure, HbA1c, Insulin, LP (a); Harnsäure

\section{Alkohol - Abusus chron.?}

Untersuchung zum längerfristigen Trinkverhalten

Bestimmung: Carbohydrat Deficient Transferrin(CDT)

\section{Alopezie-Haarausfall-Risiko}

Für Haarausfall und im extremen Fall Haarlosigkeit gibt es eine Reihe Risikofaktoren, deren rechtzeitige Erkennung zu präventiven und therapeutischen Maßnahmen beitragen kann. Hierzu zählen u. a. hormonelle Störungen wie z. B. Überproduktion von männlichen Sexualhormonen bei Frauen oder von Schilddrüsenhormonen, Ernährungsstörungen wie z. B. Mangel an Vitaminen und Spurenelementen, Krebserkrankungen, Pilzinfekte oder Autoimmunerkrankungen. Intoxikationen, z. B. mit Quecksilber, können ebenfalls zu Haarausfall führen.

Basisprofil „Allgemeines Alopezie-Risiko“: TSH basal, fT3, fT4, Ferritin, Zink, Magnesium, Vitamin E, Vitamin B12, Folsäure, Vitamin A, antinukleäre Faktoren (ANA).

Bei Verdacht auf Quecksilberintoxikation: siehe Dimaval-Test IGEL S 
E. Individuelle Gesundheitsleistungen von A - Z

\begin{tabular}{|l|l|}
\hline \multicolumn{2}{|c|}{ Spezialprofil „Alopezie Risiko“ } \\
\hline bei Frauen & bei Männern \\
\hline Testosteron & Testosteron \\
\hline Dehydroepiandrosteron-Sulfat (DHEAS) & Cortisol \\
\hline Androstendion & LH \\
\hline Östradiol & FSH \\
\hline SHBG & \\
\hline
\end{tabular}

\section{Blutgruppenbestimmung}

- einschl. irregul. Antikörper, Rhesusformel, Untergruppen und Ausweis für Patienten

- Blutgruppenbestimmung, nur A/B/O/Rh-Faktor

\section{Erschöpfungssyndrom - Chronic Fatigue Syndrome (CFS)}

Bei ausgeprägtem Leistungsabfall sowie übermäßiger Erschöpfung nach Belastung - über einen Zeitraum von mindestens 6 Monaten - müssen mögliche Ursachen wie Stoffwechselerkrankungen (Schilddrüse, Nebenniere/Hypophyse, Diabetes mellitus etc.) aber auch chronische Infektionen (Herpesviren, Borrelien, Hepatitis, etc.) ausgeschlossen werden. Die primäre Ursache des CFS ist bis heute unbekannt, so dass der Nachweis auf dem Ausschluss der genannten Ursachen einerseits und der Charakterisierung CFS-typischer Veränderungen andererseits beruht. Hierzu zählen in erster Linie Funktionsveränderungen des Immunsystems (Aktivierung des zellulären Immunsystems, NK-Zelldefekt Immunglobu-linmangel, Allergien, etc.)

- CFS1 (Basisprofil): Blutbild, Eisen, Kupfer, Magnesium, TSH1, Kreatinin, Cortisol, IGF-1 gesamt, DHEAS, EBV-Serologie, Herpesvirus Typ 6, Lyme-Borreliose, Zytokin-sekretion

\section{Dentalmaterial-Unverträglichkeit}

Die moderne Zahnmedizin nutzt ein breites Spektrum an Materialien zur optimalen Versorgung der Patienten. Diese Stoffe haben in der Regel allgemeine Verträglichkeitsprüfungen durchlaufen und bereiten im klinischen Einsatz bei den meisten Personen keine Probleme. Trotzdem können bei empfindlichen Personen Unverträglichkeitsreaktionen gegenüber diesen Materialien (Metalle, dentale Kunststoffe, Bestandteile von Wurzelfüllmaterialien oder Zemente) auftreten. Ursächlich für Beschwerden sind sehr häufig zelluläre Sensibilisierungen (Immunreaktion TypIV), die mittels eines optimierten Lymphozytentransformationstests (LTT) nachgewiesen werden können. Eine chronische Exposition mit Fremdstoffen aus Zahnersatzmaterialien beinhaltet auch das Risiko einer akkumulierenden zellulären Belastung (zellulärer Stress) sowie eine mögliche unerwünschte Veränderung der oralen Mikroökologie mit resultierender Schädigung des Parodonts (Parodontitis).

Profile: - LTTS/Schwermetalle

- LTTQ/Amalgam

- "LTTG/Goldlegierung

- "LTTP/Spezielle Metalle

- "LTTK/Keramik

- "LTTD/Dentalersatzstoffe

- "LTTTI/Titan

- Dentalmaterial-Unverträglichkeit/Erweitertes Profil: HSP-Antikörper, Glutathion (Erythrozyten), Nerven-Antikörper, Malondialdehyd (MDA), TAS, SOD

- Materialanalyse (Legierungen): Wenn die Zusammensetzung von Legierungen, die möglicherweide für eine Immunisierung verantwortlich sind, nicht bekannt ist, können diese als Vorbereitung etwa zum LTT-Test analysiert werden.

Profil: spez. Analyse

\section{Materialunverträglichkeit}

Manche Menschen reagieren aufgrund immunologischer Reaktionen überempfindlich auf Stoffe aus ihrer Umgebung. Mit klassischen Testverfahren ist das problematische Material oft schwer zu testen, da hier mit industriell gefertigten Testsubstanzen gearbeitet werden muss, die in ihrer Zusammensetzung nicht dem wirklichen Allergen entsprechen. Im Gegensatz dazu können mit den beiden LTVerfahren native Proben (z. B. Kosmetika, Textilien, Reinigungsmittel, Kunststoffe, Stäube, Teppiche, Farben) der verdächtigen Materialien getestet werden. Der LTT deckt hierbei eine mögliche zel- 
luläre Sensibilisierung ab, wogegen mit dem LTC4 humorale Formen und Pseudoallergien erfasst werden.

Untersuchung: LTT oder LTC4

Material: für LTT: 2 x Natrium-Heparin-Blut pro Material

für LTC4: 1 x EDTA-Blut/pro Material

+ jeweils eine Probe des verdächtigen Materials

\section{Fettstoffwechsel}

Unter den Risikofaktoren für die koronare Herzkrankheit spielen Fettstoffwechselstörungen eine zentrale Rolle. Für die Früherkennung haben vor allem die Apolipoproteine B und A-1 größere Bedeutung, da sie die Hauptproteine der atherogenen LDL bzw. der antiatherogenen HDL sind. Ebenso ist der Apo B100-Defekt ein Marker für eine Prädisposition für Atherosklerose und KHK.

Nach wie vor hat auch die Bestimmung des HDL-Cholesterins für die Beurteilung des Atheroskleroserisikos eine große Bedeutung.

- Basisscreening: Chol, Tri, HDL, LDL, Lp(a)

- Erweitertes Screening: Risikogruppen: Cholesterin, Trigylceride, Lipidelektropho-rese, Lp(a)

- Erweitertes Screening: Erbliche Fettstoffwechselstörungen - Erweitertes Profil (bei Risikogruppen, familiärer Belastung): LDL Rezptor-Defekt, Apo B100-Defekt, Apo E

Siehe auch: Laborcheck bei Adipositas

Gesundheitscheck - Altersgruppenspezifisches Labor auf Patientenwunsch

Männer bis 45 Jahre: Basis-Profil: Blutzucker, Harnsäure, GOT, GPT, GGT, Kreatinin, Natrium, Kalium, Calcium, CRP, Elektrophorese, Cholesterin, Triglyceride, TSH, Ferritin, Bilirubin, Amylase, Lipase; Kleines Blutbild, Gesamteiweiß, Urin-Status,

Männer über 45 Jahre: Basis-Profil + zusätzlich: PSA, Hämoglobin/Haptoglobin im Stuhl (Blut im Stuhl, hochsensitive Methode)

Frauen bis 45 Jahre: Basis-Profil + zusätzlich: Ferritin, APC-Resistenz

Frauen über 45 Jahre: Basis-Profil + zusätzlich: Ferritin, Alkalische Phosphatase, CA 15-3, Pyridinolin-Crosslinks im Urin

Zusätzliche Empfehlung Hormonstatus: LH, FSH, DHEAS, Östron, Östradiol, Testosteron, HGH, IGF 1

\section{Zusätzliche Empfehlung Thromboserisiko}

Profil: Protein C, Protein S, APC-Resistenz, AT III, Phospholipid-Antikörper

HIV-Test/anonym

HIV-Antikörperbestimmungen

Hormonstatus im Alter, Klimakterium, Klimakterium virile

Profil bei Männern: LH, freies Testosteron, Progesteron, Östradiol, DHEAS, HGH, IGF 1, TSH

Profil bei Frauen: LH, FSH, DHEAS, Östron, Östradiol, Testosteron, HGH, IGF 1

\section{- Immunologie: Immunstatus - Infektanfälligkeit}

Das Immun- und Abwehrsystem soll den Organismus gegen Bakterien, Viren, Giftstoffe und fremde Eiweißkörper schützen. Wichtige Komponenten des Immunsystems sind im Blut erfassbar, so z. B. die weißen Blutkörperchen, Immunglobuline und weitere Resistenzfaktoren wie z. B. Komplement. Blutzellen wie Lymphozyten und Granulozyten vernichten Krankheitserreger, Immunglobuline binden und eliminieren Fremdstoffe (Antigene) im Blut. Immundefekte können angeboren oder häufiger als Folgen von Erkrankungen und deren Therapie vorkommen, z. B. durch Virusinfekte (HIV), Krebserkrankungen, Zytostatika, Strahlentherapie, Stress oder Umwelteinflüsse (Umweltgifte, Strahlung).

Grundprofil häufige bakterielle Infekte:

Großes Blutbild, IgG quant, IgA quant, IgM quant, sekretorisches IgA, Gesamtkomplement

Ergänzungsprofil häufige bakterielle Infekte:

Phagocytose-Test, Oxidativer Burst

Profil bei häufigen viralen Infekten: T-Zell-Immunkapazität, Lymphocyten-Typisierung 
E. Individuelle Gesundheitsleistungen von A - Z

Siehe auch: Nahrungsmittel-Unverträglichkeit

Nahrungsmittelallergie-Screening IgE/lgG

Siehe auch: Entzündliche Darmerkrankungen

Siehe auch: Zöliakie - panel

\section{- Immuncheck (Funktionstest)}

Dieser Funktionstest für das zelluläre Immunsystem ist geeignet zur Charakterisierung der Immunitätslage (Infektanfälligkeit) sowie für immunologisches Monitoring z. B. bei immun-stimulatorischer Therapie zur individuell optimalen Dosierung.

Profil: Mitogen-Antigen-Stimulation (LTT mit Stimulation durch PWM, PMA, ConA, anti-CD3-Antigen)

\section{Immunstatus}

Das Immunsystem umfasst mehrere zelluläre und humorale Komponenten, die ein komplexes reguliertes Netzwerk bilden. Dabei unterscheidet man zwischen unspezifischen (z. B. Akut-Phase-Proteine, Komplement) und spezifischen Komponenten (Antikörper, Immunzellen). Immundefekte können sowohl angeboren (primär) als auch erworben (sekundär) vorkommen. Sekundäre Defekte sind weitaus häufiger, z. B. nach Virusinfekten, medikamentöser Therapie, Stress, Stoffwechselerkrankungen, Krebserkrankungen oder durch Umwelteinflüsse (Belastung mit toxischen Substanzen, UVStrahlung).

Profil: IgA, IgG, IgM, IgE, CRP, Blutbild, T-Zell-Immunkapazität

\section{Leber - Profil}

Auf Patientenwunsch werden beim Hausarzt häufig Leber-Tests gefordert, obwohl keine Erkrankung vorliegt und damit die Tests nicht im Rahmen der GKV abrechnungsfähig sind.

Großes Leberprofil: Alkalische Phosphatase, Bilirubin gesamt, Gamma-GT, GLDH, SGOP, SGOT, Serum-Elektrophorese einschl. Gesamteiweiß

\section{Medikamenten-Unverträglichkeit (Typi/TypIV-Allergie)}

Die Entwicklung neuer Arzneimittel zu therapeutischen, diagnostischen und prophylaktischen Zwecken ist häufig mit dem Risiko unerwünschter Unverträglichkeitsreaktionen assoziiert. Man geht heute davon aus, dass ca. $5 \%$ der stationären Aufnahmen aufgrund von Arzneimittel-Unverträglichkeiten erfolgen und 15-30\% aller hospitalisierten Patienten im Laufe ihrer Behandlung allergische Sensibilisierungen entwickeln. Nicht nur die Immunogenität des Arzneimittels, dessen Dosierung, Applikation und Therapiedauer spielen bei der Manifestation einer Medikamentenallergie eine Rolle, sondern auch patientenspezifische Besonderheiten in der Immunregulation, immungenetische Einflüsse und individuelle Unterschiede in der Fremdstoffmetabolisierung. Da sowohl humorale als auch zelluläre Reaktionen auftreten können und die Ausprägung des klinischen Bildes auf mehreren pathogenetischen Mechanismen basieren kann, ist eine Zuordnung mancher durch Arzneimittel ausgelöster Krankheitsbilder oft schwierig. Heute stehen für die Diagnostik neben Anamnese, klinischem Befund, Hauttestung und risikobehafteten Provokationstestungen hochsensitive und spezifische In-vitro-Testverfahren zur Verfügung.

Profil: LTT, LTC4: Testung auf die entsprechende Reinsubstanz oder das Präparat (beilegen!)



\section{- Nahrungsmittel-Unverträglichkeit}

Die Prävalenz atopischer Erkrankungen nimmt in den letzten Jahrzehnten in nahezu allen Industrieländern zweifelsfrei zu. Neben den inhalativen Allergien gewinnen Nahrungsmittel-Unverträglichkeiten zunehmend an Bedeutung. Gründe hierfür sind u. a. erweiterte Nahrungsangebote (z. B. Verzehr exotischer Früchte, Gemüse etc.), Einsatz gentechnisch hergestellter Nahrungsmittel und der Trend zu Fertiggerichten. Vor allem die darin enthaltenen Zusatzstoffe entpuppen sich immer häufiger als Auslöser allergischer Reaktionen. Neben toxischen Reaktionen (z. B. Intoxikation durch Giftpilze, Fischvergiftungen durch verdorbenen Fisch) können IgE-vermittelte bzw. (nicht IgE-vermittelte) pseudoallergische Immunantworten induziert werden. Das klinische Bild ist meist von gastrointestinalen, kutanen, respiratorischen oder neurologischen Symptomen geprägt. Auch anaphylaktische Reaktionen wurden beschrieben. Da die auslösenden Allergene und die klinischen Manifestationen nicht immer direkt in Zusammenhang zu setzen sind, wird eine stufendiagnostische Vorgehensweise favorisiert. 


\section{Nahrungsmittelallergie-Screening IgE/lgG}

Basis-Profil: (44 Allergene) Schwein, Rind, Huhn, Forelle, Lachs, Thunfisch, Hering, Kabeljau, Miesmuschel, Weizenmehl, Roggenmehl, Gerstenmehl, Sojabohne, Reis, Gluten, Tomate, Karotte, Kartoffel, Petersilie, Selleriewurzel, Knoblauch, Erdbeere, Zitrone, Orange, Weintraube, Apfel, Pfirsich, Pfeffer, Paprika, Majoran, Erdnuss, Walnuss, Haselnuss, Kuhmilch, Laktalbumin, Laktoglobulin, Gouda, Camembert, Schweizer Käse, Bäckerhefe, Bierhefe, Hühnerei, Kaffee, Schwarzer Tee, Kakao

Erweitertes Profil: Intestinale Permeabilität (IP), LTC4-Panel, sIGA

Vollprofil: LTT, LTC4, Intestinale Permeabilität

\section{Osteoporose-Risiko}

Auch die Knochensubstanz unterliegt einem ständigen Stoffwechsel, d. h. kontinuierlichen Auf- und Abbauvorgängen, die durch den Hormonhaushalt, den Vitaminhaushalt und natürlich den Mineralhaushalt des Körpers beeinflusst werden.

Als Osteoporose bezeichnet man eine Verringerung der Bälkchenstruktur der Knochen (Spongiosa) und eine gleichzeitige Verschmälerung der kompakten Knochenröhre. Die Diagnose von bereits eingetretenen strukturellen Veränderungen des Knochens erfolgt mittels der Knochendichtemessung. Laboruntersuchungen ermöglichen demgegenüber eine frühzeitige Aussage über den Knochenstoffwechsel, wobei Knochenaufbau- und Knochenabbaumarker in Beziehung gesetzt werden und so Verschiebungen des Stoffwechselgleichgewichts erkannt werden können. Die Laboruntersuchungen sind auch eine hervorragende Möglichkeit, den Erfolg einer antiosteoporotischen Therapie zu kontrollieren.

Osteoporose-Risiko/kleines Profil: Knochen-AP, Vitamin D3, Pyridinolin-Crosslinks, Ca, Phosphat

Osteoporose-Risiko/großes Profil, einschließlich hormonelle Ursachen

Knochen-AP, Calcium, Phosphat, Vitamin D3, Parathormon, Östradiol, Testosteron, Osteo-calcin, Pyridinolin-Crosslinks

Osteoporose-Veranlagung: Untersuchung: Vitamin-D-Rezeptortyp

Oxidativer Stress und Entgiftung - Profile zur Diagnose

Mit diesen Untersuchungen kann eine vorhandene Belastung durch reaktive Sauerstoffverbindungen (ROS) und Schadstoffe, der Grad der Zerstörung von genetischem Material und Lipiden einerseits sowie die Kapazität körpereigener (endogener) und zugeführter (exogener) antioxidativer Schutzmechanismen gegen ROS andererseits ermittelt werden. Anhand der Ergebnisse kann eine gezielte antioxidative, nutritiv protektive Therapie geplant werden.

Oxidativer Stress/Mini-Screen

Profil: TAS (gesamte antioxidative Kapazität), SOD (Superoxiddismutase), 8-OhdG, MDA

Oxidativer Stress/Basisprofil

Profil: GSH-ery, GST-pi. TAS, SOD, 8-OhdG, MDA, Vitamin E

Oxidativer Stress/Schutz und Entgiftung

Profil: GST-pi, GST-alpha, Carotenoide, Alpha-Tocopherole, Gamma-Tocopherole, GR. HNE

Oxidativer Stress/Vollprofil

Profil: GSH in Ery, GSSG in Ery (GSH/GSSG Quotient), GST-mu, TAS, Ubichinon. SOD, GPX, MDA, 8-OhdG, Vitamin E

Sport und oxidativer Stress:

Profil: GSH, 8-OhdG (Urin), MDA; Carnitin; SOD, GSH in Erytrozythen, Myogobin, LDH, Isoenzyme

Detoxifikations-Fähigkeit (Raucher, fragl. Umweltbelastung)

Die Fähigkeit des Organismus, mit dauernden Belastungen durch Substanzen aus der Umwelt fertig zu werden (z. B. Schadstoffe, UV-Licht, Schwermetalle, radikale Sauerstoffverbindungen), ist abhängig von der Aktivität verschiedener entgiftender Enzyme und der Konzentration sog. Radikalfänger, Scavenger-Substanzen, die oxidierende Substanzen neutralisieren. Sowohl die Enzymaktivität als auch die Konzentrationen von Scavenger-Substanzen lässt sich quantifizieren.

Außerdem kann mit einigen Markern das Risiko einer organspezifischen Schädigung (z. B. Lunge bei Rauchern) eingeschätzt werden.

Profil: NAT 2, CYP 1A1, NSE, M2PK, Alpha-1-Antitrypsin, Cadmium 


\section{Parodontitis-Diagnostik}

Die chronische Entzündung des Zahnhalteapparats (Parodontitis) ist eine Erkrankung, die von einer Gruppe von hochaggressiven Bakterien hervorgerufen wird. Sie wirkt sich nicht nur lokal, also im Mundraum, sondern auch im Gesamtorganismus schädigend aus. Man weiß, dass Personen mit chronischer, ausgedehnter Parodontitis ein weit höheres Risiko haben, eine Thromboembolie (Herzinfarkt, Schlaganfall) zu erleiden als Personen mit gesundem Zahnfleisch.

Außerdem besteht bei Schwangeren mit Parodontitis ein stark erhöhtes Risiko für eine Frühgeburt. Um diese Risiken zu vermeiden, ist die regelmäßige Kontrolle des Zahnfleisches, die Beseitigung von Plaque und Zahnstein sowie die Behandlung von Zahnfleischentzündungen entscheidend.

Das Labor bietet eine Testung auf das Vorhandensein von Parodontitis verursachenden Bakterien mit individuellem Therapievorschlag für eine Antibiotikabehandlung an. Dieser hoch sensitive und spezifische Test weist diese Keime auch in geringsten Mengen nach und ist somit für die Behandlung einer reaktiven bzw. refraktären Parodontitis von sehr großer Bedeutung.

Profil: Parodontitis-Markerkeimnachweis/PCR

\section{Quecksilberbelastung}

\section{Kaugummi-Test}

Zur diagnostischen Beurteilung einer amalgambedingten Quecksilberbelastung im Mundbereich wird Quecksilber im Speichel vor und nach zehnminütigem intensiven Kauen von zuckerfreiem Kaugummi bestimmt. Erhöhte Werte finden sich bei nicht optimaler Oberfläche von Amalgam-Füllungen oder auch bei Vorhandensein zahlreicher Füllungen.

Profil: • 2 x Quecksilber im Speichel

- Kaugummi-Test

\section{Quecksilber im Urin}

Im Morgenurin ist die Ausscheidung an Quecksilber messbar. Diese Methode dient zur Abschätzung des Risikos für eine chronische Belastung. Untersuchung: Quecksilber im Urin Material: Morgenurin

- Untersuchung: Quecksilber im Morgenurin

\section{Quecksilber im Haar}

Überblick der Langzeitbelastung des Organismus (1-6 Monate). Die Quecksilberkonzentration im Haar liefert einen Anhaltspunkt über die Belastung des Nervensystems. Profil: • Quecksilber im Haar Dieser Test forciert die Quecksilber- bzw. Metallausscheidung und gibt einen Anhaltspunkt für die Einlagerung dieser Stoffe in den Organen, speziell der Niere. Das Quecksilber bzw. die Metalle werden mit DMPS gebunden und mit dem Urin ausgeschieden. Vorwiegend geeignet zur Quecksilberausleitung nach Amalgamsanierung, um den Körper von Quecksilberrückständen zu befreien.

- Quecksilber im Urin nach DMPS

\section{Quecksilber, Zinn, Kupfer im Urin nach DMPS}

Zusätzlich zum Quecksilber werden Zinn und Kupfer bestimmt, die ebenfalls Bestandteil des Amalgams sind.

Profil: • Quecksilber, Zinn, Kupfer im Urin

\section{- Schilddrüsen-Tests}

Auf Patientenwunsch werden beim Hausarzt häufig Schilddrüsen-Tests gefordert, obwohl keine Erkrankung vorliegt und damit die Tests nicht im Rahmen der GKV abrechnungsfähig sind.

Schilddrüsentests: fT3, fT4, T3, T4, TSH

\section{„Fit in der Schwangerschaft"“}

In der Schwangerschaft ist der Bedarf des Körpers an Spurenelementen, Vitaminen und Mineralien stark erhöht. Gleichzeitig ergeben sich aufgrund der hormonellen Umstellung Risiken bezüglich einer erhöhten Thromboseneigung. Bekanntlich werden nicht alle Infektionsrisiken durch die Mutterschaftsvorsorgeuntersuchung abgedeckt. Es sollte aber bereits frühzeitig in der Schwangerschaft festgestellt werden, ob die werdende Mutter immun gegen die wichtigsten dieser Infektionen ist oder aber auf diese Risiken vermehrt achten sollte.

\section{Schwangerschafts-Screening}

Profil: Stoffwechsel/Ernährung/Spurenelemente: Selen/Zink/Ferritin/Ca/Mg; Vitalstoffe:

Vit B1/Vit B6/Vit B12/Folsäure/Glucose; Hormone: TSH; Gerinnung; (2-Glykoprotein-AK, APC-Resistenz 
Untersuchung: DOWN-Risiko: freies Östriol-, AFP-, hCG-pränatal Untersuchung: Toxoplasmose-Suchtest

Schwangerschafts-Screening/Infektion: CMV-IgG, Parvo B19 IgG, Toxo IgG, Chlamydia IgG, IgA, VZV-lgG, Anti-HCV

\section{Sexualhormone beim Mann}

Auf Patientenwunsch werden beim Hausarzt häufig Sexualhormone-Tests gefordert, obwohl keine Erkrankung vorliegt und damit die Tests nicht im Rahmen der GKV abrechnungsfähig sind.

Hormonprofil: LH, SHBG, Testosteron

\section{- Sportler-Übertraining}

Extreme sportliche Betätigung (Hochleistungssport) führt nicht selten zu einer Immunsuppression, die sich in einem Leistungsknick und häufigen Infekten manifestieren kann. Grund dafür ist eine Hemmung der natürlichen Killerzellfunktion, eine Prävalenz von Suppressor-zellen, Abfall der natürlichen Schleimhautimmunität mit vermindertem sekretorischem IgA und prolongierte metabolische Veränderungen, vor allem im Lactat- und Glutaminhaushalt.

Profil: Leuko, sLgA, Lymphozytendifferenzierung, Glutamin, CK MB, Myoglobin, Lactat, Carnitin

\section{- Stressimmunität}

Starke berufliche Belastung, Abgeschlagenheit, Leistungsknick, Stress können sekundäre Defekte der zellulären Immunabwehr induzieren. Als Folge treten rezidivierende oder auch chronische Infekte, Müdigkeit oder Antriebsschwäche auf. Das Profil „Stressimmunität“ umfasst das zelluläre Immunprofil und die Kontrolle der wichtigsten hormonellen Stressursache, der Hypophysen-Nebennierenrindenaktivität.

Profil: 4 x Cortisol im Speichel, 1 x DHEA im Speichel, Blutbild; Lymphozytendifferenzierung (LYMS)

\section{- Toxikologie/Umwelt}

\section{Drogenscreening Urin}

Profil: Kokain, Opiate, Barbiturate, Benzodiazepine, Amphetamine, Cannabinoide, Methadon, LSD

\section{Multielementanalyse Urin oder Haare}

Die Haarmineralanalyse ermöglicht einen Überblick über die Aufnahme verschiedener Metalle in den letzten Monaten. Man kann bereits länger zurückliegende Schadstoffbelastungen nachweisen, bzw. die langfristige Versorgung des Körpers mit wichtigen Mineralien und Spurenelementen untersuchen.

Profil: Kupfer, Zink, Blei, Cadmium, Gold, Quecksilber, Titan, Cobalt, Indium, Gallium, Platin, Silber, Zinn, Palladium, Molybdän

Toxikologisches Profil: Urin oder Haare

Profil: Quecksilber, Blei, Cadmium, Arsen, Thallium, Aluminium, Chrom

Multiple Chemical Sensitivity (MCS)

Multiple Chemikalienempfindlichkeiten können durch einen Overload an Schadstoffen mit resultierender Überlastung des körpereigenen Schadstoffeliminationssystems verursacht werden.

Basisprofil: Quecksilber im Morgenurin, PCB, Blei, Cadmium, Lindan, CKW, GST, Detox-Assay (Coffeinmetaboliten der Entgiftungsphase I und II)

\section{Raumluftanalyse}

Die Belastung durch Lösungsmittel steigt im Wohn- und Arbeitsbereich durch die vielfältige Anwendung leichtflüchtiger Lösungsmittel. Mit Hilfe des ORSA 5 Passivsammlers lassen sich diese Substanzen sammeln und leicht nachweisen.

\section{Hausstaubanalyse}

In Möbeln, Teppichen, Tapeten und Raumtextilien werden verschiedenste Pestizide zum Schutz gegen Insekten oder Schimmelpilzbefall eingesetzt. Die Hausstaubuntersuchung bietet einen schnellen Überblick über die Belastungssituation durch Holzschutzmittel, PAK's und Weichmacher.

Untersuchung: Hausstaub-Material: Staubsaugerbeutel 


\section{Schimmelpilzbelastung im Wohnraum}

Schimmelpilze sind allgegenwärtige Organismen. Wo sie geeignete Lebensbedingungen antreffen, kann es zu einer starken Vermehrung kommen. Vor allem in Räumen, in denen durch bauliche Defizite oder versteckte Wasserschäden ein Feuchtigkeitsproblem besteht, finden Schimmelpilze einen idealen Nährboden. Auch im Isolationsmaterial bzw. hinter Wand- und Fußbodenverkleidungen kann sich Schimmel ansiedeln und gesundheitliche Beeinträchtigungen hervorrufen, indem lebende Schimmelpilze leichtflüchtige "chemische Kampfstoffe“, sog. MVOCs, an ihre Umgebung abgeben. Diese Substanzen können bei chronischer Belastung zu immunologischen Beeinträchtigungen mit erhöhter Infektanfälligkeit, Kopfschmerzen, Müdigkeit und Konzentrationsstörungen führen. Aber auch allergische Reaktionen treten bei empfindlichen Personen gegenüber Schimmel auf. In diesem Zusammenhang sind auch schon abgestorbene Schimmelreste noch relevant.

Profil: LTT-Schimmelpilze (Typ IV), LTC4-Schimmelpilze, Schimmelpilze IgE/lgG, Pilzkultur

\section{Neurotoxizitätstest}

Auch Belastungen mit Chemikalien (z. B. Formaldehyd) oder Metalle (z. B. Quecksilber) unter den üblichen toxikologischen Grenzwerten können bei kontinuierlicher Exposition zu Zellschädigungen führen, abhängig von der individuellen Schadstofftoleranz. Die biochemischen Mechanismen zur Schadstoffelimination sind nicht in allen Körperzellen gleichmäßig ausgeprägt. Leberzellen haben in dieser Hinsicht eine sehr hohe Leistungsfähigkeit, eher gering ausgeprägt ist dieses System in Nervenzellen, weshalb hier am ehesten eine Schädigung durch toxische Einflüsse mit resultierender Nervengewebezerstörung zu erwarten ist. Dies kann einerseits zur Produktion von Stress-Proteinen (Hitzeschockproteinen) bei den betroffenen Zellen führen, andererseits aber auch zum Auftreten von immunologischen Strukturen (Antikörper) gegen die normalerweise nicht vorhandenen Nervengewebsabbau-produkte.

Profil: Nerven-Antikörper, Hitzeschockprotein-Blot

\section{Tumorvorsorgeuntersuchung Labor - siehe auch Seite 136 Tumormarker}

Tumoren produzieren Substanzen, die im normalen Organismus nicht oder nur in sehr geringer Konzentration vorkommen (Tumormarker). Sie sind unter Umständen schon im Blut nachweisbar, wenn mit anderen Nachweismethoden (Röntgen etc.) noch keine Anzeichen für einen Tumor festgestellt werden können.

Erhöhte Tumormarker allein beweisen nicht das Vorhandensein eines Tumors, aber sie können unter Umständen sehr frühzeitig auf einen Tumor hinweisen.

Man unterscheidet Tumormarker ohne Organspezifität (z. B. CEA) von solchen mit hoher Organspezifität (z. B. PSA, CA 15-3)

Die Bestimmung von Tumormarkern ersetzt nicht andere Tumorvorsorgeuntersuchungen (z. B. Mammographie), aber sie kann diese sinnvoll ergänzen.

\section{Vaterschaftsgutachten}

Abstrich aus der Wangenschleimhaut

\section{Vitamine und Mineralstoffe}

- Vitaminprofil: Folsäure, Vitamin B6, Biotin

- Mineralprofil: Magnesium, Selen, Zink, Jod

- Vitamin/Mineralprofil: Zink, Folsäure, Vitamin B6, Jod, Magnesium

Komplett-Check: Profil: Vitamin B6, Biotin, Folsäure, Zink, Jod, Selen, Magnesium Natürlich können nach Patientenwunsch auch weitere oder andere Vitamine und/oder Mineralstoffe bestimmt werden. 


\section{Neurologie}

Was bietet die Fachgruppe der niedergelassenen Neurologen, Nervenärzte, Psychiater und ärztlichen Psychotherapeuten als IGeL-Leistungen an?

Vorsorge-Untersuchungen

- Fachbezogene Gesundheitsuntersuchung auf Wunsch des Patienten („Facharzt-Check“)

- Doppler-Sonographie der hirnversorgenden Gefäße bei fehlenden anamnestischen oder klinischen Auffälligkeiten

- Braincheck zur Früherkennung bei Demenzen

Psychotherapeutische Angebote

- Psychotherapeutische Verfahren zur Selbsterfahrung ohne medizinische Indikation

- Stressbewältigung

- Selbstbehauptungstraining

- Entspannungsverfahren

Präventionsleistung

- Biofeedback-Behandlung

- Kunst- und Körpertherapien, auch als ergänzende Therapieverfahren

- Verhaltenstherapie bei Flugangst

Alternative Heilverfahren

- Akupunktur (z. B. zur Schmerzbehandlung)

Ärztliche Serviceleistungen

- Ärztliche Untersuchungen und Bescheinigungen außerhalb der kassenärztlichen Pflichten auf Wunsch des Patienten (z. B. Bescheinigung für den Besuch von Kindergarten, Schule oder Sportverein oder bei Reiserücktritt)

- Untersuchung zur Überprüfung des intellektuellen und psychosozialen Leistungsniveaus (z. B. Schullaufbahnberatung auf Wunsch der Eltern)

- Gruppenbehandlung bei Adipositas

- Raucherentwöhnung

- Durchführung psychometrischer Tests

Sonstige Wunschleistungen

- Medizinisch nicht indizierte Abklärungsdiagnostik im Rahmen der Beweissicherung nach Drittschädigung

Neuartige Untersuchungs- und Behandlungsverfahren

- Bright-light-Therapie der saisonalen Depression

- Apparative Schlafprofilanalyse zur Diagnostik von Schlafstörungen

Zahlreiche Neurologen besitzen den Facharzt für Neurologie und Psychiatrie oder den Titel. Nervenarzt und sind aus diesem Grunde auch im Fachgebiet der Psychiatrie tätig.

Doppler-Sonographie der hirnversorgenden Gefäße bei fehlenden anamnestischen oder klinischen Auffälligkeiten - diese Untersuchung gehört zum Fachgebiet der Neurologie und in wenigen Fällen auch zum Leistungsangebot von Internisten.

Im Bereich der GKV sind Doppler-Sonographie und Duplex-Sonographie in Fällen, in denen weder anamnestische noch klinische Symptome oder Beschwerden auf eine Dauerstenose hinweisen, nicht Bestandteil des Leistungsumfanges. Die auf Wunsch des Patienten als individuelle Gesundheitsleistung durchgeführte doppler-sonographische Untersuchung der hirnversorgenden Gefäße bei Patienten,

- bei denen sich Schlaganfälle in der Familienanamnese befinden,

- die schlaganfallähnliche Symptome in der Anamnese aufweisen,

- bei denen eine Karotisstenose sonographisch festgestellt wurde, hat im Rahmen der präventiven Medizin nur Vorteile. 
E. Individuelle Gesundheitsleistungen von A - Z

Doppler-sonographische Untersuchungen der hirnversorgenden Gefäßen

\begin{tabular}{|l|l|r|r|}
\hline GOÄ-Nr. & Kurzlegende & $\mathbf{1 f a c h} \boldsymbol{€}$ & $\begin{array}{r}{ }^{* 1,8 /} \\
\mathbf{2 , 3 f a c h} €\end{array}$ \\
\hline $\mathbf{3}$ & $\begin{array}{l}\text { Eingehende Beratung (mind. 10 Min.) - nicht neben Sonderleis- } \\
\text { tungen }\end{array}$ & 8,74 & $\mathbf{2 0 , 1 1}$ \\
\hline $\mathbf{5}$ & Symptombezogene Untersuchung & 4,66 & $\mathbf{1 0 , 7 3}$ \\
\hline $\mathbf{8 0 0}$ & Neurologische Untersuchung & 11,37 & $\mathbf{2 6 , 1 4}$ \\
\hline $\mathbf{6 4 5}{ }^{\boldsymbol{1}}$ & Doppler-Sonographie & 37,89 & $\mathbf{6 8 , 2 0}$ \\
\hline $\mathbf{6 4 9}$ & Transkranielle Doppler-Sonographie & 37,89 & $\mathbf{6 8 , 2 0}$ \\
\hline
\end{tabular}

\section{Brain-Check}

\begin{tabular}{|l|l|r|r|}
\hline GOÄ Nr. & Kurzlegende & $\mathbf{1 f a c h} \boldsymbol{\mathbf { 2 , 3 f a c h }} \mathbf{€}$ \\
\hline $\mathbf{3}$ & $\begin{array}{l}\text { Eingehende Beratung (mind. 10 Min.) - nicht neben Sonderleis- } \\
\text { tungen }\end{array}$ & $\mathbf{8 , 7 4}$ & $\mathbf{2 0 , 1 1}$ \\
\hline $\mathbf{1}$ & Beratung & 4,66 & 10,72 \\
\hline $\mathbf{7}$ & Untersuchung eines Organsystems & 9,33 & 21,46 \\
\hline $\mathbf{8 0 0}$ & Neurologische Untersuchung & 11,37 & 26,15 \\
\hline $\mathbf{8 0 1}$ & Psychiatrische Untersuchung & $\mathbf{1 4 , 5 7}$ & $\mathbf{3 3 , 5 1}$ \\
\hline $\mathbf{8 5 7}$ & Orientierende Testuntersuchung & $\mathbf{6 , 7 6}$ & $\mathbf{1 5 , 5 5}$ \\
\hline
\end{tabular}

Zahlreiche Neurologen wenden im Rahmen der Schmerztherapie auch Akupunktur an. Hinweise dazu finden Sie im Kapitel Alternative Verfahren auf Seite $244 \mathrm{ff}$.

Labor-Untersuchung zur Feststellung des Herzinfarkt- und Apoplex-Risikos

\begin{tabular}{|c|c|c|c|}
\hline GOÄ-Nr. & Kurzlegende & 1 fach $€$ & $\begin{array}{r}{ }^{*} 1,8 / \\
2,3 f a c h \\
\end{array}$ \\
\hline 1 & Beratung, auch tel. & 4,66 & 10,72 \\
\hline 3 & Eingehende Beratung, auch tel. & 8,74 & 20,11 \\
\hline 5 & Symptombezogene Untersuchung & 4,66 & 10,73 \\
\hline 7 & $\begin{array}{l}\text { Untersuchung mind. eines Organssystems: Bauchorgane-- } \\
\text { Brustorgane - weibl. Genitaltrakt - Haut - Stütz- u. Bewegungs- } \\
\text { organe }\end{array}$ & 9,33 & 21,45 \\
\hline $250^{*}$ & Blutentnahme aus Vene & 2,33 & 4,20 \\
\hline GOÄ Nr. & Kurzlegende & 1fach $€$ & 1,15 fach $€$ \\
\hline 3562. $\mathrm{H} 1^{*}$ & Gesamt-Cholesterin & 2,33 & 2,68 \\
\hline 3563. $\mathrm{H} 1^{*}$ & HDL-Cholesterin & 2,33 & 2,68 \\
\hline $3564 . \mathrm{H} 1^{*}$ & LDL-Cholesterin & 2,33 & 2,68 \\
\hline $3565 . \mathrm{H} 1^{*}$ & Triglyceride & 2,33 & 2,68 \\
\hline $3560^{*}$ & Glukose & 2,33 & 2,68 \\
\hline $\begin{array}{l}4084^{*} \\
\text { analog }\end{array}$ & \begin{tabular}{|l|l|} 
Homocystein - analoger Ansatz - GOÄ § 6 (2) \\
GOÄ Kurztext Nr. 4084: Gesamt Östrogene im Urin, photometrisch \\
\end{tabular} & 32,22 & 38,21 \\
\hline $3933^{*}$ & Fibrinogen & 3,50 & 4,02 \\
\hline $3730^{*}$ & Lipoprotein (a) & 17,49 & 20,11 \\
\hline $3741^{*}$ & CRP (Ligandenassay) & 11,66 & 13,41 \\
\hline
\end{tabular}

Weiterführende Untersuchungen zu Herzinfarkt- und Schlaganfall-Risiko

- Koronararteriographie, nicht-invasive, z. B. mit Elektronenstrahl-Tomographie (EBCT) oder mit Mehrschicht-Computer-Tomographie, auch mit Bestimmung von Koronar-kalk

- Schlaganfall-Vorsorge: Doppler-Sonographie der hirnversorgenden Gefäße bei fehlenden anamnestischen oder klinischen Auffälligkeiten („Stroke-Check“) 


\section{Orthopädie}

Was bietet die Fachgruppe als IGeL-Leistungen an?

Akupunktur

- Schulterschmerz (Periarthropathia humeroscapularis)

- Tennisarm (Epicondylopathia humeri radialis)

- Kniebeschwerden

- Rückenschmerzen, Ischias

- Migräne, Spannungskopfschmerz

Biomagnetfeldtherapie

- bei Arthrose z. B. auch Fingerpolyarthrosen

- Schulterschmerz

- Rückenschmerz

\section{Ernährungsberatung}

Hyaluronsäureinjektionen

- zur Vorbeugung und zum Aufhalten von Kniegelenksarthrose

Hydro-Jet

- bei Rückenschmerzen

- Muskelverspannungen

Die Hydro-Jet Therapie kombiniert die Wirkungen einer Unterwasserdruckstrahlmassage und die einer Wärmetherapie. Dadurch sollen drei Effekte erreicht werden:

- Mechanisch-physikalische Behandlung zur Lockerung der Muskulatur

- Straffung des Gewebes

- Verbesserte Durchblutung

Der Patient liegt in Rücken-, Bauch- oder Seitenlage auf einer Latexmatte gewissermaßen auf dem Wasser, das sich um den Körper schmiegt. In kreisenden Bewegungen werden aus Düsen Wasserstrahlen gegen die Unterseite der Latex-Abdeckung und somit massierend gegen den Körper gerichtet.

\section{Knochendichtemessung}

- Osteo-Densitometrie

\section{Naturheilverfahren}

Leithoff und Sadler (Individuelle Gesundheitsleistungen in der Orthopädie, Thieme Verlag) halten für die orthopädische Praxis folgende Methoden/Verfahren der Naturheilkunde für besonders geeignet:

- Ausleitende Heilverfahren

- Neuraltherapie

- F.X. Mayr-Medizin, Heilfasten

- Ordnungstherapie, "lifestyle management“

- Phytotherapie, incl. Komplexhomöopathie

\section{Neuraltherapie}

- bei Kopfschmerz

- Vegetative Funktionsstörungen

- Erkrankungen des Bewegungsapparates

Physikalische Therapie

- Ultraschall

- Iontophorese

- Kryotherapie

Sonographie bei

- Weichteilerkrankungen

- Weichteilverletzungen

Sportmedizin

- Eignungsuntersuchungen für einzelne Sportarten

- Erstellung von Trainingsplänen 


\section{E. Individuelle Gesundheitsleistungen von A - Z}

Stoßwellentherapie (fokussierte) bei

- Fersensporn

- Schulterbeschwerden

- Tenniselbogen

\section{Hochton-Therapie}

Ziele einer Hochtontherapie sind z. B.

- Beschleunigung von Heilungsprozessen z. B. nach Knochenbrüchen, Quetschungen und Verstauchungen, Entzündungen (Tennisarm)

- Wiedererlangung der Muskelkraft nach Ruhigstellung

- Schnelle Wundheilung Ulcera (z. B. offene Beine)

- Schmerzlinderung bei Arthrosen, Gelenkbeschwerden.

In 70-80 Prozent der Fälle soll die Therapie erfolgreich sein. Ihre Wirkung ist mit wissenschaftlichen Studien bisher nicht belegt.

\begin{tabular}{|l|l|r|r|}
\hline GOÄ Nr. & Kurzlegende & $\mathbf{1 f a c h} \boldsymbol{€}$ & $\begin{array}{r}{ }^{*} \mathbf{1 , 8 /} \\
\mathbf{2 , 3 f a c h} \boldsymbol{€}\end{array}$ \\
\hline $\mathbf{3}$ & $\begin{array}{l}\text { Eingehende Beratung (mind. 10 Min.) - nicht neben Sonderleis- } \\
\text { tungen }\end{array}$ & 8,74 & $\mathbf{2 0 , 1 1}$ \\
\hline $\mathbf{5}$ & Symptombezogene Untersuchung & 4,66 & $\mathbf{1 0 , 7 3}$ \\
\hline $\begin{array}{l}\mathbf{5 5 4} \\
\text { analog }\end{array}$ & $\begin{array}{l}\text { Hochton-Therapie - analoger Ansatz - GOÄ § 6 (2) } \\
\text { GOÄ Kurztext Nr. 554: Hydroelektrisches Vollbad }\end{array}$ & 5,30 & $\mathbf{9 , 5 5}$ \\
\hline
\end{tabular}

\section{Injektionen im Bereich der Wirbelsäule}

\begin{tabular}{|c|c|c|c|}
\hline GOÄ Nr. & Kurzlegende & 1 fach $€$ & 2,3 fach $€$ \\
\hline 255 & $\begin{array}{l}\text { Injektion intraartikulär o. perineural (ggf. zusätzlich Lokalanästhe- } \\
\text { sie nach Nr. 490) - Nr. } 255 \text { ist nicht in der Schmerztherapie mit } \\
\text { Lokalanästhetika und/oder Analgetika berechenbar, sondern die } \\
\text { Nr. } 493 \text {. }\end{array}$ & 5,54 & 12,74 \\
\hline 256 & $\begin{array}{l}\text { Injektion in Periduralraum - bei Injektion von Lokalanästhetika } \\
\text { und/oder Analgetika sind Nrn. } 469 \text { bis } 475 \text { berechenbar }\end{array}$ & 10,78 & 24,80 \\
\hline 266 & $\begin{array}{l}\text { Intrakutane Reiztherapie (Quaddelung), je Sitzung (bei zeitauf- } \\
\text { wendiger Therapie ggf. höheren Steigerungssatz wählen) } \\
\text {-Nr. } 266 \text { ist im Rahmen der Schmerztherapie berechenbar }\end{array}$ & 3,50 & 8,04 \\
\hline 267 & $\begin{array}{l}\text { Medikamentöse Infiltrationsbehandlung einer Körperregion -auch } \\
\text { paravertebrale oder perineurale, je Sitzung -bei paravertebraler } \\
\text { Anästhesie Nr. } 476 \text { berechenbar. }\end{array}$ & 4,66 & 10,73 \\
\hline 268 & $\begin{array}{l}\text { Medikamentöse Infiltrationsbehandlung mehrerer Körperregionen } \\
\text { (auch eine Region beidseits), je Sitzung - } \\
\text { bei paravertebraler Anästhesie Nrn. 476, } 477 \text { berechenbar. }\end{array}$ & 7,58 & 17,43 \\
\hline
\end{tabular}

Die folgenden Leistungen finden Anwendung im Rahmen von Anästhesieleistungen, bei diagnostischen und/oder therapeutischen Eingriffen und im Rahmen der Schmerztherapie:

\begin{tabular}{|l|l|r|r|}
\hline GOÄ Nr. & Kurzlegende & $\mathbf{1 f a c h} €$ & $\mathbf{2 , 3 f a c h} €$ \\
\hline $\mathbf{4 6 9}$ & Kaudalanästhesie & 14,57 & $\mathbf{3 3 , 5 2}$ \\
\hline $\mathbf{4 7 0}$ & $\begin{array}{l}\text { Einleitung/Überwachung einzeitiger subarachnoidalen Spinalan- } \\
\text { ästhesie (Lumbalanästhesie) O. einzeitigen periduralen (epidura- } \\
\text { len) Anästhesie, bis zu 1 Stunde }\end{array}$ & 23,32 & $\mathbf{5 3 , 6 2}$ \\
\hline $\mathbf{4 7 6}$ & $\begin{array}{l}\text { Paravertebralanästhesie, bis zu 1 Stunde } \\
\text { Infiltrationsanästhesie kleiner Bezirke - in der Schmerztherapie } \\
\text { berechenbar }\end{array}$ & 22,15 & $\mathbf{5 0 , 9 4}$ \\
\hline $\mathbf{4 9 1}$ & $\begin{array}{l}\text { Infiltrationsanästhesie großer Bezirke in der Schmerztherapie be- } \\
\text { rechenbar }\end{array}$ & $\mathbf{8 , 0 5}$ \\
\hline
\end{tabular}




\begin{tabular}{|l|l|r|r|}
\hline GOÄ Nr. & Kurzlegende & $\mathbf{1 f a c h} €$ & $\mathbf{2 , 3 f a c h} €$ \\
\hline $\mathbf{4 9 3}$ & Leitungsanästhesie, perineural & 3,56 & $\mathbf{8 , 1 8}$ \\
\hline $\mathbf{4 9 4}$ & Leitungsanästhesie, endoneural & $\mathbf{7 , 0 5}$ & $\mathbf{1 6 , 2 2}$ \\
\hline $\mathbf{4 9 7 ^ { 1 } )}$ & $\begin{array}{l}\text { Blockade Truncus sympatikus (lumbaler Grenzstrang oder Gang- } \\
\text { lion stellatum) mittels Anästhetika - für Analgesie eines oder } \\
\text { mehrerer Spinalnerven analoger Ansatz }\end{array}$ & 12,82 & $\mathbf{2 9 , 4 9}$ \\
\hline $\mathbf{4 9 8 ^ { 1 } )}$ & $\begin{array}{l}\text { Blockade des Truncus sympatikus (thorakaler Grenzstrang oder } \\
\text { Plexus solaris) mittels Anästhetika }\end{array}$ & 17,49 & $\mathbf{4 0 , 2 2}$ \\
\hline $\mathbf{4 4 6}$ & $\begin{array}{l}\text { Zuschlag bei amb. Durchführung von Anästhesieleistungen z. B. } \\
\text { auch bei Schmerztherapie, die mit Punktzahl 200-399 Punkten } \\
\text { bewertet sind - nur 1facher Satz abrechenbar }\end{array}$ & $\mathbf{1 7 , 4 9}$ & - \\
\hline $\mathbf{4 4 7}$ & $\begin{array}{l}\text { Zuschlag bei amb. Durchführung von Anästhesieleistungen z. B. } \\
\text { auch bei Schmerztherapie, die mit Punktzahl 400 und mehr } \\
\text { Punkten bewertet sind - nur 1facher Satz abrechenbar }\end{array}$ & $\mathbf{3 7 , 8 9}$ & - \\
\hline
\end{tabular}

1) Die Leistungen sind, wenn sie in den nach der Leistungslegende beschriebenen Bereichen erbracht wurden, auch nebeneinander berechnungsfähig.

\section{Magnetfeldtherapie}

Die Abrechnung ist nach einem Tipp von Dr. Kleinken (Leiter der PVS Consult Köln, einer ServiceEinrichtung der Privatärztlichen Verrechnungsstellen) in der Broschüre IGEL-Plus aus dem Ärzte Zeitung Verlag abhängig vom Gerätetyp, seinem Leistungsspektrum und damit auch den Anschaffungskosten. Bei kostengünstigen Geräten werden in der Regel die GOÄ Nrn. A5802 oder 269a angesetzt. Bei hochwertigeren Geräten die GOÄ Nr. A838. Die Autorinnen Hess und Klakow-Franck geben in ihrem IGeL-Kompendium für die Arztpraxis den analogen Ansatz der GOÄ Nr. 558* an.

\section{Abrechnung}

\begin{tabular}{|c|c|c|c|}
\hline GOÄ Nr. & Kurzlegende & 1fach $€$ & $\begin{array}{r}{ }^{*} 1,8 / \\
2,3 f a c h €\end{array}$ \\
\hline 3 & $\begin{array}{l}\text { Eingehende Beratung (mind. } 10 \text { Min.) - nicht neben Sonderleistun- } \\
\text { gen }\end{array}$ & 8,74 & 20,11 \\
\hline 1 & Beratung & 4,66 & 10,73 \\
\hline 5 & Symptombezogene Untersuchung & 4,66 & 10,73 \\
\hline $\begin{array}{l}5802^{*} \\
\text { analog }\end{array}$ & $\begin{array}{l}\text { Bestrahlung bis zu } 2 \text { Behandlungsfeldern - analog GOÄ } \\
\text { Nr. } 5802^{\star} \text { - entsprechend GOÄ } \S 6(2) \text { - es muss mind. die Original- } \\
\text { Kurzlegende folgen }\end{array}$ & 11,66 & 20,98 \\
\hline $\begin{array}{l}269 a \\
\text { analog }\end{array}$ & $\begin{array}{l}\text { Magnetfeldtherapie - analog GOÄ Nr. } 269 a \text { - entsprechend GOÄ } \\
\S 6(2) \text { - es muss mind. die Original-Kurzlegende folgen }\end{array}$ & 20,40 & 46,92 \\
\hline $\begin{array}{l}838 \\
\text { analog }\end{array}$ & $\begin{array}{l}\text { Magnetfeldtherapie - analog GOÄ Nr. } 838 \text { - entsprechend GOÄ } \\
\S 6(2) \text { - es muss mind. die Original-Kurzlegende folgen }\end{array}$ & 32,06 & 73,73 \\
\hline $\begin{array}{l}558^{*} \\
\text { analog }\end{array}$ & $\begin{array}{l}\text { Magnetfeldtherapie - analog GOÄ Nr. } 558^{*} \text { - entsprechend GOÄ } \\
\S 6(2) \text { - es muss mind. die Original-Kurzlegende folgen }\end{array}$ & 7,00 & 12,59 \\
\hline
\end{tabular}

Manuelle Therapie - Chirotherapie - Osteopathie

Die GOÄ Leistungen nach den Nrn. 505* - 510* aus dem Kapitel Physikalisch-medizinische Leistungen und hier speziell: Krankengymnastik und Übungsbehandlungen finden Einsatz bei zahlreichen Behandlungsverfahren z. B.

- Ayurvedische Bewegungstherapie

- Chirotherapie

- Kraniosacrale Therapie

- Manuelle Therapie

- Myo-, neuro-, arthrotherapeutische Behandlungen

Manuelle Therapie - Chirotherapie

Indikationen und Kontraindikationen (Quelle: REHA Reinfelden, Schweiz http://www.reha-rheinfelden.ch/therapieangebot/chiropraktik.html) 
Eine geeignete Untersuchungstechnik (Manuelle Diagnostik, Chirodiagnostik) kann die jeweiligen Funktionsstörungen näher analysieren und geht der Indikationsstellung für eine Therapie voraus. Notwendig ist eine spezielle Ausbildung in Manueller Medizin, die sowohl Techniken der Diagnostik und Therapie beinhaltet.

Indikationen zur Chirotherapie (Manuellen Therapie) sind im wesentlichen:

- Bewegungsstörungen und Schmerzen der Wirbelsäule

- Kopf- und Nackenschmerzen

- Rückenschmerzen

- Schiefhals

- Schulter-Arm-Syndrome

- Schulter-, Hüft-, Knieschmerzen

- Tennis- und Golferellenbogen

- Hand- und Fußgelenkschmerzen

- Skoliosen

- ISG-Syndrome

Absolute Kontraindikationen sind:

- akut entzündliche Gelenkerkrankungen

- Bänderrisse und Gelenkinstabilitäten

- akute Frakturen oder Verrenkungen

- Infektionen

- Gefässerkrankungen

- akute Myelopathien

- akute Conus- oder Cauda-Syndrome

Relative Kontraindikationen sind:

- instabile Spondylolisthesen

- Osteoporose

- Knochentumoren

- Gelenkstörungen postoperativ

- generalisierte Gelenkhypermobilität

- Antikoagulation

\section{Abrechnung}

\begin{tabular}{|l|l|r|r|}
\hline GOÄ Nr. & Kurzlegende & 1fach $€$ & $\mathbf{2 , 3 f a c h ~} \mathbf{~}$ \\
\hline $\mathbf{3 3 0 5}$ & Chiropraktische Wirbelsäulenmobilisierung & 2,16 & 4,96 \\
\hline $\mathbf{3 3 0 6}$ & Chirotherapeutischer Eingriff an Wirbelsäule & 8,63 & 19,84 \\
\hline $\begin{array}{l}\mathbf{3 3 0 6} \\
\text { analog }\end{array}$ & $\begin{array}{l}\text { Chirotherapeutischer Eingriff an Extremitäten-Gelenken - ana- } \\
\text { log - entsprechend GOÄ § 6 (2) - pro Sitzung nur 1x }\end{array}$ & 8,63 & 19,84 \\
\hline
\end{tabular}

Fuchs und Brück halten eine Abrechnung der GOÄ Nrn. 3306 (Behandlung Wirbelsäule) + 3306 analog (Behandlung Extremitätengelenke) für möglich.

Im Zusammenhang mit Kraniosakraler Therapie, Ortho-Bionomie, Osteopathie und Kinesiologie etc. empfiehlt Weber noch nachfolgende Abrechnungsnummern - teilweise auch im analogen Ansatz.

\begin{tabular}{|l|l|r|r|}
\hline GOÄ Nr. & Kurzlegende & $\mathbf{1 f a c h} €$ & $\mathbf{2 , 3 f a c h} €$ \\
\hline $\mathbf{8 0 0}$ & Neurolog. Untersuchung & 11,38 & $\mathbf{2 6 , 1 4}$ \\
\hline $\mathbf{8 2 5}$ & Genaue Geruchs- und/oder Geschmacksprüfung ${ }^{11}$ & 4,84 & $\mathbf{1 1 , 1 3}$ \\
\hline $\mathbf{8 2 6}$ & $\begin{array}{l}\text { Gezielte neurologische Gleichgewichts- U. Koordininationsprü- } \\
\text { fung }\end{array}$ & 5,77 & $\mathbf{1 3 , 2 7}$ \\
\hline $\mathbf{8 3 0}$ & $\begin{array}{l}\text { Eingehende Prüfung auf Phasie, Apraxie, Alexie, Agraphie, Ag- } \\
\text { nosie und Körperschemastörungen }{ }^{11}\end{array}$ & 4,66 & $\mathbf{1 0 , 7 2}$ \\
\hline $\mathbf{8 3 5}$ & $\begin{array}{l}\text { Fremdanamnese bei psychisch Kranken o. bei verhaltensgestör- } \\
\text { tem Kind }\end{array}$ & 3,73 & $\mathbf{8 , 5 8}$ \\
\hline
\end{tabular}

1) Nrn. 825,826 und 830 nicht neben Nr. 800 


\section{Abrechnung}

Vor bestimmten Verfahren z. B. Maitland-Techniken, mit Kraniosakraler Therapie, Ortho-Bionomie, Osteopathie und Kinesiologie etc. empfiehlt Weber zusätzlich ggf. noch nachfolgende Abrechnungsnummern - teilweise auch im analogen Ansatz.

\begin{tabular}{|l|l|r|r|}
\hline GOÄ Nr. & Kurzlegende & 1fach $€$ & $\mathbf{2 , 3 f a c h} \mathbf{~}$ \\
\hline $\mathbf{8 3 8}$ & Elektromyographische Untersuchung & 32,06 & $\mathbf{7 3 , 7 3}$ \\
\hline $\mathbf{8 3 9}$ & $\begin{array}{l}\text { Elektromyographische Untersuchung mit Nervenleitgeschwindig- } \\
\text { keit }\end{array}$ & 40,80 & $\mathbf{9 3 , 8 4}$ \\
\hline $\mathbf{8 4 2}$ & Apparative isokinetische Muskelfunktionsdiagnostik & 29,14 & $\mathbf{6 7 , 0 3}$ \\
\hline
\end{tabular}

Progressive Muskelrelaxation

Die Progressive Muskelrelaxation ist ein Entspannungsverfahren. Ausgangspunkt war die Erkenntnis von Edmund Jacobson (USA), dass bei Anspannung und anschließender Entspannung eines Muskels der Muskeltonus sinkt.

Die Voraussetzung ist, dass der Übende den Anspannungs- und Entspannungszyklus erlernt:

- Dabei konzentriert sich der Patient auf eine bestimmte Muskelgruppe.

- Die Muskeln sollen angespannt werden und die Spannung sollte für 5 bis 7 Sekunden gehalten werden.

- Dann wird die Muskelgruppe wieder entspannt (ca. 20 bis 30 Sekunden).

- Der Klient konzentriert sich während der Lockerung auf seine Empfindungen.

- Nacheinander werden 16 Muskelgruppen des Körpers angespannt und entspannt. (Quelle: www.schmerz-beschwerden.de)

\section{Abrechnung}

\begin{tabular}{|l|l|r|r|}
\hline GOÄ Nr. & Kurzlegende & $\mathbf{1}$ fach $€$ & $\begin{array}{r}{ }^{*} \mathbf{1 , 8 /} \\
\mathbf{2 , 3 f a c h} \boldsymbol{€}\end{array}$ \\
\hline $\mathbf{5 0 5}^{*}$ & Atmungsbehandlung & 4,95 & $\mathbf{8 , 9 2}$ \\
\hline $\mathbf{5 0 7}^{*}$ & Krankengymnastische Teilbehandlung & 4,66 & $\mathbf{8 , 3 9}$ \\
\hline $\mathbf{5 1 0}^{*}$ & Ubungsbehandlung & 4,08 & $\mathbf{7 , 3 4}$ \\
\hline $\mathbf{5 1 4}^{*}$ & Extensionsbehandlung mit Wärmetherapie und Massage & 6,12 & $11, \mathbf{0 2}$ \\
\hline $\mathbf{5 1 6}^{* *}$ & Teilmassage & 3,79 & $\mathbf{6 , 8 2}$ \\
\hline $\mathbf{5 2 3}^{*}$ & Massage im extramuskulären Bereich & 3,79 & $\mathbf{6 , 8 2}$ \\
\hline $\mathbf{8 4 6}$ & Übende Verfahren Einzelbehandlung & 8,74 & $\mathbf{2 0 , 1 1}$ \\
\hline $\mathbf{8 4 7}$ & Übende Verfahren Gruppenbehandlung & 2,62 & $\mathbf{6 , 0 3}$ \\
\hline
\end{tabular}

Osteopathische Techniken

Das Osteopathie-Register informiert im Internet (www.osteopathie-register.de/osteopathie.shtml): In der Entwicklung der Osteopathie haben sich verschiedene Gebiete differenziert: Parietale Osteopathie

- Untersuchung und Behandlung der Gelenke in Funktion und Beweglichkeit in Abhängigkeit zu anderen Körpersystemen.

- Verwandte Techniken finden sich in der heutigen Zeit in der Manuellen Therapie und in der Chirotherapie.

Myofasciale Osteopathie

- Untersuchung und Behandlung von Muskel-, Bindegewebs- und Fascienschichten.

Viscerale Osteopathie

- Normalisierung der Organbeweglichkeit.

- Unterbrechung von negativen neurovegetativen Afferenzen.

- Wiederherstellung des internen Organmilieus. Kraniosakrale Osteopathie

- Normalisierung der Mobilität der Schädelknochen.

- Verbesserung der Liquorzirkulation.

- Entspannung der Meningealmembranen.

- Neurovegetative Integration. 


\section{E. Individuelle Gesundheitsleistungen von A - Z}

Die Dt. Akademie für Osteopathische Medizin e.V. - kurz DAOM (www.daom.de) - ist die Vereinigung von Ärzten u. Physiotherapeuten, die eine seit 1990 begonnene postgraduierte Ausbildung in osteopathischen Verfahren und osteopathischer Medizin leitet und anbietet.

Das Lehrkonzept der Deutschen Akademie für Osteopathische Medizin e.V. beinhaltet alle osteopathischen Behandlungsverfahren:

- Muskelenergietechniken

- Kraniosakrale Techniken

- Myofasziale Releasetechniken

- Viszerale Techniken

- Viszeroparietal-funktionelle Techniken

- CounterStrain-Techniken

- Lymphdrainage-Therapie

- Mobilisation und Manipulationan Wirbelsäule und Extremitäten.

Abrechnung häufig angesetzten Leistungen:

\begin{tabular}{|c|c|c|c|}
\hline GOÄ Nr. & Kurzlegende & 1 fach $€$ & 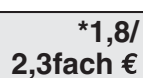 \\
\hline 800 & Eingehende neurologische Untersuchung & 11,37 & 26,14 \\
\hline 3305 & Chiropraktische Wirbelsäulenmobilisierung & 2,16 & 4,96 \\
\hline 3306 & Chirotherapeutischer Eingriff an der Wirbelsäule & 8,63 & 19,84 \\
\hline $505^{*}$ & Atmungsbehandlung & 4,95 & 8,92 \\
\hline $507^{*}$ & Krankengymnastische Teilbehandlung & 4,66 & 8,39 \\
\hline $520^{*}$ & Teilmassage (Massage einzelner Körperteile) & 2,62 & 4,72 \\
\hline $521^{*}$ & Großmassage & 3,79 & 6,82 \\
\hline $523^{*}$ & Massage im extramuskulären Bereich & 3,79 & 6,82 \\
\hline $\begin{array}{l}725 \\
\text { analog }\end{array}$ & $\begin{array}{l}\text { Entwicklungs- und Ubungsbehandlung von Ausfallerscheinungen } \\
\text { d. ZNS - analog GOÄ Nr. } 725 \text { - entsprechend GOÄ § } 6 \text { (2) - es } \\
\text { muss mind. die Original-Kurzlegende folgen }\end{array}$ & 17,49 & 31,48 \\
\hline
\end{tabular}

Kraniosakrale Therapie

\section{Abrechnung}

\begin{tabular}{|c|c|c|c|}
\hline GOÄ Nr. & Kurzlegende & 1 fach $€$ & $\begin{array}{r}{ }^{*} 1,8 / \\
2,3 \text { fach } € \\
\end{array}$ \\
\hline 269 & Akupunktur & 11,66 & 26,81 \\
\hline $523^{*}$ & Massage & 3,79 & 6,82 \\
\hline $\begin{array}{l}719 \\
\text { analog }\end{array}$ & $\begin{array}{l}\text { Funktioneller Entwicklungstherapie bei Ausfallerscheinungen in } \\
\text { Motorik, Sprachbereich und/oder Sozialverhalten, Einzelbehand- } \\
\text { lung, mind. } 45 \text { Min. - analog GOÄ Nr. } 719 \text { - entsprechend GOÄ } \\
\S 6 \text { (2) - es muss mind. die Original-Kurzlegende folgen }\end{array}$ & 14,63 & 33,65 \\
\hline $\begin{array}{c}725 \\
\text { analog }\end{array}$ & $\begin{array}{l}\text { Entwicklungs- und Ubungsbehandlung von Ausfallerscheinungen } \\
\text { d. ZNS - analog GOÄ Nr. } 725 \text { - entsprechend GOÄ § } 6 \text { (2) - es } \\
\text { muss mind. die Original-Kurzlegende folgen }\end{array}$ & 17,49 & 31,48 \\
\hline 845 & 1) Hypnose/Einzelbehandlung & 8,74 & 20,11 \\
\hline
\end{tabular}

1) Die Deutsche Gesellschaft für Manuelle Medizin hält im Gegensatz zu Weber, der die Leistung nach GOÄ Nr. 845 mitempfiehlt, diese Leistung für sehr bedenklich im Rahmen der Kraniosakralen Therapie

\section{Ortho-Bionomie}

Die Ortho-Bionomie ist nach Angaben der Anwender eine sanfte, manuelle Behandlungsmethode, um Schmerzen, Blockierungen im Bewegungsmuster, Fehlstellungen, Verspannungen, Gelenkbeschwerden- und Entzündungen zu behandeln.

Zur Abrechnung werden Leistungsziffern der Osteopathie angesetzt. Bei erforderlicher psychosomatischer Therapie und der Behandlung von psychischen Erkrankungen als Folge von Traumata finden zusätzlich die folgenden Leistungen Anwendung. 
Abrechnung der Ortho-Bionomie bei erforderlicher psychosomatischer Therapie und der Behandlung von psychischen Erkrankungen:

\begin{tabular}{|c|c|c|c|}
\hline GOÄ Nr. & Kurzlegende & 1fach $€$ & $2,3 f a c h €$ \\
\hline $\begin{array}{l}719 \\
\text { analog }\end{array}$ & $\begin{array}{l}\text { Behandlung von Ausfallerscheinungen, Einzelbehandlung, mind. } \\
45 \text { Min. - analog GOÄ Nr. } 719 \text { - entspr. GOÄ } \S 6(2) \text { - es muss } \\
\text { mind. die Original-Kurzlegende folgen }\end{array}$ & 14,63 & 33,65 \\
\hline 845 & Hypnose Einzelbehandlung - induzierte Tiefenentspannung & 8,74 & 20,11 \\
\hline 846 & Ubende Verfahren Einzelbehandlung & 8,74 & 20,11 \\
\hline 847 & Ubende Verfahren Gruppenbehandlung & 2,62 & 6,03 \\
\hline 849 & Psychotherapeutische Behandlung & 13,41 & 30,83 \\
\hline 861 & Tiefenpsychologisch fundierte Psychotherapie, Einzelbehandlung & 40,22 & 92,50 \\
\hline 862 & $\begin{array}{l}\text { Tiefenpsychologisch fundierte Psychotherapie, Gruppenbehand- } \\
\text { lung }\end{array}$ & 20,11 & 46,25 \\
\hline 870 & Verhaltenstherapie, Einzelbehandlung - Dauer mind 50 Minuten & 43,72 & 100,55 \\
\hline
\end{tabular}

Trager-Arbeit/Tragerwork

Der Trager-Verein aus Österreich informiert unter www.trager.at/wasist.htm zur Methode:

Die von Dr. med Milton Trager entwickelte Körperarbeit will Körper und Geist wieder in Harmonie bringen und die Bewußtheit darüber wecken. Alle Bewegungen und Erfahrungen werden als angenehm und wohltuend erlebt. Durch sanftes Dehnen, rhythmisches Schwingen und durch leichtes Spiel mit den Körpergewichten verändert sich der Gewebezustand, nähert sich seinem Optimum und weckt so angenehme Empfindungen. In einer TRAGER-Sitzung kann man lernen, diese Bewegungen selbst zu machen, man kann sie aber auch, auf einem Massagetisch liegend, passiv empfangen.

Indikationen

- Prävention:

Haltungsschäden

Gelenkserkrankungen

Stress und seine Folgen

Vegetative Störungen
- Rehabilitation:

Wirbelsäulensyndrom

Sportverletzungen

Unfallfolgen

Operationen
- Begleitende Therapie:

Neuromuskuläre Erkrankungen Stresserkrankungen (z. B.

Schlafstörungen, Tinnitus etc.) Gerontologie

Psychotherapie

\section{Abrechnung}

\begin{tabular}{|l|l|r|r|}
\hline GOÄ Nr. & Kurzlegende & $\mathbf{1 f a c h} €$ & $\begin{array}{r}{ }^{*} \mathbf{1 , 8 /} \\
\mathbf{2 , 3 f a c h} €\end{array}$ \\
\hline $\mathbf{5 2 3}^{*}$ & Massage im extramuskulären Bereich & 3,79 & $\mathbf{6 , 8 2}$ \\
\hline $\begin{array}{l}\mathbf{7 1 9} \\
\text { analog }\end{array}$ & $\begin{array}{l}\text { Behandlung von Ausfallerscheinungen in Motorik, Sprachbereich } \\
\text { und/oder Sozialverhalten, Einzelbehandlung, mind. 45 Min. - } \\
\text { analog GOÄ Nr. 523 - entsprechend GOÄ § 6 (2) - es muss mind. } \\
\text { die Original-Kurzlegende folgen }\end{array}$ & 14,63 & $\mathbf{3 3 , 6 5}$ \\
\hline $\mathbf{8 4 5}$ & Hypnose Einzelbehandlung & 8,74 & $\mathbf{2 0 , 1 1}$ \\
\hline $\mathbf{8 4 6}$ & Übende Verfahren Einzelbehandlung & 8,74 & $\mathbf{2 0 , 1 1}$ \\
\hline $\mathbf{8 4 7}$ & Übende Verfahren Gruppenbehandlung & 2,62 & $\mathbf{6 , 0 3}$ \\
\hline $\mathbf{3 3 0 5}$ & Chiropraktische Wirbelsäulenmobilisierung & 2,16 & $\mathbf{4 , 9 6}$ \\
\hline
\end{tabular}

Rebalancing

Rebalancing arbeitet z. B. mit tiefer Bindegewebsmassage, Gelenklockerung, Atemarbeit, Meditation. Erreicht werden soll eine langanhaltende Entspannung des Körpers. 
E. Individuelle Gesundheitsleistungen von A - Z

Abrechnung

\begin{tabular}{|l|l|r|r|}
\hline GOÄ Nr. & Kurzlegende & $\mathbf{1 f a c h} \boldsymbol{€}$ & $\begin{array}{r}\text { *1,8/ } \\
\mathbf{2 , 3 f a c h} €\end{array}$ \\
\hline $\mathbf{5 0 6}^{*}$ & Krankengymnastische Ganzbehandlung & 6,99 & $\mathbf{1 2 , 5 9}$ \\
\hline $\mathbf{5 0 7}^{*}$ & Krankengymnastische Teilbehandlung & 4,66 & $\mathbf{8 , 3 9}$ \\
\hline $\mathbf{5 2 3}^{*}$ & Massage im extramuskulären Bereich & 3,79 & $\mathbf{6 , 8 2}$ \\
\hline $\begin{array}{l}\mathbf{7 1 9} \\
\text { analog }\end{array}$ & $\begin{array}{l}\text { Behandlung von Ausfallerscheinungen in Motorik, Sprachbereich } \\
\text { und/oder Sozialverhalten, Einzelbehandlung - analog GOÄ Nr. 719 } \\
\text { - entspr. GOÄ § 6 (2) - es muss mind. die Original-Kurzlegende folgen }\end{array}$ & 14,63 & $\mathbf{3 3 , 6 5}$ \\
\hline $\mathbf{8 4 9}$ & Psychotherapeutische Behandlung & 13,41 & $\mathbf{3 0 , 8 3}$ \\
\hline
\end{tabular}

Trainingstherapie*

Eingesetzt wird diese Methode bei verschiedenen orthopädischen Krankheitsbildern z. B. bei Arthrosen, Osteoporose, Wirbelsäulenerkrankungen und -verletzungen sowie in der Nachbehandlung von Operationen und bei rheumatischen Beschwerden.

\begin{tabular}{|c|c|c|c|}
\hline GOÄ Nr. & Kurzlegende & 1 fach $€$ & $\begin{array}{r}{ }^{*} 1,8 / \\
2,3 \mathrm{fach} €\end{array}$ \\
\hline 3 & $\begin{array}{l}\text { Eingehende Beratung (mind. } 10 \text { Min.) - nicht neben Sonderleis- } \\
\text { tungen }\end{array}$ & 8,74 & 20,11 \\
\hline $506^{*}$ & Krankengymnastische Übungen, Einzelbehandlung & 7,00 & 12,59 \\
\hline $\begin{array}{l}558^{*} \\
\text { analog }\end{array}$ & $\begin{array}{l}\text { Geräte-Sequenztraining für Trainingstherapie - analoger Ansatz } \\
\text { - GOÄ § } 6 \text { (2) } \\
\text { Empfehlung der BÄK - GOÄ Kurztext Nr. 558: Apparative isokinetische Muskel- } \\
\text { funktionstherapie, je Sitzung }\end{array}$ & 7,00 & 12,59 \\
\hline $\begin{array}{l}842 \\
\text { analog }\end{array}$ & $\begin{array}{l}\text { Eingangsuntersuchung zur med. Trainingstherapie - analog - } \\
\text { GOÄ } \S 6(2) \\
\text { GOÄ Kurztext Nr. 842: Apparative isokinetische Muskelfunktionsdiagnostik }\end{array}$ & 29,14 & 67,03 \\
\hline $\begin{array}{l}846 \\
\text { analog }\end{array}$ & $\begin{array}{l}\text { Med. Trainingstherapie m. Sequenztraining, einschl. progressiv- } \\
\text { dynamischem Muskeltraining mit spez. Therapiemaschinen - } \\
\text { analog - GOÄ § } 6 \text { (2) } \\
\text { GOÄ Kurztext Nr. 846: Übende Verfahren }\end{array}$ & 8,74 & 20,11 \\
\hline
\end{tabular}

* diese Aufstellung entspricht den Empfehlungen der BÄK, s. Deutsches Ärzteblatt Jg. 99, Heft 3, Seite A144-145, 2002 - s. auch IGEL-Kompendium für die Arztpraxis von Hess und Klakow-Franck

\section{Triggerpunkt-Therapie}

Die sogenannte Triggerpunkt Therapie ist ein neues Verfahren der Schmerz- und der Bewegungsapparatmedizin. Die Internetseite aus der Schweiz www.triggerpunkt-therapie.ch/index.php?kat=home\&link=2\&language=de informiert:

Triggerpunkte sind die Folge von kleinen Verletzungen in der Muskulatur, wie sie zum Beispiel beim Muskelkater oder bei leichten Muskelzerrungen auftreten. In der Regel heilen diese Verletzungen wieder ab, denn die Muskulatur kann sich sehr gut erneuern. In gewissen Fällen aber, zum Beispiel wenn bestimmte Muskelfasern immer wieder die gleichen Bewegungen ausführen müssen, entsteht eine Art „anhaltender lokaler Muskelkater" und die betroffenen Muskelfasern entzünden sich chronisch. Das Gleiche kann auch nach einer brüsken Bewegung im Sport oder im Alltag oder nach einer Verletzung geschehen. Es bildet sich eine sogenannte „chronische Zerrung", die von alleine nicht mehr abheilen kann weil sich ein Teufelskreis gebildet hat.

\begin{tabular}{|l|l|r|r|}
\hline GOÄ Nr. & Kurzlegende & $\mathbf{1 f a c h} €$ & $\begin{array}{r}\text { *1,8/ } \\
\mathbf{2 , 3 f a c h ~} €\end{array}$ \\
\hline $\mathbf{3}$ & $\begin{array}{l}\text { Eingehende Beratung (mind. 10 Min.) - nicht neben Sonderleis- } \\
\text { tungen }\end{array}$ & 8,74 & $\mathbf{2 0 , 1 1}$ \\
\hline $\begin{array}{l}\mathbf{3 0 2} \\
\text { analog }\end{array}$ & Punktion Schulter- od. Hüftgelenk - analoger Ansatz - GOÄ § 6 (2) & 14,57 & $\mathbf{3 3 , 5 2}$ \\
\hline
\end{tabular}


Ultraschall-Behandlung*

Eingesetzt wird diese Methode bei verschiedenen orthopädischen Krankheitsbildern z. B. bei Arthrosen, Osteoporose, Wirbelsäulenerkrankungen und -verletzungen sowie in der Nachbehandlung von Operationen und bei rheumatischen Beschwerden.

\begin{tabular}{|l|l|r|r|}
\hline GOÄ Nr. & Kurzlegende & $\mathbf{1 f a c h} \boldsymbol{€}$ & $\begin{array}{r}\text { *1,8/ } \\
\mathbf{2 , 3 f a c h ~} \mathbf{€}\end{array}$ \\
\hline $\mathbf{3}$ & $\begin{array}{l}\text { Eingehende Beratung (mind. 10 Min.) - nicht neben Sonderleis- } \\
\text { tungen }\end{array}$ & 8,74 & $\mathbf{2 0 , 1 1}$ \\
\hline $\mathbf{5 5 4 *}$ & $\begin{array}{l}\text { Behandlung m. niedrig dosiertem, gepulsten Ultraschall - ana- } \\
\text { log-GOÄ § 6 (2) } \\
\text { GOÄ Kurztext Nr. 554*: Hydroelektrisches Vollbad }\end{array}$ & 5,30 & $\mathbf{9 , 5 5}$ \\
\hline
\end{tabular}

* niedrig dosierter, gepulster Ultraschall

\section{Osteoporose-Vorsorge}

Osteoporose-Risikofaktoren z. B. nach Leithoff und Sadler

- Postmenopausale Frauen mit Risikofaktoren

- Hypermenorrhoe oder Amenorrhoe/Hypoganadismus

- Anorexia nervosa

- Osteopenie im Skelettröntgen

- Alkoholabusus

- Nikotinabusus

- Immobilisation (mehr als 4 Wochen)

- Malabsorptionssyndrom (z. B. postoperativ)

- Hyperkalziurie

- Rheumatoide Arthritis, M. Bechterew

- Chron. Medikation mit Kortison

- Chron. Niereninsuffizienz

- Dialysepatienten

- Hyperparathyreodismus

- Hyperthyreose

- Suppressionstherapie an der Schilddrüse (seit mehr als 10 Jahren)

- Leberzirrhose

Liegen ein oder gar mehrere Risikofakoren vor, ist eine Osteodensitometrie zur frühzeitigen Erfassung einer pathologischen Demineralisierung indiziert.

Osteodensitometrie (Knochendichtemessung)

\begin{tabular}{|c|c|c|c|}
\hline GOÄ Nr. & Kurzlegende & 1 fach $€$ & ${ }^{*} 1,8$ fach $€$ \\
\hline $5380^{*}$ & $\begin{array}{l}\text { Osteodensitometrie von Skelettteilen mit quant. CT oder digita- } \\
\text { ler Röntgentechnik }\end{array}$ & 17,49 & 31,48 \\
\hline $5377^{*}$ & $\begin{array}{l}\text { Zuschlag für computergesteuerte Analyse, einschl. 3D-Rekon- } \\
\text { struktion }\end{array}$ & 46,63 & - \\
\hline $5475^{\star}$ & $\begin{array}{l}\text { Osteodensitometrie von Skelettteilen mittels Dual-Photonen-Ab- } \\
\text { sorptionstechnik }\end{array}$ & 17,49 & 31,48 \\
\hline
\end{tabular}

Abrechnungshinweis: Der Zuschlag nach Nr. 5377* ist nur mit 1fachem Gebührensatz abrechenbar.

Laboruntersuchungen bei VD Osteoporose

\begin{tabular}{|c|c|c|c|c|}
\hline GOÄ Nr. & Kurzlegende & Labor & 1 fach $€$ & 1,15 fach $€$ \\
\hline $3555^{\star}$ & Calcium & & 2,33 & 2,68 \\
\hline $3580 \mathrm{H}^{*}$ & Anorganischer Phosphor & & 2,33 & 2,68 \\
\hline $3587 \mathrm{H}^{*}$ & Alkalische Phophatase & & 2,33 & 2,68 \\
\hline $3585 \mathrm{H}^{*}$ * & Kreatinin & & 2,33 & 2,68 \\
\hline
\end{tabular}


Weitere Untersuchungen:

- Pyridinolin-Crosslinks und Ostase erfassen den Knochenabbau

- Vitamin D (25-OH) erfasst die Vitamin-D-Versorgung

Stoßwellentherapie bei orthopädischen Krankheitsbildern

Extrakorporale Stoßwellentherapie zur Behandlung von schmerzhaften Sehnenansätzen, Fersensporn, Kalkschulter, Tennisellenbogen.

\begin{tabular}{|l|l|r|r|}
\hline GOÄ Nr. & Kurzlegende & $\mathbf{1 f a c h} \boldsymbol{\mathbf { 2 , 3 f a c h }} \mathbf{}$ \\
\hline $\begin{array}{l}\mathbf{1 8 0 0} \\
\text { analog }\end{array}$ & $\begin{array}{l}\text { Extrakorporale Stoßwellentherapie (ESWT) bei orthopädischen, } \\
\text { chirurgischen oder schmerztherapeutischen Indikationen - ana- } \\
\text { log Nr. 1800 je Sitzung - Empfehlung der BÄK - GOÄ § 6 (2) } \\
\text { GOÄ Kurztext Nr. 1800: Zertrümmerung und Entfernung von Blasensteinen }\end{array}$ & 86,27 & $\mathbf{1 9 8 , 4 1}$ \\
\hline $\begin{array}{l}\mathbf{3 0 2} \\
\text { analog }\end{array}$ & $\begin{array}{l}\text { Radiale Stoßwellentherapie bei orthopädischen, chirurgischen } \\
\text { oder schmerztherapeutischen Indikationen - analog - Empfeh- } \\
\text { lung der BÄK - GOÄ § 6 (2) } \\
\text { GOÄ Kurztext Nr. 302: Punktion eines Schulter- oder Hüftgelenks }\end{array}$ & 14,57 & $\mathbf{3 3 , 5 2}$ \\
\hline $\mathbf{3 4}$ & Erörterung mind. 20 Min. & 17,49 & $\mathbf{4 0 , 2 2}$ \\
\hline
\end{tabular}

\section{Abrechnungshinweis:}

Auch wenn mehrere verschiedene Körperareale in einer Sitzung behandelt werden, ist nach dem GOÄ-Kommentar von Brück die Leistung nur einmal berechnungsfähig. Der Ausschuss Gebührenordnung der BÄK geht pro Behandlungsfall von durchschnittlich 2 bis 3 - maximal 4 -Behandlungen aus.

Die Indikation zur Stoßwellentherapie bei „Pseudarthrose“ ist nach Brück selten und zur Behandlung sind traumatologische Erfahrungen erforderlich. Wegen des Schwierigkeitsgrades und des hohen Zeitaufwandes bei der Behandlung der Pseudarthrose durch ESWT ist nach Brück ein Ansatz des 3,5fachen Steigerungsfaktors oder sogar eine Vereinbarung nach GOÄ § 2 (1) (Abweichende Gebührenhöhe) gut begründbar. 


\section{Pädiatrie}

Was bietet die Fachgruppe als IGeL-leistungen an?

Adoptionsgutachten/Entwicklungseinschätzungen

ADS-Diagnostik

Akupunktur

Allergietestungen ohne medizinische Indikation

Eigenblutbehandlung

Einschulungstest

Ernährungsberatung, individuelle

- z. B. zu Nahrungsergänzung mit Vitaminen, Spurenelementen und sekundären Pflanzenstoffen

Entwicklungstests

Farbsinnprüfung

Homöopathische Komplextherapien für Kinder

Impftiter-Bestimmungen

zusätzliche Impfungen außerhalb der Kassenleistungen z. B.

- Rotavirusschluckimpfung

- Meningokokken-Impfung

- Hepatitis A-Impfung Humanes

- Papilloma-Virus-Impfung

Kindergarteneingangsuntersuchung

Naturheilkunde

Psychotherapien

Schuleingangstests

Schullaufbahnberatung

Sportärztliche Untersuchungen

Stress-Bewältigungstraining (Einzel- und Gruppenbehandlung)

Umweltmedizin

- Umweltmedizinisch orientierende Beratung

- Umweltmedizinische Erstanamnese, Folgeanamnese

- z. B. Schwermetalle im Blut

spezielle Untersuchungen auf Wunsch der Eltern u. a.

- Labor-Untersuchungen

- Lungenfunktion

- Neurologische Untersuchung

- Ultraschall-Untersuchungen

zusätzliche Vorsorgeuntersuchungen

Die Zeitabstände zwischen Vorsorgeuntersuchungen zu Lasten der Krankenkassen sind nach Meinung der Pädiater zu lang und Entwicklungsprobleme würden möglicherweise zu spät erkannt. Deshalb bieten zahlreiche Pädiater zusätzliche Untersuchungen in kürzereren Zeitabständen an - und setzen diesen Untersuchungen zur Unterscheidung den Buchstaben „a“ hinzu z. B.

- U6a Vorsorgeuntersuchung mit Entwicklungs-Check zwischen 14. und 18. Lebensmonat

- U7a Vorsorgeuntersuchung mit 3 Jahren: Entwicklungs-Check, Hörprüfung, Sehtest und Urinuntersuchung

- 10a Vorsorgeuntersuchung im 8. Lebensjahr: Entwicklungs-Check, Hörprüfung, Sehtest und Überprüfung des Immunstatus

- 11a Vorsorgeuntersuchung im 10. Lebensjahr: Entwicklungs-Check, Hörprüfung, Sehtest und Überprüfung des Immunstatus

Häufige Wunschleistungen ohne Kostenerstattung durch die Krankenkasse und damit also IGEL-Leistungen im Bereich der Behandlung von Kindern und Jugendlichen sind z. B.

- zusätzliche jährliche Kinderfrüherkennungsuntersuchung (Intervallcheck) für Kinder bis zum vollendeten 7. Lebensjahr

- Kindervorsorgeuntersuchung mit 3 Jahren

- Tauglichkeitsuntersuchung, bei Kindern z. B. vor Kindergarten, Kurantritt, Ferienmaßnahme usw.

- Schuleignungstest-Untersuchung zur Überprüfung des intellektuellen und psychosozialen Leistungsniveaus (Schullaufbahnberatung)

- Vorsorgeuntersuchung in der 2. bzw. 4. Klasse 
- Test bei Aufmerksamkeits- und Konzentrationsstörung (ADS), (Intelligenztest, Konzentrationstest, Test auf Teilleistungsprobleme)

- Reisemedizinische Beratung für Kinder und Jugendliche

- Sportmedizinische Beratung für Kinder und Jugendliche

- Kurze Bescheinigung der Infektfreiheit ohne Untersuchung

\section{Prävention}

Dieses Kapitel beschäftigt sich mit der allgemeinen und individuellen Prävention mit zusätzlichen Untersuchungen auf Elternwunsch bei Kindern und Jugendlichen.

Im Rahmen der Leistungpflicht der gesetzlichen Krankenkassen (GKV) sind 10 Untersuchungen zur Früherkennung von Krankheiten bei einem Kinde festgelegt, an denen sich die PKV teilweise orientiert. Die Untersuchungen U1 bis U9 sind in einem Zeitraum vom Neugeborenen bis zum Alter von 60-64 Monaten auszuführen.

Die sog. U 10, die in der Legende der EBM-Nr. 01720 als „Jugendgesundheitsuntersuchung“ bezeichnet wird, ist den Richtlinien gemäß zwischen dem vollendeten 13. und vollendeten 14. Lebensjahr zu erbringen, allerdings mit einer Toleranzgrenze von 12 Monaten vor dem 13. Lebensjahr und 12 Monaten nach dem 14. Lebensjahr.

Während im EBM die Kinder-Richtlinien den Untersuchungsablauf regeln, findet sich in der GOÄ Nr. 26 in der Leistungslegende ein Hinweis auf den Untersuchungsinhalt, die Häufigkeit und die ausgeschlossenen Beratungs- und Untersuchungsleistungen. Die Früherkennungsuntersuchungen sind darauf ausgelegt, häufig vorkommende Erkrankungen festzustellen und dazu eine Diagnostik anzubieten, die auch von einer größeren Zahl von Ärzten durchgeführt werden kann.

Eltern GKV-versicherter Kinder haben häufig den Wunsch, vom 5. Lebensjahr (U bis U 9) bis zum 13. Lebensjahr ( $\cup 10)$ und dann wieder in der Zeit vom vollendeten 14. Lebensjahr bis zum 18. Lebensjahr zusätzliche Früherkennungsuntersuchungen durchführen zu lassen. Dieser Wunsch entspricht dem Bedürfnis von Jugendlichen und Eltern nach einer bestmöglichen Absicherung gegen Erkrankungen und Krankheiten.

Diese zusätzlichen Untersuchungen im Rahmen der IGEL-Liste - Kinder- und Jugendlichen-Intervall-Check genannt -, die zur frühzeitige Feststellung von möglichen Krankheiten oder Krankheitsneigungen führen können, haben später in der Gesetzlichen Krankenversicherung einen kostendämpfenden Effekt. Zum Kinder-Intervall-Check gehören auch die Überprüfung des Impfstatus des Patienten und evtl. Impfungen. 
Untersuchungen zur Früherkennung von Krankheiten bei einem Kind bzw. Jugendlichen nach der GKV und PKV

\begin{tabular}{|c|c|c|c|c|c|}
\hline $\begin{array}{l}\text { Untersu- } \\
\text { chungs- } \\
\text { stufe }\end{array}$ & $\begin{array}{l}\text { EBM } \\
2013\end{array}$ & $\begin{array}{l}\text { GOÄ } \\
\mathrm{Nr} \text {. }\end{array}$ & Zeitraum & Toleranzgrenze & Besondere Maßnahmen \\
\hline U1 & 01711 & & \multicolumn{3}{|c|}{ Unmittelbar nach der Geburt } \\
\hline U2 & 01712 & & 3.-10. Lebenstag & 3.-14. Lebenstag & TSH-Screenung \\
\hline U3 & 01713 & & $\begin{array}{l}\text { 4.-6. Lebenswo- } \\
\text { che }\end{array}$ & $\begin{array}{l}\text { 3.-8. Lebenswo- } \\
\text { che }\end{array}$ & $\begin{array}{l}\text { ggf. zusätzlich sonographi- } \\
\text { sche Screening-untersuchung } \\
\text { der Säuglingshüfte }\end{array}$ \\
\hline U4 & 01714 & & $\begin{array}{l}\text { 3.-4. Lebensmo- } \\
\text { nat }\end{array}$ & $\begin{array}{l}\text { 2.- }-4 \frac{1}{2} \text {. Lebens- } \\
\text { monat }\end{array}$ & \\
\hline U5 & 01715 & & $\begin{array}{l}\text { 6.-7. Lebensmo- } \\
\text { nat }\end{array}$ & $\begin{array}{l}\text { 5.-8. Lebensmo- } \\
\text { nat }\end{array}$ & \\
\hline U6 & 01716 & & $\begin{array}{l}\text { 10.-12. Lebens- } \\
\text { monat }\end{array}$ & $\begin{array}{l}\text { 9.-13. Lebensmo- } \\
\text { nat }\end{array}$ & \\
\hline U7 & 01717 & & $\begin{array}{l}\text { 21.-24. Lebens- } \\
\text { monat }\end{array}$ & $\begin{array}{l}\text { 20.-27. Lebens- } \\
\text { monat }\end{array}$ & \\
\hline U7A & - & & 3. Lebensjahr & - & $\begin{array}{l}\text { Allergien?, Verhaltensstörun- } \\
\text { gen?, Übergewicht?, Sprach- } \\
\text { störungen?, ZMK-Anomalien? }\end{array}$ \\
\hline U8 & 01718 & \multirow{2}{*}{26} & $\begin{array}{l}\text { 43.-48. Lebens- } \\
\text { monat }\end{array}$ & $\begin{array}{l}\text { 43.-50. Lebens- } \\
\text { monat }\end{array}$ & $\begin{array}{l}\text { Harnuntersuchung auf Ei- } \\
\text { weiss, Nitrit und ph }\end{array}$ \\
\hline U9 & 01719 & & $\begin{array}{l}\text { 60.-64. Lebens- } \\
\text { monat }\end{array}$ & $\begin{array}{l}\text { 58.-66. Lebens- } \\
\text { monat }\end{array}$ & $\begin{array}{l}\text { Harnuntersuchung auf Ei- } \\
\text { weiss, Nitrit und ph, Stereo- } \\
\text { test }\end{array}$ \\
\hline U10 & - & & 7.-8. Lebensjahr & - & $\begin{array}{l}\text { Entwicklungsstörungen (z. B. } \\
\text { Lesen-Rechtschreiben-Rech- } \\
\text { nen), Störungen der motor. } \\
\text { Entwicklung u.Verhaltensstö- } \\
\text { rungen (z. B.ADHS) }\end{array}$ \\
\hline U11 & - & & 9.-10. Lebensjahr & - & $\begin{array}{l}\text { Schulleistungs- u./o. Verhal- } \\
\text { tensstörungen?, Suchtmit- } \\
\text { teln?, Ernährungs-, Bewe- } \\
\text { gungs-, Stress-, Sucht- u. Me- } \\
\text { dienberatung }\end{array}$ \\
\hline $\mathrm{J1}^{*}$ & 01720 & & $\begin{array}{l}\text { 12.-14. Lebens- } \\
\text { jahr }\end{array}$ & - & \\
\hline J2 & - & & $\begin{array}{l}\text { 16.-18. Lebens- } \\
\text { jahr }\end{array}$ & - & $\begin{array}{l}\text { Pubertäts- und Sexualitätsstö- } \\
\text { rungen?, Haltungsstörungen, } \\
\text { Kropf?, Diabetes-Vorsorge, } \\
\text { Beratung bei der Berufswahl }\end{array}$ \\
\hline
\end{tabular}

Kinder- und Jugendlichen-Intervall-Checks

\begin{tabular}{|l|l|r|r|}
\hline GOÄ Nr. & Kurzlegende & $\mathbf{1 f a c h} €$ & $\begin{array}{r}{ }^{*} \mathbf{1 , 8 /} \\
\mathbf{2 , 3 f a c h} \boldsymbol{\text { f }}\end{array}$ \\
\hline $\mathbf{2 5}$ & Neugeborenen Erstuntersuchung - U1 & 11,66 & $\mathbf{2 6 , 8 1}$ \\
\hline $\begin{array}{l}\mathbf{2 6} \\
\text { analog }\end{array}$ & $\begin{array}{l}\text { Untersuchung zur Früherkennung von Krankheiten bei einem } \\
\text { Kind bis zum vollendeten 14. Lebensjahr - analog GOÄ § 6 (2) }\end{array}$ & 26,23 & $\mathbf{6 0 , 3 3}$ \\
\hline $\begin{array}{l}\mathbf{2 6} \\
\text { analog }\end{array}$ & $\begin{array}{l}\text { Untersuchung zur Früherkennung von Krankheiten bei einem } \\
\text { Kind bis zum vollendeten 18. Lebensjahr - analog GOÄ § 6 (2) }\end{array}$ & 26,23 & $\mathbf{6 0 , 3 3}$ \\
\hline
\end{tabular}


Abrechnungstipp: Bis zur Vollendung des 2. Lebensjahrs gelten keine Einschränkungen der Anzahl der durchgeführten Untersuchungen, $d$. h. wenn medizinisch erforderlich, können mehrere Untersuchungen nach GOÄ Nr. 26 abgerechnet werden. Ab dem 2. Lebensjahr bis zum vollendeten 14. Lebensjahr dürfen diese Untersuchungen allerdings nur einmal je Kalenderjahr berechnet werden.

Die Abrechnung zusätzlicher kurativer Leistungen bei einer „Untersuchung zur Früherkennung von Krankheiten bei einem Kind bis zum vollendeten 14. Lebensjahr" nach GOÄ Nr. 26 oder im Zeitraum vom 14.-18. Lebensjahr analog nach GOÄ Nr. 26 a - z. B. Laborparameter, Ultraschall, EKG etc. - ist jederzeit möglich, wenn im Rahmen der Leistung nach Nr. 26 Auffälligkeiten oder der Verdacht auf eine Erkrankung festgestellt werden

$\mathrm{Zu}$ allen Kinder- und Jugendlichen-Vorsorge-Untersuchungen und den entsprechenden Intervall-Checks gehören die Überprüfung des Impfstatus und evtl. Impfungen.

Vorsorgeuntersuchungen bei Kindern und Jugendlichen

U1-Neugeborenen Erstuntersuchung

\begin{tabular}{|l|l|r|r|}
\hline GOÄ Nr. & Kurzlegende & $\mathbf{1 f a c h} €$ & $\begin{array}{r}{ }^{*} 1,8 / \\
\mathbf{2 , 3 f a c h} €\end{array}$ \\
\hline 25 & U1-Neugeborenen-Erstuntersuchung & 11,66 & $\mathbf{2 6 , 8 1}$ \\
\hline
\end{tabular}

Besonderheiten dieser Vorsorgeuntersuchung: Apgar-Schema 1, 5, 10 Min. nach der Geburt. Vitamin-K-Prophylaxe bei U1, U2 und U3. Crede-Prophylaxe empfohlen.

U2-Vorsorgeuntersuchung 3.-10. Tag

\begin{tabular}{|c|c|c|c|}
\hline GOÄ Nr. & Kurzlegende & 1fach $€$ & $\begin{array}{r}{ }^{*} 1,8 / \\
2,3 f a c h \\
€\end{array}$ \\
\hline 26 & U2-Vorsorgeuntersuchung 3.- 10. Tag & 26,23 & 60,33 \\
\hline 413 & Sono der Hüftgelenke & 16,32 & 37,54 \\
\hline 50 & $\begin{array}{l}\text { Hausbesuch } \\
\text { ggf. zusätzlich: K2 (Zuschlag Kind) - bei Bedarf Zuschläge aus } \\
\text { dem Bereich E - H und Wegegeld }\end{array}$ & 18,65 & 42,90 \\
\hline $250^{*}$ & Blutabnahme für Guthrie Test & 2,33 & 4,20 \\
\hline & Labor & 1 fach $€$ & 1,15 fach $€$ \\
\hline $3758^{\star \star}$ & Phenylalanin (Guthrie Test) & 3,50 & **4,02 \\
\hline $4030^{\star *}$ & TSH-Bestimmung (nach GKV-Richtlinien am 5.Tag) & 14,57 & ** 16,76 \\
\hline
\end{tabular}

Besonderheiten dieser Vorsorgeuntersuchung: 2.Vitamin-K-Prophylaxe. Sono der Hüftgelenke. Screeninguntersuchungen auf Phenylketonurie, Galaktosämie, Mukoviszidose. Überprüfung des Impfstatus und ggf. erforderliche Impfungen.

U3-Vorsorgeuntersuchung 4.-6. Lebenswoche

\begin{tabular}{|l|l|r|r|}
\hline GOÄ Nr. & Kurzlegende & $\mathbf{1 f a c h} €$ & $\begin{array}{r}\text { *1,8/ } \\
\mathbf{2 , 3 f a c h} €\end{array}$ \\
\hline $\mathbf{2 6}$ & $\begin{array}{l}\text { U3-Vorsorgeuntersuchung 4-6. Lebenswoche } \\
\text { 3. Vitamin-K-Prophylaxe }\end{array}$ & 26,23 & $\mathbf{6 0 , 3 3}$ \\
\hline $\mathbf{4 1 3}$ & $\begin{array}{l}\text { Sono der Hüftgelenke ggf. Erstuntersuchung oder Kontrollunter- } \\
\text { suchung }\end{array}$ & 16,32 & $\mathbf{2 7 , 5 4}$ \\
\hline $\mathbf{7 1 5}$ & Prüfung kindliche Entwicklung & 12,82 & $\mathbf{2 9 , 4 9}$ \\
\hline $\mathbf{7 1 6}$ & $\begin{array}{l}\text { Prüfung funktionelle Entwicklung bei Säugling oder Kleinkind } \\
\text { nach standardisierten Methoden, je Untersuchungsgang }\end{array}$ & 4,02 & $\mathbf{9 , 2 5}$ \\
\hline $\mathbf{7 1 8}$ & $\begin{array}{l}\text { Höchstwert bei Untersuchungen nach Nr. 716 und 717; hier sind } \\
\text { die Untersuchungsarten anzugeben }\end{array}$ & 14,63 & $\mathbf{3 3 , 6 5}$ \\
\hline $\mathbf{1 4 0 9}$ & Otoakustische Emissionen & 23,32 & $\mathbf{5 3 , 6 2}$ \\
\hline
\end{tabular}


Wichtige Ausschlüsse: Nr. 715 nicht neben Nr. 26 und Nr. 1555 nicht neben den Nrn. 715, 717. Besonderheiten dieser Vorsorgeuntersuchung: 3.Vitamin-K-Prophylaxe. Erste Verhaltsmuster im Sozial- und Spielverhalten. Beurteilung der motorischen Entwicklung. Überprüfung des Impfstatus und ggf. erforderliche Impfungen.

U4-Vorsorgeuntersuchung 3.-4. Lebensmonat

\begin{tabular}{|l|l|r|r|}
\hline GOÄ Nr. & Kurzlegende & $\mathbf{1 f a c h} €$ & $\begin{array}{r}\text { *1,8/ } \\
\mathbf{2 , 3 f a c h} €\end{array}$ \\
\hline $\mathbf{2 6}$ & U4-Vorsorgeuntersuchung 3.-4. Lebensmonat & 26,23 & $\mathbf{6 0 , 3 3}$ \\
\hline $\mathbf{7 1 5}$ & Prüfung kindliche Entwicklung & 12,82 & $\mathbf{2 9 , 4 9}$ \\
\hline $\mathbf{7 1 6}$ & $\begin{array}{l}\text { Prüfung funktionelle Entwicklung bei Säugling oder Kleinkind } \\
\text { nach standardisierten Methoden, je Untersuchungsgang }\end{array}$ & 4,02 & $\mathbf{9 , 2 5}$ \\
\hline $\mathbf{7 1 8}$ & $\begin{array}{l}\text { Höchstwert bei Untersuchungen nach Nr. 716 und 717; hier sind } \\
\text { die Untersuchungsarten anzugeben }\end{array}$ & 14,63 & $\mathbf{3 3 , 6 5}$ \\
\hline $\mathbf{4 1 3}$ & Sono der Hüftgelenke ggf. Erstuntersuchg. od. Kontrolluntersuchg. & 16,32 & $\mathbf{2 7 , 5 4}$ \\
\hline $\mathbf{1 4 0 3}{ }^{*}$ & Tonschwellenaudiometrie & 9,21 & $\mathbf{1 6 , 5 8}$ \\
\hline $\mathbf{1 2 1 6}$ & Strabismusuntersuchung & 5,30 & $\mathbf{1 2 , 2 0}$ \\
\hline
\end{tabular}

Wichtige Ausschlüsse: Nr. 715 nicht neben Nr. 26, und Nr. 1555 nicht neben den Nrn. 715, 717. Besonderheiten dieser Vorsorgeuntersuchung:

Ausschluss zentraler Tonus- und Koordinationsstörungen. Seh- und Hörprüfungen. Überprüfung des Impfstatus und ggf. erforderliche Impfungen.

U5-Vorsorgeuntersuchung 6. - 7. Lebensmonat

\begin{tabular}{|c|c|c|c|}
\hline GOÄ Nr. & Kurzlegende & 1fach € &  \\
\hline 26 & U5-Vorsorgeuntersuchung 6.-7. Lebensmonat & 26,23 & 60,33 \\
\hline
\end{tabular}

Siehe auch Untersuchungen unter U4.

Besonderheiten dieser Vorsorgeuntersuchung: Ausschluss zerebraler Bewegungsstörungen. Beurteilung der geistigen Entwicklung. Überprüfung des Impfstatus und ggf. erforderliche Impfungen.

U6-Vorsorgeuntersuchung 10.-12. Lebensmonat

\begin{tabular}{|l|l|r|r|}
\hline GOÄ Nr. & Kurzlegende & 1fach $€$ & 2,3 fach $€$ \\
\hline 26 & U6-Vorsorgeuntersuchung 10.- 12. Lebensmonat & 26,23 & 60,33 \\
\hline
\end{tabular}

Siehe auch Untersuchungen unter U4 - Besonderheiten dieser Vorsorgeuntersuchung:

Neuroblastom-Früherkennung durch Screening auf Katecholamine im Urin („Windeltest") -(allerdings gibt es eine neue Studie, die zeigt, dass das Neuroblastomscreening nicht den Nutzen bringt, den man sich davon erhofft hatte: fortgeschrittenen Stadien früher erkennen zu können. Daher wird das Screening in der Studie nicht mehr empfohlen). - Sozialentwicklung (Fremdeln). • Untersuchung zur Früherkennung von Schwachsichtigkeit und Schielen -im Kleinkind- und Vorschulalter durch instrumentelle Untersuchung („Schiel-Vorsorge“). Ausschluss von Hörstörungen. Überprüfung des Impfstatus und ggf. erforderliche Impfungen.

U7-Vorsorgeuntersuchung 21.-24. Lebensmonat

U7A-Vorsorgeuntersuchung 3. Lebensjahr

\begin{tabular}{|l|l|r|r|}
\hline GOÄ Nr. & Kurzlegende & $\mathbf{1 f a c h ~} €$ & $\begin{array}{r}\text { *1,88 } \\
\mathbf{2 , 3 f a c h} €\end{array}$ \\
\hline $\mathbf{2 6}$ & U7-Vorsorgeuntersuchung 21.-24. Lebensmonat & 26,23 & $\mathbf{6 0 , 3 3}$ \\
\hline $\mathbf{1 4 0 0}$ & Hörprüfung & 4,43 & $\mathbf{1 0 . 1 9}$ \\
\hline $\mathbf{1 4 0 3}{ }^{\star}$ & Tonschwellenaudiometrie (Nr. 1403 nicht neben 1400) & 9,21 & $\mathbf{1 6 , 5 8}$ \\
\hline $\mathbf{2 6}$ & U7A-Vorsorgeuntersuchung 3. Lebensjahr & 26,23 & $\mathbf{6 0 , 3 3}$ \\
\hline
\end{tabular}


E. Individuelle Gesundheitsleistungen von A - Z

Siehe auch Untersuchungen unter U4 - Besonderheiten dieser Vorsorgeuntersuchung:

Beurteilung von Sinnes- und körperlicher Entwicklung. Überprüfung des Impfstatus und ggf. erforderliche Impfungen.

U8-Vorsorgeuntersuchung 43.-48. Lebensmonat

\begin{tabular}{|c|c|c|c|}
\hline GOÄ Nr. & Kurzlegende & 1 fach $€$ & $\begin{array}{r}{ }^{*} 1,8 / \\
2,3 \mathrm{fach} €\end{array}$ \\
\hline 26 & U8-Vorsorgeuntersuchung 43. - 48. Lebensmonat & 26,23 & 60,33 \\
\hline 1200 & Refraktion, subjektiv & 3,44 & 7,91 \\
\hline 1202 & Refraktion, objektiv & 4,31 & 9,92 \\
\hline 1216 & Strabismustest & 5,30 & 12,20 \\
\hline 1217 & Sehtest, apparativ & 14,11 & 32,44 \\
\hline 1228 & Farbsinnprüfung & 3,56 & 8,18 \\
\hline 1400 & Hörprüfung & 4,43 & 10.19 \\
\hline $1403^{*}$ & Hörprüfung & 9,21 & 16,58 \\
\hline $1406^{\star}$ & Kinderaudiometrie - bis zur Vollendung des 7. Lebensjahrs & 10,61 & 19,10 \\
\hline 1555 & Untersuchung der Sprache & 6,94 & 15,95 \\
\hline 384 & Tuberkulinstempeltest & 2,33 & 5,36 \\
\hline $857^{*}$ & Orientierende Testuntersuchung z. B. Haus-Baum-Mensch & 6,76 & 12,17 \\
\hline 410 & Sonographie Abdomen & 11,66 & 26,81 \\
\hline 420 & Sonographie Abdomen, je Organ, je Sitzung bis zu 3x & 4,66 & 10,73 \\
\hline $3511^{* \star}$ & Untersuchung des Urins & 2,91 & $\star \star 3,35$ \\
\hline
\end{tabular}

** Labor 1,15facher Satz

Wichtige Ausschlüsse: Nr. 715 nicht neben Nr. 26, und Nr. 1555 nicht neben den Nrn. 715, 717. Nr. 1400 nicht neben $1403^{*}$.

Abrechnungshinweis:

Nr. 1216 neben Nr. 1217 nur in besonderen Fällen. Nach Brück sind Indikationen dazu:

- Paresen

- Doppelbilder

- Schwankende Schielwinkel

- Kongenitales Schielsyndrom

- Kopf-Zwang-Haltungen

- Vor- und Nachuntersuchungen bei Schieloperationen

Besonderheiten dieser Vorsorgeuntersuchung: Erfassung von Verhaltensstörungen wie Einnässen, Einkoten, nicht altersgemäßer Sprache, Stereotypien. Überprüfung des Impfstatus und ggf. erforderliche Impfungen.

U9-Vorsorgeuntersuchung 60.-64. Lebensmonat

\begin{tabular}{|l|l|r|r|}
\hline GOÄ Nr. & Kurzlegende & 1fach $€$ & $\mathbf{2 , 3 f a c h} €$ \\
\hline 26 & U9-Vorsorgeuntersuchung 60.-64. Lebensmonat & 26,23 & $\mathbf{6 0 , 3 3}$ \\
\hline
\end{tabular}

Siehe Untersuchungen unter U8.

Besonderheiten dieser Vorsorgeuntersuchung: Seh- und Hörprüfung. Kontrolle der Motorik. Hand-Augen-Koordination. Sprachfähigkeit. Urinstatus. Überprüfung des Impfstatus und ggf. erforderliche Impfungen.

U10-Vorsorgeuntersuchung 7.-8. Lebensjahr

\begin{tabular}{|l|l|r|r|}
\hline GOÄ Nr. & Kurzlegende & 1fach $€$ & 2,3 fach $€$ \\
\hline 26 & U10-Vorsorgeuntersuchung 7. - 8. Lebensjahr & 26,23 & 60,33 \\
\hline
\end{tabular}


U11-Vorsorgeuntersuchung 9.-10. Lebensjahr

\begin{tabular}{|l|l|r|r|}
\hline GOÄ Nr. & Kurzlegende & 1fach $€$ & 2,3fach $€$ \\
\hline 26 & U11-Vorsorgeuntersuchung 9.- 10. Lebensjahr & 26,23 & 60,33 \\
\hline
\end{tabular}

Besonderheiten U10: Ausgedehnte Anamnese (chron. Erkrankungen, Behinderungen, Schulsituation, Familiensituation). Körperliche Untersuchung (Pubertätsentwicklung, Blutdruckmessung. Gesamtcholesterin-Bestimmung bei familiärer Belastung. - U11: Störungen der motor. Entwicklung u. Verhaltensstörungen (z. B. ADHS). Suchtmitteln? Ernährungs-, Bewegungs-, Stress-, Sucht- und Medienberatung. Überprüfung des Impfstatus und ggf. erforderliche Impfungen.

J1 (12.-14. Lebensjahr) und J2 (16.-18. Lebensjahr) Kinder-Früherkennungsuntersuchungen

\begin{tabular}{|l|l|r|r|}
\hline GOÄ Nr. & Kurzlegende & 1fach $€$ & $\mathbf{2 , 3 f a c h} €$ \\
\hline 26 & $\begin{array}{l}\text { J1 oder J2 Kinder-Früherkennungsuntersuchungen zwi- } \\
\text { schen dem 12. und 18. Lebensjahr }\end{array}$ & 26,23 & $\mathbf{6 0 , 3 3}$ \\
\hline
\end{tabular}

Besonderheiten dieser Vorsorgeuntersuchung: Überprüfung des Impfstatus und ggf. erforderliche Impfungen.

Hüftsonographie

\begin{tabular}{|l|l|r|r|}
\hline GOÄ Nr. & Kurzlegende & $\mathbf{1 f a c h ~} \mathbf{€}$ & $\begin{array}{r}\text { *1,8/ } \\
\mathbf{2 , 3 f a c h} \boldsymbol{€}\end{array}$ \\
\hline $\mathbf{1}$ & Beratung & 4,66 & 10,72 \\
\hline $\mathbf{5}$ & Symptombezogene Untersuchung & 4,66 & 10,72 \\
\hline $\mathbf{K 1}$ & $\begin{array}{l}\text { Zuschlag zu Untersuchungen nach Nrn. 5,6, 7 und 8 bei Kindern } \\
\text { bis zum vollendeten 4. Lebenjahr }\end{array}$ & $\mathbf{7 , 0 0}$ & \\
\hline $\mathbf{4 1 3}$ & $\begin{array}{l}\text { Sono der Hüftgelenke ggf. Erstuntersuchung oder Kontrollunter- } \\
\text { suchung bis vollendetes 2. Lebensjahr }\end{array}$ & 16,32 & 37,54 \\
\hline $\mathbf{1}$ & Beratung & 4,66 & $\mathbf{1 0 , 7 2}$ \\
\hline $\mathbf{5}$ & Symptombezogene Untersuchung & 4,66 & $\mathbf{1 0 , 7 2}$ \\
\hline $\mathbf{1 4 0 9}$ & Messung otoakustischer Emissionen & 23,31 & $\mathbf{5 3 , 6 2}$ \\
\hline $\mathbf{8 2 8}$ & $\begin{array}{l}\text { Messung visuell, akustisch oder sensosensorisch evozierter } \\
\text { Hirnpotentiale (VEP, AEP, SSP) }\end{array}$ & 35,26 & $\mathbf{8 1 , 1 1}$ \\
\hline
\end{tabular}

Neugeborenen Audio-Check

Die Abrechnung ist abhängig von der gewählten Methode

\begin{tabular}{|c|c|c|c|}
\hline GOÄ Nr. & Kurzlegende & 1 fach $€$ & ${ }^{* / 2,3 f a c h}$ \\
\hline 1 & Beratung, auch tel. & 4,66 & 10,72 \\
\hline 3 & Eingehende Beratung, auch tel. & 8,74 & 20,11 \\
\hline 5 & Symptombezogene Untersuchung & 4,66 & 10,73 \\
\hline 6 & HNO Untersuchung & 5,83 & 13,41 \\
\hline 828 & $\begin{array}{l}\text { Messung visuell, akustisch oder sensosensorisch evozierter } \\
\text { Hirnpotentiale (VEP, AEP, SSP) }\end{array}$ & 35,26 & 81,11 \\
\hline 1409 & Messung otoakustischer Emissionen & 23,31 & 53,62 \\
\hline
\end{tabular}




\section{Pathologie}

Im Rahmen von zahlreichen Leistungen auf Patientenwunsch (z. B. Endoskopien, Abstriche, Entfernung verdächtiger Hautbezirke, Operationen) werden Materialien gewonnen und diese im Rahmen von Überweisungen zur zytologischen oder histologischen Untersuchung zu Pathologen geschickt. Diese Überweisungen sollten mit den Patienten abgesprochen werden.

Als IGeL-Leistungen bieten Pathologen u. a. an:

Histologie

- Exidat, seborrhoische Keratose

- Lipom

- Ductus deferens Resektate

- Entnahme und Aufbereitung von Material von der Haut für die histologische Untersuchung

Zytologie

- Untersuchung zur Gebärmutterhals-Vorsorge für die Dünnschicht-Präparation (PapSpin)

- Zytologische Aufarbeitung von Material vom Gebärmutterhals für die immunzytochemische Untersuchung auf humane Papilloma Viren mit dem Cytoactiv-Test - IGEL-Bereich der Pathologen sind ca. 55\% der molekularpathologischen Leistungen durch HPV-Nachweise bedingt Dünnschichtzytologie zusätzlich zu der ", normalen“"Vorsorgezytologie

\section{Abrechnung}

Gemäß den Bestimmungen der GOÄ werden in der Regel alle diese Leistungen direkt vom Pathologen oder dem Arzt mit entsprechender Genehmigung zur Leistungserbringung abgerechnet und nicht vom Einsender oder Operateur. 


\section{Psychiatrische und psychotherapeutische Angebote}

\section{Was bieten die Fachgruppen der Psychiater und ärztliche Psychotherapeuten an IGeL-Leis-} tungen an?

Die Leistungsangebote der beiden Fachgruppen sind in großen Teilen identisch, so dass die Autoren die Angebote zusammengefasst haben.

- Angststörungen (Phobien, Panikattacken, soziale Ängste/Unsicherheiten, Prüfungsangst)

- Autogenes Training

- Biofeedback-BehandlungUntersuchung und Überprüfung des intellektuellen und psychosozialen Leistungsniveaus (Schullaufbahnberatung)

- Brain-Check

- Cranio-sacrale Therapie?

- Testpsychologisches Demenzscreening

- Depression, Transkranielle Magnetstimulation - z. B. Behandlung anhand von bestimmtem Licht (Bright-Light-Therapie)

- Essstörungen (Anorexie, Bulimie, Adipositas)

- Familientherapie

- Flugangsttherapie

- Gesprächspsychotherapie (GT

- Gutachten gemäß FeV (Führerschein)

- Hypnose

- Akute Krisensituationen (Trennung, Lebenskrisen, Beziehungsprobleme, Trauerproblematik, Posttraumatische Belastungsstörungen))

- Kunsttherapie und Körpertherapie

- Licht-Therapie z. B. bei Winterdepression und Schlafstörungen

- Migränebehandlung Migränebehandlung außerhalb der GKV-Leistungspflicht

- Muskelfunktionsanalyse

- Hilfe bei Personalauswahl/Personalberatung

- Konflikte in der Paarbeziehung (Umgang mit seelischen Verletzungen, Probleme in der Sexualität, Beziehungen außerhalb der Partnerschaft)

- Paartherapie

- Progressive Muskelentspannung

- Psychotherapie mit Richtlinien-Verfahren

- Psychotherapeutische Verfahren zur Selbsterfahrung

- Psychische Beschwerden im Zusammenhang mit körperlichen Erkrankungen

- Schlafstörungen

- Selbstbehauptungstraining

- Stress/Burn-Out und berufliche Probleme (z. B. Mobbing)

- Stressbewältigungstraining

- Suchterkrankungen (z. B. Spiel- oder Internetsucht; Rückfallprävention bei Alkoholabhängigkeit)

- Tiergestützte Therapie (Einsatz von Therapiehunden) bei behinderten Kindern oder Jugendlichen oder anderen Symptomen (z. B. Ängste, Unsicherheiten, Selbstwertprobleme)

- Beratung bei therapieresistentem erheblichem Übergewicht: a) Einzelberatung b) Kompaktkurs mind. 4 Teilnehmer, 6 Sitzungen, 4-wöchig, Anmeldung

- Verhaltenstherapie

- Zwangserkrankungen (Zwangsgedanken, Zwangshandlungen z. B. Wasch- oder Kontrollzwänge)

Psychotherapeutische Leistungen werden von Allgemeinmedizinern, Internisten, Gynäkologen, Neurologen, Nervenärzten, Psychiatern und ärztlichen Psychotherapeuten mit unterschiedlichen Qualifikationen angeboten.

Zahlreiche psychotherapeutische und/oder verhaltenstherapeutische Techniken werden von den GKV Kassen nicht gezahlt.

Ausgeschlossen sind z. B.

- Gesprächs-Psychotherapie

- Gestalttherapie

- Logotherapie

- Psychodrama

- Respiratorisches Biofeedback

- Transaktionsanalyse 
E. Individuelle Gesundheitsleistungen von A - Z

In der GKV regeln die Psychotherapie-Richtlinien des Bundesausschusses für Ärzte und Krankenkassen die Indikationen und Verfahren der Psychotherapie. Auch im Rahmen der Beihilfe sind zahlreiche Methoden ausgeschlossen - siehe Seite 58

Ausgeschlossen ist eine Psychotherapie in der GKV, wenn sie

- nicht der Heilung oder Besserung einer seelischen Krankheit dient, sondern nur zur besseren beruflichen und/oder sozialen Anpassung des Patienten;

- nur zu einer Erziehungs-, Ehe-, Lebens- oder Sexualberatung Anwendung findet.

Die eben genannten, bei der GKV ausgeschlossenen Anwendungsbereiche sind die Ansatzpunkte für IGEL-Angebote:

- Verbesserung der beruflichen Anpassung

- Verbesserung der sozialen Anpassung

- Erziehungsberatung

- Eheberatung

- Lebensberatung

- Konfliktberatung

- Sexualberatung

- Selbsterfahrung

- Stress-Problematik (Erkennen, Bewältigen)

Brück (Kommentar zur GOÄ) führt als Beispiele für psychotherapeutische Verfahren außerhalb der GKV-Leistungspflicht folgende Therapien an:

1. Humanistische Therapien

- Gestalttherapie

- Gesprächspsychotherapie

- Transaktionsanalyse

- Psychodrama

- Körper- und Bewegungstherapie

- Kunst-, Tanz- und Musiktherapie

2. Psychodynamische Therapie

- Hochfrequente Psychoanalyse **

3. Kognitiv-behaviorale Therapien

- Biofeedback

- Selbstbehauptungstraining

- Stressbewältigungstraining

- Sexualtherapie

4. Interpersonelle Therapien

- Familientherapie

- Paartherapie

\section{Entspannungsverfahren}

- Konzentrierte Meditation

** diese Therapie wird bei Vorliegen einer Erkrankung und erfolgreichem Antrag auf Psychoanalyse in der Regel von der GKV gezahlt

\section{Leistungen vor oder im Rahmen von psychotherapeutischen Verfahren}

In der Regel sind vor der Einleitung von psychotherapeutischen Verfahren Beratungen, Erörterungen und Untersuchungen erforderlich. Die Leistungen sind bei den folgenden Methoden nicht angegeben, sollten aber nicht vergessen werden!

\begin{tabular}{|l|l|r|r|}
\hline GOÄ Nr. & Kurzlegende & $\mathbf{1}$ fach $€$ & $\mathbf{2 , 3 f a c h} €$ \\
\hline $\mathbf{1}$ & Beratung & 4,66 & $\mathbf{1 0 , 7 2}$ \\
\hline $\mathbf{3}$ & $\begin{array}{l}\text { Eingehende Beratung (mind. 10 Min.) - nicht neben Sonderleis- } \\
\text { tungen }\end{array}$ & 8,74 & $\mathbf{2 0 , 1 1}$ \\
\hline $\mathbf{5}$ & Sympt. bezogene Untersuchung & 4,66 & $\mathbf{1 0 , 7 2}$ \\
\hline
\end{tabular}




\begin{tabular}{|c|c|c|c|}
\hline GOÄ Nr. & Kurzlegende & 1 fach $€$ & $2,3 f a c h €$ \\
\hline 8 & $\begin{array}{l}\text { Ganzkörperstatus } \\
\text { Neben Nr. } 8 \text { sind u. a. die GOÄ Nrn. 800, } 801 \text { etc. nicht berech- } \\
\text { nungsfähig }\end{array}$ & 15,15 & 34,85 \\
\hline 800 & Eingehende neurologische Untersuchung & 11,37 & 26,14 \\
\hline 801 & $\begin{array}{l}\text { Eingehende psychiatrische Untersuchung - ggf. unter Einschal- } \\
\text { tung der Bezugs- und/oder Kontaktperson } \\
\text { Neben Nr. } 801 \text { sind u. a. die GOÄ Nrn. 4, 8, } 807 \text { etc. nicht be- } \\
\text { rechnungsfähig }\end{array}$ & 14,57 & 33,52 \\
\hline 807 & $\begin{array}{l}\text { Erhebung einer biographischen psychiatrischen Anamnese bei } \\
\text { Kindern o. Jugendlichen unter Einschaltung der Bezugs- und } \\
\text { Kontaktpersonen mit schriftl. Aufzeichnung, auch in mehreren } \\
\text { Sitzungen } \\
\text { Neben Nr. } 807 \text { sind u. a. die GOÄ Nrn. 1, 3, 4, 22, 30, 34, } 801 \\
\text { etc. nicht berechnungsfähig }\end{array}$ & 23,31 & 53,62 \\
\hline 34 & Erörterung mind. 20 Min. & 17,49 & 40,2 \\
\hline
\end{tabular}

\section{Anti-Stress-Beratung}

\begin{tabular}{|l|l|r|r|}
\hline GOÄ Nr. & Kurzlegende & $\mathbf{1}$ fach $€$ & $\mathbf{2 , 3 f a c h} €$ \\
\hline $\mathbf{3}$ & $\begin{array}{l}\text { Eingehende Beratung (mind. 10 Min.) - nicht neben Sonderleis- } \\
\text { tungen }\end{array}$ & 8,74 & $\mathbf{2 0 , 1 1}$ \\
\hline $\mathbf{1}$ & Beratung & 4,66 & $\mathbf{1 0 , 7 2}$ \\
\hline $\mathbf{8}$ & Ganzkörperstatus & 15,15 & $\mathbf{3 4 , \mathbf { 8 5 }}$ \\
\hline $\mathbf{3 4}$ & Erörterung mind. 20 Min. & 17,49 & $\mathbf{4 0 , 2 3}$ \\
\hline $\mathbf{7 7}$ & Kurplanung & 8,74 & $\mathbf{1 0 , 1 0}$ \\
\hline $\mathbf{2 0}$ & Beratungsgespräch in Gruppen & 34,95 & $\mathbf{8 0 , 4 0}$ \\
\hline $\mathbf{8 5 7}$ & Orientierende Testuntersuchungen & 6,76 & $\mathbf{1 5 , 5 5}$ \\
\hline
\end{tabular}

Stress im Beruf und in der Familie ist heute nicht gerade selten. Angebote zur Stressbewältigung z. B.

- Biofeedback

- Quigong

- Feldenkrais

- Musiktherapie

- Farbtherapie

sind nach den Nrn. 846 oder 847 zu berechnen.

Stress-Bewältigungstraining

\begin{tabular}{|l|l|r|r|}
\hline GOÄ Nr. & Kurzlegende & $\mathbf{1 f a c h} € \mathbf{2 , 3 f a c h ~} \mathbf{~}$ \\
\hline $\mathbf{8 4 6}$ & Stressbewältigungstraining - Einzelbeh. z. B. autogenes Training & 8,74 & $\mathbf{2 0 , 1 1}$ \\
\hline $\mathbf{8 4 7}$ & $\begin{array}{l}\text { Stressbewältigungstraining - Gruppenbeh. z. B. autogenes } \\
\text { Training }\end{array}$ & 2,62 & $\mathbf{6 , 0 3}$ \\
\hline $\mathbf{8 7 0}$ & Verhaltenstherapie, Einzelbehandlung mind. 50 Min. & 43,72 & $\mathbf{1 0 0 , 5 5}$ \\
\hline $\mathbf{8 7 1}$ & Verhaltenstherapie, Gruppenbehandlung mind. 50 Min. & 8,74 & $\mathbf{2 0 , 1 1}$ \\
\hline
\end{tabular}

Psychotherapeutische Verfahren zur Selbsterfahrung ohne medizinische Indikation

\begin{tabular}{|l|l|r|r|}
\hline GOÄ Nr. & Kurzlegende & $\mathbf{1 f a c h} €$ & $\mathbf{2 , 3 f a c h} €$ \\
\hline $\mathbf{8 6 0}$ & Erhebung einer biographischen Anamnese & 53,62 & $\mathbf{1 2 3 , 3 4}$ \\
\hline $\begin{array}{l}\mathbf{8 4 6} \\
\text { analog }\end{array}$ & $\begin{array}{l}\text { Psychotherapeutische Verfahren zur Selbsterfahrung ohne medi- } \\
\text { zinische Indikation - analog GOÄ Nr. 846 - entspr. GOÄ § 6 (2) } \\
\text { Empfehlung der BÄK - GOÄ Kurztext Nr. 846: Übende Verfahren }\end{array}$ & 8,74 & $\mathbf{2 0 , 1 1}$ \\
\hline
\end{tabular}


E. Individuelle Gesundheitsleistungen von A - Z

\begin{tabular}{|l|l|r|r|}
\hline GOÄ Nr. & Kurzlegende & $\mathbf{1 f a c h} €$ & $\mathbf{2 , 3 f a c h ~} \mathbf{}$ \\
\hline $\begin{array}{l}\mathbf{8 4 7} \\
\text { analog }\end{array}$ & $\begin{array}{l}\text { Übende Verfahren, Gruppenbehandlung, mind. 20 Min., höchs- } \\
\text { tens 12 Teilnehmer, je Teilnehmer - analog GOÄ Nr. 847 - GOÄ } \\
\text { § 6 (2) })^{1)} \\
\text { GOÄ Kurztext Nr. 847: Übende Verfahren in Gruppenbehandlung }\end{array}$ & 2,62 \\
\hline $\mathbf{8 7 0}$ & Verhaltenstherapie, Einzelbehandlung mind. 50 Min. & $\mathbf{6 , 0 3}$ \\
\hline $\mathbf{8 7 1}$ & Verhaltenstherapie, Gruppenbehandlung mind. 50 Min. & 8,72 & $\mathbf{1 0 0 , 5 5}$ \\
\hline
\end{tabular}

1) Bei analogen Leistungen muss - mind. in Kurzform - auch die Original-Leistungslegende angegeben werden.

Selbstbehauptungstraining

Unterschiedliche Methoden werden unter diesem Begriff angeboten. Alle Verfahren arbeiten mit Rollenspielen, Verhaltensübungen, Modell-Lernen und/oder Feedback-Methoden.

\begin{tabular}{|c|c|c|c|}
\hline GOÄ Nr. & Kurzlegende & 1fach $€$ & 2,3 fach $€$ \\
\hline $\begin{array}{l}846 \\
\text { analog }\end{array}$ & $\begin{array}{l}\text { Selbstbehauptungstraining - analog GOÄ Nr. } 846 \text { - entspr. GOÄ } \\
\S 6(2)^{1)} \\
\text { Empfehlung der BÄK - GOÄ Kurztext Nr. 846: Übende Verfahren }\end{array}$ & 8,74 & 20,11 \\
\hline $\begin{array}{l}847 \\
\text { analog }\end{array}$ & $\begin{array}{l}\text { Übende Verfahren, Gruppenbehandlung, mind. } 20 \text { Min., höchs- } \\
\text { tens } 12 \text { Teilnehmer, je Teilnehmer - analog GOÄ Nr. } 847 \text { - } \\
\text { entspr. GOÄ } \S 6(2)^{1)} \\
\text { GOÄ Kurztext Nr. 847: Ubbende Verfahren in Gruppenbehandlung }\end{array}$ & 2,62 & 6,03 \\
\hline 870 & Verhaltenstherapie, Einzelbehandlung mind. 50 Min. & 43,72 & 100,55 \\
\hline 871 & Verhaltenstherapie, Gruppenbehandlung mind. 50 Min. & 8,74 & 20,11 \\
\hline
\end{tabular}

1) Bei analogen Leistungen muss - mind. in Kurzform - auch die Original-Leistungslegende angegeben werden.

Entspannungsverfahren als Präventionsleistung

Zu den bekannten Entspannungsverfahren zählen:

- Autogenes Training

- Progressive Muskelentspannung nach Jacobsen

- Konzentrative Meditation

Alle 3 Verfahren werden nach den GOÄ Nrn. 846 und 847 abgerechnet.

\begin{tabular}{|l|l|r|r|}
\hline GOÄ Nr. & Kurzlegende & $\mathbf{1 f a c h} €$ & $\mathbf{2 , 3 f a c h} €$ \\
\hline $\mathbf{8 4 6}$ & Entspannungsverfahren als Präventionsleistung, Einzelbeh. & 8,74 & $\mathbf{2 0 , 1 1}$ \\
\hline $\mathbf{8 4 7}$ & Entspannungsverfahren als Präventionsleistung, Gruppenbeh. & 2,62 & $\mathbf{6 , 0 3}$ \\
\hline
\end{tabular}

Klangsessel

\begin{tabular}{|l|l|r|r|}
\hline $\begin{array}{l}\mathbf{8 4 6} \\
\text { analog }\end{array}$ & $\begin{array}{l}\text { Biofeedback } \\
\text { analog Nr. 846 GOÄ - entsprechend GOÄ § 6 (2) }\end{array}$ & 8,74 & $\mathbf{2 0 , 1 1}$ \\
\hline
\end{tabular}

Myofeedback-Behandlung

\begin{tabular}{|l|l|r|r|}
\hline $\begin{array}{l}\mathbf{8 4 6} \\
\text { analog }\end{array}$ & $\begin{array}{l}\text { Myofeedback } \\
\text { analog Nr. 846 GOÄ - entsprechend GOÄ § 6 (2) }\end{array}$ & 8,74 & $\mathbf{2 0 , 1 1}$ \\
\hline
\end{tabular}

Neurofeedback-Entspannungsbehandlung

\begin{tabular}{|l|l|r|r|}
\hline $\begin{array}{l}\mathbf{8} 46 \\
\text { analog }\end{array}$ & $\begin{array}{l}\text { Neurofeedback } \\
\text { analog Nr. 846 GOÄ - entsprechend GOÄ § 6 (2) }\end{array}$ & 8,74 & $\mathbf{2 0 , 1 1}$ \\
\hline
\end{tabular}


Hypnose

\begin{tabular}{|l|l|r|r|}
\hline GOÄ-Nr. & Kurzlegende & $\mathbf{1 f a c h} €$ & $\begin{array}{r}\text { *1,15/ } \\
\mathbf{2 , 3 f a c h} €\end{array}$ \\
\hline $\mathbf{1}$ & Beratung, auch tel. & 4,66 & $\mathbf{1 0 , 7 3}$ \\
\hline $\mathbf{3}$ & $\begin{array}{l}\text { Eingehende Beratung, auch tel. (mind. 10 Min.) nicht neben Son- } \\
\text { derleistungen }\end{array}$ & 8,74 & $\mathbf{2 0 , 1 1}$ \\
\hline $\mathbf{8 4 5}$ & Behandlung einer Einzelperson durch Hypnose & 8,74 & $\mathbf{2 0 , 1 1}$ \\
\hline
\end{tabular}

Biofeedback-Behandlung

Indikationen zu einer Biofeedback-Behandlung auf Patientenwunsch sind nach Brück (Kommentar zur GOÄ) z. B.

- Spannungskopfschmerz

- Migräne

- Schlafstörungen

- chron. Schmerzzustände

- Tinnitus

- Epilepsie

- Depressionen

- Nächtliches Zähneknirschen (Bruxismus)

- Angstanfälle

- Schlaganfall

Angewandte Biofeedback-Verfahren:

- Respiratorisches oder Atem-Biofeedback

- EMG-Feedback

- Hauttemperatur-Feedback

- Vasomotorisches-Feedback

- Hautwiderstands-Feedback

- Blutdruck-Feedback

- Herzfrequenz-Feedback

- EEG-Feedback

\begin{tabular}{|l|l|r|r|}
\hline GOÄ Nr. & Kurzlegende & $\mathbf{1 f a c h ~ € ~}$ & $\mathbf{2 , 3 f a c h ~ € ~}$ \\
\hline $\begin{array}{l}\mathbf{8 4 6} \\
\text { analog }\end{array}$ & $\begin{array}{l}\text { Biofeedback-Behandlung-Einzelbehandl. - analog GOÄ Nr. 846 } \\
\text { - entspr. GOÄ § 6 (2) } \\
\text { Empfehlung der BÄK - GÖ̈ Kurztext Nr. 846: Übende Verfahren }\end{array}$ & $\mathbf{8 , 7 4}$ \\
\hline $\begin{array}{l}\mathbf{8 4 7} \\
\text { analog }\end{array}$ & $\begin{array}{l}\text { Biofeedback-Behandlung-Gruppenbehandl. - analog GOÄ Nr. 847 } \\
- \text { entspr. GOÄ § 6 (2)1) } \\
\text { GOÄ Kurztext Nr. 847: Übende Verfahren in Gruppenbehandlung }\end{array}$ & $\mathbf{2 , 6 2}$ \\
\hline
\end{tabular}

1) Bei analogen Leistungen muss - mind. in Kurzform - auch die Original-Leistungslegende angegeben werden.

Abrechnungshinweis: Sowohl für das Atem-Biofeedback als auch für das EMG-Biofeedback wird die Nr. 846 analog angesetzt. Wegen des hohen apparativen Aufwandes erscheint ein höherer Steigerungsfaktor begründbar.

Kunst- und Körpertherapien - auch als ergänzende Therapieverfahren

\begin{tabular}{|l|l|r|r|}
\hline GOÄ Nr. & Kurzlegende & $\mathbf{1 f a c h ~} \mathbf{2 , 3 f a c h ~} \mathbf{~}$ \\
\hline $\begin{array}{l}\mathbf{8 6 1} \\
\text { analog }\end{array}$ & $\begin{array}{l}\text { Kunst- U. Körpertherapien - Einzeltherapie - analog GOÄ } \\
\text { Nr. 861-entspr. GOÄ § 6 (2) } \\
\text { GOÄ Kurztext Nr. 861: Tiefenpsychologisch fundierte Psychotherapie, Einzel- } \\
\text { behandlung... }\end{array}$ & $\mathbf{9 2 , 5 0}$ \\
\hline $\begin{array}{l}\mathbf{8 6 2} \\
\text { analog }\end{array}$ & $\begin{array}{l}\text { Kunst- U. Körpertherapien - Gruppentherapie - analog GOÄ } \\
\text { Nr. 862 - entspr. GOÄ § 6 (2) }\end{array}$ & $\begin{array}{l}\text { 1) } \\
\text { GOÄ Kurztext Nr. 862: Tiefenpsychologisch fundierte Psychotherapie, Gruppen- } \\
\text { behandlung... }\end{array}$ & $\mathbf{4 6 , 2 5}$ \\
\hline
\end{tabular}




\begin{tabular}{|l|l|r|r|}
\hline GOÄ Nr. & Kurzlegende & $\mathbf{1 f a c h ~} €$ & $\mathbf{2 , 3 f a c h ~ € ~}$ \\
\hline $\begin{array}{l}\mathbf{8 4 6} \\
\text { analog }\end{array}$ & $\begin{array}{l}\text { Kunst- und Körpertherapien auch als ergänzende Therapiever- } \\
\text { fahren - Einzeltherapie - analog GOÄ Nr. 846 - entspr. GOÄ § 6 } \\
(2)^{1)} \\
\text { Empfehlung der BÄK - GOÄ Kurztext Nr. 846: Übende Verfahren }\end{array}$ & $\mathbf{8 , 7 4}$ & $\mathbf{2 0 , 1 1}$ \\
\hline $\begin{array}{l}\mathbf{8 4 7} \\
\text { analog }\end{array}$ & $\begin{array}{l}\text { Kunst- U. Körpertherapien - Gruppentherapie - analog GOÄ } \\
\text { Nr. 847 - entspr. GOÄ § 6 (2) } \\
\text { GOÄ Kurztext Nr. 847: Übende Verfahren in Gruppenbehandlung }\end{array}$ & 2,62 & $\mathbf{6 , 0 3}$ \\
\hline
\end{tabular}

1) Bei analogen Leistungen muss - mind. in Kurzform - auch die Original-Leistungslegende angegeben werden.

\section{Verhaltenstherapie bei Flugangst}

\begin{tabular}{|l|l|r|r|}
\hline GOÄ Nr. & Kurzlegende & $\mathbf{1 f a c h} €$ & $\mathbf{2 , 3 f a c h} €$ \\
\hline $\begin{array}{l}\mathbf{8 4 9} \\
\text { analog }\end{array}$ & $\begin{array}{l}\text { Übende Verfahren bei Flugangst (mind. 20 Min.) - analog GOÄ } \\
\text { Nr. 849 - entspr. GOÄ § 6 (2) } \\
\text { GOÄ Kurztext Nr. 849: Psychotherapeutische Behandlung.... }\end{array}$ & 13,41 & $\mathbf{3 0 , 8 3}$ \\
\hline $\mathbf{8 7 0}$ & Verhaltenstherapie bei Flugangst, Einzelbehandlung mind. 50 Min. & 43,72 & $\mathbf{1 0 0 , 5 5}$ \\
\hline $\mathbf{8 7 1}$ & Verhaltenstherapie bei Flugangst, Gruppentherapie mind. 50 Min. & 8,74 & $\mathbf{2 0 , 1 1}$ \\
\hline
\end{tabular}

1) Bei analogen Leistungen muss - mind. in Kurzform - auch die Original-Leistungslegende angegeben werden. 


\section{Radiologie}

Was bietet die Fachgruppe als IGeL-Leistungen an?

- Arterosklerose-Check-Up: MR-Angiographie zur frühzeitigen

Erkennung eventueller Arterosklerose-Anzeichen

- Brust-Implantat-Diagnostik mit MR-Mammographie

- Brustkrebsvorsorge: Röntgen-Mammographie, Ultraschall, MR-Mammographie

- Blutgefäße - Doppler-Untersuchungen z. B. Extremitäten, Halsschlagader, Hirngefäße

- demenzieller Erkrankungen: Funktionelles MRT zur Früherkennung

- Ganzkörper-MRT: zur frühzeitigen Erkennung von Problemen in Gehirn, Knochen und inneren Organen

- Lungenkrebsvorsorge: Lungenscreening zum Ausschluss eines Lungentumors

- Osteoporosecheck: Knochendichtemessung zur Früherkennung von Osteoporose

- Schlaganfallprophylaxe: MRT des Kopfes ohne Bestrahlung

zur Verminderung des Schlaganfallrisikos

- Ultraschall-Check-up: z.B.: der abdominalen Organe, der Nieren, der Schilddrüse, Prostata

Patienten vor Überweisung über die Kosten informieren

Ein Arzt, der seine Patienten im Rahmen von IGeL-Leistungen zum Radiologen überweist, muss den Patienten auf die zu erwartenden (durchaus erheblichen) Kosten hinweisen. Eine radiologische Praxis informiert Patienten über die Kosten einiger ihrer Leistungen:

.... „Die anfallenden Kosten entsprechen dem einfachen Abrechnungssatz der Gebührenordnung für Ärzte. Sie betragen für:

- Herzinfarktvorsorge mit Befundgespräch 480,-- Euro

- Schlaganfallprophylaxe (Angiographie und Hirnuntersuchung) 350,- Euro

- Gefäßvorsorge (z. B. Bein) 350,- Euro

- Krebsfrüherkennungsprophylaxe (z. B. Brust, Prostata) 380,- Euro

- Separate Kontrastmitteleinbringung 60,-Euro ..."

Indikationen für die MRM als GKV-Leistung

Das Radiologen WirtschaftsForum Ausgabe 7/2012 (http://www.radiologen-foren.de/rwf/node/ 856) informiert zum Problem der Abrechnung:

.... „Der Gemeinsame Bundesausschuss (G-BA) hat die MRM in die Richtlinien „Methoden vertragsärztliche Versorgung“ in die Anlage 1 „Anerkannte Untersuchungs- oder Behandlungsmethoden“ aufgenommen. Die in der Anlage 1 festgelegten Indikationen wurden in die Leistungslegende zu Nr. 34431 übernommen. MRM sind demnach zulasten der GKV nur berechnungsfähig

- zum Rezidivausschluss (frühestens sechs Monate nach der Operation oder zwölf Monate nach Beendigung der Bestrahlungstherapie) eines histologisch gesicherten Mammakarzinoms nach brusterhaltender Therapie,

- nach Wiederaufbauplastik für den Fall, dass eine vorausgegangene mammographische und sonographische Untersuchung die Dignität des Rezidivverdachtes nicht klären konnte oder

- zur Primärtumorsuche bei axillären Lymphknotenmetastasen, deren histologische Morphologie ein Mammakarzinom nicht ausschließt, wenn ein Primärtumor weder klinisch noch mittels mammographischer und sonographischer Untersuchung dargestellt werden konnte.

Voraussetzung für die MRM ist somit, dass jeweils vorab durch eine Mammographie und Sonographie der Versuch einer weiteren Abklärung durchgeführt wird. Die mammographische bzw. sonographische Untersuchung muss allerdings nicht zwingend durch den Radiologen vorgenommen werden, der die MRM erbringt. Hier können auch die Untersuchungsergebnisse anderer Ärzte als Grundlage für die MRM beigezogen werden.

Paradox: Laut Leistungslegende ist die Nr. 34431 nur für die MRM der weiblichen Brustdrüse berechnungsfähig. Streng genommen können somit die sehr seltenen Fälle eines Mamma-CA bei Männern nicht dieser Untersuchung zugeführt werden. Das sollte aber niemanden davon abhalten, bei entsprechender Indikation dennoch die Nr. 34441 bei Männern abzurechnen. In der Regel gibt es dann keine Erstattungsprobleme. ...

Radiologen können von GKV-Patienten gemäß Bundesmantelvertrag (BMV) nur auf Überweisung eines anderen Vertragsarztes in Anspruch genommen werden. Selten kommen Patienten direkt zum Radiologen, um bestimmte Wunschleistungen zu verlangen. 


\section{E. Individuelle Gesundheitsleistungen von A - Z}

Um späteren Problemen bei der Abrechnung zu entgehen, sollte der Radiologe stets prüfen, ob der Patient die Untersuchung zur Abklärung einer Erkrankung wünscht und, wenn dies der Fall ist, daraufhin weisen, dass die Untersuchung ggf. auf Überweisung durch einen anderen Vertragsarzt (z. B.Hausarzt, Internisten, Gynäkologen) als Kassenleistung erfolgen könnte. Möglicherweise ist eine telefonische Rücksprache mit dem behandelnden Arzt sinnvoll und erbringt die erforderliche Überweisung.

Werden vom Patienten Wunschuntersuchungen erbeten oder mit einem Überweisungsschein radiologische Untersuchungen verlangt, die nicht als vertragsärztliche Leistungen berechnungsfähig sind - z. B. eine MRT-Untersuchung der Herzkranzgefäße - muss der Patient aufgeklärt werden, dass die erwünschte Untersuchung nur gegen Privatliquidation (als IGEL-Leistung) erfolgen kann. Mit dem Patienten sollte dann ein entsprechender Vertrag geschlossenen werden, der deutlich macht, dass es sich um eine Wunschleistung auf eigene Kosten des Patienten handelt und nicht um eine GKV-Leistung. Ferner sollten im Vertrag die verabredeten Leistungen und die Kosten nach GOÄ mit Steigerungssätzen angegeben sein.

Früherkennung Osteoporose (Knochendichtemessung)

Untersuchung auf Wunsch des Patienten/der Patientin ohne einen aktuellen Krankheitsverdacht.

\begin{tabular}{|l|l|r|r|}
\hline GOÄ Nr. & Kurzlegende & $\mathbf{1 f a c h} €$ & $\begin{array}{r}{ }^{*} \mathbf{1 , 8 /} \\
\mathbf{2 , 3 f a c h} €\end{array}$ \\
\hline $\mathbf{1}$ & Beratung & 4,66 & $\mathbf{1 0 , 7 2}$ \\
\hline $\mathbf{5 3 8 0 *}$ & $\begin{array}{l}\text { Osteodensitometrie von Skelettteilen mit quant. CT oder digitaler } \\
\text { Röntgentechnik }\end{array}$ & 17,49 & $\mathbf{3 1 , 4 8}$ \\
\hline
\end{tabular}

Lungenkrebsvorsorge (CT-Raucher-Screening)

Untersuchung auf Wunsch des Patienten/der Patientin ohne einen aktuellen Krankheitsverdacht.

Eine radiologische Praxis in Berlin informiert zu dieser angebotenen Leistung Patienten mit folgendem Text:

\section{Lungenkrebsvorsorge (CT-Raucher-Screening)}

Wenn Sie z. B. als Raucher mit deutlich erhöhtem Lungenkrebsrisiko den möglichst sicheren Ausschluss eines Lungentumors wünschen, sollten Sie ein Lungenscreening mit Niedrig-DosisCT des Thorax durchführen lassen. Damit lässt sich auch ein kleiner noch symptomloser Raucher-Lungenkrebs in allen Abschnitten der Lunge deutlich besser entdecken als mit den konventionellen Röntgenaufnahmen des Thorax. Dabei erfahren Sie dann auch, ob und wie stark der Nikotinkonsum Ihre Lunge oder die Bronchien chronisch geschädigt hat.

Während das Lungenscreening mit CT selbst kleinere Veränderungen des Lungengewebes und des Rippenfells zu Tage fördert, können gegebenenfalls im Rahmen der virtuellen Bronchoskopie, die ebenfalls auf der Multislice-Technologie basiert, bereits kleinste Auffälligkeiten auch innerhalb der Bronchien erkannt und beurteilt werden. Beide Methoden sind sehr strahlungsarm, da die Lunge nur wenige Röntgenstrahlen absorbiert.

\begin{tabular}{|l|l|r|r|}
\hline GOÄ Nr. & Kurzlegende & $\mathbf{1 f a c h} €$ & $\begin{array}{r}{ }^{*} \mathbf{1 , 8 /} \\
\mathbf{2 , 3 f a c h} \boldsymbol{\ddagger}\end{array}$ \\
\hline $\mathbf{1}$ & Beratung & 4,66 & $\mathbf{1 0 , 7 2}$ \\
\hline $\mathbf{5 3 7 1}^{*}$ & CT im Thoraxbereich & 134,06 & $\mathbf{2 4 1 , 3 1}$ \\
\hline $\mathbf{5 3 7 6}^{*}$ & CT-Ergänzung, High-Resolution- Technik & 29,14 & $\mathbf{5 2 , 4 6}$ \\
\hline $\mathbf{5 3 7 7}^{*}$ & $\begin{array}{l}\text { Computergestützte Analyse mit 3-D-Rekonstruktion - nur 1facher } \\
\text { Satz möglich }\end{array}$ & $\mathbf{4 6 , 6 3}$ & - \\
\hline
\end{tabular}


Schlaganfall-Prophylaxe

Untersuchung auf Wunsch des Patienten/der Patientin ohne einen aktuellen Krankheitsverdacht.

\begin{tabular}{|c|c|c|c|}
\hline GOÄ Nr. & Kurzlegende & 1 fach $€$ & $\begin{array}{r}{ }^{*} 1,8 / \\
2,3 f a c h €\end{array}$ \\
\hline 1 & Beratung & 4,66 & 10,72 \\
\hline $5700^{*}$ & MRT im Kopfbereich inklusive Halsregion & 256,46 & 461,64 \\
\hline $5731^{*}$ & ergänzende Serie, MR-Angiographie & 58,29 & 104,92 \\
\hline 5733* & $\begin{array}{l}\text { computergestützte Analyse mit 3-D-Rekonstruktion -nur 1facher } \\
\text { Satz möglich }\end{array}$ & 46,63 & - \\
\hline 346 & Hochdruck-Kontrastmittel-Injektion & 17,49 & 40,22 \\
\hline
\end{tabular}

Auslagen: MRT-Kontrastmittel Berechnung nach GOÄ § 10

Arteriosklerose-Check-up

Untersuchung auf Wunsch des Patienten/der Patientin ohne einen aktuellen Krankheitsverdacht.

1. MR-Angiographie der Becken-Bein-Arterien

\begin{tabular}{|l|l|r|r|}
\hline GOÄ Nr. & Kurzlegende & $\mathbf{1 f a c h} \boldsymbol{€}$ & $\begin{array}{r}\mathbf{1}, \mathbf{8} / \\
\mathbf{2 , 3 f a c h} \boldsymbol{€}\end{array}$ \\
\hline $\mathbf{1}$ & Beratung & 4,66 & $\mathbf{1 0 , 7 2}$ \\
\hline $\mathbf{5 7 3 0}$ & MRT von ganzen Extremitäten & 233,15 & $\mathbf{4 1 9 , 6 7}$ \\
\hline $\mathbf{5 7 3 1}^{*}$ & ergänzende Serie, MR-Angiographie & 58,29 & $\mathbf{1 0 4 , 9 2}$ \\
\hline $\mathbf{5 7 3}^{*}$ & computergestützte Analyse & $\mathbf{4 6 , 6 3}$ & - \\
\hline $\mathbf{3 4 6}^{*}$ & Hochdruck-Kontrastmittel-Injektion & 17,49 & $\mathbf{4 0 , 2 2}$ \\
\hline
\end{tabular}

Auslagen: MRT-Kontrastmittel, Berechnung nach GOÄ § 10

2. MR-Angiographie der Nieren

\begin{tabular}{|l|l|r|r|}
\hline GOÄ Nr. & Kurzlegende & $\mathbf{1 f a c h} \boldsymbol{€}$ & $\begin{array}{r}{ }^{*} \mathbf{1 , 8 /} \\
\mathbf{2 , 3 f a c h} \boldsymbol{€}\end{array}$ \\
\hline $\mathbf{1}$ & Beratung & 4,66 & $\mathbf{1 0 , 7 2}$ \\
\hline $\mathbf{5 7 2 0}^{\star}$ & MRT im Bereich des Abdomens & 256,46 & - \\
\hline $\mathbf{5 7 3 1 ^ { * }}$ & ergänzende Serie, MR-Angiographie & 58,29 & $\mathbf{1 0 4 , 9 2}$ \\
\hline $\mathbf{5 7 3 3}^{\star}$ & computergestützte Analyse - nur 1facher Satz möglich & $\mathbf{4 6 , 6 3}$ & - \\
\hline $\mathbf{3 4 6}^{*}$ & Hochdruck-Kontrastmittel-Injektion & 17,49 & $\mathbf{4 0 , 2 2}$ \\
\hline
\end{tabular}

Brustkrebsvorsorge mit Röntgen-Mammographie und Ultraschall

Untersuchung auf Wunsch der Patientin ohne einen aktuellen Krankheitsverdacht.

\begin{tabular}{|c|c|c|c|}
\hline GOÄ Nr. & Kurzlegende & 1 fach $€$ & $\begin{array}{r}{ }^{*} 1,8 / \\
2,3 \mathrm{fach} €\end{array}$ \\
\hline 1 & Beratung & 4,66 & 10,72 \\
\hline 5 & körperliche Untersuchung (Tastbefund) & 4,66 & 10,73 \\
\hline $5266^{*}$ & Mammographie einer Seite in 2 Ebenen & 26,23 & 47,21 \\
\hline $5266^{*}$ & Mammographie andere Seite in 2 Ebenen & 26,23 & 47,21 \\
\hline 418 & Ultraschall einer Brustdrüse & 12,24 & 28,15 \\
\hline 420 & Ultraschall 1 weiteres Organs - andere Brustdrüse & 4,66 & 10,73 \\
\hline
\end{tabular}


E. Individuelle Gesundheitsleistungen von A - Z

MR-Mammographie

Untersuchung auf Wunsch der Patientin ohne einen aktuellen Krankheitsverdacht.

\begin{tabular}{|l|l|r|r|}
\hline GOÄ Nr. & Kurzlegende & $\mathbf{1}$ fach $\boldsymbol{€}$ & $\begin{array}{r}{ }^{*} \mathbf{1 , 8 /} \\
\mathbf{2 , 3} \mathbf{f a c h} \boldsymbol{€}\end{array}$ \\
\hline $\mathbf{1}$ & Beratung & 4,66 & $\mathbf{1 0 , 7 2}$ \\
\hline $\mathbf{5}$ & körperliche Untersuchung (Tastbefund) & 4,66 & $\mathbf{1 0 , 7 3}$ \\
\hline $\mathbf{5 7 2 1 ^ { * }}$ & MRT der Mammae (Brust) & 233,15 & $\mathbf{4 1 9 , 6 7}$ \\
\hline $\mathbf{5 7 3 1 ^ { * }}$ & ergänzende Serie nach i.v. Kontrastmittel & 58,29 & $\mathbf{1 0 4 , 9 2}$ \\
\hline $\mathbf{5 7 3 3 ^ { * }}$ & computergestützte Analyse, Kinetik - nur 1facher Satz möglich & $\mathbf{4 6 , 6 3}$ & - \\
\hline $\mathbf{3 4 6}$ & Hochdruck-Kontrastmittel-Injektion & 17,49 & $\mathbf{4 0 , 2 2}$ \\
\hline
\end{tabular}

Auslagen: MRT-Kontrastmittel, Berechnung nach GOÄ § 10

Check-up der inneren Organe durch Ultraschall und/oder MRT

Untersuchung auf Wunsch des Patienten/der Patientin ohne einen aktuellen Krankheitsverdacht.

Ultraschall-Check-up

\begin{tabular}{|l|l|r|r|}
\hline GOÄ Nr. & Kurzlegende & $\mathbf{1 f a c h} €$ & $\begin{array}{r}{ }^{*} \mathbf{1 , 8 /} \\
\mathbf{2 , 3 f a c h} €\end{array}$ \\
\hline $\mathbf{1}$ & Beratung & 4,66 & $\mathbf{1 0 , 7 2}$ \\
\hline $\mathbf{5}$ & körperliche Untersuchung (Tastbefund) & 4,66 & $\mathbf{1 0 , 7 3}$ \\
\hline $\mathbf{4 1 0}$ & Ultraschalluntersuchung eines Organs & 25,64 & $\mathbf{2 6 , 8 1}$ \\
\hline $\mathbf{4 2 0}$ & Ultraschalluntersuchung bis zu 3 weiteren Organen -je Organ & 4,66 & $\mathbf{1 6 , 3 2}$ \\
\hline
\end{tabular}

MRT-Check-up

\begin{tabular}{|l|l|r|r|}
\hline GOÄ Nr. & Kurzlegende & $\mathbf{1 f a c h} \boldsymbol{€}$ & $\begin{array}{r}{ }^{*} \mathbf{1 , 8} / \\
\mathbf{2 , 3 f a c h} €\end{array}$ \\
\hline $\mathbf{1}$ & Beratung & 4,66 & $\mathbf{1 0 , 7 2}$ \\
\hline $\mathbf{5 7 2 0}$ & MRT im Bereich des Abdomens & 256,46 & $\mathbf{4 6 1 , 6 4}$ \\
\hline $\mathbf{5 7 3 1}$ & ergänzende Serie, MRCP & 58,29 & $\mathbf{1 0 4 , 9 2}$ \\
\hline $\mathbf{5 7 3 3}$ & computergestützte Analyse - nur 1facher Satz möglich & $\mathbf{4 6 , 6 3}$ & - \\
\hline
\end{tabular}

Die Radiologische Universitätsklinik Abteilung Röntgendiagnostik des Klinikums Freiburg bietet auf ihren Internetseiten (www.uniklinik-freiburg.de/ip/live/patientenservice/zusatzangebote/radio. html) weitere IGEL-Leistungen an und informiert Patienten darüber:

\section{Ganzkörper-Magnetresonanztomographie}

Möglichkeiten der Ganzkörper Magnetresonanztomographie sind:

- Nachweis oder Ausschluss von krankhaften Veränderungen

- des Lymphsystems - der Knochen - der Leber, der Milz und der Nieren

- Nachweis oder Ausschluss von Erkrankungen des Skelettsystems, insbesondere entzündlicher Veränderungen.

Das Verfahren ist derzeit nicht geeignet, um im gleichen Untersuchungsgang den Magen-Darm-

Trakt zu untersuchen. Hierfür sind spezielle Untersuchungssequenzen erforderlich.

\section{Darmkrebs rechtzeitig entdecken}

Darmkrebs ist heute bei rechtzeitiger Diagnose heilbar mit der neuen Multislice CT-Untersuchung.

Verfahren zur Linderung und Heilung -

Tumorbehandlung mit Minimalinvasiver Therapie durch Radio-Frequenz-Tumorzerstörung für bestimmte:

- Lebertumore

- Lungentumore

- Nierentumore 


\section{Urologie}

Was bietet die Fachgruppe als IGeL-Leistungen an?

- Behandlung von Erektionsstörungen

- Fruchtbarkeits-Check für inn

- Hormonbestimmung (Spermiogramm)

- Krebsfrüherkennung Nieren und Blase für die Frau

- Krebsfrüherkennung Nieren, Blase, Prostata und Hoden für den Mann

- Labor-Wunschleistungen (PSA-Analyse, Kontrolle des Urins auf bösartige Zellen

- Prostata-Check mit PSA-Test zur Früherkennung

- Sterilisation - ambulante Vasektomie in Lokalanästhesie oder Kurznarkose

- Wiederherstellung der Zeugungsfähigkeit nach Sterilisation durch mikrochirurgische Operation (Mikrochirurgische Vaso-Vasoneostomie)?

Intimästhetische/Intimchirurgische Leistungen für den Mann werden von Urologen und verstärkt von Plastischen Chirurgen angeboten, u. a.:

- Hodenprothese

- Hodenstraffung

- Penisverlängerung

- Penisverdickung

- Penisverkrümmung

- Penisverlängerung

- Phimosebehandlung/Beschneidung/Zirkumzision

- Sterilisation

- Versteckter Penis

\section{Komplett-Vorsorge für Männer}

Die Vorsorgeuntersuchungen für den Mann sind der oft erste Einstieg eines Patienten beim Urologen. Dazu kommt es, weil viele Patienten den Urologen mit seinen speziellen Kenntnissen und der apparativen Ausstattung (z. B. transrectale Sonographie) für besonders qualifiziert halten zur Prävention für den Mann.

Weitere Schwerpunkte sind

- Fruchtbarkeitscheck ohne Krankheitshinweis

- Behandlung von Potenzstörungen

\begin{tabular}{|c|c|c|c|}
\hline GOÄ Nr. & Kurzlegende & 1 fach $€$ & $\begin{array}{r}{ }^{*} 1,8 / \\
2,3 f a c h \\
\end{array}$ \\
\hline 5 & Symptombezogene Untersuchung & 4,66 & 10,73 \\
\hline 6 & $\begin{array}{l}\text { Untersuchung Nieren und harnableitende Wege, bei Männern } \\
\text { zusätzlich Prostata, Bruchpforten, Hoden und Nebenhoden }\end{array}$ & 5,83 & 13,41 \\
\hline 8 & Ganzkörperstatus & 15,15 & 34,85 \\
\hline 11 & Digitale Untersuchung von Mastdarm u./o. Prostata & 3,50 & 8,04 \\
\hline 28 & Krebsvorsorge: Mann & 16,32 & 37,54 \\
\hline $403^{*}$ & Zuschlag zur Sonographie bei transrectaler Untersuchung & 8,74 & 15,74 \\
\hline 410 & Sonographie, ein Organ (Prostata) & 11,66 & 26,81 \\
\hline \multirow[t]{2}{*}{420} & Sonographie bis zu 3 weiteren Organen (Nieren, Hoden) & 4,66 & 10,73 \\
\hline & Labor & 1 fach $€$ & 1,15 fach $€$ \\
\hline 3908.H3* & PSA-Bestimmung & 17,49 & 20,11 \\
\hline
\end{tabular}

Früherkennung Blasenkrebs

\begin{tabular}{|l|l|r|r|}
\hline GOÄ Nr. & Kurzlegende & $\mathbf{1 f a c h} €$ & $\mathbf{2 , 3 f a c h} €$ \\
\hline $\mathbf{3}$ & $\begin{array}{l}\text { Eingehende Beratung (mind. 10 Min.) - nicht neben Sonderleis- } \\
\text { tungen }\end{array}$ & 8,74 & $\mathbf{2 0 , 1 1}$ \\
\hline $\mathbf{4 1 0}$ & Sonographie, ein Organ (Blase) & 11,66 & $\mathbf{2 6 , 8 1}$ \\
\hline $\mathbf{4 0 3}{ }^{\star}$ & Zuschlag zur Sonographie bei transrectaler Untersuchung & 8,74 & $\mathbf{1 5 , 7 4}$ \\
\hline
\end{tabular}


E. Individuelle Gesundheitsleistungen von A - Z

\section{Bestimmung: Tumormarker NMP22 (Nuclear matrix protein 22)}

Der Blasentumor ist eine relativ häufige Krebserkrankung und steht nach dem Prostatakrebs mit ca. $34 \%$ an zweiter Stelle der urologischen Tumore. Männer sind häufiger betroffen als Frauen.

Der Tumormarker NMP22 wurde in den USA entwickelt und von der FDA (U.S. Food and Drug Administration) - neben der Therapieüberwachung - auch für die Früherkennung (z. B. Sreening von Personen mit hohem Risiko für die Entwicklung eines Harnblasenkarzinoms: Raucher, Arbeiter in chemischen Betrieben) zugelassen.

Andrologische Diagnostik ohne Hinweis auf Vorliegen einer Sterilität oder nach Sterilisation

\begin{tabular}{|l|l|r|r|}
\hline GOÄ Nr. & Kurzlegende & $\mathbf{1 f a c h ~} €$ & $\mathbf{2 , 3 f a c h} €$ \\
\hline $\mathbf{3}$ & Eingeh. Beratung (mind. 10 Min.) - nicht neben Sonderleistungen & $\mathbf{8 , 7 4}$ & $\mathbf{2 0 , 1 1}$ \\
\hline $\mathbf{6}$ & Nieren, harnableitende Wege und männliche Genitalorgane & 5,83 & $\mathbf{1 3 , 4 1}$ \\
\hline & \multicolumn{1}{|c|}{ Labor } & $\mathbf{1 f a c h} €$ & $\mathbf{1 , 1 5 f a c h} €$ \\
\hline $\mathbf{3 6 6 7}^{*}$ & Spermienzahl, Motilitätsbeurteilung, mikroskopisch & 4,08 & $\mathbf{4 , 6 9}$ \\
\hline $\mathbf{3 6 6 8}^{*}$ & Spermiogramm & 23,32 & $\mathbf{2 6 , 8 1}$ \\
\hline
\end{tabular}

\section{Erektile Dysfunktion}

\begin{tabular}{|c|c|c|c|}
\hline GOÄ Nr. & Kurzlegende & 1 fach $€$ & $\begin{array}{r}{ }^{*} 1,8 / \\
2,3 f a c h €\end{array}$ \\
\hline 3 & Eingeh. Beratung (mind. 10 Min.) - nicht neben Sonderleistungen & 8,74 & 20,11 \\
\hline \begin{tabular}{|l|}
$643^{*}$ \\
analog
\end{tabular} & $\begin{array}{l}\text { Nicht direktionale Untersuchungen der Penisgefäße u./o. } \\
\text { Skrotalfächer - analog GOÄ Nr. 643 - entspr. GOÄ § } 6(2)^{11} \\
\text { GOÄ Kurztext Nr. 643: Periphere Arterien- bzw. Venendruck-und/oder Strömungs- } \\
\text { messung }\end{array}$ & 7,00 & 12,59 \\
\hline 1754 & $\begin{array}{l}\text { Direktionale Doppler-Sonographie der Penisgefäße u./o. } \\
\text { Skrotalfächer }\end{array}$ & 10,49 & 24,13 \\
\hline \multirow[t]{2}{*}{$250^{*}$} & Blutentnahme venös & 2,33 & 4,20 \\
\hline & Labor & 1 fach $€$ & 1,15 fach $€$ \\
\hline 4042 & Testosteron* & 20,40 & 23,46 \\
\hline
\end{tabular}

1) Bei analogen Leistungen muss - mind. in Kurzform - auch die Original-Leistungslegende angegeben werden.

Sexualberatung

\begin{tabular}{|c|c|c|c|}
\hline GOÄ Nr. & Kurzlegende & 1 fach $€$ & 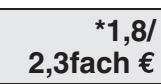 \\
\hline $\begin{array}{l}31 \\
\text { analog }\end{array}$ & $\begin{array}{l}\text { Ausführliche Sexualanamnese (30 Minuten), n. n. Nr. } 34 \text { - } \\
\text { analog GOÄ Nr. } 31 \text { - entspr. GOÄ } \S 6(2)^{1)}\end{array}$ & 26,23 & 60,33 \\
\hline 7 & Organsystemuntersuchung & 9,33 & 21,45 \\
\hline 34 & Beratung Lebensveränderung, n. n. Nr. A 31 & 8,74 & 20,11 \\
\hline \multirow[t]{2}{*}{$250^{*}$} & Blutentnahme venös & 2,33 & 4,20 \\
\hline & Labor & 1 fach $€$ & 1,15 fach $€$ \\
\hline $3908 \mathrm{H} 3$ & PSA & 17,49 & 20,11 \\
\hline 3765 & Sexualhormonbindendes Globulin* & 26,23 & 30,16 \\
\hline 4021 & $\mathrm{FSH}^{*}$ & 14,57 & 16,76 \\
\hline 4039 & Ötradiol $^{*}$ & 20,40 & 23,46 \\
\hline 4042 & Testosteron* & 20,40 & 23,46 \\
\hline
\end{tabular}

1) Bei analogen Leistungen muss - mind. in Kurzform - auch die Original-Leistungslegende angegeben werden.

* Untersuchungen aus Laborkapitel M III kann nur der Arzt abrechnen, der diese Leistungen selber erbracht hat. 
Sterilisation des Mannes

\begin{tabular}{|l|l|r|r|}
\hline GOÄ Nr. & Kurzlegende & $\mathbf{1}$ fach $€$ & $\mathbf{2 , 3 f a c h} €$ \\
\hline $\mathbf{3}$ & $\begin{array}{l}\text { Eingehende Beratung (mind. 10 Min.) - nicht neben Sonderleis- } \\
\text { tungen }\end{array}$ & 8,74 & $\mathbf{2 0 , 1 1}$ \\
\hline $\mathbf{6}$ & Nieren, harnableitende Wege und männliche Genitalorgane & 5,83 & $\mathbf{1 3 , 4 1}$ \\
\hline $\mathbf{1 7 5 6}$ & Unterbindung beider Samenleiter - auch mit Teilresktion & 48,50 & $\mathbf{1 1 1 , 5 4}$ \\
\hline
\end{tabular}

Zusätzlich ggf. praeoperative Diagnostik, Anästhesieleistungen und nach Sterilisation Kontrolluntersuchung (Andrologische Diagnostik mit Spermiogramm)

Vorhautbeschneidung ohne medizinische Indikation

\begin{tabular}{|c|c|c|c|}
\hline GOÄ Nr. & Kurzlegende & 1 fach $€$ & 2,3 fach $€$ \\
\hline 3 & $\begin{array}{l}\text { Eingehende Beratung (mind. } 10 \text { Min.) - nicht neben Sonderleis- } \\
\text { tungen }\end{array}$ & 8,74 & 20,11 \\
\hline 5 & Symptombezogene Untersuchung & 4,66 & 10,73 \\
\hline 1741 & Vorhautbeschneidung ohne medizinisc & 21,57 & 49,60 \\
\hline 442 & $\begin{array}{l}\text { Zuschlag bei amb. Durchführung der Leistung nach Nr. } 1741 \text { - } \\
\text { nur } 1 \text { fach berechenbar }\end{array}$ & 23,32 & - \\
\hline
\end{tabular}

